\title{
A general method to improve fluorophores using deuterated auxochromes
}

\author{
Jonathan B. Grimm ${ }^{1}$, Liangqi Xie ${ }^{1}$, Jason C. Casler ${ }^{2}$, Ronak Patel ${ }^{1}$, Ariana N. Tkachuk ${ }^{1}$, Natalie Falco ${ }^{1}$, Heejun \\ Choi $^{1}$, Jennifer Lippincott-Schwartz ${ }^{1}$, Timothy A. Brown ${ }^{1}$, Benjamin S. Glick ${ }^{2}$, Zhe Liu ${ }^{1}$, and Luke D. Lavis ${ }^{1 * *}$ \\ ${ }^{1}$ Janelia Research Campus, The Howard Hughes Medical Institute, 19700 Helix Drive, Ashburn, VA 20147, USA \\ ${ }^{2}$ Department of Molecular Genetics and Cell Biology, University of Chicago, 920 East $58^{\text {th }}$ Street, Chicago, IL \\ 60637, USA
}

*Email: lavis1@janelia.hhmi.org

EXPERIMENTAL INFORMATION

Page Contents

S2 Figures S1-S6

S7 Schemes S1-S3

S10 Experimental Details for Spectroscopy and Imaging

S15 Experimental Details and Characterization Data for All Compounds

S34 References

S35 NMR and HPLC-MS 


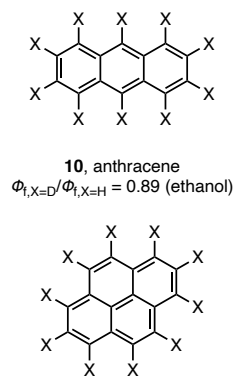

11 , pyrene
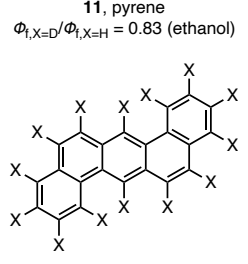

12, benzo $[k]$ tetraphene
$\Phi_{\mathrm{t}, \mathrm{X}=\mathrm{D}} / \Phi_{\mathrm{t}, \mathrm{X}=\mathrm{H}}=0.87$ (ethanol)
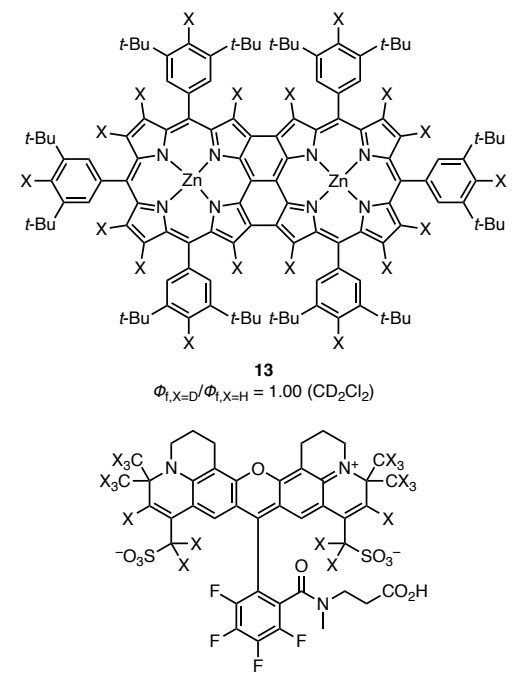

$\Phi_{\mathrm{f}, \mathrm{X}=\mathrm{D}} / \Phi_{\mathrm{t}, \mathrm{X}=\mathrm{H}}=0.98\left(\mathrm{H}_{2} \mathrm{O}\right)$

Figure S1. Chemical structures and fluorescence quantum yields $\left(\Phi_{\mathrm{f}}\right)$ of matched pairs of nondeuterated $(X=H)$ and deuterated $(X=D)$ fluorophores, including polycyclic aromatic compounds (10-12), a porphyrin (13), and a rhodamine (14). 
a
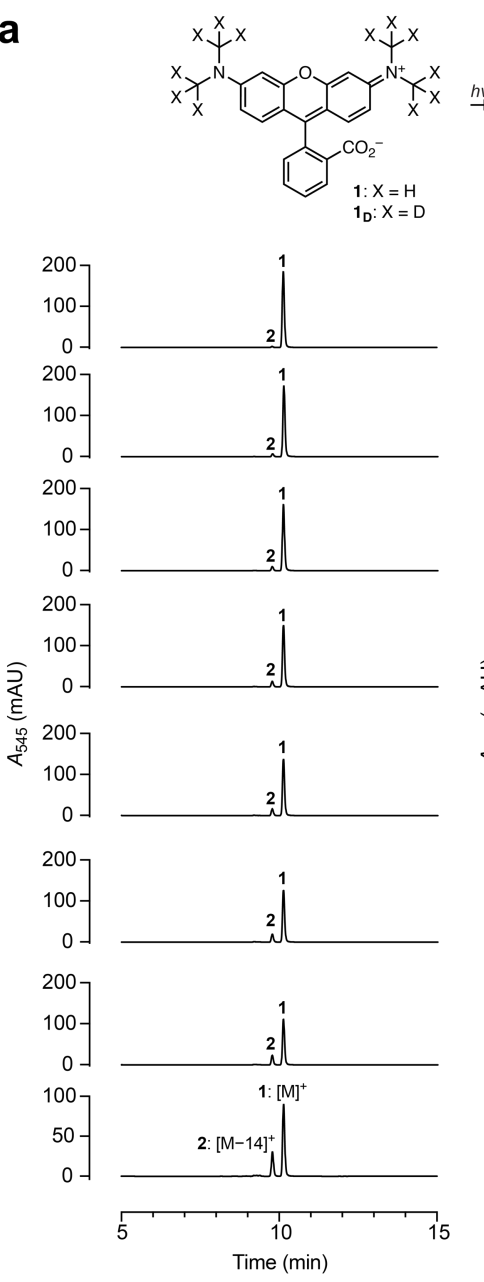
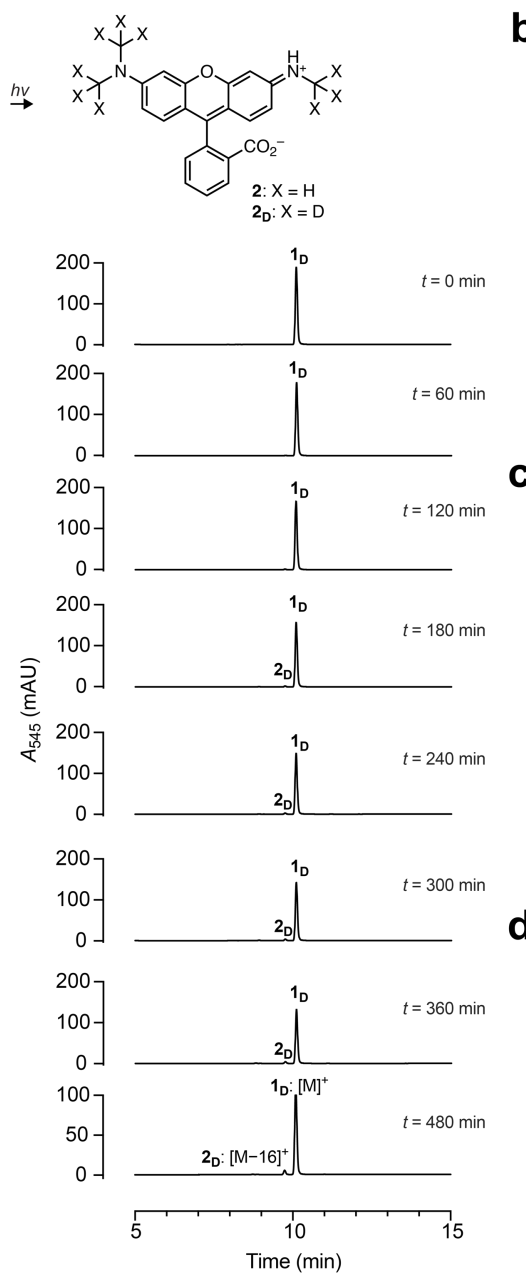

b

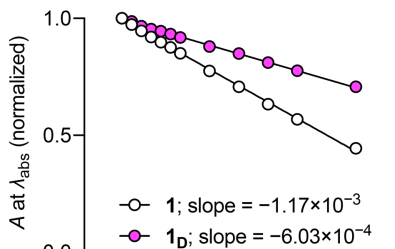

-o- $1_{D} ;$ slope $=-6.03 \times 10^{-4}$
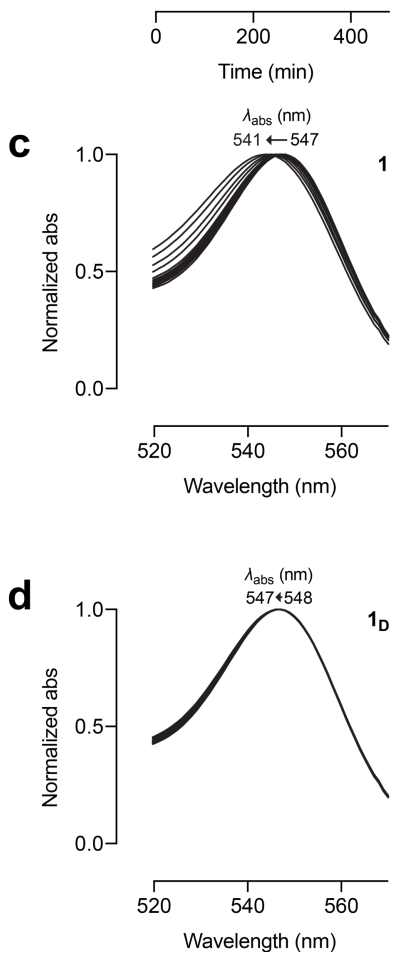

Figure S2. Photobleaching of TMR (1) and TMR- $d_{12}$ (1D). (a) Photoinduced dealkylation of 1 or $1_{D}$ to form 2 or 2D, respectively, and reaction monitoring by tandem liquid chromatography-mass spectrometry (LC-MS) confirming loss of methyl groups. (b) Relative absorption vs. time and initial slopes of 1 at $\lambda_{\text {abs }}=547 \mathrm{~nm}$ or $1 \mathrm{D}$ at $\lambda_{\mathrm{abs}}=548 \mathrm{~nm}$ during photobleaching $\left(560 \mathrm{~nm} ; 1.02 \mathrm{~W} / \mathrm{cm}^{2}\right)$; full spectra are given in Figure 2e,f. (c,d) Independently normalized absorption spectra over time for 1 (c) or $\mathbf{1}_{\mathrm{D}}$ (d) during photobleaching $\left(560 \mathrm{~nm} ; 1.02 \mathrm{~W} / \mathrm{cm}^{2}\right)$ to show improvement in chromostability upon deuteration of the $\mathrm{N}$-methyl groups in $\mathbf{1}_{\mathrm{D}}$. 
a
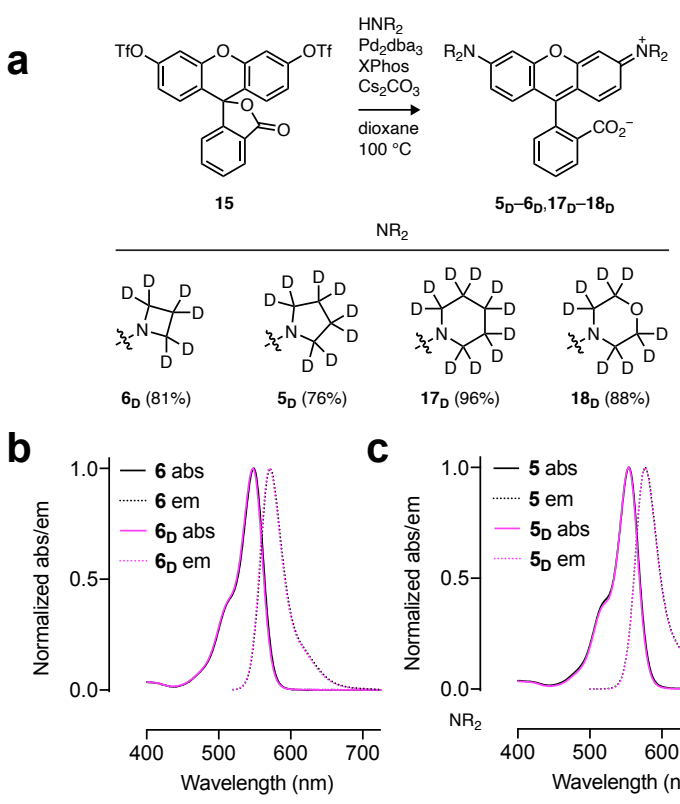

C
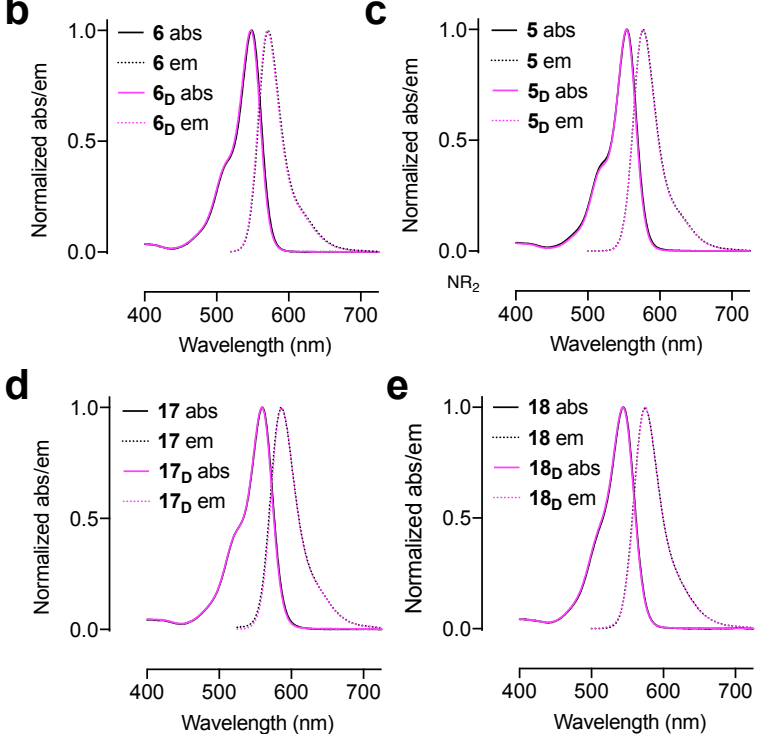

Figure S3. (a) Synthesis $5_{D}-6_{D}$ and $17_{D}-18_{D}$ from fluorescein ditriflate (15). (b-e) Normalized absorption (abs) and fluorescence emission (em) spectra for matched pairs of nondeuterated and deuterated rhodamine dyes: (b) $\mathbf{6}$ and $\mathbf{6}_{\mathrm{D}}$; (c) 5 and $5_{\mathrm{D}}$; (d) 17 and 17D; (e) 18 and 18D. 
a
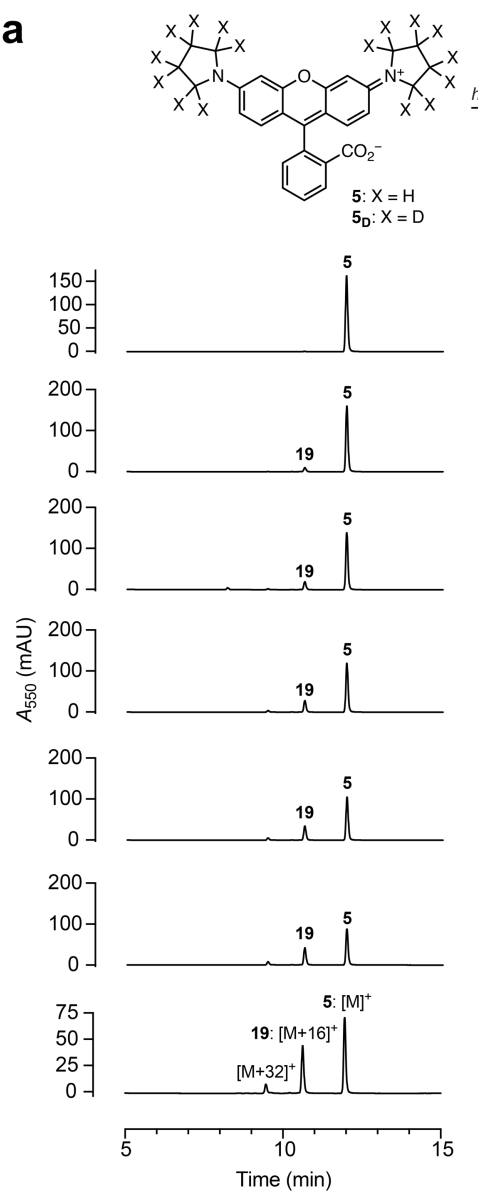
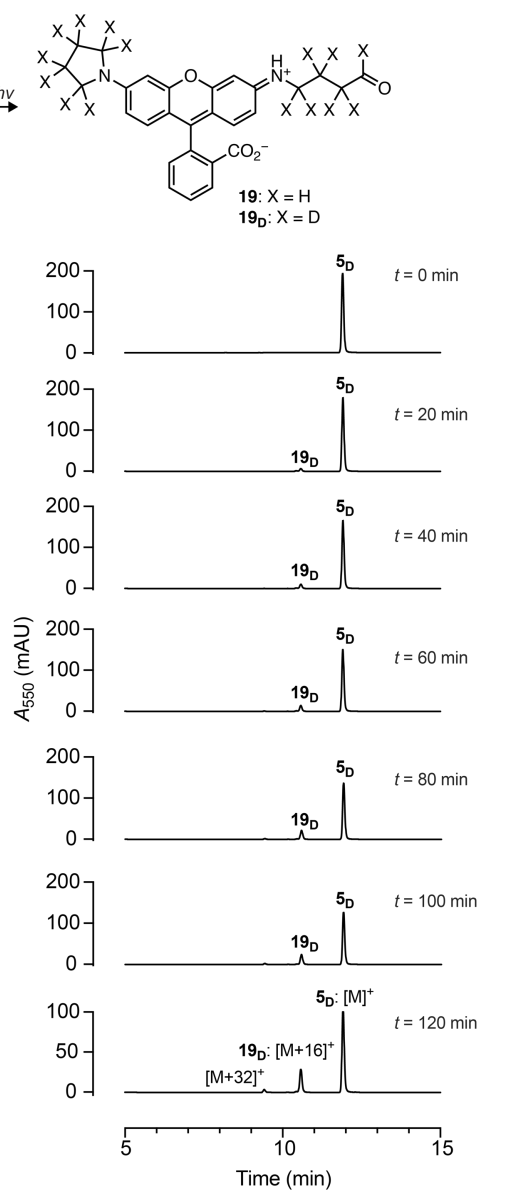

b

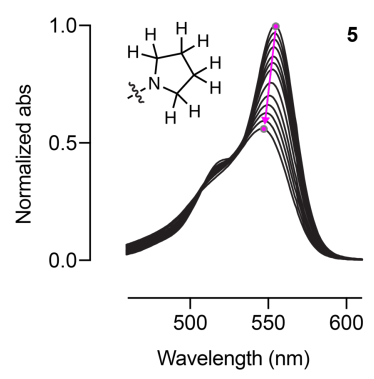

C

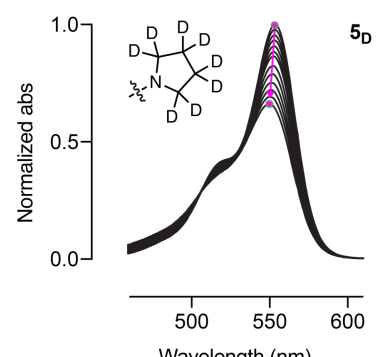

Wavelength (nm)

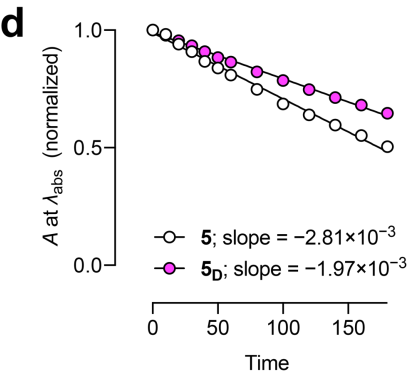

Figure S4. Photobleaching of 5 and $5_{D}$. (a) Photoinduced dealkylation of 5 or $5_{D}$ to form 19 or $19_{D}$, respectively, and reaction monitoring by tandem liquid chromatography-mass spectrometry (LC-MS) confirming addition of oxygen. (b,c) Sequential absorption spectra of 5 (b) and $\mathbf{5}_{\mathrm{D}}$ (c) during photobleaching at $560 \mathrm{~nm}\left(1.02 \mathrm{~W} / \mathrm{cm}^{2}\right)$. The magenta arrows highlight the shift in $\lambda_{\text {abs }}$ and intensity over time. (d) Relative absorption vs. time and initial slopes of 5 at $\lambda_{\text {abs }}=553 \mathrm{~nm}$ or $5_{\mathrm{D}}$ at $\lambda_{\mathrm{abs}}=554 \mathrm{~nm}$ during photobleaching $\left(560 \mathrm{~nm} ; 1.02 \mathrm{~W} / \mathrm{cm}^{2}\right)$; full spectra are given in $(\mathbf{b}, \mathbf{c})$. 
a

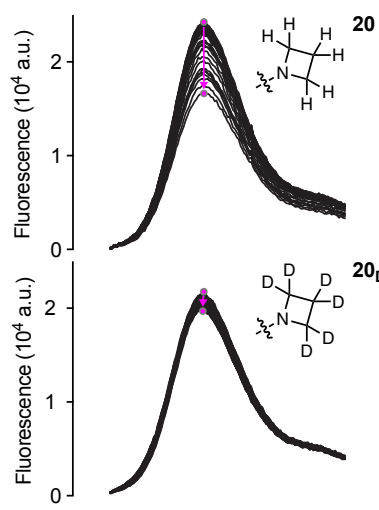

$540 \quad 560580600620$ Wavelength $(\mathrm{nm})$

e

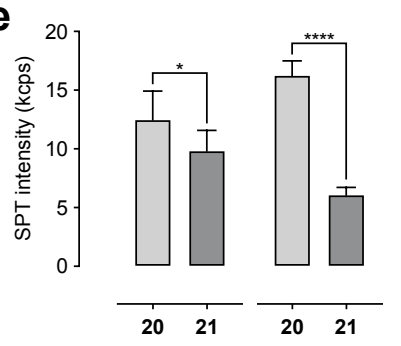

b
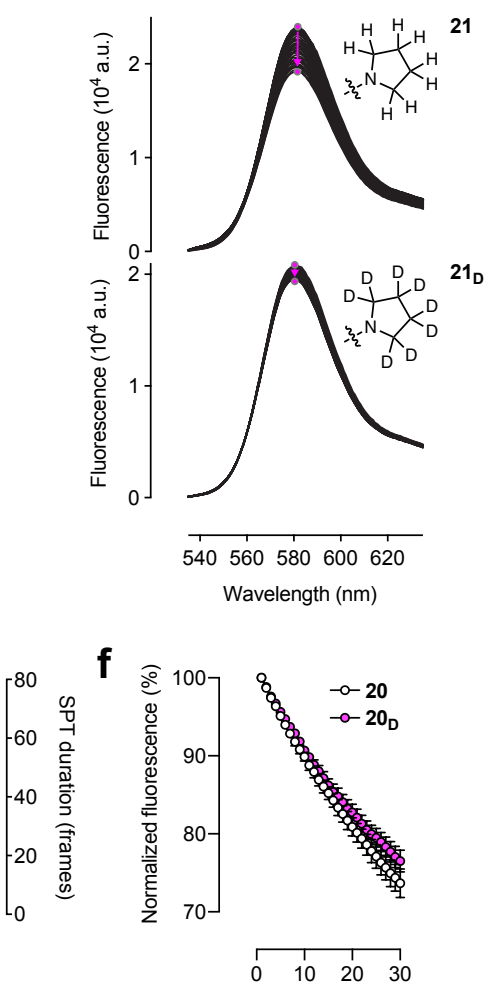

c

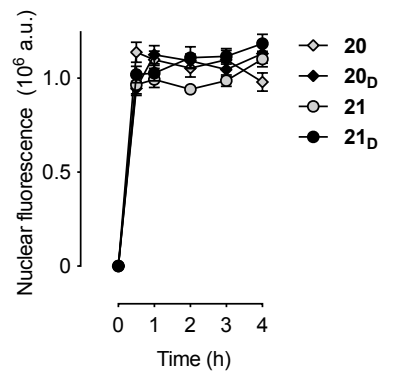

d $\left.\begin{array}{r}4.4 \\ 4.3- \\ 4.2- \\ 5.5 \\ 4.1- \\ 4.0- \\ 3.9\end{array}\right]$
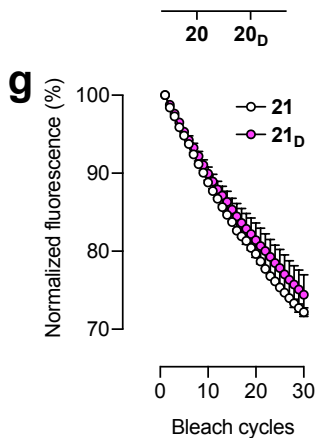

Figure S5. (a,b) Sequential fluorescence emission spectra of (a) 20:HaloTag protein conjugate and 20 D $_{\mathrm{D}}$ HaloTag protein conjugate or (b) 21:HaloTag protein conjugate and 21 :HaloTag protein conjugate during illumination; the magenta arrows show the shift in $\lambda_{\mathrm{em}}$ over time. (c) Nuclear fluorescence vs. time upon addition of HaloTag ligands 20, 20D, 21, and 21D (200 nM) to live U2OS cells expressing HaloTag-histone H2B; error bars indicate SEM. (d) Plot of fluorescence lifetime $(\tau)$ of U2OS cells expressing HaloTag-histone H2B and labeled with HaloTag ligands 20 or 20D; center line indicates median; box limits indicate upper and lower quartiles; whiskers indicate min-max. (e) Plot of average single-molecule intensity and trajectory length per cell in single-particle tracking experiments using $\mathrm{JF}_{549}$-HaloTag ligand (20) or pyrrolidine-containing HaloTag ligand 21; error bars indicate SEM. (f,g) Nuclear fluorescence during photobleaching in fixed U2OS cells expressing HaloTag-histone H2B and labeled with 20 and 20D (f) or with 21 and 21D (g). 

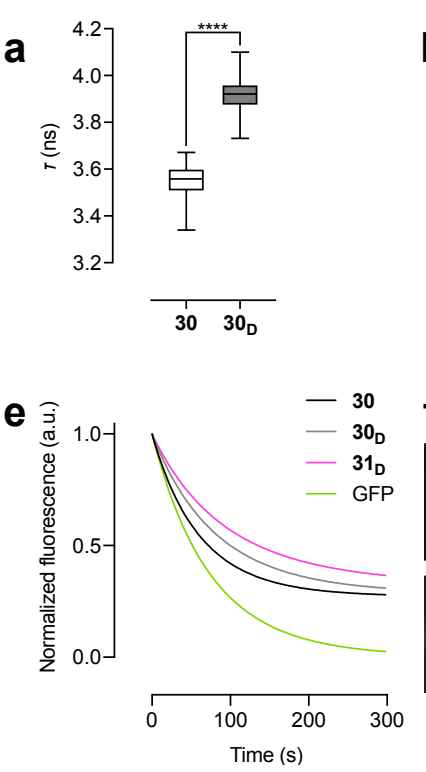
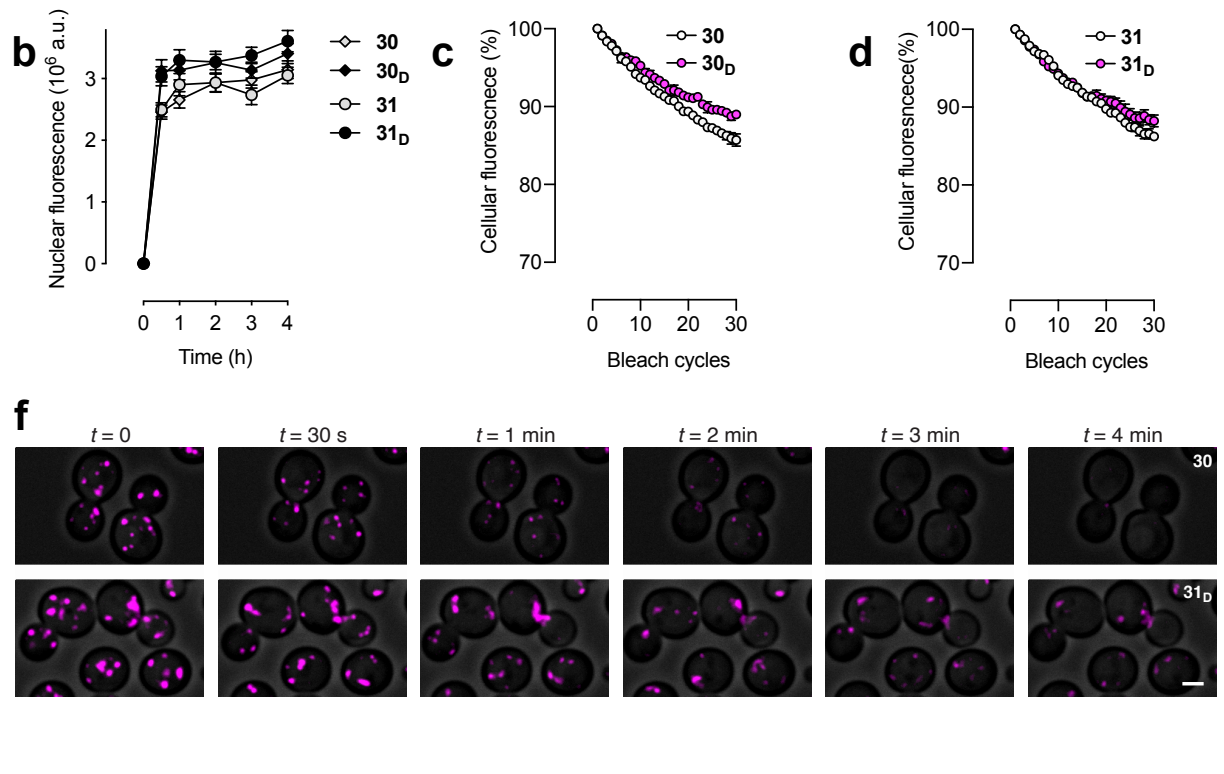

Figure S6. (a) Plot of fluorescence lifetime $(T)$ of U2OS cells expressing HaloTag-histone H2B and labeled with HaloTag ligands 30 or 30D; center line indicates median; box limits indicate upper and lower quartiles; whiskers indicate min-max. (b) Nuclear fluorescence vs. time upon addition of ligands 30, 30, 31 , or $31_{D}(200 \mathrm{nM})$ to live U2OS cells expressing HaloTag-histone H2B; error bars indicate SEM. (c,d) Cellular fluorescence during photobleaching in fixed cells expressing HaloTag-histone H2B and labeled with (c) 30 and 30 or with (d) 31 and 31 D. (e) Photobleaching plot of normalized fluorescence intensity vs. time of $S$. cerevisiae cells expressing Sec7-iGFP-HaloTag fusions and labeled with ligands 30, 30D, or 31 D. (f) Expanded image montage from Figure 5g/Movie S1 showing S. cerevisiae labeled with 30 or $31_{\mathrm{D}}$; scale bar: $2 \mu \mathrm{m}$. 
Scheme S1. Synthesis of HaloTag ligands 20D, 21, and 21D.

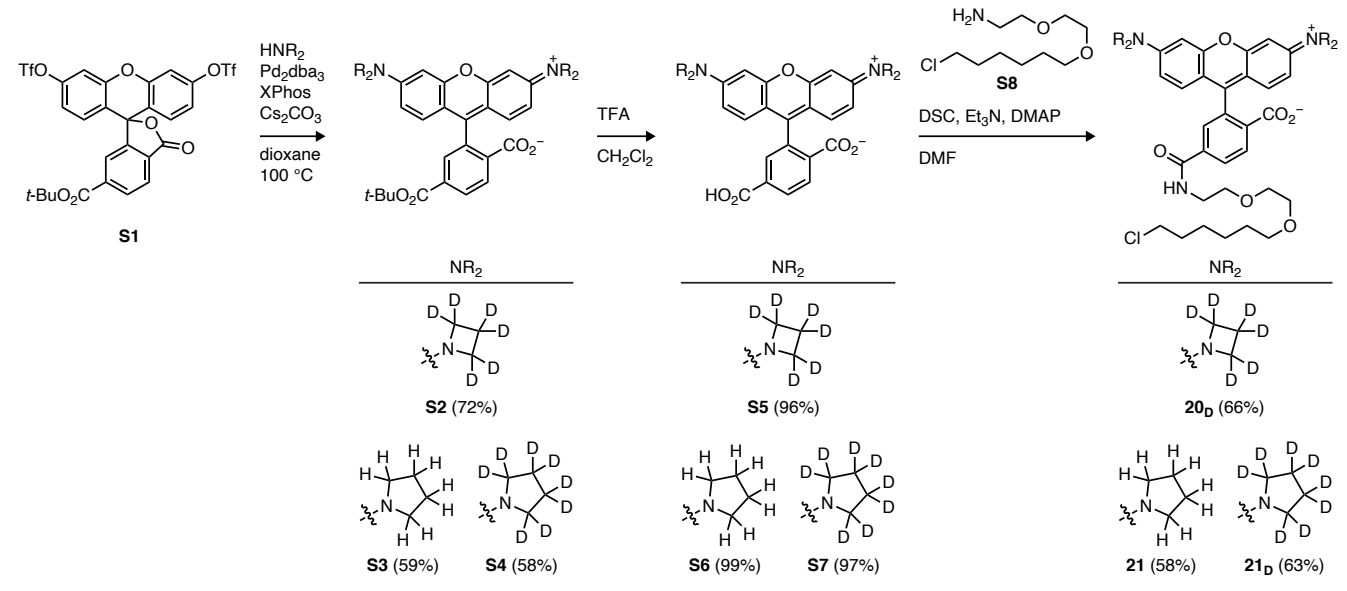

Scheme S2. Synthesis of nondeuterated and deuterated dyes: (a) coumarins, (b) phenoxazines, (c) fluorinated rhodamines, (d) carborhodamines, and (e) Si-rhodamines.

a<smiles>Cc1cc(=O)oc2cc(O)ccc12</smiles>

s9
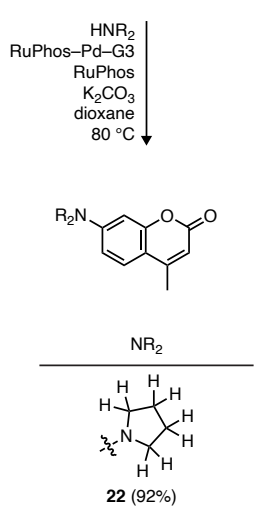

$\underbrace{D}_{2=} \underbrace{D}_{D}$

22 $\mathrm{D}(86 \%)$
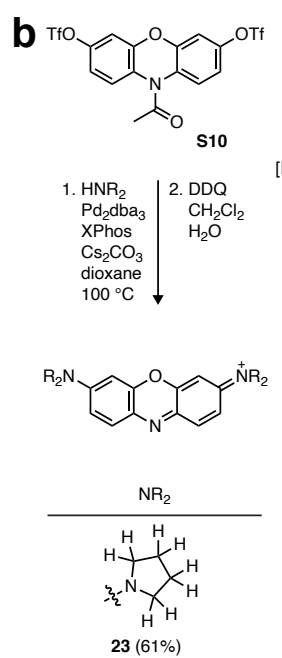

$\underbrace{D}_{-2} \underbrace{D}_{D}$

23 $\mathrm{D}(34 \%)$

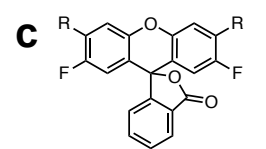

$\left[\mathrm{RuCp}^{*}(\mathrm{MeCN})_{3}\right] \mathrm{OTf}-\mathrm{S} 11: \mathrm{R}=\mathrm{OTf}$

$\mathrm{RuCp}^{*}(\mathrm{MeCN})_{3} \mathrm{OTf}$
$\mathrm{Bu}_{4} \mathrm{NI}, \mathrm{DMI}, 100^{\circ} \mathrm{C}$

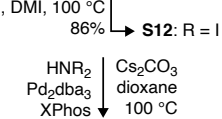

$X$ Phos $\downarrow 100^{\circ} \mathrm{C}$
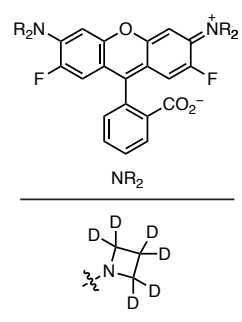

$24 \mathrm{D}(66 \%)$

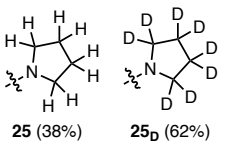

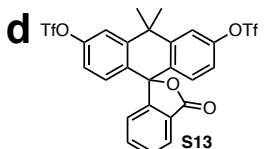
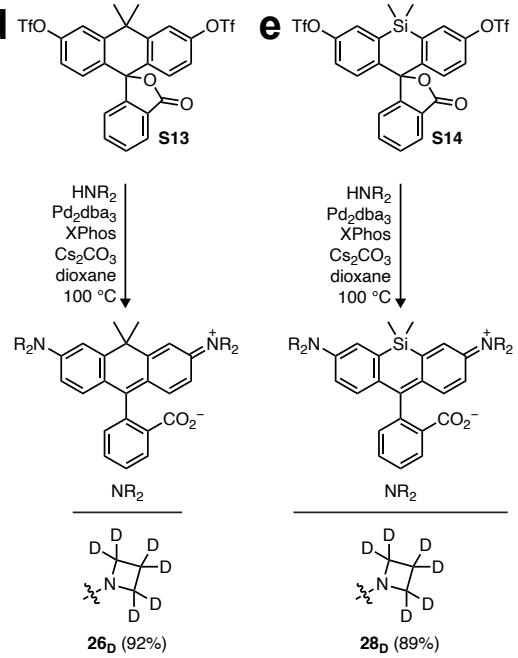

$\underbrace{D}_{-\frac{2}{N}} \underbrace{D}_{D} \underbrace{D}_{D}$

27 D $(91 \%)$

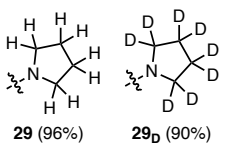


Scheme S3. Synthesis of HaloTag ligands $30_{D}, 31$, and 31 .
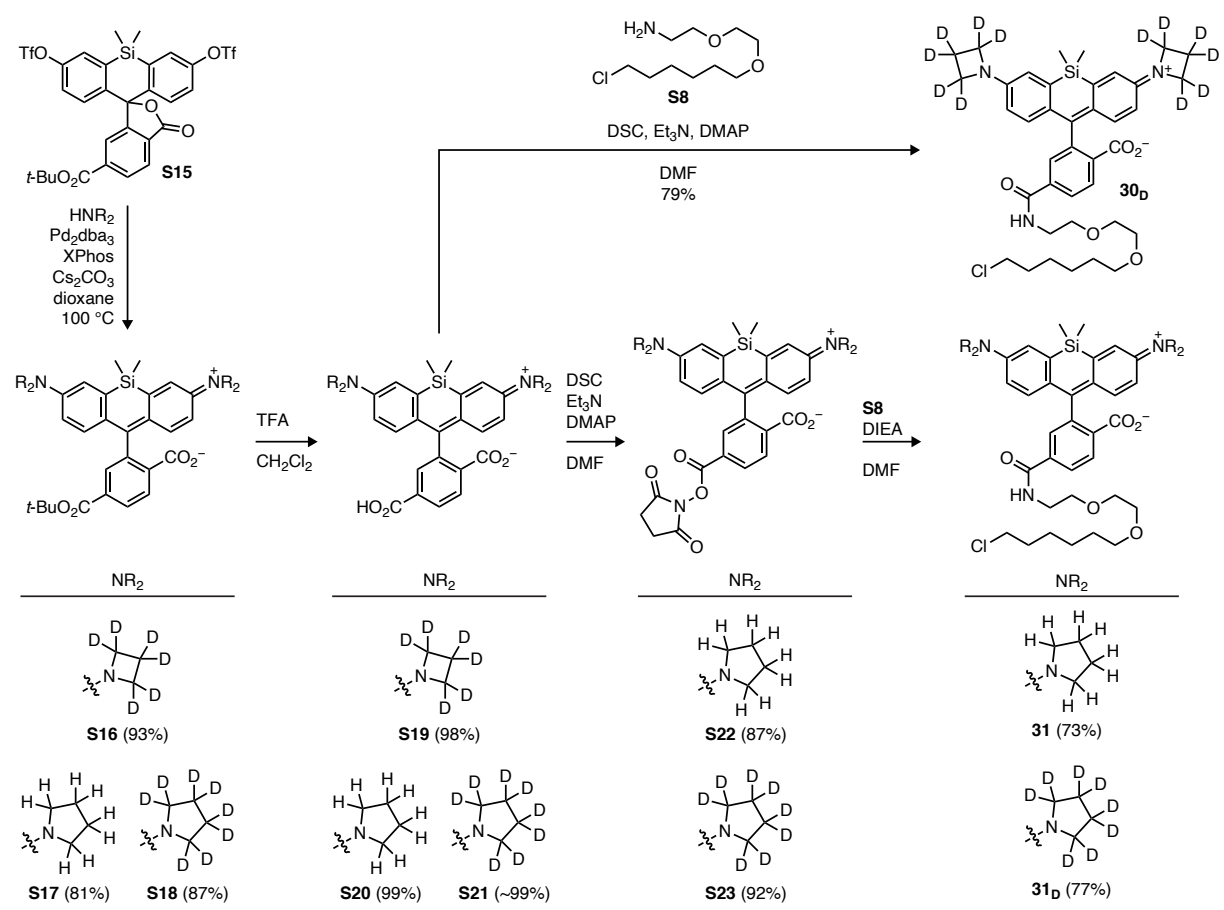

管

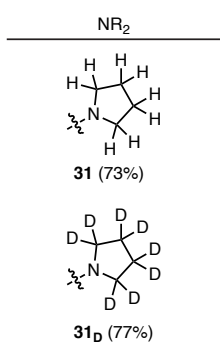




\section{GENERAL EXPERIMENTAL INFORMATION FOR SPECTROSCOPY AND IMAGING}

Compound sources. Tetramethylrhodamine (1; Reference Standard Grade) was purchased from AAT BioQuest. Compounds $5,6,17,18,20,24,26-28$, and 30 were available from previous work. ${ }^{1-4}$

UV-vis and fluorescence spectroscopy (Figure 2b, Table 1, Table 2, Table 3, Figure S3b-e). Fluorescent molecules for spectroscopy were prepared as stock solutions in DMSO and diluted such that the DMSO concentration did not exceed $1 \% \mathrm{v} / \mathrm{v}$. Spectroscopy was performed using 1-cm path length, $3.5-\mathrm{mL}$ quartz cuvettes or $1-\mathrm{cm}$ path length, $1.0-\mathrm{mL}$ quartz microcuvettes from Starna Cells. All measurements were taken at ambient temperature $(22 \pm 2$ $\left.{ }^{\circ} \mathrm{C}\right)$. Absorption spectra were recorded on a Cary Model 100 spectrometer (Agilent). Fluorescence spectra were recorded on a Cary Eclipse fluorometer (Varian). Maximum absorption wavelength $\left(\lambda_{\text {abs }}\right)$, extinction coefficient $(\varepsilon)$, and maximum emission wavelength $\left(\lambda_{\mathrm{em}}\right)$ were measured in $10 \mathrm{mM}$ HEPES, $\mathrm{pH} 7.3$ buffer. Normalized spectra are shown for clarity.

Quantum yield determination (Table 1, Table 2, Table 3). All reported absolute fluorescence quantum yield values $(\Phi)$ were measured in our laboratory under identical conditions using a Quantaurus-QY spectrometer (model C11374, Hamamatsu). This instrument uses an integrating sphere to determine photons absorbed and emitted by a sample. Measurements were carried out using dilute samples $(A<0.1)$ and self-absorption corrections ${ }^{5}$ were performed using the instrument software. Reported values are averages $(n=3)$.

Determination of $K_{\mathrm{L}-\mathrm{Z}}$ (Table 1, Table 3). We calculated $K_{\mathrm{L}-\mathrm{Z}}$ using the following equation ${ }^{6}: K_{\mathrm{L}-\mathrm{Z}}=\left(\varepsilon_{\mathrm{dw}} / \varepsilon_{\max }\right) /(1-$ $\left.\varepsilon_{\mathrm{dw}} / \varepsilon_{\max }\right) . \varepsilon_{\mathrm{dw}}$ is the extinction coefficient of the dyes in a $1: 1 \mathrm{v} / \mathrm{v}$ dioxane:water solvent mixture; this dioxane-water mixture was chosen to give the maximum spread of $K_{\mathrm{L}-Z}$ values ${ }^{7}$. $\varepsilon_{\max }$ refers to the maximal extinction coefficients measured in different solvent mixtures empirically determined depending on dye type: $0.1 \% \mathrm{v} / \mathrm{v}$ TFA in ethanol for the Si-rhodamines 28 and 28 $\mathbf{2} ; 0.1 \% \mathrm{v} / \mathrm{v}$ trifluoroacetic acid (TFA) in 2,2,2-trifluoroethanol (TFE) for all the other rhodamine variants.

Photostability and chromostability measurements of dyes by absorption spectroscopy and LC-MS (Figure 2cf, Figure 3b,c, Figure S2, Figure S4). To test the stability of the free dyes, solutions of $\mathbf{1}, \mathbf{1}_{\mathbf{D}}, \mathbf{5}$, and $\mathbf{5} \mathbf{D}(10 \mu \mathrm{M})$ were prepared in $10 \mathrm{mM}$ HEPES, $\mathrm{pH}$ 7.3. For each dye, two $3 \mathrm{~mL}$ samples were prepared in 1-cm path length, 3.5-mL quartz cuvettes (Starna Cells). Samples were placed under a Mightex super high-powered LED collimator source (560 $\mathrm{nm}$ broadband, $110 \mathrm{~nm}$ FWHM, $22 \mathrm{~mm}$ aperture, $3500 \mathrm{~mW}$ typical output power), which was vertically mounted 7 $\mathrm{cm}$ above a mirrored disc $(\mathrm{d}=2 \mathrm{~cm})$ on a black acrylic base. The LED was driven by a ThorLabs DC2200 high-power 1-channel LED driver (10 A, 100\% LED brightness, pulse mode, $1000 \mathrm{~ms}$ on-time, $0.001 \mathrm{~ms}$ off-time). Samples were bleached for the indicated amount of time with a measured irradiance of $1.02 \mathrm{~W} / \mathrm{cm}^{2}$ (ThorLabs PM100A), pausing at regular time intervals (10-20 min) to record the absorption spectrum on a Cary Model 100 spectrometer and remove a small aliquot for LC-MS analysis (Agilent Eclipse XDB 4.6 × 150 mm $5 \mu \mathrm{m}$ C18 column; $20 \mu \mathrm{L}$ injection; 10-95\% 
$\mathrm{MeCN} / \mathrm{H}_{2} \mathrm{O}$, linear gradient, with constant $0.1 \% \mathrm{v} / \mathrm{v}$ TFA additive; $20 \mathrm{~min}$ run; $1 \mathrm{~mL} / \mathrm{min}$ flow; ESI; positive ion mode; detection at 545 or $550 \mathrm{~nm}$ ).

Photostability and chromostability measurements of dyes and HaloTag conjugates by fluorescence emission (Figure 3d,e, Figure S5a,b). To test the stability of the free dyes, solutions of 5, 5D, 6, and $\mathbf{6}_{\mathrm{D}}(1 \mu \mathrm{M})$ were prepared in $10 \mathrm{mM}$ HEPES, $\mathrm{pH}$ 7.3. To test the stability of the HaloTag conjugates, HaloTag protein was used as a $200 \mu \mathrm{M}$ solution in PBS, pH 7.4. Solutions of HaloTag ligands 20, 20D, 21, and 21 $\mathbf{D}(5 \mu \mathrm{M})$ were prepared in $10 \mathrm{mM}$ HEPES, pH 7.3 containing $0.1 \mathrm{mg} \cdot \mathrm{mL}^{-1}$ CHAPS. An aliquot of HaloTag protein (2.0 equiv, $10 \mu \mathrm{M}$ final [HaloTag]) was added to each sample, and the resulting mixture was incubated at $4{ }^{\circ} \mathrm{C}$ overnight. Stability measurements were performed after the HaloTag conjugate solutions were diluted $5 \times(1 \mu \mathrm{M}$ final [ligand] $)$ into $10 \mathrm{mM}$ HEPES, pH 7.3 buffer solution. An aliquot of these solutions was added to each well of 10 -well Teflon printed glass slide with $1.5 \mathrm{~mm}$ well diameter (Tekdon, Inc.) and sealed with a coverslip using vacuum grease. The hydrophobic coating of the slide resulted in formation of macrodroplets of aqueous dye solution in each well. Fluorophore bleaching was measured by illuminating an entire droplet using an upright microscope (Zeiss Axio Observer Z2) and a 5×/0.25 NA objective. The fluorophore and HaloTag conjugate solutions were bleached using an upright microscope (Zeiss Axio Observer Z2) $5 \times / 0.25$ NA objective. Light illumination was provided by a $532 \mathrm{~nm}$ laser (Compass 215M-75; Coherent) at $18 \mathrm{~mW}$ power (intensity $0.96 \mathrm{~W} / \mathrm{cm}^{2}$ ). Fluorescence emission was collected using a 590/104 filter and emission spectra were measured using a fiber coupled spectrometer (QE 65000; Ocean Optics). Each sample was continuously bleached for $40 \mathrm{~min}$ with fluorescence spectra acquired every $1 \mathrm{~min}$. Spectra are averages $(n=2)$.

Quantum yield determination of HaloTag protein conjugates (Figure 4b, Figure 5b). HaloTag protein was used as a $100 \mu \mathrm{M}$ solution in $75 \mathrm{mM} \mathrm{NaCl}, 50 \mathrm{mM}$ TRIS· $\mathrm{HCl}$, pH 7.4 with $50 \% \mathrm{v} / \mathrm{v}$ glycerol (TBS-glycerol). A solution of HaloTag ligand 20, 20D, 30, or 30 $(5 \mu \mathrm{M})$ was prepared in $10 \mathrm{mM}$ HEPES, $\mathrm{pH} 7.3$ containing $0.1 \mathrm{mg} \cdot \mathrm{mL}^{-1} \mathrm{CHAPS}$. An aliquot of HaloTag protein (1.5 equiv, $7.5 \mu \mathrm{M}$ final [HaloTag]) was added and the resulting mixture was incubated until consistent absorption signal was observed (60-120 min). After incubation, this solution was diluted 5-10-fold in $10 \mathrm{mM}$ HEPES, pH 7.3 containing $0.1 \mathrm{mg} \cdot \mathrm{mL}^{-1}$ CHAPS to give $500 \mathrm{nM}$ dye concentration, which is optimal for quantum yield determination $(n=14)$.

Confocal fluorescence microscopy (Figure 4c, Figure 5c). U2OS cells (ATCC) with an integrated histone H2BHaloTag fusion protein expressing plasmid via the piggyBac transposon system were cultured in Dulbecco's modified Eagle medium (DMEM, phenol red-free; Life Technologies) supplemented with $10 \% \mathrm{v} / \mathrm{v}$ fetal bovine serum (FBS, Life Technologies), $1 \mathrm{mM}$ GlutaMAX (Life Technologies) and maintained at $37{ }^{\circ} \mathrm{C}$ in a humidified $5 \%(\mathrm{v} / \mathrm{v}) \mathrm{CO}_{2}$ environment. These cell lines were kept under the selection of $500 \mu \mathrm{g} / \mathrm{mL}$ Geneticin (Life Technologies) and undergo regular mycoplasma testing by the Janelia Cell Culture Facility. Cells were incubated with HaloTag ligands 20, 20D, 21, 21D, 30, 30D, 31, or 31D (200 nM, 2 h) and washed 3× with dye-free media. Cells were imaged on a Zeiss LSM 800 confocal microscope with a Plan APO 63×/1.4 NA oil DIC M27 objective. Ligands 20, 20D, 21, and 21D were excited with a $561 \mathrm{~nm}$ laser and emission light was collected at 565-632 nm. Ligands 30, 30D, 31, and 31D were excited 
with a $640 \mathrm{~nm}$ laser, and emission light was collected at $656-700 \mathrm{~nm}$. The confocal images were processed using FIJI ${ }^{8}$ and displayed as maximum intensity image projections.

Dye loading kinetics (Figure S5c, Figure S6b). Live U2OS cells stably expressing histone H2B-HaloTag fusion proteins were labeled over a time course of $0-4 \mathrm{~h}$ with $200 \mathrm{nM}$ of HaloTag ligands 20, 20D, 21, 21D, 30, 30D, 31, or 31D. Cells were briefly washed $3 \times$ with dye-free media and immediately imaged live using widefield microscopy on a Nikon Eclipse Ti, Plan APO $\lambda 20 \times / 0.75$ air objective. Fluorescence was quantified from the average of the summed intensity of nuclear signals in single-plane widefield images analyzed using Nikon NIS-Elements AR software. Fields of view were chosen randomly; $n=100$ nuclear signals per compound.

In-cell fluorescence lifetime $(\tau)$ measurements (Figure S5d, Figure S6a). U2OS cells stably expressing histone H2B-HaloTag protein fusions were plated on a coverslip pre-coated with human fibronectin (Millipore). After $24 \mathrm{~h}$, the cells were incubated with $100 \mathrm{nM}$ of dyes 20, 20, 30, or 30, for 30 min in DMEM (Corning), supplemented with $10 \% \mathrm{v} / \mathrm{v}$ FBS (Corning), $2 \mathrm{mM}$ L-Glutamine, $100 \mathrm{U} / \mathrm{mL}$ penicillin and $100 \mu \mathrm{g} / \mathrm{mL}$ streptomycin at $37{ }^{\circ} \mathrm{C}$ and $5 \% \mathrm{CO}_{2}$. Prior to imaging the cells were briefly washed $3 \times$ with dye-free media. These cells were imaged using a Zeiss 880 laser scanning confocal microscope equipped with Ti:Sapphire laser (Coherent Chameleon). The labeled U2OS cell samples were excited with $850 \mathrm{~nm}\left(\mathbf{2 0}\right.$ and $\mathbf{2 0}$ D) or $830 \mathrm{~nm}\left(\mathbf{3 0}\right.$ and $\left.\mathbf{3 0}_{\mathbf{D}}\right)$. The excitation laser was filtered through a short-pass filter (675LP). The emitted light passed through a dichroic (DIC560) and collected by the PMA hybrid detector (PicoQuant) with bandpass filters (BPF600/50 for 20 and 20D; no filter for 30 and 30D) and fed to the singlephoton counting board (TimeHarp 260, PicoQuant) and monitored at $80 \mathrm{MHz}$. The resulting histogram was fit to a single exponential decay function using SymPhoTime 64 (PicoQuant); $n=30$ for $\mathbf{2 0}$ and $\mathbf{2 0}$ D, $n=27$ for 30, and $n=$ 29 for 30D.

Initial comparison of HaloTag ligands 20 and 21 in single-particle tracking (SPT) experiments (Figure S5e). U2OS cells stably expressing histone H2B-HaloTag protein fusions were plated on a coverslip pre-coated with human fibronectin (Millipore). After $24 \mathrm{~h}$, the cells were incubated with $1 \mathrm{nM}$ of either $\mathbf{2 0}$ or $\mathbf{2 1}$ for $30 \mathrm{~min}$ in (?) DMEM (Corning) supplemented with 10\% v/v FBS (Corning), $2 \mathrm{mM} \mathrm{L-Glutamine,} 100 \mathrm{U} / \mathrm{mL}$ penicillin and $100 \mu \mathrm{g} / \mathrm{mL}$ streptomycin at $37{ }^{\circ} \mathrm{C}$ and $5 \% \mathrm{CO}_{2}$. Prior to imaging the cells were briefly washed $3 \times$ with dye-free media. These cells were imaged using a Zeiss Elyra microscope equipped with $561 \mathrm{~nm}$ laser excitation and a $100 \times$ Plan-Apochromat 1.45 NA objective (Zeiss) and EMCCD (Andor). The cells were illuminated using oblique illumination and spt- $d$ STORM was performed as previously described. ${ }^{9}$ The emitted light was collected with a multi-bandpass filter (BP420-480 + BP570-640 + LP740). The single particles were imaged at $20 \mathrm{~Hz}$ and trajectories were mapped using TrackMate. The average trajectory length (i.e., duration time) per cell was quantified from all the trajectories with the maximum displacement $\mathrm{r}<400 \mathrm{~nm}$ within a single step. SPT intensity was quantified using Zeiss Zen Black for the average intensity of all single-particle emitters inside the cells. For SPT intensity, $n=7$ cells using $\mathbf{2 0}$ and $n=8$ cells using 21. For SPT duration time, $n=7$ cells using 20 and $n=16$ cells using 21. 
Single-particle tracking (SPT) experiments (Figure 4d,e, Figure 5d,e). SPT experiments were carried out as previously described. ${ }^{10}$ JM8.N4 mouse embryonic stem cells (ESCs) stably expressing histone H2B-HaloTag fusion proteins were generated by coexpressing the PiggyBac EF1a-H2B-HaloTag-IRES-Neo vector with the super piggyBac transposase as described previously, ${ }^{11}$ followed by G418 selection $(500 \mu \mathrm{g} / \mathrm{mL})$ for 2 weeks and verified by FACS sorting/confocal imaging staining with $\mathrm{JF}_{549}$-HaloTag ligand (20;100 nM, $\left.30 \mathrm{~min}\right)$. Cells were seeded on $25 \mathrm{~mm} \# 1.5$ coverglass pre-cleaned with $\mathrm{KOH}$ and ethanol and coated with Matrigel according to the manufacturer's instruction. All live cell imaging experiments were conducted using an ESC imaging medium composed of FluoroBrite DMEM (Thermo Fisher Scientific) plus 15\% v/v ESC-qualified FBS, 1× GlutaMax, 1× NEAA, 0.1 mM 2-mercaptoethanol, and LIF. To achieve sparse labeling, the histone H2B-HaloTag ESCs were stained with $2 \mathrm{nM}$ of HaloTag ligands 20 , 20D, 21D, 30, 30D, or 31 for $15 \mathrm{~min}$ at $37^{\circ} \mathrm{C}$ with $5 \% \mathrm{v} / \mathrm{v} \mathrm{CO}$ and then washed with dye-free media $3 \times$ for $30 \mathrm{~min}$ each wash. To quantify the brightness and photostability of the HaloTag ligands, labeled cells were mounted onto a high speed motorized Nikon Eclipse Ti-E inverted microscope equipped with the following: 100× Apo TIRF 1.49 NA objective with a correction collar; four excitation laser lines (405/488/561/642 nm) and matching TIRF quad cube (405/488/561/640 nm reflection bands); automatic TIRF illuminator with motorized X axis and manual $\mathrm{Y}$ axis for beam positioning and focus: perfect focus 3 system; Triple DU-897 iXon Ultra EMCCD cameras on a Cairn Tri-cam emission splitter with filters 525/50 (GFP) 600/50 (RFP) and 705/72 (Cy5); humidified incubation chamber maintained at $37^{\circ} \mathrm{C}$ with $5 \% \mathrm{v} / \mathrm{v} \mathrm{CO}_{2}$ (Tokai Hit). SPT was performed at $2 \mathrm{~Hz}$ for $\sim 500$ frames using ${ }^{~} 10 \%$ of maximal excitation power. The excitation laser was controlled by an acousto-optic tunable filter (AOTF) and reflected into the objective by a multi-band dichroic (405/488/561/633 BrightLine quad-band bandpass filter; Semrock). HaloTag ligands 20, 20D, and 21D were illuminated with $561 \mathrm{~nm}$ laser, whereas 30, 30D, and 31D were excited with a $641 \mathrm{~nm}$ laser. To minimize drift during imaging, the incubation chamber was fully thermally equilibrated and all imaging was conducted in an isolated ultra-clean room with minimal mechanical vibrations. The microscope, laser lines, and camera integration were controlled using the Nikon NIS-Elements software. We performed two independent biological replicates each with at least eight cells. Cells with obvious movement during acquisition were removed from further analysis. SMT analysis was based on multiple target tracing ${ }^{12}$ (MTT) with tracking parameters similar to previous work. ${ }^{10}$ For SPT brightness (kilocounts per second, kcps), intensity values from the first frame of the imaging experiment were analyzed: $n=3351$ single-molecule events using 20; $n=3838$ single-molecule events using 20 ; $n$ $=4724$ single-molecule events using 21 $;$ using 30D; $n=7125$ single-molecule events using 31 $\mathbf{D}$. For SPT duration (s), the single-molecule trajectories of each cell were combined and a survival curve was generated based on the 1-cumulative distribution function (1-CDF) histogram and mathematical fitting (MATLAB) as previously described. ${ }^{10}$ Histone H2B molecules were considered immobile under the imaging conditions and disappearance from the detection plane occurs primarily by photobleaching. Thus, the dissociation rate from this analysis equals the inverse of the fluorophore duration time, allowing comparison of photobleaching; $n=15$ cells using 20; $n=10$ cells using $\mathbf{2 0}$ D; $n=11$ cells using $\mathbf{2 1}$ D $; n=14$ cells using 30; $n=13$ cells using 30 $; \mathrm{n}=12$ cells using 31 $\mathbf{D}$. 
Fixed cell photobleaching experiments (Figure S5f,g, Figure S6c,d). U2OS cells expressing HaloTag-histone H2B fusion protein were co-fixed and labeled by incubation of 4\% w/v PFA and $200 \mathrm{nM}$ of ligands 20, 20D, 21, 21D, 30, 30D, 31, or 31D in $0.1 \mathrm{M}$ phosphate buffer. Cells were washed $3 \times$ in phosphate-buffered saline and then imaged using a Leica SP8 Falcon confocal microscope with an HC PL-APO 86×/1.20 water objective. To measure photobleaching, we used the tunable white light laser (WLL) excitation on the Leica SP8 Falcon confocal microscope to excite dyes at their $\lambda_{\text {abs }}$ in constant power mode; $n=3$.

Imaging in yeast (Figure 5f,g, Figure S6e,f). A Saccharomyces cerevisiae strain expressing the late Golgi marker Sec7-iGFP-HaloTag fusion from the native locus was grown shaking at room temperature to mid-log phase in NSD. Cells were incubated with HaloTag ligands 30, 30D, or 31 $\mathbf{D}$ (1 $\mu$ M diluted from a $1 \mathrm{mM}$ stock in DMSO; $30 \mathrm{~min})$, filter washed to remove excess dye, and adhered to a concanavalin A coated confocal dish. The cells were then imaged on a Leica SP8 laser scanning confocal microscope. GFP was excited at $488 \mathrm{~nm}$ and emission light was collected from 495-550 nm. Fluorophores 30, 30D, or 31 Dere excited at $633 \mathrm{~nm}$ and collected from 650-795 nm under identical conditions. Movie S1 is a combination of representative average projected z-stacks of cells labeled with the indicated dye. Deconvolution using Huygens Essential X11 software was performed to smooth the noisy confocal data. Average brightness per cell was calculated by measuring the total fluorescence intensity of the first frame of the movies and dividing by the total number of cells. The GFP fluorescence intensity was used as an internal control to correct for variation in expression between cells; $n=25$ for 30; $n=35$ for 30 $; n=37$ for 31. . Photobleaching curves were generated via the EMBL bleach correction plug-in on ImageJ. The graph consists of the average photobleaching traces of movies $(n=5)$ representing $25-40$ cells per condition.

Statistics and Reproducibility. For spectroscopy measurements, photobleaching experiments, or photo- and chromostability evaluations, reported $n$ values represent measurements of different samples prepared from the same dye DMSO stock solution or HaloTag conjugate stock solution. For cell loading experiments, $n$ represents the number of intensity values from individual nuclei extracted from three random fields of view at the indicated time points. For initial comparison of HaloTag ligands 20 and 21 in SPT, $n$ indicates separate cells. For the comparison of the SPT intensity of HaloTag ligands 20, 20, and 21 algorithm. For full comparison of the SPT duration time of HaloTag ligands 20, 20 $\mathbf{D}$, and 21 $\mathbf{D}$, or 30, 30 $\mathbf{D}$, and 31,$n$ indicates values extracted from separate cells. For cell-loading experiments, $n$ represents the number of intensity values from individual cells extracted from three fields of view at the indicated time points. For fluorescence microscopy experiments, all procedures were repeated at least once on a separate biological sample to ensure results were similar. Statistical analyses were performed using GraphPad Prism. P values were indicated as follows: 0.1234 (ns), $0.0332(*), 0.0021(* *), 0.0002(* *),<0.0001\left(^{* * * *)}\right.$. 


\section{GENERAL EXPERIMENTAL INFORMATION FOR SYNTHESIS}

Commercial reagents were obtained from reputable suppliers and used as received. All solvents were purchased in septum-sealed bottles stored under an inert atmosphere. Fluorescein ditriflate ${ }^{1}$ (15), Oregon Green ditriflate ${ }^{1}$ (S11), 6tert-butoxycarbonylfluorescein ditriflate ${ }^{3}$ (S1), carbofluorescein ditriflate ${ }^{2}$ (S13), Si-fluorescein ditriflate ${ }^{3}$ (S14), 4methylumbelliferone triflate $^{13}$ (S9), 10-acetyl-10H-phenoxazine-3,7-diyl bis(trifluoromethanesulfonate) ${ }^{3}$ (S10), and 6-tert-butoxycarbonyl-Si-fluorescein ditriflate $^{3}$ (S15) were synthesized as previously described. Azetidine$2,2,3,3,4,4-d_{6}$ was used as either the free base (Cambridge Isotopes) or the hydrochloride salt (synthesized as previously described $\left.{ }^{14}\right)$. All reactions were sealed with septa through which a nitrogen atmosphere was introduced unless otherwise noted. Reactions were conducted in round-bottomed flasks or septum-capped crimp-top vials containing Teflon-coated magnetic stir bars. Heating of reactions was accomplished with a silicon oil bath or an aluminum reaction block on top of a stirring hotplate equipped with an electronic contact thermometer to maintain the indicated temperatures.

Reactions were monitored by thin layer chromatography (TLC) on precoated TLC glass plates (silica gel $60 \mathrm{~F}_{254}, 250$ $\mu \mathrm{m}$ thickness) or by LC-MS (Phenomenex Kinetex $2.1 \mathrm{~mm} \times 30 \mathrm{~mm} 2.6 \mu \mathrm{m}$ C18 column; $5 \mu \mathrm{L}$ injection; 5-98\% $\mathrm{MeCN} / \mathrm{H}_{2} \mathrm{O}$, linear gradient, with constant $0.1 \% \mathrm{v} / \mathrm{v} \mathrm{HCO}_{2} \mathrm{H}$ additive; 6 min run; $0.5 \mathrm{~mL} / \mathrm{min}$ flow; ESI; positive ion mode). TLC chromatograms were visualized by UV illumination or developed with $p$-anisaldehyde, ceric ammonium molybdate, or $\mathrm{KMnO}_{4}$ stain. Reaction products were purified by flash chromatography on an automated purification system using pre-packed silica gel columns or by preparative HPLC (Phenomenex Gemini-NX $30 \times 150 \mathrm{~mm} 5 \mu \mathrm{m}$ C18 column). Analytical HPLC analysis was performed with an Agilent Eclipse XDB 4.6 $\times 150 \mathrm{~mm} 5 \mu \mathrm{m} \mathrm{C18} \mathrm{column}$ under the indicated conditions. High-resolution mass spectrometry was performed by the High Resolution Mass Spectrometry Facility at the University of Iowa.

NMR spectra were recorded on a $400 \mathrm{MHz}$ spectrometer. ${ }^{1} \mathrm{H}$ and ${ }^{13} \mathrm{C}$ chemical shifts were referenced to TMS or residual solvent peaks, and ${ }^{19} \mathrm{~F}$ chemical shifts were referenced to $\mathrm{CFCl}_{3}$. Data for ${ }^{1} \mathrm{H} \mathrm{NMR}$ spectra are reported as follows: chemical shift $(\delta \mathrm{ppm})$, multiplicity $(\mathrm{s}=$ singlet, $\mathrm{d}=$ doublet, $\mathrm{t}=$ triplet, $\mathrm{q}=$ quartet, $\mathrm{dd}=$ doublet of doublets, $\mathrm{m}=$ multiplet $)$, coupling constant $(\mathrm{Hz})$, integration. Data for ${ }^{13} \mathrm{C}$ NMR spectra are reported by chemical shift $(\delta \mathrm{ppm})$ with hydrogen multiplicity $\left(\mathrm{C}, \mathrm{CH}, \mathrm{CH}_{2}, \mathrm{CH}_{3}\right)$ information obtained from DEPT spectra. 


\section{EXPERIMENTALS}

\section{Pd-Catalyzed Cross-Coupling Reactions}

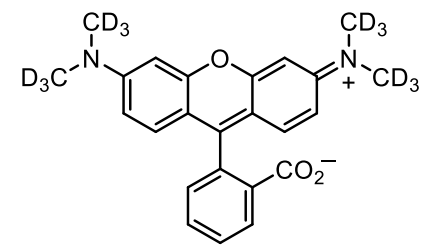

TMR- $\boldsymbol{d}_{\mathbf{1 2}}\left(\mathbf{1}_{\mathrm{D}}\right)$. A vial was charged with fluorescein ditriflate $(\mathbf{1 5}, 150 \mathrm{mg}, 0.251 \mathrm{mmol})$, dimethyl- $d_{6}$-amine hydrochloride (16, 52.9 mg, 0.604 mmol, 2.4 eq), $\mathrm{Pd}_{2} \mathrm{dba}_{3}(23.0 \mathrm{mg}, 25.1 \mu \mathrm{mol}, 0.1 \mathrm{eq})$, XPhos (36.0 mg, $75.4 \mu \mathrm{mol}$, $0.3 \mathrm{eq}$ ), and $\mathrm{Cs}_{2} \mathrm{CO}_{3}(393 \mathrm{mg}, 1.21 \mathrm{mmol}, 4.8 \mathrm{eq})$. The vial was sealed and evacuated/backfilled with nitrogen (3x). Dioxane $(1.5 \mathrm{~mL})$ was added, and the reaction was flushed again with nitrogen $(3 \times)$. The reaction was then stirred at $100{ }^{\circ} \mathrm{C}$ for $4 \mathrm{~h}$. It was subsequently cooled to room temperature, diluted with $\mathrm{MeOH}$, deposited onto Celite, and concentrated to dryness. Purification by silica gel chromatography (0-10\% $\mathrm{MeOH}\left(2 \mathrm{M} \mathrm{NH}_{3}\right) / \mathrm{CH}_{2} \mathrm{Cl}_{2}$, linear gradient; dry load on Celite) followed by reverse phase HPLC $\left(10-50 \% \mathrm{MeCN} / \mathrm{H}_{2} \mathrm{O}\right.$, linear gradient, with constant $0.1 \% \mathrm{v} / \mathrm{v}$ TFA additive) afforded $\mathbf{1}_{\mathbf{D}}$ (92 $\mathrm{mg}, 71 \%$, TFA salt) as a dark red solid. ${ }^{1} \mathrm{H}$ NMR $\left(\mathrm{CD}_{3} \mathrm{OD}, 400 \mathrm{MHz}\right) \delta 8.37-8.32$ (m, 1H), 7.86 (td, $J=7.5,1.5 \mathrm{~Hz}, 1 \mathrm{H}), 7.80$ (td, $J=7.6,1.5 \mathrm{~Hz}, 1 \mathrm{H}), 7.44-7.38(\mathrm{~m}, 1 \mathrm{H}), 7.15(\mathrm{~d}, J=9.5 \mathrm{~Hz}, 2 \mathrm{H})$, 7.05 (dd, $J=9.5,2.5 \mathrm{~Hz}, 2 \mathrm{H}), 6.97(\mathrm{~d}, J=2.5 \mathrm{~Hz}, 2 \mathrm{H}) ;{ }^{13} \mathrm{C}$ NMR $\left(\mathrm{CD}_{3} \mathrm{OD}, 101 \mathrm{MHz}\right) \delta 168.0(\mathrm{C}), 161.8(\mathrm{C}), 159.1$ (C), $159.0(\mathrm{C}), 135.3(\mathrm{C}), 133.8(\mathrm{CH}), 132.5(\mathrm{CH}), 132.3(\mathrm{C}), 132.1(\mathrm{CH}), 131.5(\mathrm{CH}), 131.4(\mathrm{CH}), 115.4(\mathrm{CH}), 115.0$ (C), $97.3(\mathrm{CH})$; Analytical HPLC: $\mathrm{t}_{\mathrm{R}}=10.5 \mathrm{~min},>99 \%$ purity $\left(10-95 \% \mathrm{MeCN} / \mathrm{H}_{2} \mathrm{O}\right.$, linear gradient, with constant $0.1 \% \mathrm{v} / \mathrm{v}$ TFA additive; $20 \mathrm{~min}$ run; $1 \mathrm{~mL} / \mathrm{min}$ flow; ESI; positive ion mode; detection at $550 \mathrm{~nm}$ ); HRMS (ESI) calcd for $\mathrm{C}_{24} \mathrm{H}_{11} \mathrm{D}_{12} \mathrm{~N}_{2} \mathrm{O}_{3}[\mathrm{M}+\mathrm{H}]^{+}$399.2456, found 399.2454.

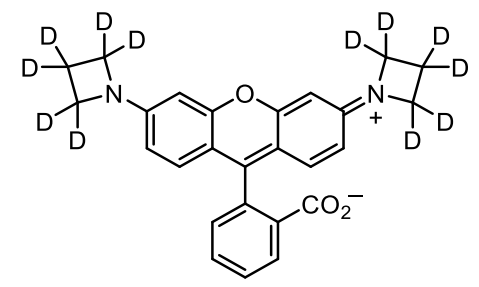

JFX $_{549}\left(\mathbf{6}_{\mathbf{D}}\right)$. A vial was charged with fluorescein ditriflate $(\mathbf{1 5}, 200 \mathrm{mg}, 0.335 \mathrm{mmol})$, azetidine-2,2,3,3,4,4- $d_{6}$

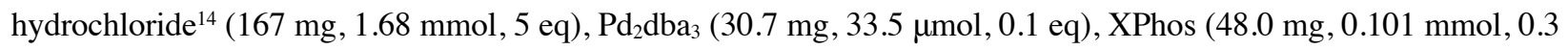
eq), and $\mathrm{Cs}_{2} \mathrm{CO}_{3}(874 \mathrm{mg}, 2.68 \mathrm{mmol}, 8 \mathrm{eq})$. The vial was sealed and evacuated/backfilled with nitrogen ( $3 \times$ ). Dioxane $(2 \mathrm{~mL})$ was added, and the reaction was flushed again with nitrogen $(3 \times)$. The reaction was then stirred at $100{ }^{\circ} \mathrm{C}$ for $2 \mathrm{~h}$. It was subsequently cooled to room temperature, diluted with $\mathrm{MeOH}$, deposited onto Celite, and concentrated to dryness. Purification by silica gel chromatography $\left(0-10 \% \mathrm{MeOH}\left(2 \mathrm{M} \mathrm{NH}_{3}\right) / \mathrm{CH}_{2} \mathrm{Cl}_{2}\right.$, linear gradient; dry load on Celite) afforded $\mathbf{6}_{\mathbf{D}}(115 \mathrm{mg}, 81 \%)$ as a dark red solid. ${ }^{1} \mathrm{H} \mathrm{NMR}\left(\mathrm{CDCl}_{3}, 400 \mathrm{MHz}\right) \delta 8.02-7.95(\mathrm{~m}, 1 \mathrm{H}), 7.63(\operatorname{td}, J$ $=7.4,1.3 \mathrm{~Hz}, 1 \mathrm{H}), 7.57(\mathrm{td}, J=7.4,1.1 \mathrm{~Hz}, 1 \mathrm{H}), 7.17(\mathrm{dt}, J=7.5,1.0 \mathrm{~Hz}, 1 \mathrm{H}), 6.56(\mathrm{~d}, J=8.6 \mathrm{~Hz}, 2 \mathrm{H}), 6.20(\mathrm{~d}, J=$ $2.3 \mathrm{~Hz}, 2 \mathrm{H}), 6.08$ (dd, $J=8.6,2.3 \mathrm{~Hz}, 2 \mathrm{H}) ;{ }^{13} \mathrm{C} \mathrm{NMR}\left(\mathrm{CDCl}_{3}, 101 \mathrm{MHz}\right) \delta 169.9(\mathrm{C}), 153.8(\mathrm{C}), 153.1$ (C), $153.0(\mathrm{C})$, 134.6 (CH), $129.4(\mathrm{CH}), 129.0(\mathrm{CH}), 127.8(\mathrm{C}), 125.0(\mathrm{CH}), 124.3(\mathrm{CH}), 107.9(\mathrm{C}), 107.8(\mathrm{CH}), 97.7(\mathrm{CH})$; Analytical 
HPLC: $t_{\mathrm{R}}=11.3 \mathrm{~min},>99 \%$ purity $\left(10-95 \% \mathrm{MeCN} / \mathrm{H}_{2} \mathrm{O}\right.$, linear gradient, with constant $0.1 \% \mathrm{v} / \mathrm{v}$ TFA additive; 20 min run; $1 \mathrm{~mL} / \mathrm{min}$ flow; ESI; positive ion mode; detection at $550 \mathrm{~nm}$ ); HRMS (ESI) calcd for $\mathrm{C}_{26} \mathrm{H}_{11} \mathrm{D}_{12} \mathrm{~N}_{2} \mathrm{O}_{3}[\mathrm{M}+\mathrm{H}]^{+}$ 423.2456, found 423.2454 .

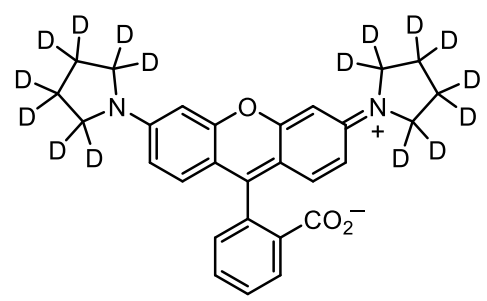

JFX $_{554}\left(\mathbf{5}_{\mathbf{D}}\right)$. A vial was charged with fluorescein ditriflate $(\mathbf{1 5}, 200 \mathrm{mg}, 0.335 \mathrm{mmol}), \mathrm{Pd}_{2} \mathrm{dba}_{3}(30.7 \mathrm{mg}, 33.5 \mu \mathrm{mol}$, $0.1 \mathrm{eq}$ ), XPhos (48.0 mg, $0.101 \mathrm{mmol}, 0.3 \mathrm{eq}$ ), and $\mathrm{Cs}_{2} \mathrm{CO}_{3}$ (306 mg, $0.939 \mathrm{mmol}, 2.8 \mathrm{eq}$ ). The vial was sealed and evacuated/backfilled with nitrogen $(3 \times)$. Dioxane $(2 \mathrm{~mL})$ was added, and the reaction was flushed again with nitrogen (3x). Following the addition of pyrrolidine-2,2,3,3,4,4,5,5- $d_{8}(64 \mathrm{mg}, 0.805 \mathrm{mmol}, 2.4 \mathrm{eq})$, the reaction was stirred at $100{ }^{\circ} \mathrm{C}$ for $3 \mathrm{~h}$. It was subsequently cooled to room temperature, diluted with $\mathrm{MeOH}$, deposited onto Celite, and concentrated to dryness. Purification by silica gel chromatography (0-10\% $\mathrm{MeOH}\left(2 \mathrm{M} \mathrm{NH}_{3}\right) / \mathrm{CH}_{2} \mathrm{Cl}_{2}$, linear gradient; dry load on Celite) afforded $\mathbf{5}_{\mathbf{D}}(116 \mathrm{mg}, 76 \%)$ as a dark red-purple solid. ${ }^{1} \mathrm{H} \mathrm{NMR}\left(\mathrm{CD}_{3} \mathrm{OD}, 400 \mathrm{MHz}\right) \delta 8.11-8.07$ (m, 1H), $7.65(\mathrm{td}, J=7.5,1.6 \mathrm{~Hz}, 1 \mathrm{H}), 7.60(\mathrm{td}, J=7.4,1.6 \mathrm{~Hz}, 1 \mathrm{H}), 7.26(\mathrm{~d}, J=9.3 \mathrm{~Hz}, 2 \mathrm{H}), 7.25-7.22(\mathrm{~m}, 1 \mathrm{H})$, $6.85(\mathrm{dd}, J=9.3,2.4 \mathrm{~Hz}, 2 \mathrm{H}), 6.75(\mathrm{~d}, J=2.3 \mathrm{~Hz}, 2 \mathrm{H}) ;{ }^{13} \mathrm{C} \mathrm{NMR}\left(\mathrm{CD}_{3} \mathrm{OD}, 101 \mathrm{MHz}\right) \delta 163.4(\mathrm{C}), 158.9(\mathrm{C}), 156.1$ (C), $141.8(\mathrm{C}), 133.9(\mathrm{C}), 132.9(\mathrm{CH}), 131.0(\mathrm{CH}), 130.7(\mathrm{CH}), 130.5(\mathrm{CH}), 130.4(\mathrm{CH}), 115.6(\mathrm{CH}), 115.1(\mathrm{C}), 97.5$ $(\mathrm{CH})$; Analytical HPLC: $\mathrm{t}_{\mathrm{R}}=12.4 \mathrm{~min},>99 \%$ purity $\left(10-95 \% \mathrm{MeCN} / \mathrm{H}_{2} \mathrm{O}\right.$, linear gradient, with constant $0.1 \% \mathrm{v} / \mathrm{v}$ TFA additive; $20 \mathrm{~min}$ run; $1 \mathrm{~mL} / \mathrm{min}$ flow; ESI; positive ion mode; detection at $550 \mathrm{~nm}$ ); HRMS (ESI) calcd for $\mathrm{C}_{28} \mathrm{H}_{11} \mathrm{D}_{16} \mathrm{~N}_{2} \mathrm{O}_{3}[\mathrm{M}+\mathrm{H}]^{+} 455.3020$, found 455.3018.

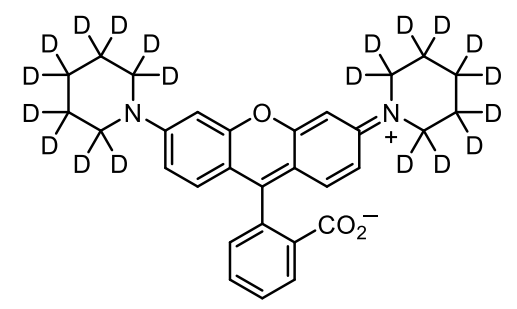

2-(3,6-Bis(piperidin-1-yl- $\left.\boldsymbol{d}_{\mathbf{1}}\right)$ xanthylium-9-yl)benzoate $\left(\mathbf{1 7}_{\mathbf{D}}\right)$. The title compound (96\%, dark red-purple solid) was prepared from fluorescein ditriflate (15) and piperidine-2,2,3,3,4,4,5,5,6,6- $d_{10}$ according to the procedure described for 5 5. $^{1} \mathrm{H}$ NMR $\left(\mathrm{CD}_{3} \mathrm{OD}, 400 \mathrm{MHz}\right) \delta 8.11-8.05(\mathrm{~m}, 1 \mathrm{H}), 7.66(\mathrm{td}, J=7.4,1.8 \mathrm{~Hz}, 1 \mathrm{H}), 7.62(\mathrm{td}, J=7.3,1.7 \mathrm{~Hz}$, $1 \mathrm{H}), 7.26-7.21(\mathrm{~m}, 1 \mathrm{H}), 7.17(\mathrm{~d}, J=9.4 \mathrm{~Hz}, 2 \mathrm{H}), 7.06(\mathrm{dd}, J=9.4,2.6 \mathrm{~Hz}, 2 \mathrm{H}), 7.01(\mathrm{~d}, J=2.5 \mathrm{~Hz}, 2 \mathrm{H}) ;{ }^{13} \mathrm{C} \mathrm{NMR}$ $\left(\mathrm{CD}_{3} \mathrm{OD}, 101 \mathrm{MHz}\right) \delta 158.9(\mathrm{C}), 157.6(\mathrm{C}), 152.1(\mathrm{C}), 139.7(\mathrm{C}), 136.7(\mathrm{C}), 132.6(\mathrm{CH}), 131.4(\mathrm{CH}), 130.8(\mathrm{CH})$, $130.3(\mathrm{CH}), 129.7(\mathrm{CH}), 115.1(\mathrm{CH}), 114.5(\mathrm{C}), 98.6(\mathrm{CH})$; Analytical HPLC: $\mathrm{t}_{\mathrm{R}}=12.7$ min, >99\% purity (10-95\% $\mathrm{MeCN} / \mathrm{H}_{2} \mathrm{O}$, linear gradient, with constant $0.1 \% \mathrm{v} / \mathrm{v}$ TFA additive; $20 \mathrm{~min}$ run; $1 \mathrm{~mL} / \mathrm{min}$ flow; ESI; positive ion mode; detection at $550 \mathrm{~nm}$ ); HRMS (ESI) calcd for $\mathrm{C}_{30} \mathrm{H}_{11} \mathrm{D}_{20} \mathrm{~N}_{2} \mathrm{O}_{3}[\mathrm{M}+\mathrm{H}]^{+} 487.3585$, found 487.3588. 


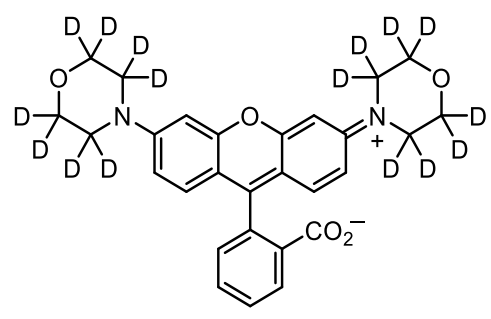

2-(3,6-Bis(morpholino- $\left.\boldsymbol{d}_{\mathbf{8}}\right)$ xanthylium-9-yl)benzoate $\left(\mathbf{1 8}_{\mathrm{D}}\right)$. The title compound (88\%, pink solid) was prepared from fluorescein ditriflate (15) and morpholine-2,2,3,3,5,5,6,6- $d_{8}$ according to the procedure described for $\mathbf{5}_{\mathbf{D}}$. ${ }^{1} \mathrm{H}$ $\operatorname{NMR}\left(\mathrm{CDCl}_{3}, 400 \mathrm{MHz}\right) \delta 8.04-7.98(\mathrm{~m}, 1 \mathrm{H}), 7.64(\mathrm{td}, J=7.4,1.4 \mathrm{~Hz}, 1 \mathrm{H}), 7.59(\mathrm{td}, J=7.4,1.2 \mathrm{~Hz}, 1 \mathrm{H}), 7.18-$ $7.13(\mathrm{~m}, 1 \mathrm{H}), 6.67(\mathrm{~d}, J=2.5 \mathrm{~Hz}, 2 \mathrm{H}), 6.65(\mathrm{~d}, J=8.8 \mathrm{~Hz}, 2 \mathrm{H}), 6.57(\mathrm{dd}, J=8.8,2.5 \mathrm{~Hz}, 2 \mathrm{H}) ;{ }^{13} \mathrm{C} \mathrm{NMR}\left(\mathrm{CDCl}_{3}, 101\right.$ MHz) $\delta 169.8(\mathrm{C}), 153.4(\mathrm{C}), 153.1(\mathrm{C}), 152.8(\mathrm{C}), 134.9(\mathrm{CH}), 129.6(\mathrm{CH}), 128.9(\mathrm{CH}), 127.2(\mathrm{C}), 125.0(\mathrm{CH}), 124.1$ $(\mathrm{CH}), 111.4(\mathrm{CH}), 109.9(\mathrm{C}), 101.8(\mathrm{CH}), 83.9(\mathrm{C})$; Analytical HPLC: $\mathrm{t}_{\mathrm{R}}=10.2 \mathrm{~min},>99 \%$ purity (10-95\% $\mathrm{MeCN} / \mathrm{H}_{2} \mathrm{O}$, linear gradient, with constant $0.1 \% \mathrm{v} / \mathrm{v}$ TFA additive; $20 \mathrm{~min}$ run; $1 \mathrm{~mL} / \mathrm{min}$ flow; ESI; positive ion mode; detection at $550 \mathrm{~nm}$ ); HRMS (ESI) calcd for $\mathrm{C}_{28} \mathrm{H}_{11} \mathrm{D}_{16} \mathrm{~N}_{2} \mathrm{O}_{5}[\mathrm{M}+\mathrm{H}]^{+} 487.2919$, found 487.2926.

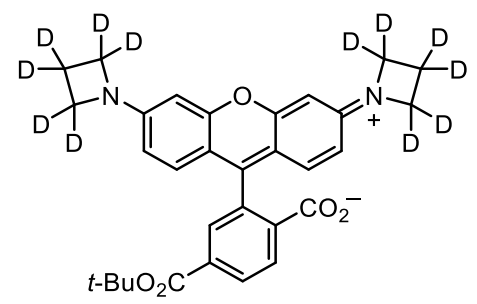

6-tert-Butoxycarbonyl-JFX ${ }_{549}$ (S2). The title compound (72\%, dark red-purple solid) was prepared from 6-tertbutoxycarbonylfluorescein ditriflate (S1) and azetidine-2,2,3,3,4,4- $d_{6}$ hydrochloride ${ }^{14}$ according to the procedure described for 6 $\mathbf{6}_{\mathbf{D}}{ }^{1} \mathrm{H} \mathrm{NMR}\left(\mathrm{CDCl}_{3}, 400 \mathrm{MHz}\right) \delta 8.19(\mathrm{dd}, J=8.0,1.3 \mathrm{~Hz}, 1 \mathrm{H}), 8.01(\mathrm{dd}, J=8.0,0.5 \mathrm{~Hz}, 1 \mathrm{H}), 7.77-$ $7.70(\mathrm{~m}, 1 \mathrm{H}), 6.53(\mathrm{~d}, J=8.6 \mathrm{~Hz}, 2 \mathrm{H}), 6.21(\mathrm{~d}, J=2.3 \mathrm{~Hz}, 2 \mathrm{H}), 6.09(\mathrm{dd}, J=8.6,2.3 \mathrm{~Hz}, 2 \mathrm{H}), 1.54(\mathrm{~s}, 9 \mathrm{H}) ;{ }^{13} \mathrm{C} \mathrm{NMR}$ $\left(\mathrm{CDCl}_{3}, 101 \mathrm{MHz}\right) \delta 169.1(\mathrm{C}), 164.5(\mathrm{C}), 153.8(\mathrm{C}), 153.3(\mathrm{C}), 152.9(\mathrm{C}), 138.1(\mathrm{C}), 130.8(\mathrm{C}), 130.6(\mathrm{CH}), 128.9$ $(\mathrm{CH}), 125.3(\mathrm{CH}), 124.8(\mathrm{CH}), 107.8(\mathrm{CH}), 107.2(\mathrm{C}), 97.7(\mathrm{CH}), 86.5(\mathrm{C}), 82.5(\mathrm{C}), 28.2\left(\mathrm{CH}_{3}\right)$; Analytical HPLC: $\mathrm{t}_{\mathrm{R}}=12.9 \mathrm{~min},>99 \%$ purity $\left(10-95 \% \mathrm{MeCN} / \mathrm{H}_{2} \mathrm{O}\right.$, linear gradient, with constant $0.1 \% \mathrm{v} / \mathrm{v}$ TFA additive; 20 min run; $1 \mathrm{~mL} / \mathrm{min}$ flow; ESI; positive ion mode; detection at $550 \mathrm{~nm}$ ); HRMS (ESI) calcd for $\mathrm{C}_{31} \mathrm{H}_{19} \mathrm{D}_{12} \mathrm{~N}_{2} \mathrm{O}_{5}[\mathrm{M}+\mathrm{H}]^{+}$ 523.2981, found 523.2982.

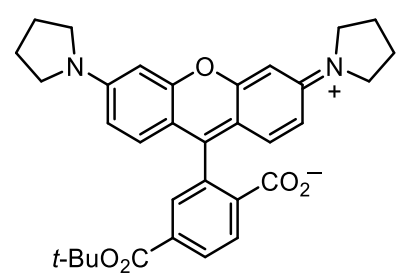

6-tert-Butoxycarbonyl-Rh $\mathbf{P}(\mathbf{S 3})$. The title compound (59\%, dark red-purple solid) was prepared from 6-tertbutoxycarbonylfluorescein ditriflate (S1) and pyrrolidine according to the procedure described for $\mathbf{5}_{\mathbf{D}}$. ${ }^{1} \mathrm{H}$ NMR $\left(\mathrm{CD}_{3} \mathrm{OD}, 400 \mathrm{MHz}\right) \delta 8.20(\mathrm{dd}, J=8.1,1.7 \mathrm{~Hz}, 1 \mathrm{H}), 8.11(\mathrm{~d}, J=8.1 \mathrm{~Hz}, 1 \mathrm{H}), 7.78(\mathrm{~d}, J=1.7 \mathrm{~Hz}, 1 \mathrm{H}), 7.22(\mathrm{~d}, J=$ 
$9.4 \mathrm{~Hz}, 2 \mathrm{H}), 6.87(\mathrm{dd}, J=9.4,2.3 \mathrm{~Hz}, 2 \mathrm{H}), 6.77(\mathrm{~d}, J=2.3 \mathrm{~Hz}, 2 \mathrm{H}), 3.65-3.52(\mathrm{~m}, 8 \mathrm{H}), 2.20-2.06(\mathrm{~m}, 8 \mathrm{H}), 1.59$ (s, 9H); ${ }^{13} \mathrm{C}$ NMR $\left(\mathrm{CD}_{3} \mathrm{OD}, 101 \mathrm{MHz}\right) \delta 172.4(\mathrm{C}), 166.1(\mathrm{C}), 162.2(\mathrm{C}), 158.9$ (C), $156.1(\mathrm{C}), 145.9(\mathrm{C}), 133.8$ (C), $133.6(\mathrm{C}), 132.7(\mathrm{CH}), 131.5(\mathrm{CH}), 131.2(\mathrm{CH}), 131.0(\mathrm{CH}), 115.9(\mathrm{CH}), 115.1(\mathrm{C}), 97.6(\mathrm{CH}), 83.1(\mathrm{C}), 49.9\left(\mathrm{CH}_{2}\right)$, $28.4\left(\mathrm{CH}_{3}\right), 26.2\left(\mathrm{CH}_{2}\right)$; Analytical HPLC: $\mathrm{t}_{\mathrm{R}}=13.7 \mathrm{~min},>99 \%$ purity $\left(10-95 \% \mathrm{MeCN} / \mathrm{H}_{2} \mathrm{O}\right.$, linear gradient, with constant $0.1 \% \mathrm{v} / \mathrm{v}$ TFA additive; $20 \mathrm{~min}$ run; $1 \mathrm{~mL} / \mathrm{min}$ flow; ESI; positive ion mode; detection at $550 \mathrm{~nm}$ ); HRMS (ESI) calcd for $\mathrm{C}_{33} \mathrm{H}_{35} \mathrm{~N}_{2} \mathrm{O}_{5}[\mathrm{M}+\mathrm{H}]^{+} 539.2540$, found 539.2544 .

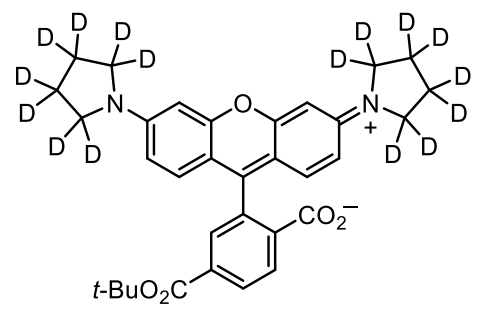

6-tert-Butoxycarbonyl-JFX $\mathbf{S 5 4}_{54}$ (S4). The title compound (58\%, dark red-purple solid) was prepared from 6-tertbutoxycarbonylfluorescein ditriflate (S1) and pyrrolidine-2,2,3,3,4,4,5,5- $d_{8}$ according to the procedure described for 5. ${ }^{1} \mathrm{H}$ NMR $\left(\mathrm{CD}_{3} \mathrm{OD}, 400 \mathrm{MHz}\right) \delta 8.20(\mathrm{dd}, J=8.2,1.7 \mathrm{~Hz}, 1 \mathrm{H}), 8.11(\mathrm{~d}, J=8.1 \mathrm{~Hz}, 1 \mathrm{H}), 7.78(\mathrm{~d}, J=1.6 \mathrm{~Hz}, 1 \mathrm{H})$, $7.21(\mathrm{~d}, J=9.3 \mathrm{~Hz}, 2 \mathrm{H}), 6.87(\mathrm{dd}, J=9.3,2.4 \mathrm{~Hz}, 2 \mathrm{H}), 6.76(\mathrm{~d}, J=2.3 \mathrm{~Hz}, 2 \mathrm{H}), 1.59$ (s, 9H); ${ }^{13} \mathrm{C} \mathrm{NMR}\left(\mathrm{CD}_{3} \mathrm{OD}, 101\right.$ MHz) $\delta 172.5$ (C), 166.1 (C), 162.2 (C), 158.9 (C), 156.2 (C), 145.9 (C), 133.7 (C), 133.6 (C), 132.7 (CH), 131.5 $(\mathrm{CH}), 131.2(\mathrm{CH}), 131.0(\mathrm{CH}), 115.9(\mathrm{CH}), 115.0(\mathrm{C}), 97.6(\mathrm{CH}), 83.1(\mathrm{C}), 28.4\left(\mathrm{CH}_{3}\right)$; Analytical HPLC: $\mathrm{t}_{\mathrm{R}}=13.6$ min, $>99 \%$ purity $\left(10-95 \% \mathrm{MeCN} / \mathrm{H}_{2} \mathrm{O}\right.$, linear gradient, with constant $0.1 \% \mathrm{v} / \mathrm{v}$ TFA additive; $20 \mathrm{~min} \mathrm{run} ; 1 \mathrm{~mL} / \mathrm{min}$ flow; ESI; positive ion mode; detection at $550 \mathrm{~nm}$ ); HRMS (ESI) calcd for $\mathrm{C}_{33} \mathrm{H}_{19} \mathrm{D}_{16} \mathrm{~N}_{2} \mathrm{O}_{5}[\mathrm{M}+\mathrm{H}]^{+} 555.3545$, found 555.3544.

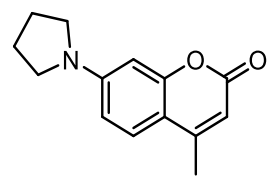

4-Methyl-7-(pyrrolidin-1-yl)-2H-chromen-2-one (22). A vial was charged with 4-methylumbelliferone triflate (S9;

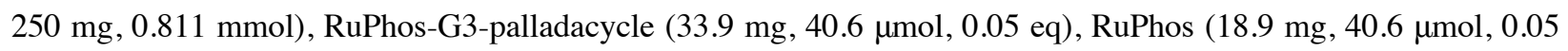
eq), and $\mathrm{K}_{2} \mathrm{CO}_{3}$ (157 mg, $1.14 \mathrm{mmol}, 1.4 \mathrm{eq}$ ). The vial was sealed and evacuated/backfilled with nitrogen $(3 \times)$. Dioxane $(4 \mathrm{~mL})$ was added, and the reaction was flushed again with nitrogen $(3 \times)$. Following the addition of pyrrolidine $(81.2 \mu \mathrm{L}, 0.973 \mathrm{mmol}, 1.2 \mathrm{eq})$, the reaction was stirred at $80{ }^{\circ} \mathrm{C}$ for $6 \mathrm{~h}$. It was subsequently cooled to room temperature, filtered through Celite with $\mathrm{CH}_{2} \mathrm{Cl}_{2}$, and concentrated in vacuo. Purification by silica gel chromatography (0-50\% EtOAc/hexanes, linear gradient) afforded $171 \mathrm{mg}(92 \%)$ of 22 as a yellow solid. ${ }^{1} \mathrm{H}$ NMR $\left(\mathrm{CDCl}_{3}, 400 \mathrm{MHz}\right) \delta 7.39(\mathrm{~d}, J=8.8 \mathrm{~Hz}, 1 \mathrm{H}), 6.48(\mathrm{dd}, J=8.8,2.4 \mathrm{~Hz}, 1 \mathrm{H}), 6.38(\mathrm{~d}, J=2.4 \mathrm{~Hz}, 1 \mathrm{H}), 5.94(\mathrm{q}, J=1.1$ $\mathrm{Hz}, 1 \mathrm{H}), 3.40-3.30(\mathrm{~m}, 4 \mathrm{H}), 2.34(\mathrm{~d}, J=1.1 \mathrm{~Hz}, 3 \mathrm{H}), 2.10-1.99(\mathrm{~m}, 4 \mathrm{H}) ;{ }^{13} \mathrm{C} \mathrm{NMR}\left(\mathrm{CDCl}_{3}, 101 \mathrm{MHz}\right) \delta 162.3$ (C), $155.9(\mathrm{C}), 153.2(\mathrm{C}), 150.5(\mathrm{C}), 125.5(\mathrm{CH}), 109.4(\mathrm{C}), 109.1(\mathrm{CH}), 108.8(\mathrm{CH}), 98.0(\mathrm{CH}), 47.8\left(\mathrm{CH}_{2}\right), 25.6$ $\left(\mathrm{CH}_{2}\right), 18.6\left(\mathrm{CH}_{3}\right)$; Analytical HPLC: $\mathrm{t}_{\mathrm{R}}=13.2 \mathrm{~min},>99 \%$ purity $\left(10-95 \% \mathrm{MeCN} / \mathrm{H}_{2} \mathrm{O}\right.$, linear gradient, with constant 
$0.1 \% \mathrm{v} / \mathrm{v}$ TFA additive; $20 \mathrm{~min}$ run; $1 \mathrm{~mL} / \mathrm{min}$ flow; ESI; positive ion mode; detection at $375 \mathrm{~nm}$ ); HRMS (ESI) calcd for $\mathrm{C}_{14} \mathrm{H}_{16} \mathrm{NO}_{2}[\mathrm{M}+\mathrm{H}]^{+} 230.1176$, found 230.1180.

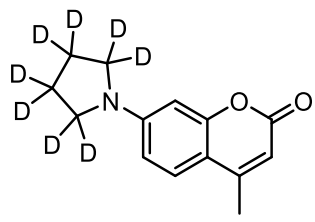

4-Methyl-7-(pyrrolidin-1-yl- $\left.\boldsymbol{d}_{\mathbf{8}}\right)$-2 $\boldsymbol{H}$-chromen-2-one $\left.\mathbf{( 2 2}_{\mathrm{D}}\right)$. The title compound (86\%, yellow solid) was prepared from 4-methylumbelliferone triflate (S9) and pyrrolidine-2,2,3,3,4,4,5,5- $d_{8}$ according to the procedure described for 22. ${ }^{1} \mathrm{H} \mathrm{NMR}\left(\mathrm{CDCl}_{3}, 400 \mathrm{MHz}\right) \delta 7.38(\mathrm{~d}, J=8.8 \mathrm{~Hz}, 1 \mathrm{H}), 6.48(\mathrm{dd}, J=8.8,2.4 \mathrm{~Hz}, 1 \mathrm{H}), 6.38(\mathrm{~d}, J=2.4 \mathrm{~Hz}, 1 \mathrm{H})$, $5.94(\mathrm{q}, J=1.1 \mathrm{~Hz}, 1 \mathrm{H}), 2.34(\mathrm{~d}, J=1.1 \mathrm{~Hz}, 3 \mathrm{H}) ;{ }^{13} \mathrm{C} \mathrm{NMR}\left(\mathrm{CDCl}_{3}, 101 \mathrm{MHz}\right) \delta 162.3(\mathrm{C}), 155.9(\mathrm{C}), 153.2(\mathrm{C})$, $150.6(\mathrm{C}), 125.5(\mathrm{CH}), 109.3(\mathrm{C}), 109.1(\mathrm{CH}), 108.7(\mathrm{CH}), 98.0(\mathrm{CH}), 18.6\left(\mathrm{CH}_{3}\right)$; Analytical HPLC: $t_{\mathrm{R}}=13.1 \mathrm{~min}$, $>99 \%$ purity $\left(10-95 \% \mathrm{MeCN} / \mathrm{H}_{2} \mathrm{O}\right.$, linear gradient, with constant $0.1 \% \mathrm{v} / \mathrm{v}$ TFA additive; $20 \mathrm{~min}$ run; $1 \mathrm{~mL} / \mathrm{min}$ flow; ESI; positive ion mode; detection at $375 \mathrm{~nm}$ ); HRMS (ESI) calcd for $\mathrm{C}_{14} \mathrm{H}_{8} \mathrm{D}_{8} \mathrm{NO}_{2}[\mathrm{M}+\mathrm{H}]^{+} 238.1678$, found 238.1682.

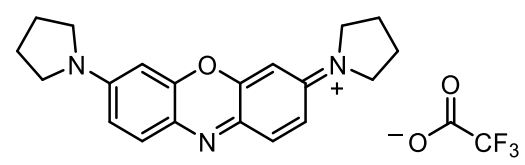

3,7-Di(pyrrolidin-1-yl)phenoxazin-5-ium trifluoroacetate (23). A vial was charged with 10-acetyl-10Hphenoxazine-3,7-diyl bis(trifluoromethanesulfonate) (S10; $250 \mathrm{mg}, 0.480 \mathrm{mmol}$ ), $\mathrm{Pd}_{2} \mathrm{dba}_{3}$ (43.9 mg, $48.0 \mu \mathrm{mol}, 0.1$ eq), XPhos (68.6 mg, $0.144 \mathrm{mmol}, 0.3 \mathrm{eq}$ ), and $\mathrm{Cs}_{2} \mathrm{CO}_{3}$ (437 mg, $1.34 \mathrm{mmol}, 2.8 \mathrm{eq}$ ). The vial was sealed and evacuated/backfilled with nitrogen $(3 \times)$. Dioxane $(2.5 \mathrm{~mL})$ was added, and the reaction was flushed again with nitrogen $(3 \times)$. Following the addition of pyrrolidine $(96.1 \mu \mathrm{L}, 1.15 \mathrm{mmol}, 2.4 \mathrm{eq})$, the reaction was stirred at $80{ }^{\circ} \mathrm{C}$ for $4 \mathrm{~h}$. It was then cooled to room temperature, filtered through Celite with $\mathrm{CH}_{2} \mathrm{Cl}_{2}$, and concentrated in vacuo. Purification by silica gel chromatography ( $0-40 \%$ EtOAc/toluene, linear gradient) afforded the $\mathrm{N}$-acetyl leuco-dye (112 $\mathrm{mg}, 64 \%)$ as an off-white solid.

The intermediate leuco-dye $(112 \mathrm{mg}, 0.308 \mathrm{mmol})$ was taken up in a mixture of $\mathrm{CH}_{2} \mathrm{Cl}_{2}(9 \mathrm{~mL})$ and water $(1 \mathrm{~mL})$. DDQ (105 mg, $0.462 \mathrm{mmol}, 1.5 \mathrm{eq}$ ) was added, and the reaction was stirred at room temperature for $2 \mathrm{~h}$. The crude reaction mixture was then deposited onto Celite and concentrated to dryness. Silica gel chromatography (0-20\% $\mathrm{MeOH} / \mathrm{CH}_{2} \mathrm{Cl}_{2}$, linear gradient, with constant $1 \% \mathrm{v} / \mathrm{v} \mathrm{AcOH}$ additive; dry load with Celite) followed by reverse phase HPLC (10-50\% MeCN/ $\mathrm{H}_{2} \mathrm{O}$, linear gradient, with constant $0.1 \% \mathrm{v} / \mathrm{v}$ TFA additive) afforded $127 \mathrm{mg}$ (95\%) of 23 as a dark blue solid. ${ }^{1} \mathrm{H}$ NMR $\left(\mathrm{CD}_{3} \mathrm{OD}, 400 \mathrm{MHz}\right) \delta 7.77(\mathrm{~d}, J=9.5 \mathrm{~Hz}, 2 \mathrm{H}), 7.25(\mathrm{dd}, J=9.4,2.5 \mathrm{~Hz}, 2 \mathrm{H}), 6.81(\mathrm{~d}, J=$ $2.5 \mathrm{~Hz}, 2 \mathrm{H}), 3.82-3.66(\mathrm{~m}, 8 \mathrm{H}), 2.22-2.13(\mathrm{~m}, 8 \mathrm{H}) ;{ }^{13} \mathrm{C} \mathrm{NMR}\left(\mathrm{CD}_{3} \mathrm{OD}, 101 \mathrm{MHz}\right) \delta 156.6(\mathrm{C}), 150.4(\mathrm{C}), 135.5$ (C), $135.3(\mathrm{CH}), 119.4(\mathrm{CH}), 98.0(\mathrm{CH}), 50.9\left(\mathrm{bs}, \mathrm{CH}_{2}\right), 26.2\left(\mathrm{bs}, \mathrm{CH}_{2}\right)$; Analytical HPLC: $\mathrm{t}_{\mathrm{R}}=10.4 \mathrm{~min},>99 \%$ purity (10-95\% MeCN/ $\mathrm{H}_{2} \mathrm{O}$, linear gradient, with constant $0.1 \%$ v/v TFA additive; $20 \mathrm{~min}$ run; $1 \mathrm{~mL} / \mathrm{min}$ flow; ESI; positive ion mode; detection at $650 \mathrm{~nm}$ ); HRMS (ESI) calcd for $\mathrm{C}_{20} \mathrm{H}_{22} \mathrm{~N}_{3} \mathrm{O}[\mathrm{M}]^{+} 320.1757$, found 320.1763 . 


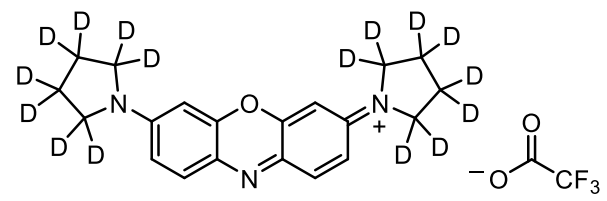

3,7-Bis(pyrrolidin-1-yl- $\boldsymbol{d}_{\mathbf{8}}$ )phenoxazin-5-ium trifluoroacetate $\left(\mathbf{2 3}_{\mathrm{D}}\right)$. The title compound (34\% 2-step yield, dark blue solid) was prepared from 10-acetyl-10H-phenoxazine-3,7-diyl bis(trifluoromethanesulfonate) (S10) and pyrrolidine-2,2,3,3,4,4,5,5- $d_{8}$ according to the procedure described for $23 .{ }^{1} \mathrm{H}$ NMR $\left(\mathrm{CD}_{3} \mathrm{OD}, 400 \mathrm{MHz}\right) \delta 7.77(\mathrm{~d}, J$ $=9.4 \mathrm{~Hz}, 2 \mathrm{H}), 7.25(\mathrm{dd}, J=9.5,2.5 \mathrm{~Hz}, 2 \mathrm{H}), 6.80(\mathrm{~d}, J=2.5 \mathrm{~Hz}, 2 \mathrm{H}) ;{ }^{13} \mathrm{C} \mathrm{NMR}\left(\mathrm{CD}_{3} \mathrm{OD}, 101 \mathrm{MHz}\right) \delta 156.7(\mathrm{C})$, $150.4(\mathrm{C}), 135.5(\mathrm{C}), 135.3(\mathrm{CH}), 119.4(\mathrm{CH}), 97.9(\mathrm{CH})$; Analytical HPLC: $\mathrm{t}_{\mathrm{R}}=10.3 \mathrm{~min},>99 \%$ purity $(10-95 \%$ $\mathrm{MeCN} / \mathrm{H}_{2} \mathrm{O}$, linear gradient, with constant $0.1 \% \mathrm{v} / \mathrm{v}$ TFA additive; $20 \mathrm{~min}$ run; $1 \mathrm{~mL} / \mathrm{min}$ flow; ESI; positive ion mode; detection at $650 \mathrm{~nm}$ ); HRMS (ESI) calcd for $\mathrm{C}_{20} \mathrm{H}_{6} \mathrm{D}_{16} \mathrm{~N}_{3} \mathrm{O}[\mathrm{M}]^{+} 336.2762$, found 336.2765.

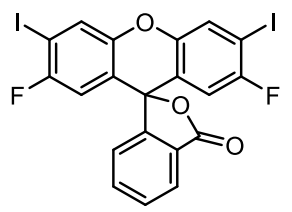

2',7'-Difluoro-3',6'-diiiodofluoran (S12). A vial was charged with Oregon Green ditriflate $\mathbf{S 1 1}$ (5.00 g, $7.91 \mathrm{mmol})$, tetrabutylammonium iodide (17.52 g, $47.44 \mathrm{mmol}, 6 \mathrm{eq})$, and [RuCp*(MeCN)$\left.)_{3}\right] \mathrm{OTf}(201 \mathrm{mg}, 0.395 \mathrm{mmol}, 0.05 \mathrm{eq})$. The vial was sealed and evacuated/backfilled with nitrogen $(3 \times)$. DMI $(40 \mathrm{~mL})$ was added, and the reaction was flushed again with nitrogen $(3 \times)$. The reaction was stirred at $100^{\circ} \mathrm{C}$ for $18 \mathrm{~h}$. It was then cooled to room temperature, diluted with water, and extracted with $3: 1 \mathrm{CHCl}_{3} /$ hexanes $(3 \times)$. The combined organics were washed with water and brine, dried over anhydrous $\mathrm{MgSO}_{4}$, filtered, and concentrated in vacuo. The crude material was purified twice by silica gel chromatography (0-10\% EtOAc/hexanes, linear gradient, with constant $40 \% \mathrm{v} / \mathrm{v} \mathrm{CH}_{2} \mathrm{Cl}_{2}$ additive; then, $100 \% \mathrm{CH}_{2} \mathrm{Cl}_{2}$ ) to yield $4.00 \mathrm{~g}$ of $\mathbf{S 1 2}(86 \%)$ as a white solid. ${ }^{1} \mathrm{H}$ NMR (DMSO- $\left.d_{6}, 400 \mathrm{MHz}\right) \delta 8.06-8.01(\mathrm{~m}, 1 \mathrm{H}$ ), $7.94\left(\mathrm{~d},{ }^{4} J_{\mathrm{HF}}=5.2 \mathrm{~Hz}, 2 \mathrm{H}\right), 7.80(\mathrm{td}, J=7.4,1.4 \mathrm{~Hz}, 1 \mathrm{H}), 7.75(\mathrm{td}, J=7.4,1.2 \mathrm{~Hz}, 1 \mathrm{H}), 7.38-7.33(\mathrm{~m}, 1 \mathrm{H}), 6.77(\mathrm{~d}$, $\left.{ }^{3} J_{\mathrm{HF}}=8.0 \mathrm{~Hz}, 2 \mathrm{H}\right) ;{ }^{19} \mathrm{~F}$ NMR (DMSO- $\left.d_{6}, 376 \mathrm{MHz}\right) \delta-100.10\left(\mathrm{dd}, J_{\mathrm{FH}}=8.0,5.2 \mathrm{~Hz}\right) ;{ }^{13} \mathrm{C}$ NMR (DMSO- $d 6,101$ MHz) $\delta 168.0(\mathrm{C}), 157.5$ (d, $\left.{ }^{1} J_{\mathrm{CF}}=239.5 \mathrm{~Hz}, \mathrm{CF}\right), 151.4(\mathrm{C}), 146.71\left(\mathrm{~d},{ }^{4} J_{\mathrm{CF}}=2.4 \mathrm{~Hz}, \mathrm{C}\right), 136.0(\mathrm{CH}), 130.7(\mathrm{CH})$, $127.4\left(\mathrm{~d},{ }^{3} J_{\mathrm{CF}}=2.4 \mathrm{~Hz}, \mathrm{CH}\right), 125.5(\mathrm{CH}), 125.0(\mathrm{C}), 123.7(\mathrm{CH}), 119.44\left(\mathrm{~d},{ }^{3} J_{\mathrm{CF}}=6.9 \mathrm{~Hz}, \mathrm{C}\right), 113.2\left(\mathrm{~d},{ }^{2} J_{\mathrm{CF}}=27.5\right.$ $\mathrm{Hz}, \mathrm{CH}), 86.0\left(\mathrm{~d},{ }^{2} J_{\mathrm{CF}}=29.0 \mathrm{~Hz}, \mathrm{C}\right), 80.2(\mathrm{C})$; HRMS (ESI) calcd for $\mathrm{C}_{20} \mathrm{H}_{9} \mathrm{~F}_{2} \mathrm{I}_{2} \mathrm{O}_{3}[\mathrm{M}+\mathrm{H}]^{+} 588.8604$, found 588.8613 .

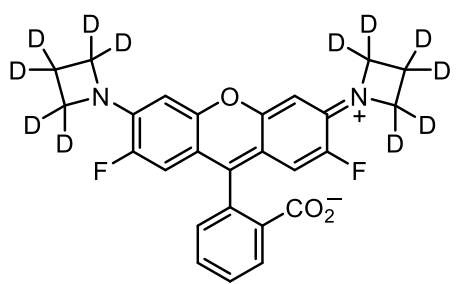

JFX $_{552}$ (24D). The title compound (66\%, dark red-purple solid, TFA salt) was prepared from 2',7'-difluoro-3',6'diiodofluoran (S12) and azetidine-2,2,3,3,4,4- $d_{6}$ according to the procedure described for $\mathbf{5}_{\mathbf{D}}{ }^{1}{ }^{1} \mathrm{H}$ NMR $\left(\mathrm{CD}_{3} \mathrm{OD}, 400\right.$ MHz) $\delta 8.37-8.33(\mathrm{~m}, 1 \mathrm{H}), 7.86(\mathrm{td}, J=7.5,1.6 \mathrm{~Hz}, 1 \mathrm{H}), 7.81(\mathrm{td}, J=7.6,1.6 \mathrm{~Hz}, 1 \mathrm{H}), 7.40-7.36(\mathrm{~m}, 1 \mathrm{H}), 6.73$ 
$\left(\mathrm{d},{ }^{3} J_{\mathrm{HF}}=12.7 \mathrm{~Hz}, 2 \mathrm{H}\right), 6.65\left(\mathrm{~d},{ }^{4} J_{\mathrm{HF}}=7.3 \mathrm{~Hz}, 2 \mathrm{H}\right) ;{ }^{19} \mathrm{~F}$ NMR $\left(\mathrm{CD}_{3} \mathrm{OD}, 376 \mathrm{MHz}\right) \delta-75.25(\mathrm{~s}, 3 \mathrm{~F}),-130.91\left(\mathrm{dd}, J_{\mathrm{FH}}\right.$ $=12.8,7.4 \mathrm{~Hz}, 2 \mathrm{~F}) ;{ }^{13} \mathrm{C} \mathrm{NMR}\left(\mathrm{CD}_{3} \mathrm{OD}, 101 \mathrm{MHz}\right) \delta 168.0(\mathrm{C}), 160.6(\mathrm{C}), 155.9(\mathrm{C}), 151.7\left(\mathrm{~d},{ }^{1} J_{\mathrm{CF}}=250.5 \mathrm{~Hz}, \mathrm{CF}\right)$, $148.9\left(\mathrm{~d},{ }^{2} J_{\mathrm{CF}}=15.2 \mathrm{~Hz}, \mathrm{C}\right), 134.9(\mathrm{C}), 134.2(\mathrm{CH}), 132.7(\mathrm{CH}), 131.93(\mathrm{C}), 131.86(\mathrm{CH}), 131.2(\mathrm{CH}), 114.59\left(\mathrm{~d},{ }^{3} J_{\mathrm{CF}}\right.$ $=8.5 \mathrm{~Hz}, \mathrm{C}), 113.7\left(\mathrm{~d},{ }^{2} J_{\mathrm{CF}}=21.6 \mathrm{~Hz}, \mathrm{CH}\right), 97.8\left(\mathrm{~d},{ }^{3} J_{\mathrm{CF}}=5.7 \mathrm{~Hz}, \mathrm{CH}\right)$; Analytical HPLC: $\mathrm{t}_{\mathrm{R}}=11.4 \mathrm{~min},>99 \%$ purity (10-95\% MeCN/ $\mathrm{H}_{2} \mathrm{O}$, linear gradient, with constant $0.1 \% \mathrm{v} / \mathrm{v}$ TFA additive; $20 \mathrm{~min}$ run; $1 \mathrm{~mL} / \mathrm{min}$ flow; ESI; positive ion mode; detection at $550 \mathrm{~nm}$ ); HRMS (ESI) calcd for $\mathrm{C}_{26} \mathrm{H}_{9} \mathrm{D}_{12} \mathrm{~F}_{2} \mathrm{~N}_{2} \mathrm{O}_{3}[\mathrm{M}+\mathrm{H}]^{+} 459.2268$, found 459.2272.

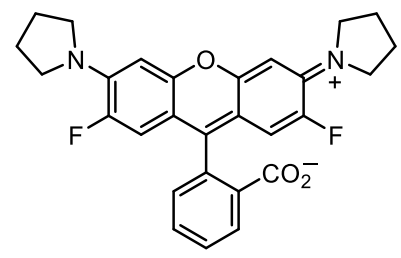

2',7'-Difluoro-RhP (25). The title compound (38\%, purple solid) was prepared from 2',7'-difluoro-3',6'-diiodofluoran (S12) and pyrrolidine according to the procedure described for 5D. $^{1} \mathrm{H}$ NMR $\left(\mathrm{CDCl}_{3}, 400 \mathrm{MHz}\right) \delta 8.01(\mathrm{dt}, J=7.4,1.1$ $\mathrm{Hz}, 1 \mathrm{H}), 7.67(\mathrm{td}, J=7.4,1.3 \mathrm{~Hz}, 1 \mathrm{H}), 7.61(\mathrm{td}, J=7.4,1.1 \mathrm{~Hz}, 1 \mathrm{H}), 7.17(\mathrm{dt}, J=7.5,1.1 \mathrm{~Hz}, 1 \mathrm{H}), 6.40\left(\mathrm{~d},{ }^{4} J_{\mathrm{HF}}=7.7\right.$ $\mathrm{Hz}, 2 \mathrm{H}), 6.28\left(\mathrm{~d},{ }^{3} J_{\mathrm{HF}}=14.0 \mathrm{~Hz}, 2 \mathrm{H}\right), 3.51-3.35(\mathrm{~m}, 8 \mathrm{H}), 2.01-1.88(\mathrm{~m}, 8 \mathrm{H}) ;{ }^{19} \mathrm{~F} \mathrm{NMR}\left(\mathrm{CDCl}_{3}, 376 \mathrm{MHz}\right) \delta-133.06$ - -133.17 (m); ${ }^{13} \mathrm{C}$ NMR $\left(\mathrm{CDCl}_{3}, 101 \mathrm{MHz}\right) \delta 169.5(\mathrm{C}), 152.7(\mathrm{C}), 148.8\left(\mathrm{~d},{ }^{4} J_{\mathrm{CF}}=1.0 \mathrm{~Hz}, \mathrm{C}\right), 148.2\left(\mathrm{~d},{ }^{1} J_{\mathrm{CF}}=238.5\right.$ $\mathrm{Hz}, \mathrm{CF}), 139.5\left(\mathrm{~d},{ }^{2} J_{\mathrm{CF}}=11.3 \mathrm{~Hz}, \mathrm{C}\right), 135.0(\mathrm{CH}), 129.8(\mathrm{CH}), 127.3(\mathrm{C}), 125.2(\mathrm{CH}), 124.1(\mathrm{CH}), 114.0\left(\mathrm{~d},{ }^{2} J_{\mathrm{CF}}=\right.$ $24.0 \mathrm{~Hz}, \mathrm{CH}), 105.2\left(\mathrm{~d},{ }^{3} J_{\mathrm{CF}}=6.7 \mathrm{~Hz}, \mathrm{C}\right), 101.6\left(\mathrm{~d},{ }^{3} J_{\mathrm{CF}}=5.5 \mathrm{~Hz}, \mathrm{CH}\right), 84.8(\mathrm{C}), 49.8\left(\mathrm{~d},{ }^{4} J_{\mathrm{CF}}=5.4 \mathrm{~Hz}, \mathrm{CH}_{2}\right), 25.5$ $\left(\mathrm{d},{ }^{5} J_{\mathrm{CF}}=2.1 \mathrm{~Hz}, \mathrm{CH}_{2}\right)$; Analytical HPLC: $\mathrm{t}_{\mathrm{R}}=12.3 \mathrm{~min},>99 \%$ purity $\left(10-95 \% \mathrm{MeCN} / \mathrm{H}_{2} \mathrm{O}\right.$, linear gradient, with constant $0.1 \% \mathrm{v} / \mathrm{v}$ TFA additive; $20 \mathrm{~min}$ run; $1 \mathrm{~mL} / \mathrm{min}$ flow; ESI; positive ion mode; detection at $550 \mathrm{~nm}$ ); HRMS (ESI) calcd for $\mathrm{C}_{28} \mathrm{H}_{25} \mathrm{~F}_{2} \mathrm{~N}_{2} \mathrm{O}_{3}[\mathrm{M}+\mathrm{H}]^{+} 475.1828$, found 475.1840 .

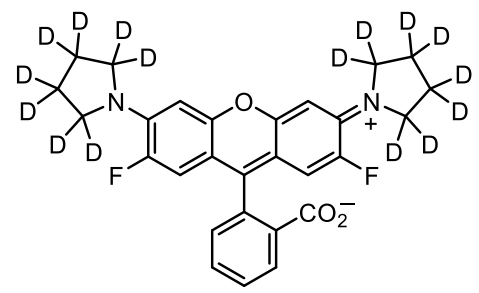

JFX $_{560}$ (25D). The title compound (62\%, purple solid) was prepared from 2',7'-difluoro-3',6'-diiodofluoran (S12) and pyrrolidine-2,2,3,3,4,4,5,5-d $d_{8}$ according to the procedure described for ${ }^{5} \mathbf{D} .{ }^{1} \mathrm{H} \mathrm{NMR}\left(\mathrm{CDCl}_{3}, 400 \mathrm{MHz}\right) \delta 8.04-7.97$ (m, 1H), 7.67 (td, $J=7.4,1.3 \mathrm{~Hz}, 1 \mathrm{H}), 7.61(\mathrm{td}, J=7.4,1.1 \mathrm{~Hz}, 1 \mathrm{H}), 7.17(\mathrm{dt}, J=7.5,0.9 \mathrm{~Hz}, 1 \mathrm{H}), 6.40\left(\mathrm{~d},{ }^{4} J_{\mathrm{HF}}=7.8\right.$ $\mathrm{Hz}, 2 \mathrm{H}), 6.28\left(\mathrm{~d},{ }^{3} J_{\mathrm{HF}}=14.0 \mathrm{~Hz}, 2 \mathrm{H}\right) ;{ }^{19} \mathrm{~F} \mathrm{NMR}\left(\mathrm{CDCl}_{3}, 376 \mathrm{MHz}\right) \delta-133.24\left(\mathrm{dd}, J_{\mathrm{FH}}=14.1,7.7 \mathrm{~Hz}\right) ;{ }^{13} \mathrm{C} \mathrm{NMR}$ $\left(\mathrm{CDCl}_{3}, 101 \mathrm{MHz}\right) \delta 169.5(\mathrm{C}), 152.6(\mathrm{C}), 148.9\left(\mathrm{~d},{ }^{4} J_{\mathrm{CF}}=0.8 \mathrm{~Hz}, \mathrm{C}\right), 148.2\left(\mathrm{~d},{ }^{1} J_{\mathrm{CF}}=238.5 \mathrm{~Hz}, \mathrm{CF}\right), 139.6\left(\mathrm{~d},{ }^{2} J_{\mathrm{CF}}\right.$ $=11.4 \mathrm{~Hz}, \mathrm{C}), 135.0(\mathrm{CH}), 129.8(\mathrm{CH}), 127.4(\mathrm{C}), 125.2(\mathrm{CH}), 124.1(\mathrm{CH}), 114.0\left(\mathrm{~d},{ }^{2} J_{\mathrm{CF}}=23.9 \mathrm{~Hz}, \mathrm{CH}\right), 105.1(\mathrm{~d}$, $\left.{ }^{3} J_{\mathrm{CF}}=6.7 \mathrm{~Hz}, \mathrm{C}\right), 101.5\left(\mathrm{~d},{ }^{3} J_{\mathrm{CF}}=5.4 \mathrm{~Hz}, \mathrm{CH}\right), 85.0(\mathrm{C})$; Analytical HPLC: $t_{\mathrm{R}}=12.3 \mathrm{~min},>99 \%$ purity $(10-95 \%$ $\mathrm{MeCN} / \mathrm{H}_{2} \mathrm{O}$, linear gradient, with constant $0.1 \% \mathrm{v} / \mathrm{v}$ TFA additive; $20 \mathrm{~min}$ run; $1 \mathrm{~mL} / \mathrm{min}$ flow; ESI; positive ion mode; detection at $550 \mathrm{~nm}$ ); HRMS (ESI) calcd for $\mathrm{C}_{28} \mathrm{H}_{9} \mathrm{D}_{16} \mathrm{~F}_{2} \mathrm{~N}_{2} \mathrm{O}_{3}[\mathrm{M}+\mathrm{H}]^{+} 491.2832$, found 491.2839. 


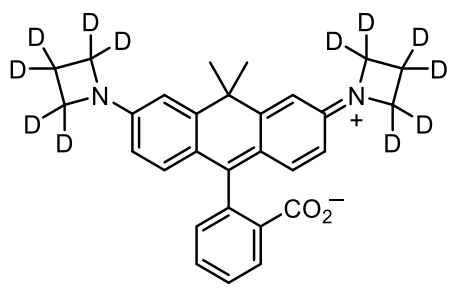

JFX $_{608}\left(\mathbf{2 6}_{\mathbf{D}}\right)$. The title compound (92\%, pale blue solid) was prepared from carbofluorescein ditriflate (S13) and azetidine-2,2,3,3,4,4- $d_{6}$ according to the procedure described for $\mathbf{5}_{\mathbf{D}} .{ }^{1} \mathrm{H}$ NMR $\left(\mathrm{CDCl}_{3}, 400 \mathrm{MHz}\right) \delta 8.00-7.95(\mathrm{~m}$, $1 \mathrm{H}), 7.58(\mathrm{td}, J=7.4,1.4 \mathrm{~Hz}, 1 \mathrm{H}), 7.53(\mathrm{td}, J=7.4,1.2 \mathrm{~Hz}, 1 \mathrm{H}), 7.08-7.04(\mathrm{~m}, 1 \mathrm{H}), 6.58(\mathrm{~d}, J=2.4 \mathrm{~Hz}, 2 \mathrm{H}), 6.55$ $(\mathrm{d}, J=8.6 \mathrm{~Hz}, 2 \mathrm{H}), 6.20(\mathrm{dd}, J=8.6,2.4 \mathrm{~Hz}, 2 \mathrm{H}), 1.82(\mathrm{~s}, 3 \mathrm{H}), 1.72(\mathrm{~s}, 3 \mathrm{H}) ;{ }^{13} \mathrm{C} \mathrm{NMR}\left(\mathrm{CDCl}_{3}, 101 \mathrm{MHz}\right) \delta 170.9$ (C), $155.5(\mathrm{C}), 152.5(\mathrm{C}), 146.9(\mathrm{C}), 134.5(\mathrm{CH}), 129.0(\mathrm{CH}), 128.9(\mathrm{CH}), 127.4(\mathrm{C}), 125.0(\mathrm{CH}), 124.1(\mathrm{CH}), 120.6$ (C), $110.4(\mathrm{CH}), 107.9(\mathrm{CH}), 88.5(\mathrm{C}), 38.5(\mathrm{C}), 35.7\left(\mathrm{CH}_{3}\right), 32.3\left(\mathrm{CH}_{3}\right)$; Analytical HPLC: $\mathrm{t}_{\mathrm{R}}=11.9 \mathrm{~min},>99 \%$ purity (10-95\% $\mathrm{MeCN} / \mathrm{H}_{2} \mathrm{O}$, linear gradient, with constant $0.1 \% \mathrm{v} / \mathrm{v}$ TFA additive; $20 \mathrm{~min}$ run; $1 \mathrm{~mL} / \mathrm{min}$ flow; ESI; positive ion mode; detection at $600 \mathrm{~nm}$ ); HRMS (ESI) calcd for $\mathrm{C}_{29} \mathrm{H}_{17} \mathrm{D}_{12} \mathrm{~N}_{2} \mathrm{O}_{2}[\mathrm{M}+\mathrm{H}]^{+} 449.2977$, found 449.2980 .

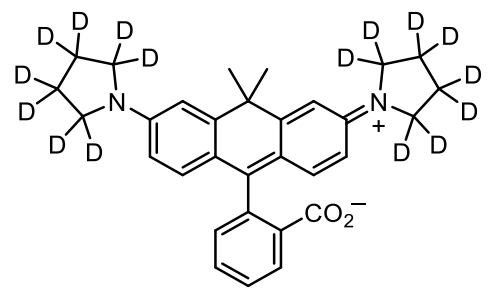

JFX $_{\mathbf{6 1 2}}\left(\mathbf{2 7}_{\mathbf{D}}\right)$. The title compound (91\%, pale blue solid) was prepared from carbofluorescein ditriflate (S13) and pyrrolidine-2,2,3,3,4,4,5,5- $d_{8}$ according to the procedure described for $\mathbf{5}_{\mathbf{D}}{ }^{1}{ }^{1} \mathrm{H} \mathrm{NMR}\left(\mathrm{CDCl}_{3}, 400 \mathrm{MHz}\right) \delta 8.00-7.96$ (m, 1H), 7.57 (td, $J=7.3,1.5 \mathrm{~Hz}, 1 \mathrm{H}), 7.52(\mathrm{td}, J=7.4,1.3 \mathrm{~Hz}, 1 \mathrm{H}), 7.08-7.04(\mathrm{~m}, 1 \mathrm{H}), 6.72(\mathrm{~d}, J=2.5 \mathrm{~Hz}, 2 \mathrm{H})$, $6.58(\mathrm{~d}, J=8.7 \mathrm{~Hz}, 2 \mathrm{H}), 6.35(\mathrm{dd}, J=8.7,2.5 \mathrm{~Hz}, 2 \mathrm{H}), 1.88(\mathrm{~s}, 3 \mathrm{H}), 1.77(\mathrm{~s}, 3 \mathrm{H}) ;{ }^{13} \mathrm{C} \mathrm{NMR}\left(\mathrm{CDCl}_{3}, 101 \mathrm{MHz}\right) \delta$ $171.1(\mathrm{C}), 155.7(\mathrm{C}), 148.3(\mathrm{C}), 147.2(\mathrm{C}), 134.4(\mathrm{CH}), 129.2(\mathrm{CH}), 128.7(\mathrm{CH}), 127.6(\mathrm{C}), 124.9(\mathrm{CH}), 124.1(\mathrm{CH})$, $118.8(\mathrm{C}), 111.0(\mathrm{CH}), 108.3(\mathrm{CH}), 38.6(\mathrm{C}), 36.0\left(\mathrm{CH}_{3}\right), 32.5\left(\mathrm{CH}_{3}\right)$; Analytical HPLC: $\mathrm{t}_{\mathrm{R}}=9.8 \mathrm{~min},>99 \%$ purity (30-95\% MeCN/ $\mathrm{H}_{2} \mathrm{O}$, linear gradient, with constant $0.1 \% \mathrm{v} / \mathrm{v}$ TFA additive; $20 \mathrm{~min}$ run; $1 \mathrm{~mL} / \mathrm{min}$ flow; ESI; positive ion mode; detection at $600 \mathrm{~nm}$ ); HRMS (ESI) calcd for $\mathrm{C}_{31} \mathrm{H}_{17} \mathrm{D}_{16} \mathrm{~N}_{2} \mathrm{O}_{2}[\mathrm{M}+\mathrm{H}]^{+} 481.3541$, found 481.3545 .

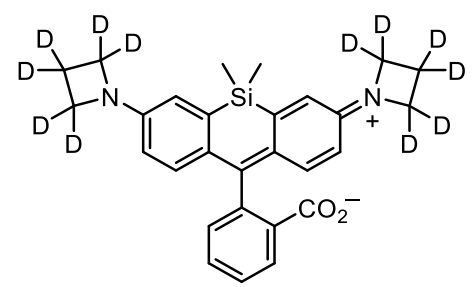

JFX $_{646}\left(\mathbf{2 8}_{\mathrm{D}}\right)$. The title compound (89\%, light blue solid) was prepared from Si-fluorescein ditriflate (S14) and azetidine-2,2,3,3,4,4- $d_{6}$ according to the procedure described for $\mathbf{5}_{\mathrm{D}}$. ${ }^{1} \mathrm{H} \mathrm{NMR}\left(\mathrm{CDCl}_{3}, 400 \mathrm{MHz}\right) \delta 7.96(\mathrm{dt}, J=7.6$, $1.0 \mathrm{~Hz}, 1 \mathrm{H}), 7.64(\mathrm{td}, J=7.5,1.1 \mathrm{~Hz}, 1 \mathrm{H}), 7.54(\mathrm{td}, J=7.5,0.8 \mathrm{~Hz}, 1 \mathrm{H}), 7.31(\mathrm{dt}, J=7.7,0.9 \mathrm{~Hz}, 1 \mathrm{H}), 6.75(\mathrm{~d}, J=$ $8.7 \mathrm{~Hz}, 2 \mathrm{H}), 6.67(\mathrm{~d}, J=2.6 \mathrm{~Hz}, 2 \mathrm{H}), 6.25(\mathrm{dd}, J=8.6,2.7 \mathrm{~Hz}, 2 \mathrm{H}), 0.61(\mathrm{~s}, 3 \mathrm{H}), 0.59(\mathrm{~s}, 3 \mathrm{H}) ;{ }^{13} \mathrm{C} \mathrm{NMR}\left(\mathrm{CDCl}_{3}, 101\right.$ 
MHz) $\delta 170.8(\mathrm{C}), 154.3(\mathrm{C}), 151.1(\mathrm{C}), 137.1(\mathrm{C}), 133.7(\mathrm{CH}), 132.9(\mathrm{C}), 128.8(\mathrm{CH}), 128.0(\mathrm{CH}), 127.2(\mathrm{C}), 125.8$ $(\mathrm{CH}), 124.8(\mathrm{CH}), 115.7(\mathrm{CH}), 112.3(\mathrm{CH}), 92.1(\mathrm{C}), 0.5\left(\mathrm{CH}_{3}\right),-1.5\left(\mathrm{CH}_{3}\right)$; Analytical HPLC: $\mathrm{t}_{\mathrm{R}}=12.5 \mathrm{~min},>99 \%$ purity $\left(10-95 \% \mathrm{MeCN} / \mathrm{H}_{2} \mathrm{O}\right.$, linear gradient, with constant $0.1 \% \mathrm{v} / \mathrm{v}$ TFA additive; $20 \mathrm{~min}$ run; $1 \mathrm{~mL} / \mathrm{min}$ flow; ESI; positive ion mode; detection at $650 \mathrm{~nm}$ ); HRMS (ESI) calcd for $\mathrm{C}_{28} \mathrm{H}_{17} \mathrm{D}_{12} \mathrm{~N}_{2} \mathrm{O}_{2} \mathrm{Si}[\mathrm{M}+\mathrm{H}]^{+} 465.2746$, found 465.2749.

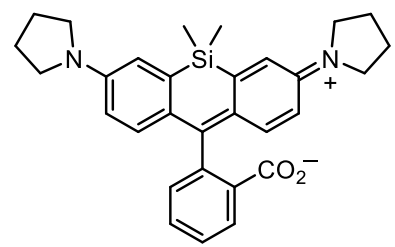

SiRh $_{\mathbf{P}}$ (29). The title compound (96\%, pale blue solid) was prepared from Si-fluorescein ditriflate (S14) and pyrrolidine according to the procedure described for $\mathbf{5}_{\mathbf{D}} .{ }^{1} \mathrm{H} \mathrm{NMR}\left(\mathrm{CDCl}_{3}, 400 \mathrm{MHz}\right) \delta 7.95(\mathrm{dt}, J=7.6,0.9 \mathrm{~Hz}, 1 \mathrm{H})$, $7.62(\mathrm{td}, J=7.5,1.1 \mathrm{~Hz}, 1 \mathrm{H}), 7.52(\mathrm{td}, J=7.5,0.9 \mathrm{~Hz}, 1 \mathrm{H}), 7.31-7.27(\mathrm{~m}, 1 \mathrm{H}), 6.79(\mathrm{~d}, J=2.7 \mathrm{~Hz}, 2 \mathrm{H}), 6.76(\mathrm{~d}, J$ $=8.8 \mathrm{~Hz}, 2 \mathrm{H}), 6.38(\mathrm{dd}, J=8.8,2.8 \mathrm{~Hz}, 2 \mathrm{H}), 3.34-3.24(\mathrm{~m}, 8 \mathrm{H}), 2.05-1.93(\mathrm{~m}, 8 \mathrm{H}), 0.63(\mathrm{~s}, 3 \mathrm{H}), 0.60(\mathrm{~s}, 3 \mathrm{H}) ;{ }^{13} \mathrm{C}$ NMR $\left(\mathrm{CDCl}_{3}, 101 \mathrm{MHz}\right) \delta 171.0(\mathrm{C}), 154.9$ (C), 146.8 (C), 137.2 (C), 133.7 (CH), 131.0 (C), $128.6(\mathrm{CH}), 128.4$ $(\mathrm{CH}), 127.2(\mathrm{C}), 125.6(\mathrm{CH}), 124.7(\mathrm{CH}), 115.9(\mathrm{CH}), 112.7(\mathrm{CH}), 92.5(\mathrm{C}), 47.6\left(\mathrm{CH}_{2}\right), 25.6\left(\mathrm{CH}_{2}\right), 0.6\left(\mathrm{CH}_{3}\right),-1.3$ $\left(\mathrm{CH}_{3}\right)$; Analytical HPLC: $\mathrm{t}_{\mathrm{R}}=13.0 \mathrm{~min},>99 \%$ purity $\left(10-95 \% \mathrm{MeCN} / \mathrm{H}_{2} \mathrm{O}\right.$, linear gradient, with constant $0.1 \% \mathrm{v} / \mathrm{v}$ TFA additive; $20 \mathrm{~min}$ run; $1 \mathrm{~mL} / \mathrm{min}$ flow; ESI; positive ion mode; detection at $650 \mathrm{~nm}$ ); HRMS (ESI) calcd for $\mathrm{C}_{30} \mathrm{H}_{33} \mathrm{~N}_{2} \mathrm{O}_{2} \mathrm{Si}[\mathrm{M}+\mathrm{H}]^{+} 481.2306$, found 481.2317.

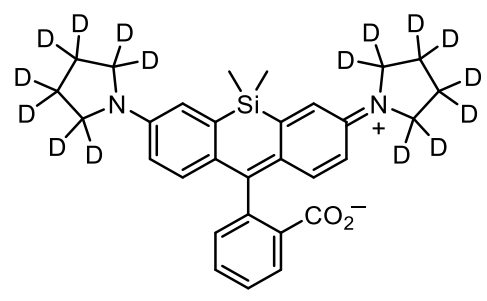

JFX $_{650}\left(\mathbf{2 9}_{\mathbf{D}}\right)$. The title compound (90\%, off-white solid) was prepared from Si-fluorescein ditriflate (S14) and pyrrolidine-2,2,3,3,4,4,5,5- $d_{8}$ according to the procedure described for $\mathbf{5}_{\mathbf{D}} \cdot{ }^{1} \mathrm{H}$ NMR $\left(\mathrm{CDCl}_{3}, 400 \mathrm{MHz}\right) \delta 7.95(\mathrm{dt}, J=$ 7.7, $1.0 \mathrm{~Hz}, 1 \mathrm{H}), 7.61(\mathrm{td}, J=7.5,1.1 \mathrm{~Hz}, 1 \mathrm{H}), 7.52(\mathrm{td}, J=7.5,0.9 \mathrm{~Hz}, 1 \mathrm{H}), 7.28(\mathrm{dt}, J=7.8,1.0 \mathrm{~Hz}, 1 \mathrm{H}), 6.79$ (d, $J$ $=2.8 \mathrm{~Hz}, 2 \mathrm{H}), 6.76(\mathrm{~d}, J=8.8 \mathrm{~Hz}, 2 \mathrm{H}), 6.38(\mathrm{dd}, J=8.8,2.8 \mathrm{~Hz}, 2 \mathrm{H}), 0.63(\mathrm{~s}, 3 \mathrm{H}), 0.59(\mathrm{~s}, 3 \mathrm{H}) ;{ }^{13} \mathrm{C} \mathrm{NMR}\left(\mathrm{CDCl}_{3}\right.$, $101 \mathrm{MHz}) \delta 171.0(\mathrm{C}), 154.9(\mathrm{C}), 147.0(\mathrm{C}), 137.2(\mathrm{C}), 133.7(\mathrm{CH}), 131.0(\mathrm{C}), 128.6(\mathrm{CH}), 128.5(\mathrm{CH}), 127.2(\mathrm{C})$, $125.7(\mathrm{CH}), 124.7(\mathrm{CH}), 115.9(\mathrm{CH}), 112.8(\mathrm{CH}), 92.5(\mathrm{C}), 0.6\left(\mathrm{CH}_{3}\right),-1.3\left(\mathrm{CH}_{3}\right)$; Analytical HPLC: $\mathrm{t}_{\mathrm{R}}=13.0 \mathrm{~min}$, $>99 \%$ purity $\left(10-95 \% \mathrm{MeCN} / \mathrm{H}_{2} \mathrm{O}\right.$, linear gradient, with constant $0.1 \% \mathrm{v} / \mathrm{v}$ TFA additive; $20 \mathrm{~min}$ run; $1 \mathrm{~mL} / \mathrm{min}$ flow; ESI; positive ion mode; detection at $650 \mathrm{~nm}$ ); HRMS (ESI) calcd for $\mathrm{C}_{30} \mathrm{H}_{17} \mathrm{D}_{16} \mathrm{~N}_{2} \mathrm{O}_{2} \mathrm{Si}[\mathrm{M}+\mathrm{H}]^{+}$497.3310, found 497.3312. 


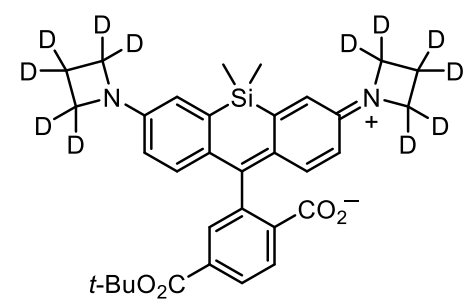

6-tert-Butoxycarbonyl-JFX ${ }_{646}$ (S16). The title compound (93\%, off-white solid) was prepared from 6-tertbutoxycarbonyl-Si-fluorescein ditriflate (S15) and azetidine-2,2,3,3,4,4- $d_{6}$ according to the procedure described for 5 ${ }^{1}{ }^{1} \mathrm{H}$ NMR $\left(\mathrm{CDCl}_{3}, 400 \mathrm{MHz}\right) \delta 8.11(\mathrm{dd}, J=8.0,1.3 \mathrm{~Hz}, 1 \mathrm{H}), 7.95(\mathrm{dd}, J=8.0,0.6 \mathrm{~Hz}, 1 \mathrm{H}), 7.83-7.80(\mathrm{~m}, 1 \mathrm{H})$, $6.82(\mathrm{~d}, J=8.7 \mathrm{~Hz}, 2 \mathrm{H}), 6.66(\mathrm{~d}, J=2.7 \mathrm{~Hz}, 2 \mathrm{H}), 6.29$ (dd, $J=8.7,2.7 \mathrm{~Hz}, 2 \mathrm{H}), 1.54$ (s, 9H), $0.64(\mathrm{~s}, 3 \mathrm{H}), 0.58$ (s, 3H); ${ }^{13} \mathrm{C} \mathrm{NMR}\left(\mathrm{CDCl}_{3}, 101 \mathrm{MHz}\right) \delta 170.3(\mathrm{C}), 164.5$ (C), 155.4 (C), 151.1 (C), 137.2 (C), 136.2 (C), $132.4(\mathrm{C}), 129.9$ $(\mathrm{CH}), 129.2(\mathrm{C}), 127.7(\mathrm{CH}), 125.6(\mathrm{CH}), 125.2(\mathrm{CH}), 115.7(\mathrm{CH}), 112.6(\mathrm{CH}), 91.9(\mathrm{C}), 82.3(\mathrm{C}), 28.2\left(\mathrm{CH}_{3}\right), 0.2$ $\left(\mathrm{CH}_{3}\right),-0.7\left(\mathrm{CH}_{3}\right)$; Analytical HPLC: $\mathrm{t}_{\mathrm{R}}=14.1 \mathrm{~min}, 98.8 \%$ purity $\left(10-95 \% \mathrm{MeCN} / \mathrm{H}_{2} \mathrm{O}\right.$, linear gradient, with constant $0.1 \% \mathrm{v} / \mathrm{v}$ TFA additive; $20 \mathrm{~min}$ run; $1 \mathrm{~mL} / \mathrm{min}$ flow; ESI; positive ion mode; detection at $650 \mathrm{~nm}$ ); HRMS (ESI) calcd for $\mathrm{C}_{33} \mathrm{H}_{25} \mathrm{D}_{12} \mathrm{~N}_{2} \mathrm{O}_{4} \mathrm{Si}[\mathrm{M}+\mathrm{H}]^{+}$565.3270, found 565.3277.

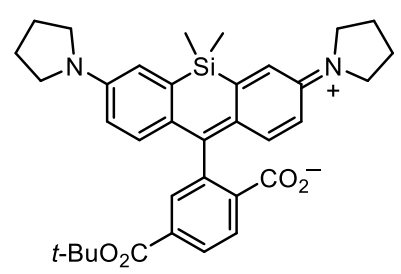

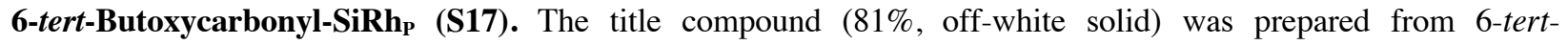
butoxycarbonyl-Si-fluorescein ditriflate (S15) and pyrrolidine according to the procedure described for $\mathbf{5}_{\mathbf{D}}$. ${ }^{1} \mathrm{H}$ NMR $\left(\mathrm{CDCl}_{3}, 400 \mathrm{MHz}\right) \delta 8.10(\mathrm{dd}, J=8.0,1.3 \mathrm{~Hz}, 1 \mathrm{H}), 7.96(\mathrm{dd}, J=8.0,0.5 \mathrm{~Hz}, 1 \mathrm{H}), 7.83-7.79(\mathrm{~m}, 1 \mathrm{H}), 6.84(\mathrm{~d}, J=$ $8.8 \mathrm{~Hz}, 2 \mathrm{H}), 6.79(\mathrm{~d}, J=2.7 \mathrm{~Hz}, 2 \mathrm{H}), 6.44(\mathrm{dd}, J=8.8,2.8 \mathrm{~Hz}, 2 \mathrm{H}), 3.35-3.25(\mathrm{~m}, 8 \mathrm{H}), 2.04-1.95(\mathrm{~m}, 8 \mathrm{H}), 1.53$ (s, 9H), 0.67 (s, 3H), 0.60 (s, 3H); ${ }^{13} \mathrm{C}$ NMR $\left(\mathrm{CDCl}_{3}, 101 \mathrm{MHz}\right) \delta 170.6$ (C), 164.5 (C), 155.9 (C), 146.8 (C), 137.2 $(\mathrm{C}), 136.3(\mathrm{C}), 130.5(\mathrm{C}), 129.8(\mathrm{CH}), 129.3(\mathrm{C}), 128.1(\mathrm{CH}), 125.5(\mathrm{CH}), 125.1(\mathrm{CH}), 115.9(\mathrm{CH}), 113.2(\mathrm{CH}), 92.2$ (C), 82.2 (C), $47.6\left(\mathrm{CH}_{2}\right), 28.2\left(\mathrm{CH}_{3}\right), 25.6\left(\mathrm{CH}_{2}\right), 0.2\left(\mathrm{CH}_{3}\right),-0.5\left(\mathrm{CH}_{3}\right)$; Analytical HPLC: $\mathrm{t}_{\mathrm{R}}=14.5 \mathrm{~min},>99 \%$ purity (10-95\% MeCN/ $\mathrm{H}_{2} \mathrm{O}$, linear gradient, with constant $0.1 \% \mathrm{v} / \mathrm{v}$ TFA additive; $20 \mathrm{~min}$ run; $1 \mathrm{~mL} / \mathrm{min}$ flow; ESI; positive ion mode; detection at $650 \mathrm{~nm}$ ); HRMS (ESI) calcd for $\mathrm{C}_{35} \mathrm{H}_{41} \mathrm{~N}_{2} \mathrm{O}_{4} \mathrm{Si}[\mathrm{M}+\mathrm{H}]^{+} 581.2830$, found 581.2839.

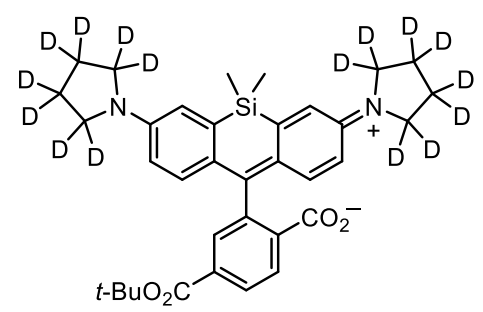

6-tert-Butoxycarbonyl-JFX $\mathbf{6 5 0}_{50}$ (S18). The title compound (87\%, off-white solid) was prepared from 6-tertbutoxycarbonyl-Si-fluorescein ditriflate (S15) and pyrrolidine-2,2,3,3,4,4,5,5- $d_{8}$ according to the procedure described 
for 5 5. $^{1} \mathrm{H} \mathrm{NMR}\left(\mathrm{CDCl}_{3}, 400 \mathrm{MHz}\right) \delta 8.10(\mathrm{dd}, J=8.0,1.3 \mathrm{~Hz}, 1 \mathrm{H}), 7.95(\mathrm{dd}, J=8.0,0.6 \mathrm{~Hz}, 1 \mathrm{H}), 7.83-7.78(\mathrm{~m}$, $1 \mathrm{H}), 6.84(\mathrm{~d}, J=8.8 \mathrm{~Hz}, 2 \mathrm{H}), 6.79(\mathrm{~d}, J=2.7 \mathrm{~Hz}, 2 \mathrm{H}), 6.43(\mathrm{dd}, J=8.8,2.8 \mathrm{~Hz}, 2 \mathrm{H}), 1.53(\mathrm{~s}, 9 \mathrm{H}), 0.67$ (s, 3H), 0.59 (s, 3H); ${ }^{13} \mathrm{C} \mathrm{NMR}\left(\mathrm{CDCl}_{3}, 101 \mathrm{MHz}\right) \delta 170.6$ (C), 164.5 (C), 155.9 (C), 146.9 (C), 137.2 (C), 136.3 (C), 130.5 (C), 129.8 (CH), $129.3(\mathrm{C}), 128.1(\mathrm{CH}), 125.5(\mathrm{CH}), 125.1(\mathrm{CH}), 115.9(\mathrm{CH}), 113.1(\mathrm{CH}), 92.3(\mathrm{C}), 82.2(\mathrm{C}), 28.2\left(\mathrm{CH}_{3}\right)$, $0.2\left(\mathrm{CH}_{3}\right),-0.5\left(\mathrm{CH}_{3}\right)$; Analytical HPLC: $\mathrm{t}_{\mathrm{R}}=14.4 \mathrm{~min},>99 \%$ purity $\left(10-95 \% \mathrm{MeCN} / \mathrm{H}_{2} \mathrm{O}\right.$, linear gradient, with constant $0.1 \% \mathrm{v} / \mathrm{v}$ TFA additive; $20 \mathrm{~min}$ run; $1 \mathrm{~mL} / \mathrm{min}$ flow; ESI; positive ion mode; detection at $650 \mathrm{~nm}$ ); HRMS (ESI) calcd for $\mathrm{C}_{35} \mathrm{H}_{25} \mathrm{D}_{16} \mathrm{~N}_{2} \mathrm{O}_{4} \mathrm{Si}[\mathrm{M}+\mathrm{H}]^{+}$597.3834, found 597.3835. 


\section{$t$-Butyl Ester Deprotection}

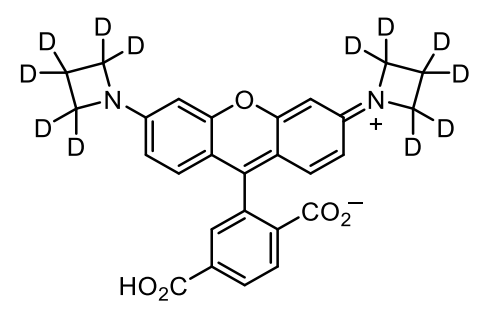

6-Carboxy-JFX ${ }_{549}$ (S5). 6-tert-Butoxycarbonyl-JFX ${ }_{549}(\mathbf{S 2} ; 102 \mathrm{mg}, 0.195 \mathrm{mmol})$ was taken up in $\mathrm{CH}_{2} \mathrm{Cl}_{2}(2.5 \mathrm{~mL})$, and trifluoroacetic acid $(0.5 \mathrm{~mL})$ was added. The reaction was stirred at room temperature for $6 \mathrm{~h}$. Toluene $(3 \mathrm{~mL})$ was added; the reaction mixture was concentrated to dryness and then azeotroped with $\mathrm{MeOH}$ three times to provide S5 as a red-brown solid (109 mg, 96\%, TFA salt). Analytical HPLC and NMR indicated that the material was $>95 \%$ pure and did not require further purification prior to amide coupling. ${ }^{1} \mathrm{H} N M R\left(\mathrm{CD}_{3} \mathrm{OD}, 400 \mathrm{MHz}\right) \delta 8.40(\mathrm{~d}, J=8.1$ $\mathrm{Hz}, 1 \mathrm{H}), 8.37$ (dd, $J=8.2,1.5 \mathrm{~Hz}, 1 \mathrm{H}), 7.95-7.93(\mathrm{~m}, 1 \mathrm{H}), 7.06(\mathrm{~d}, J=9.2 \mathrm{~Hz}, 2 \mathrm{H}), 6.60(\mathrm{dd}, J=9.2,2.2 \mathrm{~Hz}, 2 \mathrm{H})$, $6.54(\mathrm{~d}, J=2.2 \mathrm{~Hz}, 2 \mathrm{H}) ;{ }^{13} \mathrm{C} \mathrm{NMR}\left(\mathrm{CD}_{3} \mathrm{OD}, 101 \mathrm{MHz}\right) \delta 167.7$ (C), $167.3(\mathrm{C}), 160.0(\mathrm{C}), 158.8(\mathrm{C}), 158.2(\mathrm{C}), 136.0$ (C), $135.9(\mathrm{C}), 135.5(\mathrm{C}), 132.8(\mathrm{CH}), 132.3(\mathrm{CH}), 132.2(\mathrm{CH}), 114.8(\mathrm{C}), 113.6(\mathrm{CH}), 95.2(\mathrm{CH})$; Analytical HPLC: $\mathrm{t}_{\mathrm{R}}=9.9 \mathrm{~min},>99 \%$ purity $\left(10-95 \% \mathrm{MeCN} / \mathrm{H}_{2} \mathrm{O}\right.$, linear gradient, with constant $0.1 \% \mathrm{v} / \mathrm{v}$ TFA additive; $20 \mathrm{~min}$ run; 1 $\mathrm{mL} / \mathrm{min}$ flow; ESI; positive ion mode; detection at $550 \mathrm{~nm}$ ); HRMS (ESI) calcd for $\mathrm{C}_{27} \mathrm{H}_{11} \mathrm{D}_{12} \mathrm{~N}_{2} \mathrm{O}_{5}[\mathrm{M}+\mathrm{H}]^{+} 467.2355$, found 467.2354 .

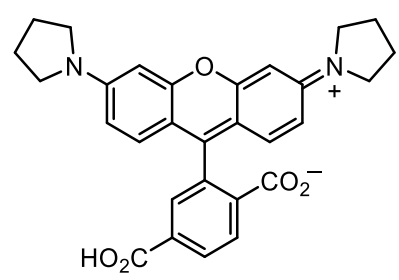

6-Carboxy-Rh $\mathbf{P}$ (S6). The title compound (99\%, dark red-purple solid, TFA salt) was prepared from 6-tertbutoxycarbonyl- $\mathrm{Rh}_{\mathrm{P}}(\mathbf{S 3})$ according to the procedure described for $\mathbf{S 5} .{ }^{1} \mathrm{H}$ NMR $\left(\mathrm{CD}_{3} \mathrm{OD}, 400 \mathrm{MHz}\right) \delta 8.42(\mathrm{~d}, J=$ $8.2 \mathrm{~Hz}, 1 \mathrm{H}), 8.39$ (dd, $J=8.2,1.5 \mathrm{~Hz}, 1 \mathrm{H}), 7.99-7.96(\mathrm{~m}, 1 \mathrm{H}), 7.11(\mathrm{~d}, J=9.4 \mathrm{~Hz}, 2 \mathrm{H}), 6.92(\mathrm{dd}, J=9.4,2.3 \mathrm{~Hz}$, $2 \mathrm{H}), 6.84(\mathrm{~d}, J=2.3 \mathrm{~Hz}, 2 \mathrm{H}), 3.68-3.56(\mathrm{~m}, 8 \mathrm{H}), 2.21-2.07(\mathrm{~m}, 8 \mathrm{H}) ;{ }^{13} \mathrm{C} \mathrm{NMR}\left(\mathrm{CD}_{3} \mathrm{OD}, 101 \mathrm{MHz}\right) \delta 167.7(\mathrm{C})$, $167.4(\mathrm{C}), 160.0(\mathrm{C}), 158.9(\mathrm{C}), 156.2(\mathrm{C}), 136.1(\mathrm{C}), 135.9(\mathrm{C}), 135.5(\mathrm{C}), 132.8(\mathrm{CH}), 132.31(\mathrm{CH}), 132.28(\mathrm{CH})$, $132.0(\mathrm{CH}), 116.4(\mathrm{CH}), 114.9(\mathrm{C}), 97.8(\mathrm{CH}), 50.0\left(\mathrm{CH}_{2}\right), 26.2\left(\mathrm{CH}_{2}\right)$; Analytical HPLC: $\mathrm{t}_{\mathrm{R}}=10.8 \mathrm{~min},>99 \%$ purity (5 $\mu \mathrm{L}$ injection; $10-95 \% \mathrm{MeCN} / \mathrm{H}_{2} \mathrm{O}$, linear gradient, with constant $0.1 \% \mathrm{v} / \mathrm{v}$ TFA additive; $20 \mathrm{~min} \mathrm{run} ; 1 \mathrm{~mL} / \mathrm{min}$ flow; ESI; positive ion mode; detection at $550 \mathrm{~nm}$ ); HRMS (ESI) calcd for $\mathrm{C}_{29} \mathrm{H}_{27} \mathrm{~N}_{2} \mathrm{O}_{5}[\mathrm{M}+\mathrm{H}]^{+} 483.1914$, found 483.1919 . 


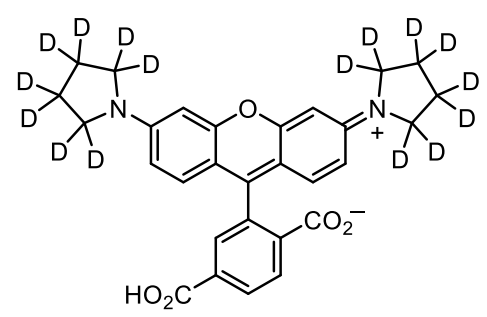

6-Carboxy-JFX ${ }_{554}$ (S7). The title compound (97\%, dark red-purple solid, TFA salt) was prepared from 6-tertbutoxycarbonyl-JFX 554 (S4) according to the procedure described for $\mathbf{S 5} .{ }^{1} \mathrm{H}$ NMR $\left(\mathrm{CD}_{3} \mathrm{OD}, 400 \mathrm{MHz}\right) \delta 8.41(\mathrm{~d}, J=$ $8.2 \mathrm{~Hz}, 1 \mathrm{H}), 8.38$ (dd, $J=8.2,1.5 \mathrm{~Hz}, 1 \mathrm{H}), 7.99-7.96$ (m, 1H), 7.11 (d, $J=9.4 \mathrm{~Hz}, 2 \mathrm{H}), 6.91$ (dd, $J=9.3,2.3 \mathrm{~Hz}$, 2H), $6.83(\mathrm{~d}, J=2.3 \mathrm{~Hz}, 2 \mathrm{H}) ;{ }^{13} \mathrm{C} \mathrm{NMR}\left(\mathrm{CD}_{3} \mathrm{OD}, 101 \mathrm{MHz}\right) \delta 167.7$ (C), 167.4 (C), 159.9 (C), 158.9 (C), 156.4 (C), $136.1(\mathrm{C}), 135.9(\mathrm{C}), 135.5(\mathrm{C}), 132.8(\mathrm{CH}), 132.32(\mathrm{CH}), 132.27(\mathrm{CH}), 132.0(\mathrm{CH}), 116.4(\mathrm{CH}), 114.8(\mathrm{C}), 97.8$ $(\mathrm{CH})$; Analytical HPLC: $\mathrm{t}_{\mathrm{R}}=10.7 \mathrm{~min},>99 \%$ purity $\left(10-95 \% \mathrm{MeCN} / \mathrm{H}_{2} \mathrm{O}\right.$, linear gradient, with constant $0.1 \% \mathrm{v} / \mathrm{v}$ TFA additive; $20 \mathrm{~min}$ run; $1 \mathrm{~mL} / \mathrm{min}$ flow; ESI; positive ion mode; detection at $550 \mathrm{~nm}$ ); HRMS (ESI) calcd for $\mathrm{C}_{29} \mathrm{H}_{11} \mathrm{D}_{16} \mathrm{~N}_{2} \mathrm{O}_{5}[\mathrm{M}+\mathrm{H}]^{+}$499.2919, found 499.2922.

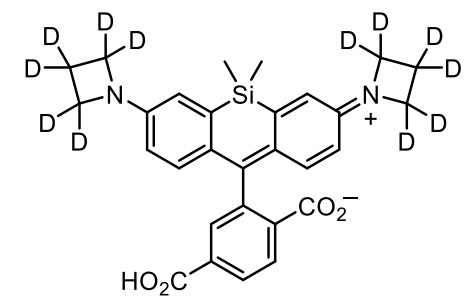

6-Carboxy-JFX $\mathbf{6 4 6}_{6}$ (S19). The title compound (98\%, green solid, TFA salt) was prepared from 6-tert-butoxycarbonyl$\mathrm{JFX}_{646}(\mathbf{S 1 6})$ according to the procedure described for $\mathbf{S 5} .{ }^{1} \mathrm{H}$ NMR $\left(\mathrm{CD}_{3} \mathrm{OD}, 400 \mathrm{MHz}\right) \delta 8.29-8.25(\mathrm{~m}, 2 \mathrm{H}), 7.80$ $(\mathrm{t}, J=1.0 \mathrm{~Hz}, 1 \mathrm{H}), 6.90(\mathrm{~d}, J=2.6 \mathrm{~Hz}, 2 \mathrm{H}), 6.87(\mathrm{~d}, J=9.2 \mathrm{~Hz}, 2 \mathrm{H}), 6.33(\mathrm{dd}, J=9.2,2.6 \mathrm{~Hz}, 2 \mathrm{H}), 0.60(\mathrm{~s}, 3 \mathrm{H}), 0.53$ $(\mathrm{s}, 3 \mathrm{H})$; Analytical HPLC: $\mathrm{t}_{\mathrm{R}}=10.9 \mathrm{~min}, 97.8 \%$ purity $\left(10-95 \% \mathrm{MeCN} / \mathrm{H}_{2} \mathrm{O}\right.$, linear gradient, with constant $0.1 \% \mathrm{v} / \mathrm{v}$ TFA additive; $20 \mathrm{~min}$ run; $1 \mathrm{~mL} / \mathrm{min}$ flow; ESI; positive ion mode; detection at $650 \mathrm{~nm}$ ); HRMS (ESI) calcd for $\mathrm{C}_{29} \mathrm{H}_{17} \mathrm{D}_{12} \mathrm{~N}_{2} \mathrm{O}_{4} \mathrm{Si}[\mathrm{M}+\mathrm{H}]^{+}$509.2644, found 509.2648.

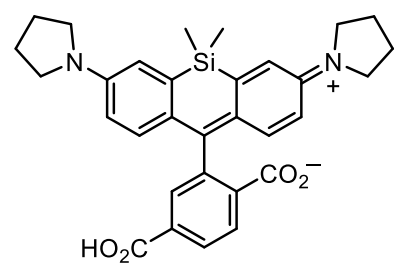

6-Carboxy-SiRhP (S20). The title compound (99\%, blue-green solid, TFA salt) was prepared from 6-tertbutoxycarbonyl-SiRhP $(\mathbf{S 1 7})$ according to the procedure described for $\mathbf{S 5} .{ }^{1} \mathrm{H} \mathrm{NMR}\left(\mathrm{CD}_{3} \mathrm{OD}, 400 \mathrm{MHz}\right) \delta 8.34(\mathrm{~d}, J$ $=8.2 \mathrm{~Hz}, 1 \mathrm{H}), 8.29(\mathrm{dd}, J=8.2,1.7 \mathrm{~Hz}, 1 \mathrm{H}), 7.82(\mathrm{~d}, J=1.6 \mathrm{~Hz}, 1 \mathrm{H}), 7.20(\mathrm{~d}, J=2.7 \mathrm{~Hz}, 2 \mathrm{H}), 6.94(\mathrm{~d}, J=9.4 \mathrm{~Hz}$, 2H), $6.61(\mathrm{dd}, J=9.5,2.7 \mathrm{~Hz}, 2 \mathrm{H}), 3.73-3.58(\mathrm{~m}, 8 \mathrm{H}), 2.16-2.05(\mathrm{~m}, 8 \mathrm{H}), 0.64(\mathrm{~s}, 3 \mathrm{H}), 0.57(\mathrm{~s}, 3 \mathrm{H}) ;{ }^{13} \mathrm{C}$ NMR $\left(\mathrm{CD}_{3} \mathrm{OD}, 101 \mathrm{MHz}\right) \delta 170.3(\mathrm{C}), 168.0(\mathrm{C}), 167.5(\mathrm{C}), 152.9$ (C), $149.3(\mathrm{C}), 142.5(\mathrm{C}), 141.6(\mathrm{CH}), 136.1(\mathrm{C}), 135.2$ $(\mathrm{C}), 132.5(\mathrm{CH}), 132.4(\mathrm{CH}), 131.0(\mathrm{CH}), 129.1(\mathrm{C}), 122.6(\mathrm{CH}), 115.7(\mathrm{CH}), 49.9\left(\mathrm{CH}_{2}\right), 26.1\left(\mathrm{CH}_{2}\right),-0.8\left(\mathrm{CH}_{3}\right),-1.8$ 
$\left(\mathrm{CH}_{3}\right)$; Analytical HPLC: $\mathrm{t}_{\mathrm{R}}=11.5 \mathrm{~min},>99 \%$ purity $\left(10-95 \% \mathrm{MeCN} / \mathrm{H}_{2} \mathrm{O}\right.$, linear gradient, with constant $0.1 \% \mathrm{v} / \mathrm{v}$ TFA additive; $20 \mathrm{~min}$ run; $1 \mathrm{~mL} / \mathrm{min}$ flow; ESI; positive ion mode; detection at $650 \mathrm{~nm}$ ); HRMS (ESI) calcd for $\mathrm{C}_{31} \mathrm{H}_{33} \mathrm{~N}_{2} \mathrm{O}_{4} \mathrm{Si}[\mathrm{M}+\mathrm{H}]^{+}$525.2204, found 525.2214.

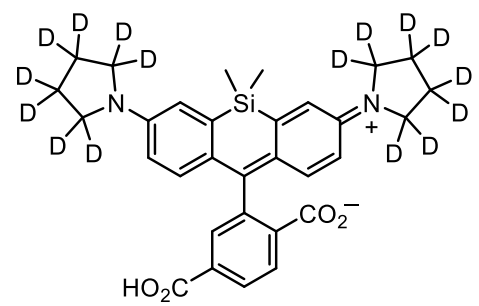

6-Carboxy-JFX650 (S21). The title compound ( 99\%, blue-green solid, TFA salt) was prepared from 6-tertbutoxycarbonyl-JFX 650 (S18) according to the procedure described for S5. ${ }^{1} \mathrm{H}$ NMR $\left(\mathrm{CD}_{3} \mathrm{OD}, 400 \mathrm{MHz}\right) \delta 8.34$ (d, $J$ $=8.1 \mathrm{~Hz}, 1 \mathrm{H}), 8.29(\mathrm{dd}, J=8.2,1.6 \mathrm{~Hz}, 1 \mathrm{H}), 7.83-7.81(\mathrm{~m}, 1 \mathrm{H}), 7.19(\mathrm{~d}, J=2.7 \mathrm{~Hz}, 2 \mathrm{H}), 6.94(\mathrm{~d}, J=9.5 \mathrm{~Hz}, 2 \mathrm{H})$, $6.60(\mathrm{dd}, J=9.5,2.7 \mathrm{~Hz}, 2 \mathrm{H}), 0.64(\mathrm{~s}, 3 \mathrm{H}), 0.57(\mathrm{~s}, 3 \mathrm{H}) ;{ }^{13} \mathrm{C}$ NMR $\left(\mathrm{CD}_{3} \mathrm{OD}, 101 \mathrm{MHz}\right) \delta 168.0(\mathrm{C}), 167.5(\mathrm{C}), 153.0$ (C), $149.3(\mathrm{C}), 142.5(\mathrm{C}), 141.6(\mathrm{CH}), 136.1(\mathrm{C}), 135.2(\mathrm{C}), 132.5(\mathrm{CH}), 132.4(\mathrm{CH}), 131.0(\mathrm{CH}), 129.0(\mathrm{C}), 122.6$ $(\mathrm{CH}), 115.7(\mathrm{CH}),-0.8\left(\mathrm{CH}_{3}\right),-1.8\left(\mathrm{CH}_{3}\right)$; Analytical HPLC: $\mathrm{t}_{\mathrm{R}}=11.5 \mathrm{~min},>99 \%$ purity $\left(10-95 \% \mathrm{MeCN} / \mathrm{H}_{2} \mathrm{O}\right.$, linear gradient, with constant $0.1 \% \mathrm{v} / \mathrm{v}$ TFA additive; $20 \mathrm{~min}$ run; $1 \mathrm{~mL} / \mathrm{min}$ flow; ESI; positive ion mode; detection at 650 nm); HRMS (ESI) calcd for $\mathrm{C}_{31} \mathrm{H}_{17} \mathrm{D}_{16} \mathrm{~N}_{2} \mathrm{O}_{4} \mathrm{Si}[\mathrm{M}+\mathrm{H}]^{+} 541.3208$, found 541.3213. 


\section{N-Hydroxysuccinimidyl Ester Formation}

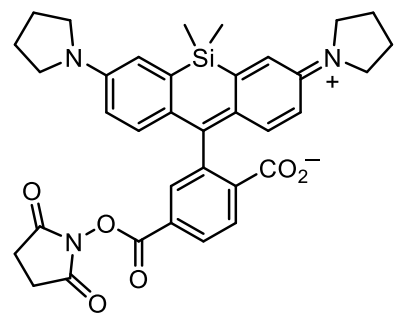

SiRhp-NHS (S22). 6-Carboxy-SiRhP (S20; $244 \mathrm{mg}, 0.382 \mathrm{mmol}$, TFA salt) was combined with DSC (235 mg, 0.917 mmol, 2.4 eq) in DMF (5 mL). After adding $\mathrm{Et}_{3} \mathrm{~N}(319 \mu \mathrm{L}, 2.29 \mathrm{mmol}, 6 \mathrm{eq})$ and DMAP (4.7 mg, $\left.38.2 \mu \mathrm{mol}, 0.1 \mathrm{eq}\right)$, the reaction was stirred at room temperature for $30 \mathrm{~min}$. It was subsequently diluted with $10 \% \mathrm{w} / \mathrm{v}$ citric acid and extracted with EtOAc $(2 \times)$. The combined organic extracts were washed with brine, dried over anhydrous $\mathrm{MgSO}_{4}$, filtered, and concentrated in vacuo. Silica gel chromatography (0-100\% EtOAc/toluene, linear gradient) yielded 206 $\mathrm{mg}(87 \%)$ of $\mathbf{S 2 2}$ as a blue-green solid. ${ }^{1} \mathrm{H}$ NMR $\left(\mathrm{CDCl}_{3}, 400 \mathrm{MHz}\right) \delta 8.27(\mathrm{dd}, J=8.0,1.4 \mathrm{~Hz}, 1 \mathrm{H}), 8.07(\mathrm{dd}, J=$ 8.0, $0.8 \mathrm{~Hz}, 1 \mathrm{H}), 8.00(\mathrm{dd}, J=1.3,0.8 \mathrm{~Hz}, 1 \mathrm{H}), 6.79(\mathrm{~d}, J=2.7 \mathrm{~Hz}, 2 \mathrm{H}), 6.74(\mathrm{~d}, J=8.8 \mathrm{~Hz}, 2 \mathrm{H}), 6.43(\mathrm{dd}, J=8.9$, $2.7 \mathrm{~Hz}, 2 \mathrm{H}), 3.35-3.25(\mathrm{~m}, 8 \mathrm{H}), 2.88(\mathrm{~s}, 4 \mathrm{H}), 2.04-1.94(\mathrm{~m}, 8 \mathrm{H}), 0.64(\mathrm{~s}, 3 \mathrm{H}), 0.58(\mathrm{~s}, 3 \mathrm{H}) ;{ }^{13} \mathrm{C} \mathrm{NMR}\left(\mathrm{CDCl}_{3}, 101\right.$ MHz) $\delta 169.6(\mathrm{C}), 168.9$ (C), $161.2(\mathrm{C}), 155.8$ (C), 147.0 (C), 136.9 (C), 132.0 (C), $130.6(\mathrm{CH}), 130.1$ (C), 129.7 (C), $128.3(\mathrm{CH}), 126.8(\mathrm{CH}), 126.2(\mathrm{CH}), 116.1(\mathrm{CH}), 113.2(\mathrm{CH}), 92.9(\mathrm{C}), 47.6\left(\mathrm{CH}_{2}\right), 25.8\left(\mathrm{CH}_{2}\right), 25.6\left(\mathrm{CH}_{2}\right), 0.4$ $\left(\mathrm{CH}_{3}\right),-1.1\left(\mathrm{CH}_{3}\right)$; Analytical HPLC: $\mathrm{t}_{\mathrm{R}}=12.2 \mathrm{~min}, 98.6 \%$ purity $\left(10-95 \% \mathrm{MeCN} / \mathrm{H}_{2} \mathrm{O}\right.$, linear gradient, with constant $0.1 \% \mathrm{v} / \mathrm{v}$ TFA additive; $20 \mathrm{~min}$ run; $1 \mathrm{~mL} / \mathrm{min}$ flow; ESI; positive ion mode; detection at $650 \mathrm{~nm}$ ); HRMS (ESI) calcd for $\mathrm{C}_{35} \mathrm{H}_{36} \mathrm{~N}_{3} \mathrm{O}_{6} \mathrm{Si}[\mathrm{M}+\mathrm{H}]^{+}$622.2368, found 622.2369 .

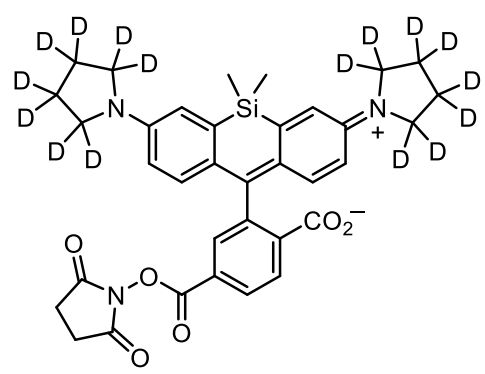

JFX $650-N H S$ (S23). The title compound (92\%, blue-green solid) was prepared from 6-carboxy-JFX650 (S21) according to the procedure described for $\mathbf{S 2 2} .{ }^{~} \mathrm{H} \mathrm{NMR}\left(\mathrm{CDCl}_{3}, 400 \mathrm{MHz}\right) \delta 8.27$ (dd, $\left.J=8.0,1.4 \mathrm{~Hz}, 1 \mathrm{H}\right), 8.07(\mathrm{dd}, J=8.0$, $0.6 \mathrm{~Hz}, 1 \mathrm{H}), 8.01(\mathrm{dd}, J=1.4,0.8 \mathrm{~Hz}, 1 \mathrm{H}), 6.78(\mathrm{~d}, J=2.8 \mathrm{~Hz}, 2 \mathrm{H}), 6.73(\mathrm{~d}, J=8.8 \mathrm{~Hz}, 2 \mathrm{H}), 6.42(\mathrm{dd}, J=8.8,2.8$ $\mathrm{Hz}, 2 \mathrm{H}), 2.89(\mathrm{~s}, 4 \mathrm{H}), 0.64(\mathrm{~s}, 3 \mathrm{H}), 0.58(\mathrm{~s}, 3 \mathrm{H})$; Analytical HPLC: $\mathrm{t}_{\mathrm{R}}=12.1 \mathrm{~min}, 98.8 \%$ purity $\left(10-95 \% \mathrm{MeCN} / \mathrm{H}_{2} \mathrm{O}\right.$, linear gradient, with constant $0.1 \% \mathrm{v} / \mathrm{v}$ TFA additive; $20 \mathrm{~min}$ run; $1 \mathrm{~mL} / \mathrm{min}$ flow; ESI; positive ion mode; detection at $650 \mathrm{~nm}$ ); HRMS (ESI) calcd for $\mathrm{C}_{35} \mathrm{H}_{20} \mathrm{D}_{16} \mathrm{~N}_{3} \mathrm{O}_{6} \mathrm{Si}[\mathrm{M}+\mathrm{H}]^{+}$638.3372, found 638.3380. 


\section{HaloTag Ligand Formation}

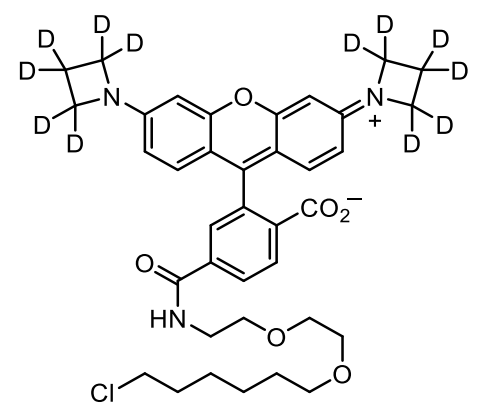

JFX $_{549}-$ HaloTag ligand (20 $)$. 6-Carboxy-JFX ${ }_{549}(\mathbf{S 5} ; 25 \mathrm{mg}, 43.1 \mu \mathrm{mol}$, TFA salt) was combined with DSC (26.5 $\mathrm{mg}, 0.103 \mathrm{mmol}, 2.4 \mathrm{eq})$ in DMF ( $1 \mathrm{~mL})$. After adding $\mathrm{Et}_{3} \mathrm{~N}(36.0 \mu \mathrm{L}, 0.258 \mathrm{mmol}, 6 \mathrm{eq})$ and DMAP $(0.5 \mathrm{mg}, 4.3$ $\mu \mathrm{mol}, 0.1 \mathrm{eq})$, the reaction was stirred at room temperature for $30 \mathrm{~min}$. A solution of 2-(2-((6chlorohexyl)oxy)ethoxy)-ethanamine (S8, 'HaloTag(O2)amine'; $43.6 \mathrm{mg}, 0.129 \mathrm{mmol}, 3 \mathrm{eq})$ in DMF (500 $\mu \mathrm{L})$ was then added. The reaction was stirred an additional $2 \mathrm{~h}$ at room temperature. Purification of the crude reaction mixture by reverse phase HPLC (20-60\% $\mathrm{MeCN} / \mathrm{H}_{2} \mathrm{O}$, linear gradient, with constant $0.1 \% \mathrm{v} / \mathrm{v}$ TFA additive) afforded 22.4 $\mathrm{mg}\left(66 \%\right.$, TFA salt) of $\mathbf{2 0}_{\mathrm{D}}$ as a dark red solid. ${ }^{1} \mathrm{H} \mathrm{NMR}\left(\mathrm{CD}_{3} \mathrm{OD}, 400 \mathrm{MHz}\right) \delta 8.78(\mathrm{t}, J=5.5 \mathrm{~Hz}, 1 \mathrm{H}), 8.39(\mathrm{~d}, J=$ $8.2 \mathrm{~Hz}, 1 \mathrm{H}), 8.20(\mathrm{dd}, J=8.2,1.8 \mathrm{~Hz}, 1 \mathrm{H}), 7.80(\mathrm{~d}, J=1.7 \mathrm{~Hz}, 1 \mathrm{H}), 7.06(\mathrm{~d}, J=9.2 \mathrm{~Hz}, 2 \mathrm{H}), 6.60(\mathrm{dd}, J=9.2,2.2$ $\mathrm{Hz}, 2 \mathrm{H}), 6.55(\mathrm{~d}, J=2.2 \mathrm{~Hz}, 2 \mathrm{H}), 3.69-3.55(\mathrm{~m}, 8 \mathrm{H}), 3.53(\mathrm{t}, J=6.6 \mathrm{~Hz}, 2 \mathrm{H}), 3.43(\mathrm{t}, J=6.5 \mathrm{~Hz}, 2 \mathrm{H}), 1.76-1.67$ $(\mathrm{m}, 2 \mathrm{H}), 1.55-1.46(\mathrm{~m}, 2 \mathrm{H}), 1.45-1.28(\mathrm{~m}, 4 \mathrm{H})$; Analytical HPLC: $\mathrm{t}_{\mathrm{R}}=12.3 \mathrm{~min},>99 \%$ purity $\left(10-95 \% \mathrm{MeCN} / \mathrm{H}_{2} \mathrm{O}\right.$, linear gradient, with constant $0.1 \% \mathrm{v} / \mathrm{v}$ TFA additive; $20 \mathrm{~min}$ run; $1 \mathrm{~mL} / \mathrm{min}$ flow; ESI; positive ion mode; detection at $550 \mathrm{~nm}$ ); HRMS (ESI) calcd for $\mathrm{C}_{37} \mathrm{H}_{31} \mathrm{D}_{12} \mathrm{ClN}_{3} \mathrm{O}_{6}[\mathrm{M}+\mathrm{H}]^{+} 672.3588$, found 672.3590 .

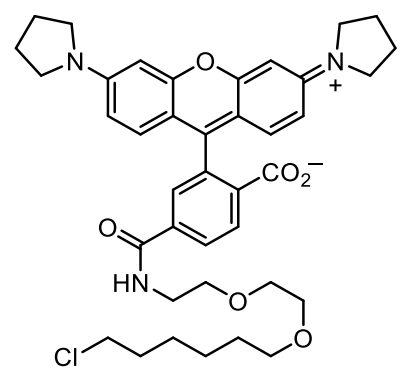

$\mathbf{R h}_{\mathrm{P}}-$ HaloTag ligand (21). The title compound (58\%, dark red-purple solid, TFA salt) was prepared from 6-carboxy$\mathrm{Rh}_{\mathrm{P}}$ (S6) and 2-(2-((6-chlorohexyl)oxy)ethoxy)ethanamine (S8) according to the procedure described for $\mathbf{2 0}_{\mathrm{D}}$. ${ }^{1} \mathrm{H}$ $\operatorname{NMR}\left(\mathrm{CD}_{3} \mathrm{OD}, 400 \mathrm{MHz}\right) \delta 8.76(\mathrm{t}, J=5.3 \mathrm{~Hz}, 1 \mathrm{H}), 8.40(\mathrm{~d}, J=8.2 \mathrm{~Hz}, 1 \mathrm{H}), 8.21(\mathrm{dd}, J=8.3,1.8 \mathrm{~Hz}, 1 \mathrm{H}), 7.83(\mathrm{~d}$, $J=1.7 \mathrm{~Hz}, 1 \mathrm{H}), 7.12(\mathrm{~d}, J=9.3 \mathrm{~Hz}, 2 \mathrm{H}), 6.91(\mathrm{dd}, J=9.3,2.3 \mathrm{~Hz}, 2 \mathrm{H}), 6.84(\mathrm{~d}, J=2.3 \mathrm{~Hz}, 2 \mathrm{H}), 3.71-3.54(\mathrm{~m}$, $16 \mathrm{H}), 3.51(\mathrm{~d}, J=6.6 \mathrm{~Hz}, 2 \mathrm{H}), 3.43(\mathrm{t}, J=6.5 \mathrm{~Hz}, 2 \mathrm{H}), 2.20-2.05(\mathrm{~m}, 8 \mathrm{H}), 1.76-1.66(\mathrm{~m}, 2 \mathrm{H}), 1.54-1.45(\mathrm{~m}, 2 \mathrm{H})$, $1.45-1.26(\mathrm{~m}, 4 \mathrm{H})$; Analytical HPLC: $\mathrm{t}_{\mathrm{R}}=13.2 \mathrm{~min}, 98.4 \%$ purity $\left(10-95 \% \mathrm{MeCN} / \mathrm{H}_{2} \mathrm{O}\right.$, linear gradient, with constant $0.1 \% \mathrm{v} / \mathrm{v}$ TFA additive; $20 \mathrm{~min}$ run; $1 \mathrm{~mL} / \mathrm{min}$ flow; ESI; positive ion mode; detection at $550 \mathrm{~nm}$ ); HRMS (ESI) calcd for $\mathrm{C}_{39} \mathrm{H}_{47} \mathrm{ClN}_{3} \mathrm{O}_{6}[\mathrm{M}+\mathrm{H}]^{+} 688.3148$, found 688.3156 . 


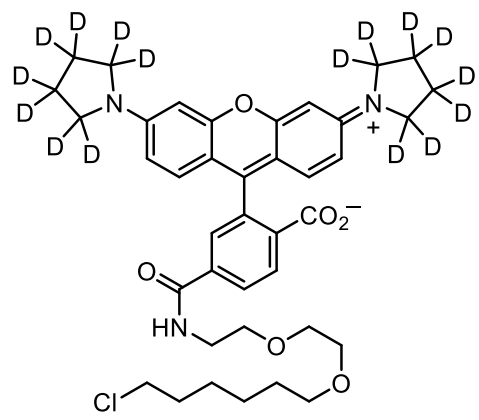

JFX $_{554}-$ HaloTag ligand (21 $\left.\mathbf{2}_{\mathrm{D}}\right)$. The title compound (63\%, dark red-purple solid, TFA salt) was prepared from 6carboxy-JFX $_{554}$ (S7) and 2-(2-((6-chlorohexyl)oxy)ethoxy)ethanamine (S8) according to the procedure described for 20. ${ }^{~}{ }^{\mathrm{H}} \mathrm{NMR}\left(\mathrm{CD}_{3} \mathrm{OD}, 400 \mathrm{MHz}\right) \delta 8.76(\mathrm{t}, J=5.2 \mathrm{~Hz}, 1 \mathrm{H}), 8.40(\mathrm{~d}, J=8.2 \mathrm{~Hz}, 1 \mathrm{H}), 8.21(\mathrm{dd}, J=8.3,1.8 \mathrm{~Hz}, 1 \mathrm{H})$, $7.83(\mathrm{~d}, J=1.7 \mathrm{~Hz}, 1 \mathrm{H}), 7.12(\mathrm{~d}, J=9.3 \mathrm{~Hz}, 2 \mathrm{H}), 6.91(\mathrm{dd}, J=9.3,2.3 \mathrm{~Hz}, 2 \mathrm{H}), 6.84(\mathrm{~d}, J=2.3 \mathrm{~Hz}, 2 \mathrm{H}), 3.70-3.54$ $(\mathrm{m}, 8 \mathrm{H}), 3.52(\mathrm{t}, J=6.6 \mathrm{~Hz}, 2 \mathrm{H}), 3.43(\mathrm{t}, J=6.5 \mathrm{~Hz}, 2 \mathrm{H}), 1.76-1.66(\mathrm{~m}, 2 \mathrm{H}), 1.55-1.45(\mathrm{~m}, 2 \mathrm{H}), 1.45-1.27(\mathrm{~m}$, $4 \mathrm{H})$; Analytical HPLC: $\mathrm{t}_{\mathrm{R}}=13.1 \mathrm{~min}, 98.4 \%$ purity $\left(10-95 \% \mathrm{MeCN} / \mathrm{H}_{2} \mathrm{O}\right.$, linear gradient, with constant $0.1 \% \mathrm{v} / \mathrm{v}$ TFA additive; $20 \mathrm{~min}$ run; $1 \mathrm{~mL} / \mathrm{min}$ flow; ESI; positive ion mode; detection at $550 \mathrm{~nm}$ ); HRMS (ESI) calcd for $\mathrm{C}_{39} \mathrm{H}_{31} \mathrm{D}_{16} \mathrm{ClN}_{3} \mathrm{O}_{6}[\mathrm{M}+\mathrm{H}]^{+}$704.4152, found 704.4155.

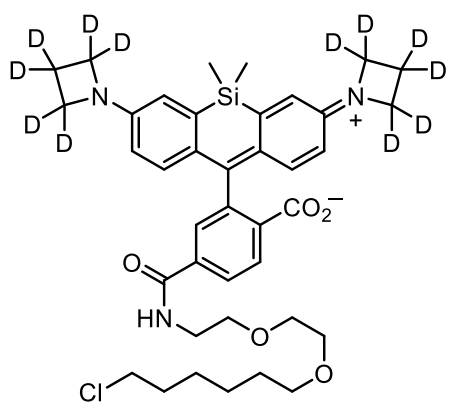

JFX $_{646}-$ HaloTag ligand (30 $\left.\mathbf{D}_{\mathrm{D}}\right)$. The title compound (79\%, pale blue-green solid) was prepared from 6-carboxy-JFX 646 (S19) and 2-(2-((6-chlorohexyl)oxy)ethoxy)ethanamine (S8) according to the procedure described for 20 ${ }_{\mathbf{D}}$. ${ }^{1} \mathrm{H}$ NMR $\left(\mathrm{CDCl}_{3}, 400 \mathrm{MHz}\right) \delta 7.98(\mathrm{~d}, J=7.9 \mathrm{~Hz}, 1 \mathrm{H}), 7.90(\mathrm{dd}, J=8.0,1.4 \mathrm{~Hz}, 1 \mathrm{H}), 7.70-7.66(\mathrm{~m}, 1 \mathrm{H}), 6.76(\mathrm{~s}, 1 \mathrm{H}), 6.75$ $(\mathrm{d}, J=8.7 \mathrm{~Hz}, 2 \mathrm{H}), 6.66(\mathrm{~d}, J=2.7 \mathrm{~Hz}, 2 \mathrm{H}), 6.26(\mathrm{dd}, J=8.6,2.7 \mathrm{~Hz}, 2 \mathrm{H}), 3.67-3.59(\mathrm{~m}, 6 \mathrm{H}), 3.56-3.53(\mathrm{~m}, 2 \mathrm{H})$, $3.50(\mathrm{t}, J=6.6 \mathrm{~Hz}, 2 \mathrm{H}), 3.39(\mathrm{t}, J=6.7 \mathrm{~Hz}, 2 \mathrm{H}), 1.76-1.69(\mathrm{~m}, 2 \mathrm{H}), 1.54-1.48(\mathrm{~m}, 2 \mathrm{H}), 1.44-1.26(\mathrm{~m}, 4 \mathrm{H}), 0.63$ $(\mathrm{s}, 3 \mathrm{H}), 0.57(\mathrm{~s}, 3 \mathrm{H})$; Analytical HPLC: $\mathrm{t}_{\mathrm{R}}=13.2 \mathrm{~min}, 98.7 \%$ purity $\left(10-95 \% \mathrm{MeCN} / \mathrm{H}_{2} \mathrm{O}\right.$, linear gradient, with constant $0.1 \% \mathrm{v} / \mathrm{v}$ TFA additive; $20 \mathrm{~min}$ run; $1 \mathrm{~mL} / \mathrm{min}$ flow; ESI; positive ion mode; detection at $650 \mathrm{~nm}$ ); HRMS (ESI) calcd for $\mathrm{C}_{39} \mathrm{H}_{37} \mathrm{D}_{12} \mathrm{ClN}_{3} \mathrm{O}_{5} \mathrm{Si}[\mathrm{M}+\mathrm{H}]^{+}$714.3878, found 714.3885. 


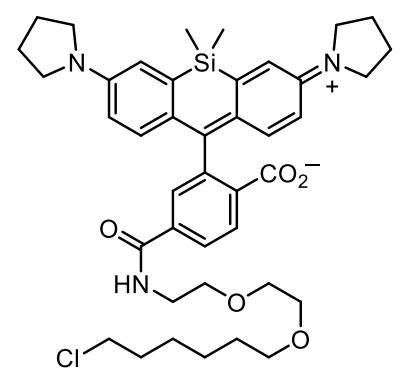

$\mathbf{S i R h}_{\mathrm{P}}$-HaloTag ligand $\quad \mathbf{( 3 1 )}$. $\left.\quad \mathrm{SiRh}_{\mathrm{P}}-\mathrm{NHS} \quad \mathbf{( S 2 2} ; \quad 75 \quad \mathrm{mg}, \quad 0.121 \quad \mathrm{mmol}\right) \quad$ and $2-(2-((6-$ chlorohexyl)oxy)ethoxy)ethanamine (S8; $61.1 \mathrm{mg}, 0.181 \mathrm{mmol}, 1.5 \mathrm{eq})$ were combined in DMF (3 mL), and DIEA $(63.0 \mu \mathrm{L}, 0.362 \mathrm{mmol}, 3 \mathrm{eq})$ was added. After stirring the reaction at room temperature for $1 \mathrm{~h}$, it was diluted with saturated $\mathrm{NaHCO}_{3}$ and extracted with EtOAc (2x). The combined organic extracts were washed with water and brine, dried over anhydrous $\mathrm{MgSO}_{4}$, filtered, and evaporated. Purification of the crude product by silica gel chromatography (10-100\% EtOAc/toluene, linear gradient) provided 31 as a pale blue-green solid $(64.4 \mathrm{mg}, 73 \%) .{ }^{1} \mathrm{H} \mathrm{NMR}\left(\mathrm{CDCl}_{3}\right.$, $400 \mathrm{MHz}) \delta 7.99(\mathrm{~d}, J=7.9 \mathrm{~Hz}, 1 \mathrm{H}), 7.91(\mathrm{dd}, J=7.9,1.4 \mathrm{~Hz}, 1 \mathrm{H}), 7.67-7.64(\mathrm{~m}, 1 \mathrm{H}), 6.79(\mathrm{~d}, J=2.7 \mathrm{~Hz}, 2 \mathrm{H})$, $6.75(\mathrm{~s}, 1 \mathrm{H}), 6.75(\mathrm{~d}, J=8.8 \mathrm{~Hz}, 2 \mathrm{H}), 6.39(\mathrm{dd}, J=8.9,2.7 \mathrm{~Hz}, 2 \mathrm{H}), 3.67-3.58(\mathrm{~m}, 6 \mathrm{H}), 3.57-3.52(\mathrm{~m}, 2 \mathrm{H}), 3.50$ $(\mathrm{t}, J=6.7 \mathrm{~Hz}, 2 \mathrm{H}), 3.39(\mathrm{t}, J=6.7 \mathrm{~Hz}, 2 \mathrm{H}), 3.35-3.24(\mathrm{~m}, 8 \mathrm{H}), 2.05-1.93(\mathrm{~m}, 8 \mathrm{H}), 1.77-1.68(\mathrm{~m}, 2 \mathrm{H}), 1.55-1.47$ $(\mathrm{m}, 2 \mathrm{H}), 1.44-1.26(\mathrm{~m}, 4 \mathrm{H}), 0.65(\mathrm{~s}, 3 \mathrm{H}), 0.59(\mathrm{~s}, 3 \mathrm{H})$; Analytical HPLC: $\mathrm{t}_{\mathrm{R}}=13.5 \mathrm{~min},>99 \%$ purity $(10-95 \%$ $\mathrm{MeCN} / \mathrm{H}_{2} \mathrm{O}$, linear gradient, with constant $0.1 \% \mathrm{v} / \mathrm{v}$ TFA additive; $20 \mathrm{~min}$ run; $1 \mathrm{~mL} / \mathrm{min}$ flow; ESI; positive ion mode; detection at $650 \mathrm{~nm}$ ); HRMS (ESI) calcd for $\mathrm{C}_{41} \mathrm{H}_{53} \mathrm{ClN}_{3} \mathrm{O}_{5} \mathrm{Si}[\mathrm{M}+\mathrm{H}]^{+} 730.3438$, found 730.3442 .

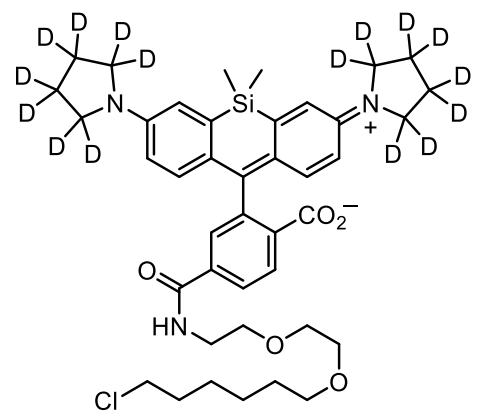

JFX $_{650}-$ HaloTag ligand (31) $)$. The title compound (77\%, pale blue-green solid) was prepared from JFX $650-\mathrm{NHS}_{\text {(S23) }}$ and 2-(2-((6-chlorohexyl)oxy)ethoxy)ethanamine (S8) according to the procedure described for 31. ${ }^{1} \mathrm{H} \mathrm{NMR}\left(\mathrm{CDCl}_{3}\right.$, $400 \mathrm{MHz}) \delta 7.99(\mathrm{dd}, J=8.0,0.5 \mathrm{~Hz}, 1 \mathrm{H}), 7.91(\mathrm{dd}, J=8.0,1.4 \mathrm{~Hz}, 1 \mathrm{H}), 7.67-7.63(\mathrm{~m}, 1 \mathrm{H}), 6.78(\mathrm{~d}, J=2.8 \mathrm{~Hz}$, $2 \mathrm{H}), 6.747(\mathrm{~d}, J=8.8 \mathrm{~Hz}, 2 \mathrm{H}), 6.744(\mathrm{~s}, 1 \mathrm{H}), 6.39(\mathrm{dd}, J=8.8,2.8 \mathrm{~Hz}, 2 \mathrm{H}), 3.66-3.58(\mathrm{~m}, 6 \mathrm{H}), 3.57-3.52(\mathrm{~m}, 2 \mathrm{H})$, $3.50(\mathrm{t}, J=6.7 \mathrm{~Hz}, 2 \mathrm{H}), 3.39(\mathrm{t}, J=6.7 \mathrm{~Hz}, 2 \mathrm{H}), 1.77-1.68(\mathrm{~m}, 2 \mathrm{H}), 1.55-1.47(\mathrm{~m}, 2 \mathrm{H}), 1.44-1.35(\mathrm{~m}, 2 \mathrm{H}), 1.34$ $-1.25(\mathrm{~m}, 2 \mathrm{H}), 0.65(\mathrm{~s}, 3 \mathrm{H}), 0.58(\mathrm{~s}, 3 \mathrm{H})$; Analytical HPLC: $\mathrm{t}_{\mathrm{R}}=13.4 \mathrm{~min},>99 \%$ purity $\left(10-95 \% \mathrm{MeCN} / \mathrm{H}_{2} \mathrm{O}\right.$, linear gradient, with constant $0.1 \%$ v/v TFA additive; $20 \mathrm{~min}$ run; $1 \mathrm{~mL} / \mathrm{min}$ flow; ESI; positive ion mode; detection at 650 $\mathrm{nm}$ ); HRMS (ESI) calcd for $\mathrm{C}_{41} \mathrm{H}_{37} \mathrm{D}_{16} \mathrm{ClN}_{3} \mathrm{O}_{5} \mathrm{Si}[\mathrm{M}+\mathrm{H}]^{+} 746.4442$, found 746.4449 . 


\section{REFERENCES}

1. Grimm, J. B.; Lavis, L. D., Synthesis of rhodamines from fluoresceins using Pd-catalyzed C-N cross-coupling. Org. Lett. 2011, 13, 6354-6357.

2. Grimm, J. B.; Sung, A. J.; Legant, W. R.; Hulamm, P.; Matlosz, S. M.; Betzig, E.; Lavis, L. D., Carbofluoresceins and carborhodamines as scaffolds for high-contrast fluorogenic probes. ACS Chem. Biol. 2013, 8, 1303-1310.

3. Grimm, J. B.; English, B. P.; Chen, J.; Slaughter, J. P.; Zhang, Z.; Revyakin, A.; Patel, R.; Macklin, J. J.; Normanno, D.; Singer, R. H.; Lionnet, T.; Lavis, L. D., A general method to improve fluorophores for live-cell and single-molecule microscopy. Nat. Methods 2015, 12, 244-250.

4. Grimm, J. B.; Brown, T. A.; Tkachuk, A. N.; Lavis, L. D., General synthetic method for Si-fluoresceins and Sirhodamines. ACS Cent. Sci. 2017, 3, 975-985.

5. Suzuki, K.; Kobayashi, A.; Kaneko, S.; Takehira, K.; Yoshihara, T.; Ishida, H.; Shiina, Y.; Oishi, S.; Tobita, S., Reevaluation of absolute luminescence quantum yields of standard solutions using a spectrometer with an integrating sphere and a back-thinned CCD detector. Phys. Chem. Chem. Phys. 2009, 11, 9850-9860.

6. Hinckley, D. A.; Seybold, P. G., A spectroscopic/thermodynamic study of the rhodamine B lactone $\rightleftharpoons$ zwitterion equilibrium. Spectrochim. Acta, Part A 1988, 44, 1053-1059.

7. Grimm, J. B.; Muthusamy, A. K.; Liang, Y.; Brown, T. A.; Lemon, W. C.; Patel, R.; Lu, R.; Macklin, J. J.; Keller, P. J.; Ji, N.; Lavis, L. D., A general method to fine-tune fluorophores for live-cell and in vivo imaging. Nat. Methods 2017, 14, 987-994.

8. Schindelin, J.; Arganda-Carreras, I.; Frise, E.; Kaynig, V.; Longair, M.; Pietzsch, T.; Preibisch, S.; Rueden, C.; Saalfeld, S.; Schmid, B.; Tinevez, J. Y.; White, D. J.; Hartenstein, V.; Eliceiri, K.; Tomancak, P.; Cardona, A., Fiji: An open-source platform for biological-image analysis. Nat. Methods 2012, 9, 676-682.

9. Grimm, J. B.; English, B. P.; Choi, H.; Muthusamy, A. K.; Mehl, B. P.; Dong, P.; Brown, T. A.; LippincottSchwartz, J.; Liu, Z.; Lionnet, T.; Lavis, L. D., Bright photoactivatable fluorophores for single-molecule imaging. Nat. Methods 2016, 13, 985-988.

10. Xie, L.; Torigoe, S. E.; Xiao, J.; Mai, D. H.; Li, L.; Davis, F. P.; Dong, P.; Marie-Nelly, H.; Grimm, J.; Lavis, L.; Darzacq, X.; Cattoglio, C.; Liu, Z.; Tjian, R., A dynamic interplay of enhancer elements regulates Klf4 expression in naïve pluripotency. Genes Dev. 2017, 31, 1795-1808.

11. Chen, J.; Zhang, Z.; Li, L.; Chen, B.-C.; Revyakin, A.; Hajj, B.; Legant, W.; Dahan, M.; Lionnet, T.; Betzig, E.; Tjian, R.; Liu, Z., Single-molecule dynamics of enhanceosome assembly in embryonic stem cells. Cell 2014, 156, 1274-1285.

12. Sergé, A.; Bertaux, N.; Rigneault, H.; Marguet, D., Dynamic multiple-target tracing to probe spatiotemporal cartography of cell membranes. Nat. Methods 2008, 5, 687-694.

13. Kövér, J.; Antus, S., Facile deoxygenation of hydroxylated flavonoids by palladium-catalysed reduction of its triflate derivatives. Z. Naturforsch. B 2005, 60, 792-796.

14. Helal, C. J.; Chappie, T. A.; Humphrey, J. M. Pyrazolo[3,4-d]pyrimidine compounds and their use as pde2 inhibitors and/or cyp3a4 inhibitors. Int. Pat. Appl. WO 2012/168817 A1, Dec 13, 2012, 2012. 

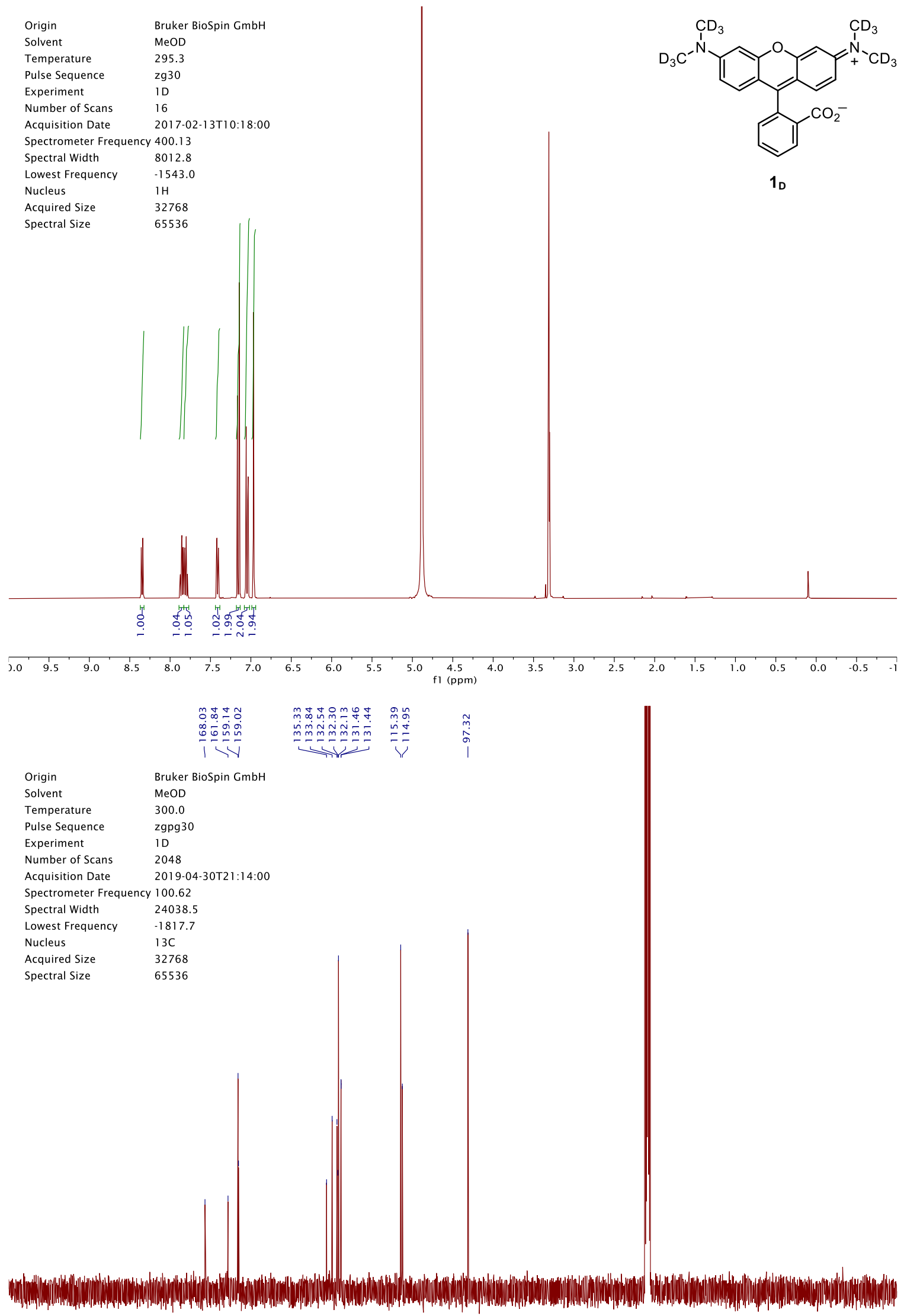

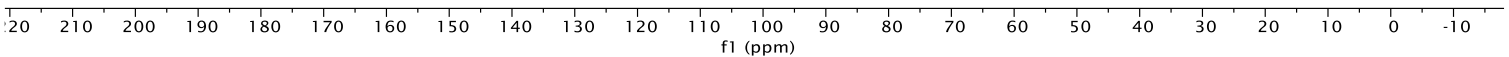




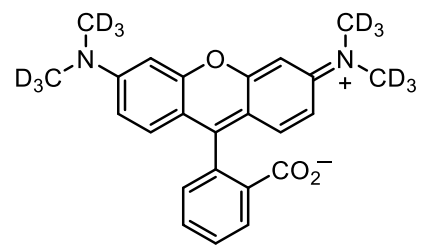

1D

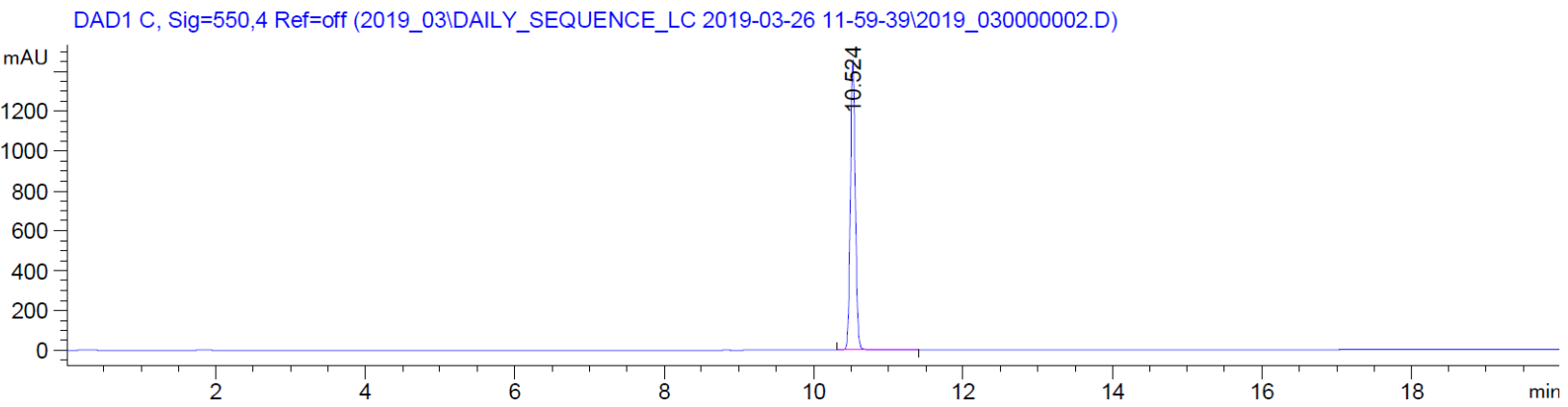

${ }^{*}$ MSD2 SPC, time=10.535:10.607 of C:ICHEM32I1IDATAI2019_03IDAILY_SEQUENCE_LC 2019-03-26 11-59-3912019_0300000002.D ES-

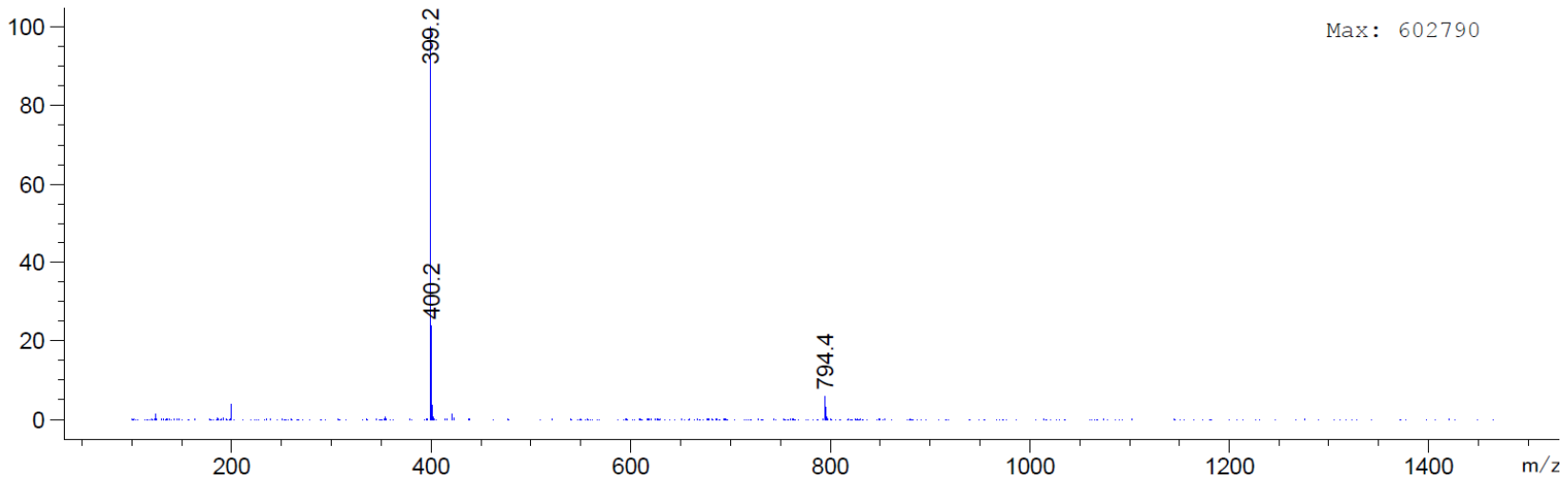



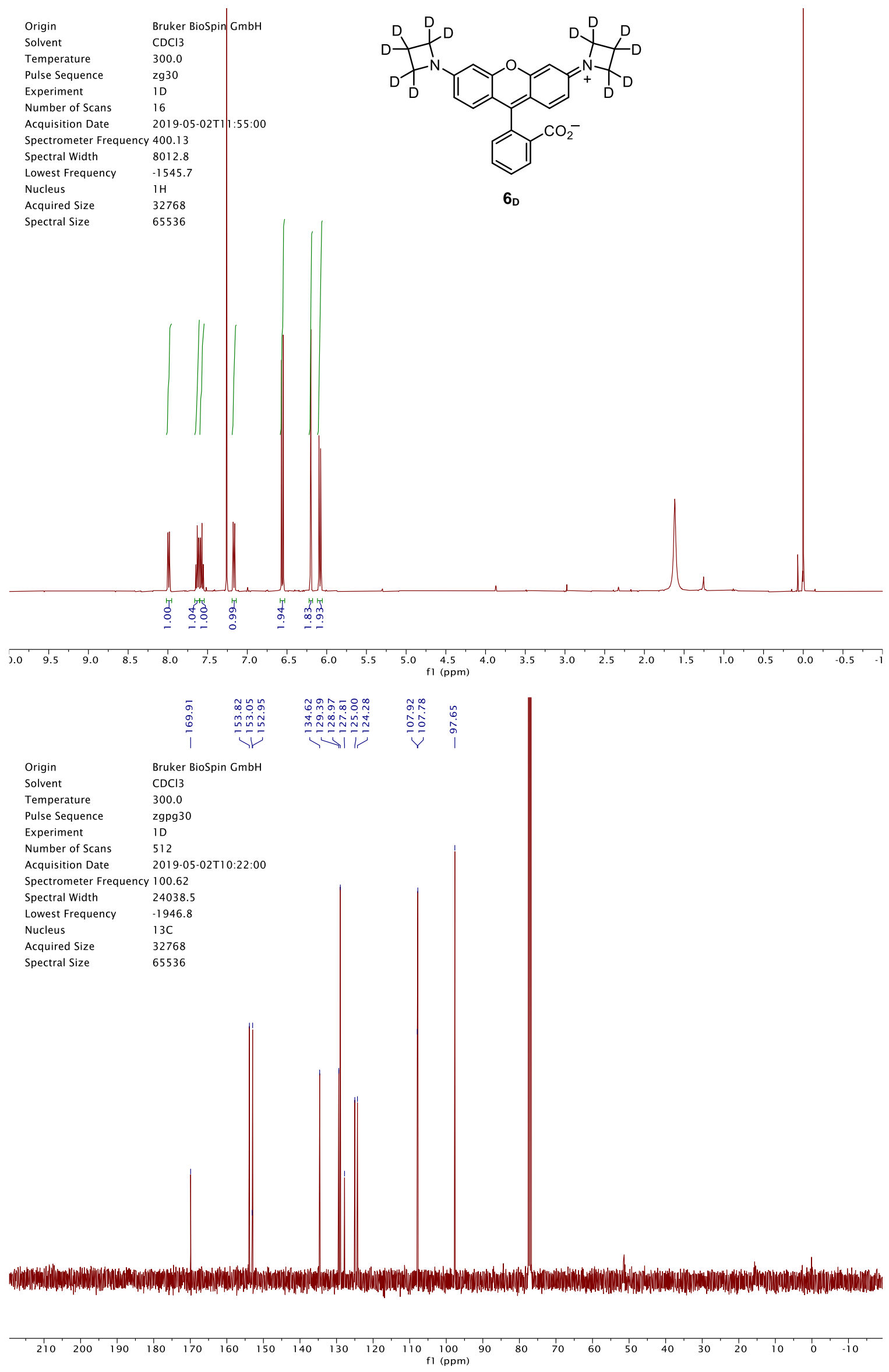


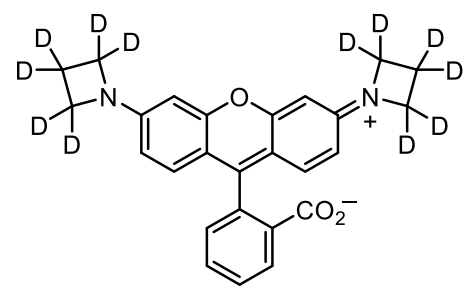

6D

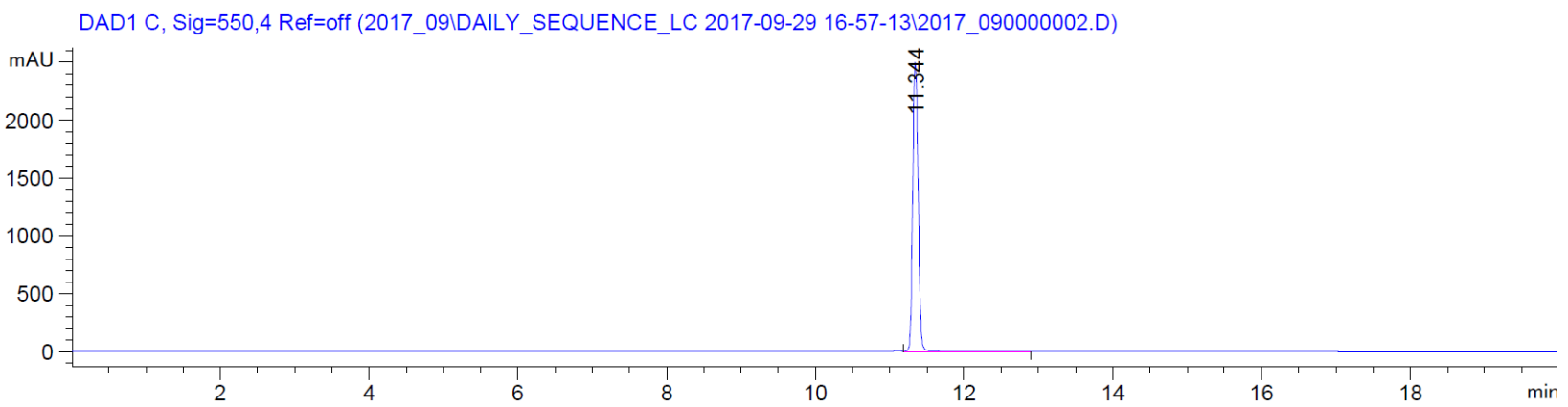

*MSD2 SPC, time=11.334:11.407 of C:ICHEM32I1IDATAI2017_09IDAILY_SEQUENCE_LC 2017-09-29 16-57-1312017_0900000002.D ES-

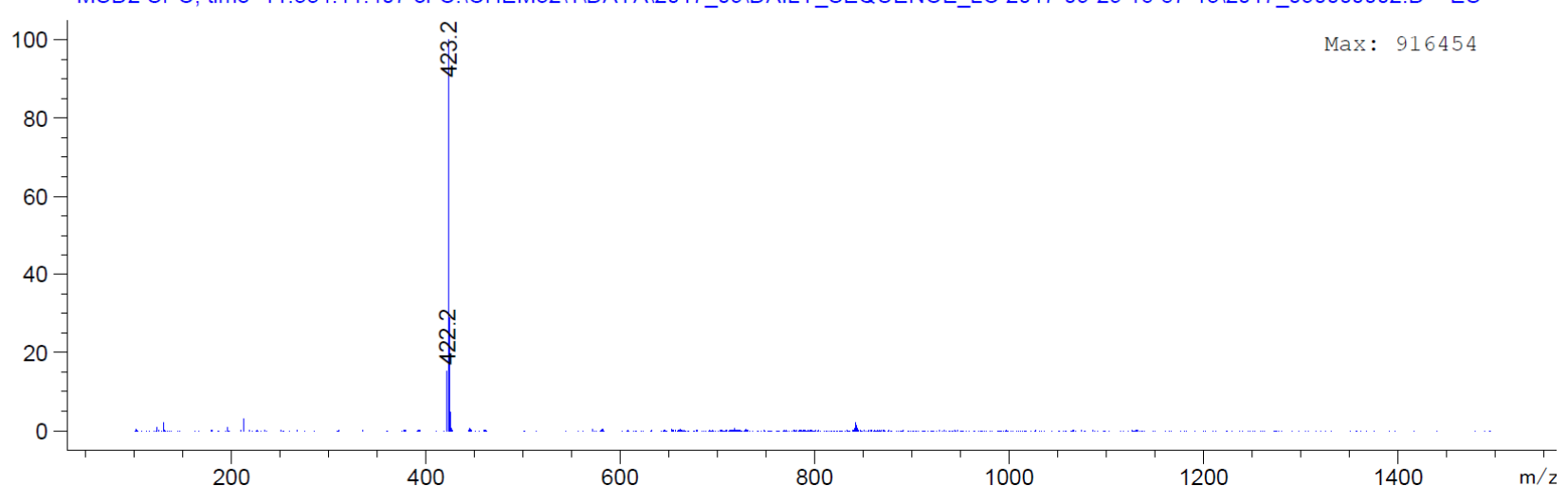



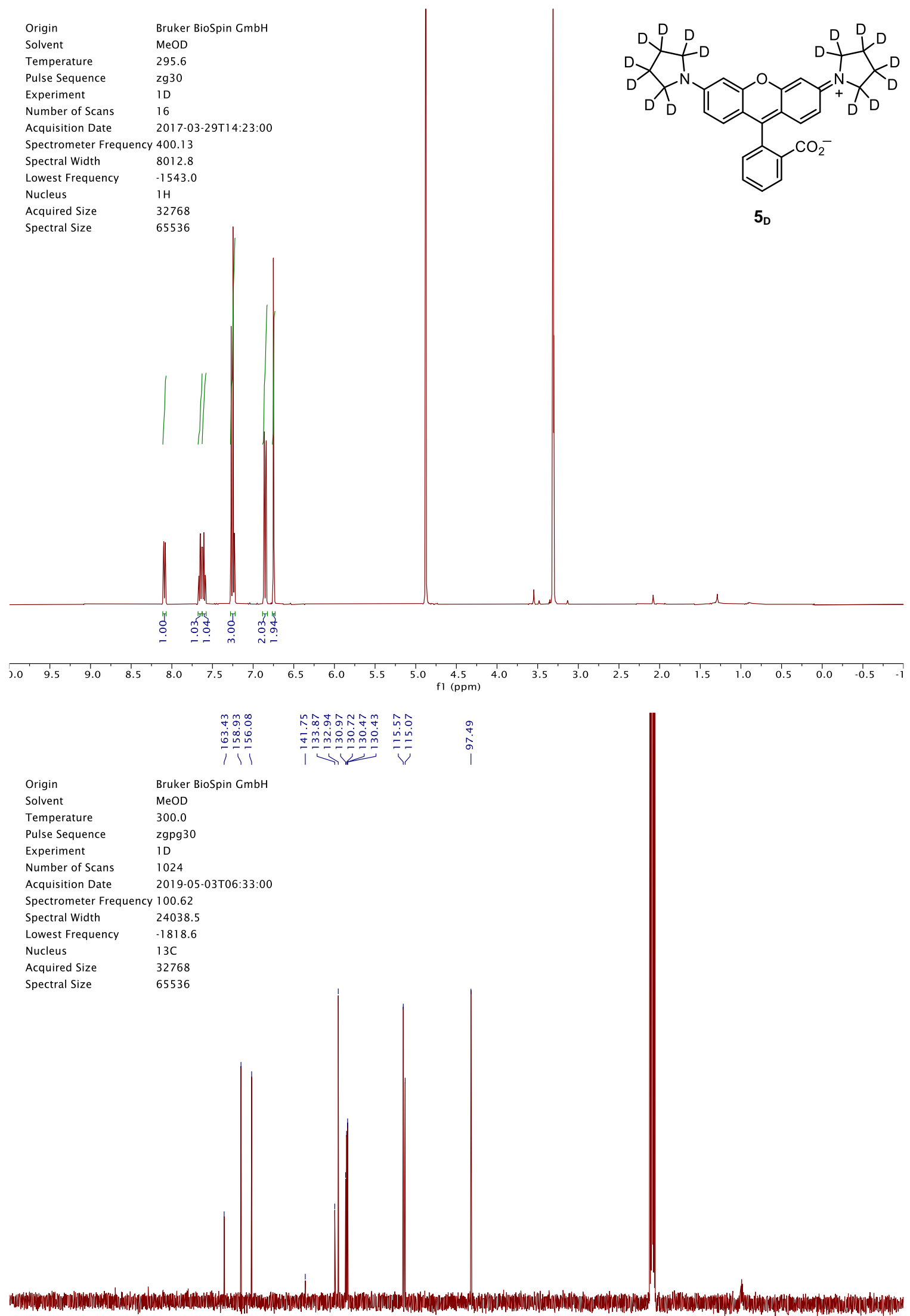

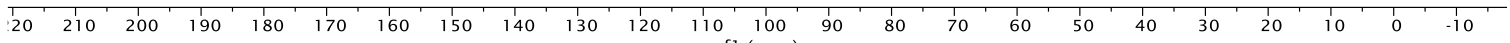




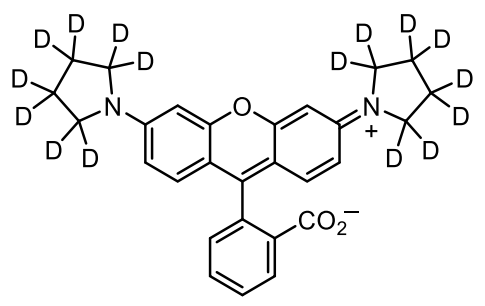

5D

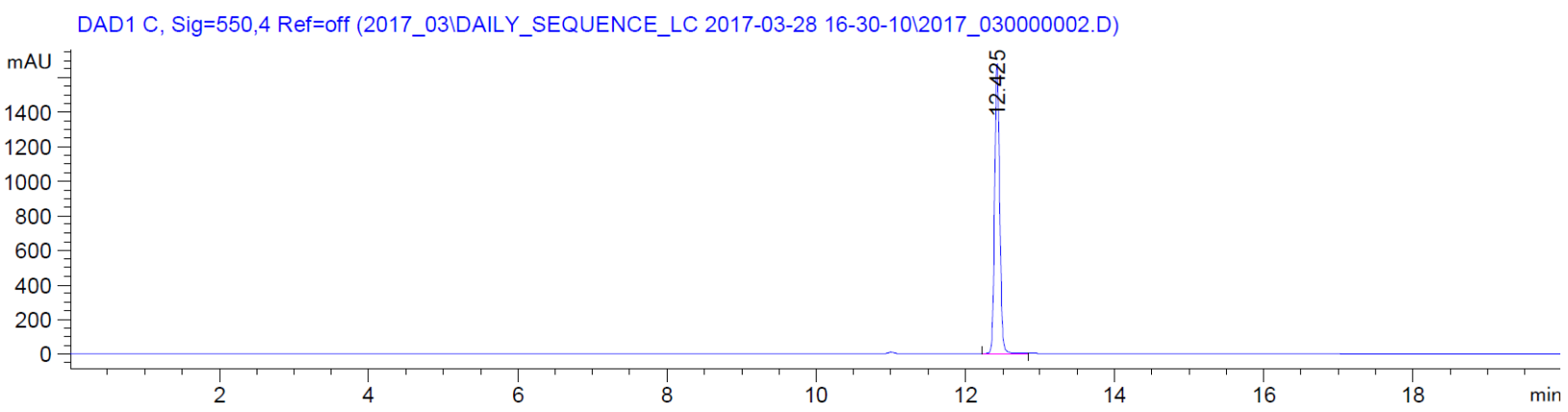

*MSD2 SPC, time=12.424:12.497 of C:ICHEM3211IDATAI2017_03IDAILY_SEQUENCE_LC 2017-03-28 16-30-1012017_0300000002.D ES-

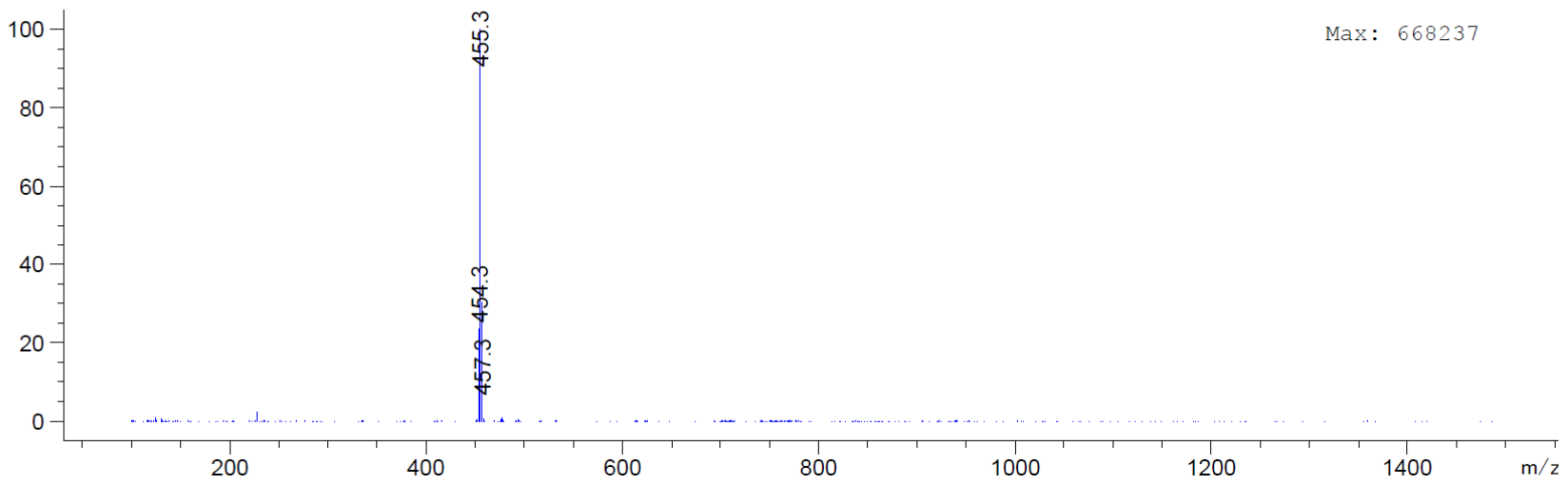



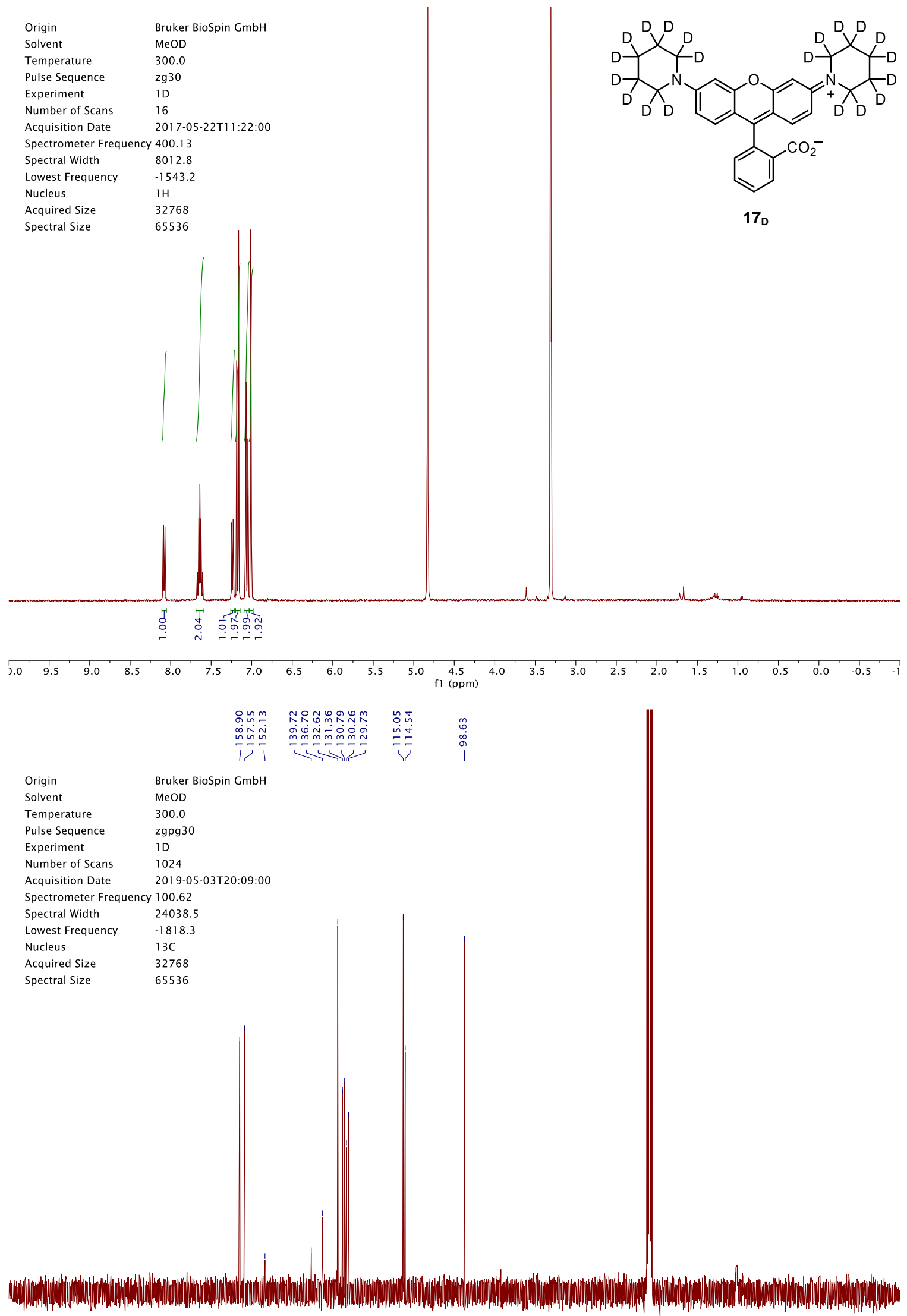

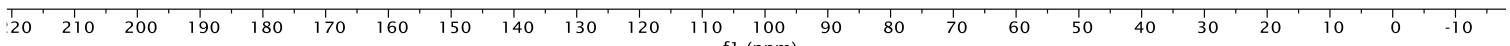




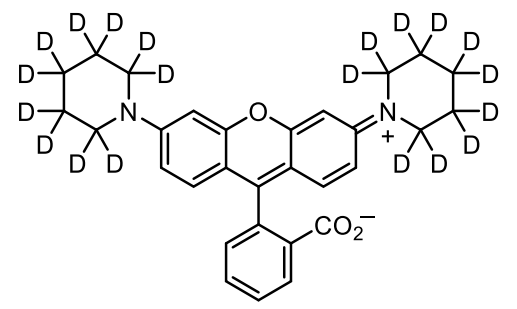

17D

DAD1 C, Sig=550,4 Ref=off (2019_03IDAILY_SEQUENCE_LC 2019-03-28 16-19-5112019_030000003.D)

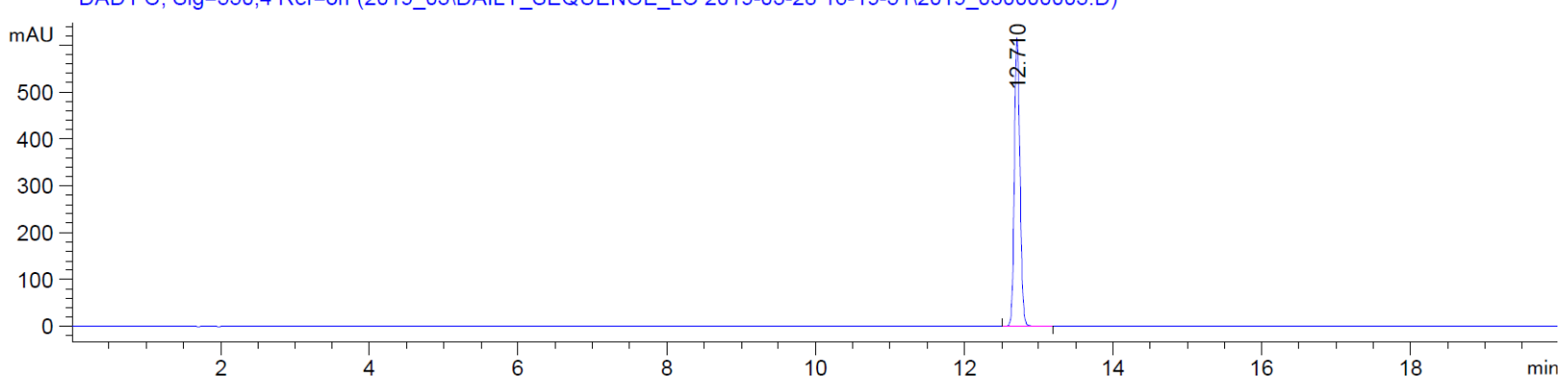

${ }^{*}$ MSD2 SPC, time=12.715:12.806 of C:ICHEM32I1IDATAI2019_03IDAILY_SEQUENCE_LC 2019-03-28 16-19-51|2019_030000003.D ES-

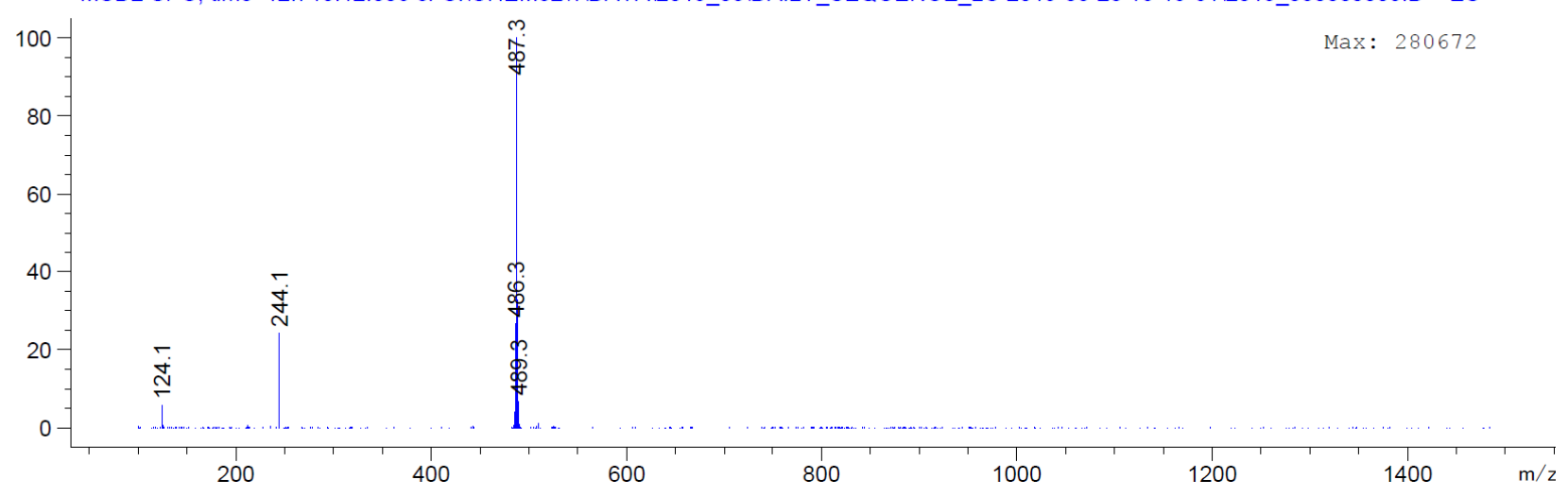




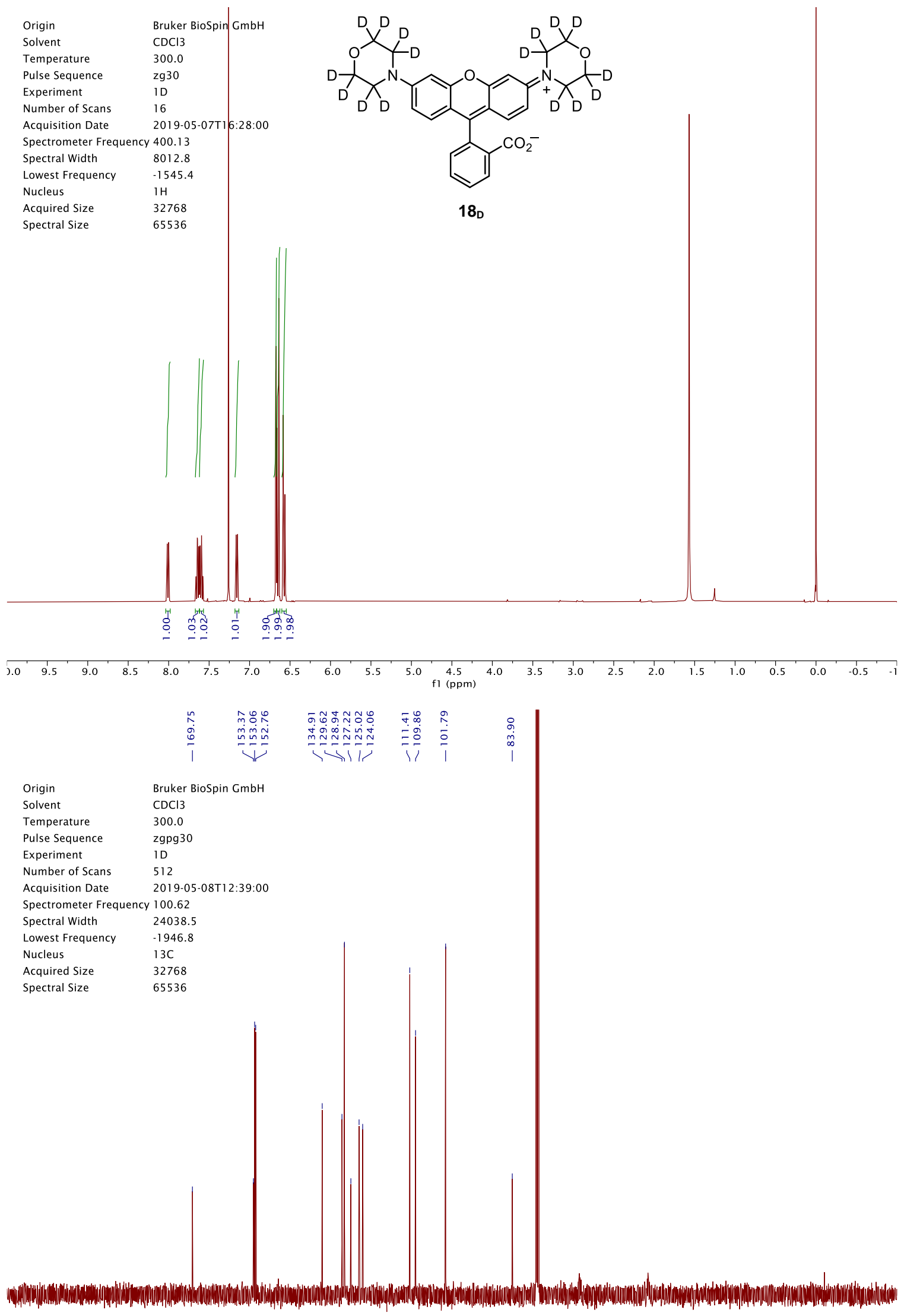

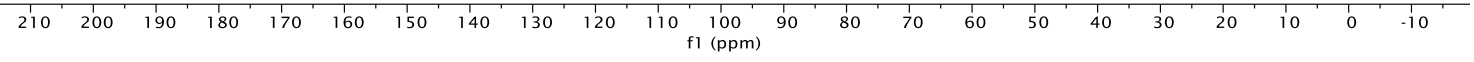




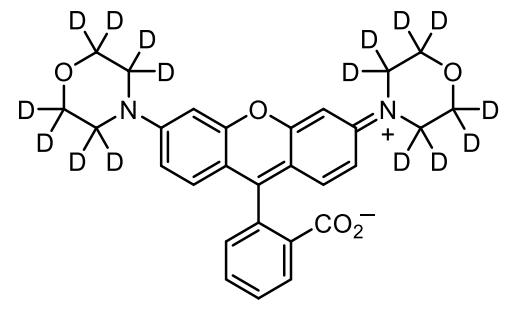

18D

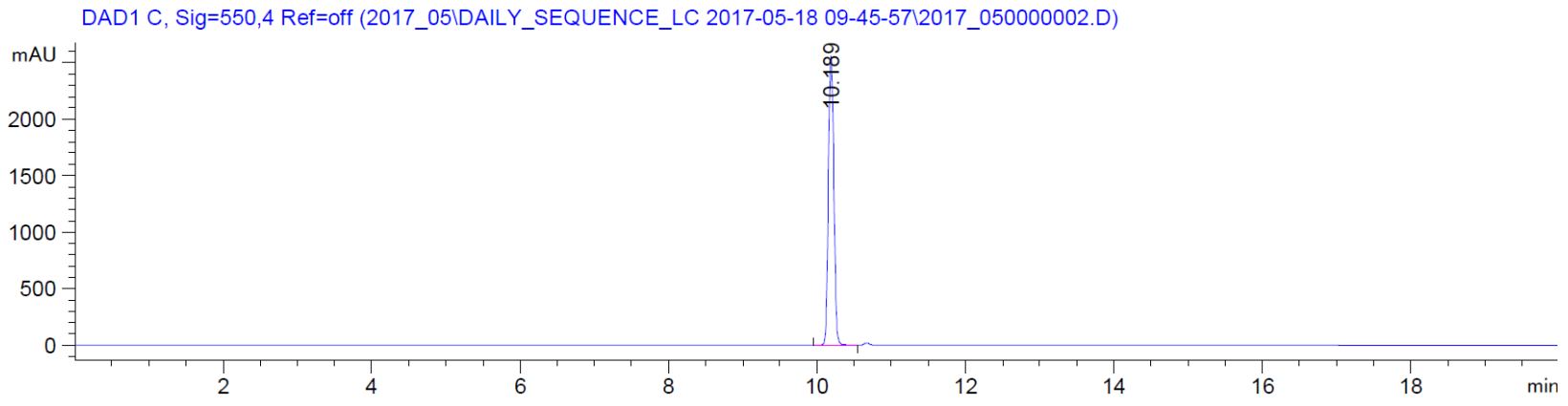

*MSD2 SPC, time=10.189:10.262 of C:ICHEM32I1IDATAI2017_05IDAILY_SEQUENCE_LC 2017-05-18 09-45-57|2017_050000002.D ES-

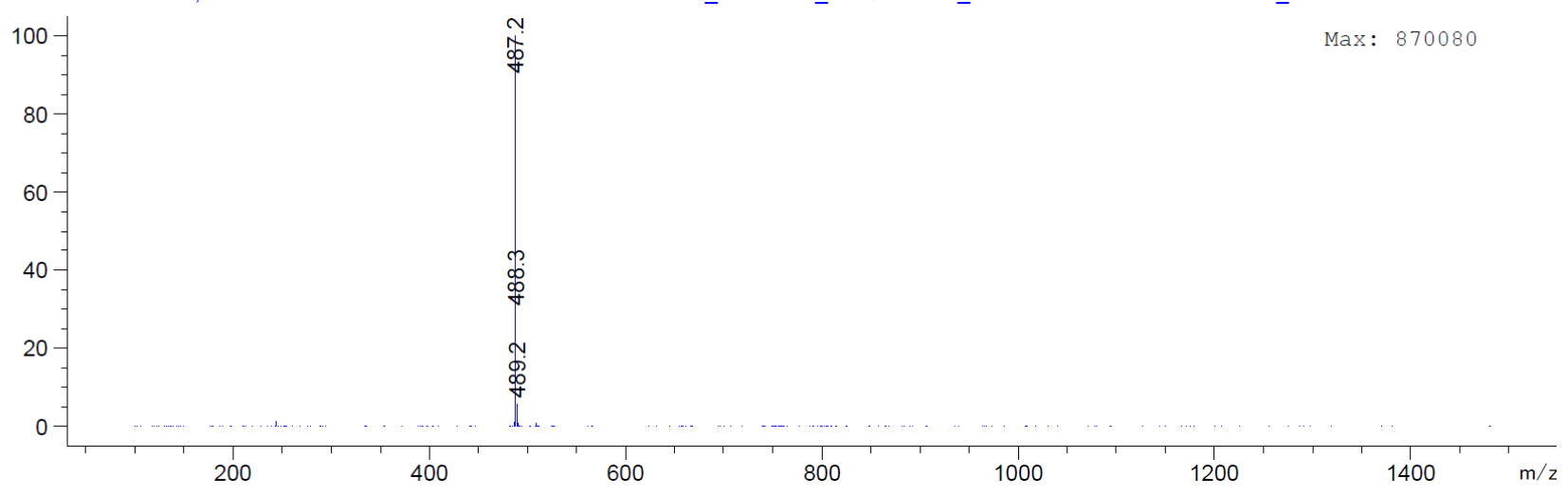



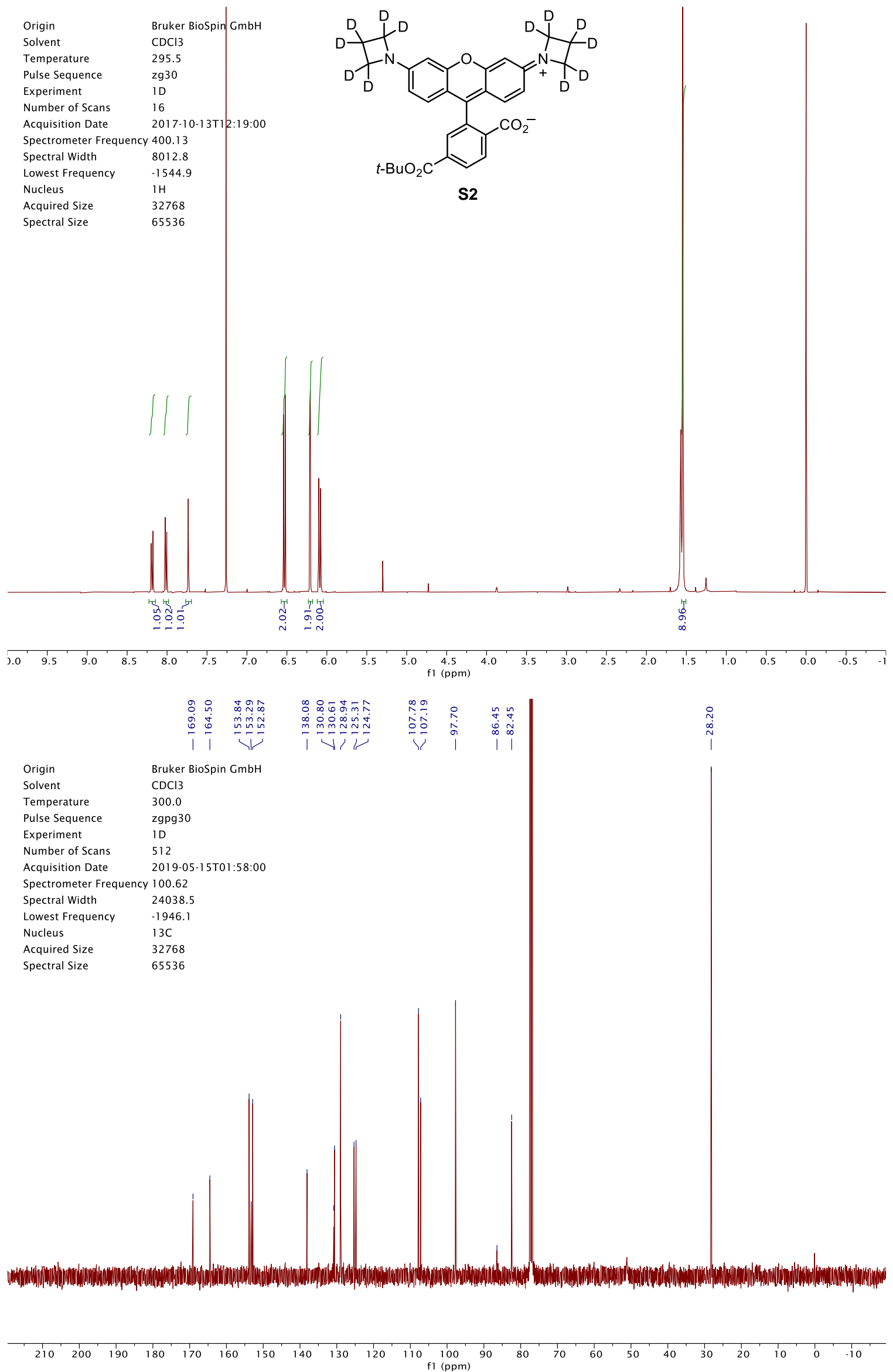


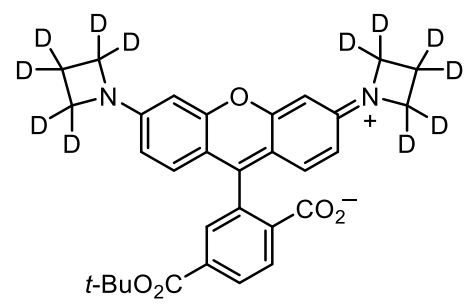

S2

DAD1 C, Sig=550,4 Ref=off (2019_03IDAILY_SEQUENCE_LC 2019-03-28 16-19-5112019_030000005.D)

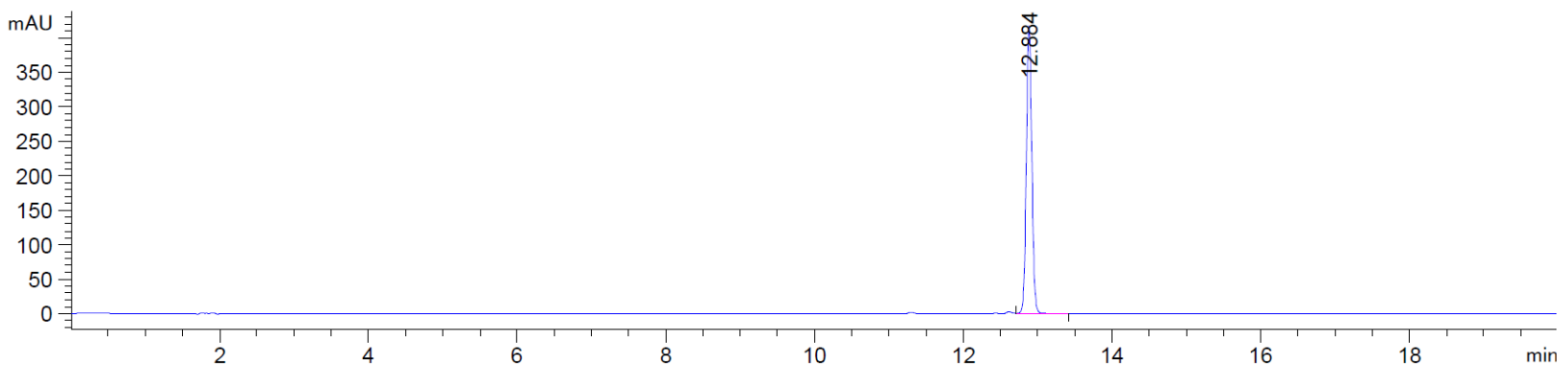

${ }^{*}$ MSD2 SPC, time=12.878:12.987 of C:ICHEM32I1IDATAI2019_03IDAILY_SEQUENCE_LC 2019-03-28 16-19-5112019_0300000005.D ES-

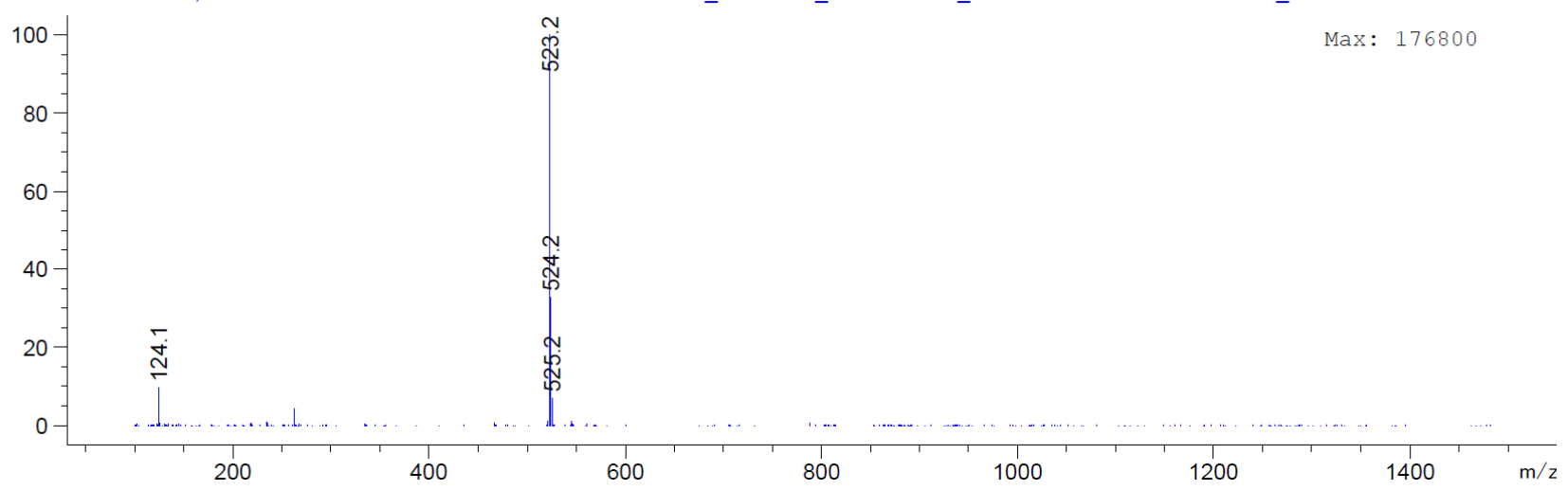




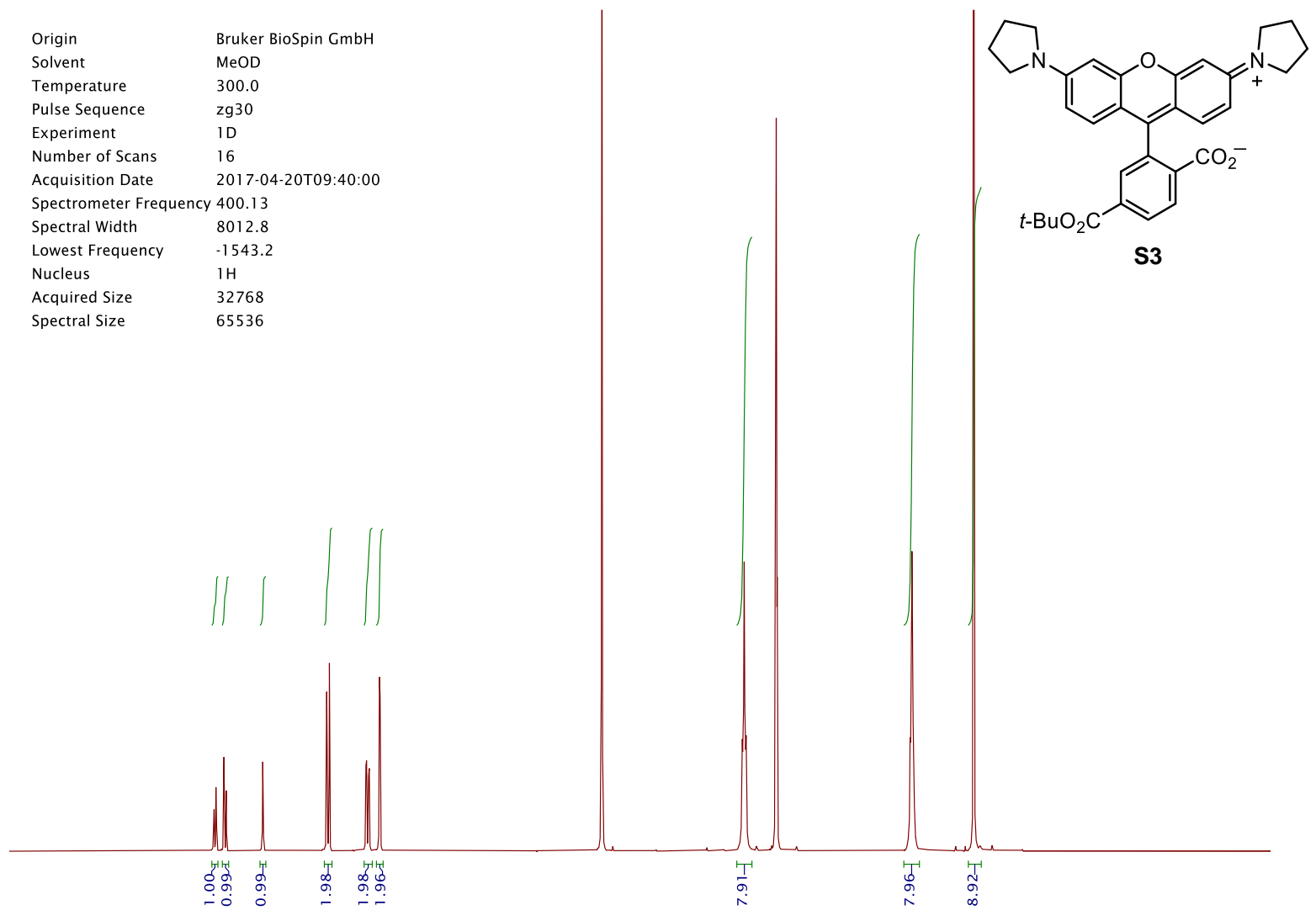

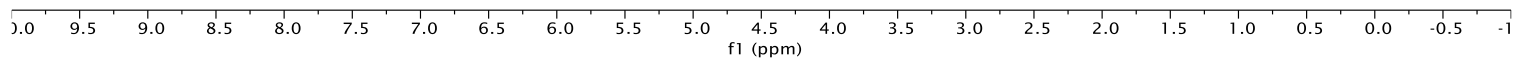

$\begin{array}{ll} & \text { Bruker BioSpin GmbH } \\ \text { Origin } & \text { MeOD } \\ \text { Solvent } & 300.0 \\ \text { Temperature } & \text { zgpg30 } \\ \text { Pulse Sequence } & 1 \mathrm{D} \\ \text { Experiment } & 4096 \\ \text { Number of Scans } & 2019-03-26 \mathrm{~T} 23: 55: 00 \\ \text { Acquisition Date } & 24038.5 \\ \text { Spectrometer Frequency } 100.62 \\ \text { Spectral Width } & -1817.9 \\ \text { Lowest Frequency } & 13 \mathrm{C} \\ \text { Nucleus } & 32768 \\ \text { Acquired Size } & 65536\end{array}$

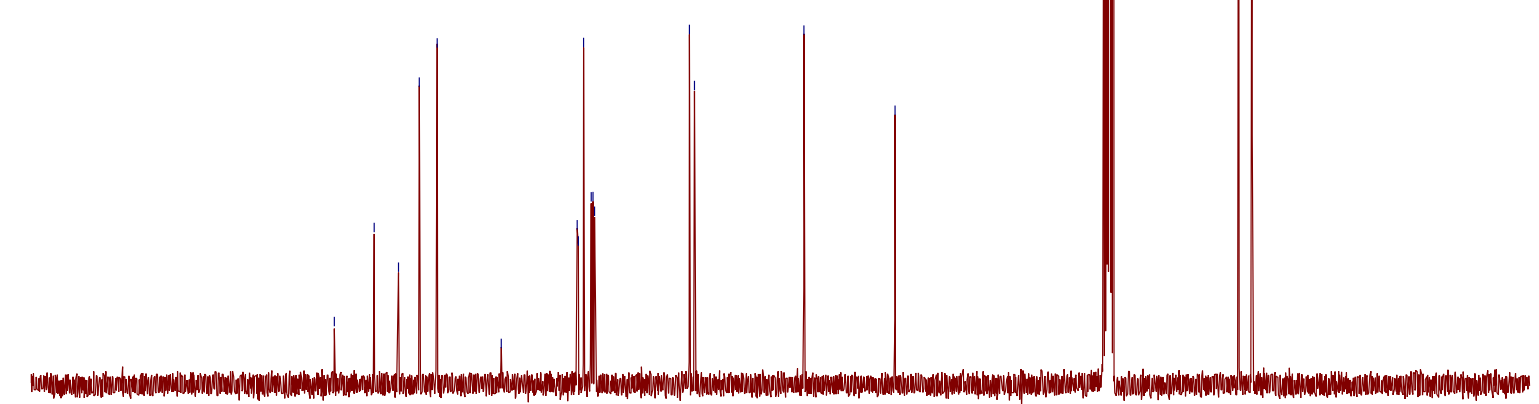

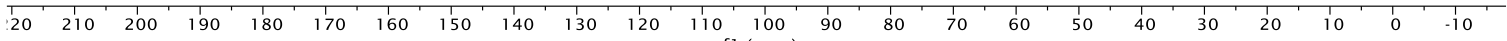




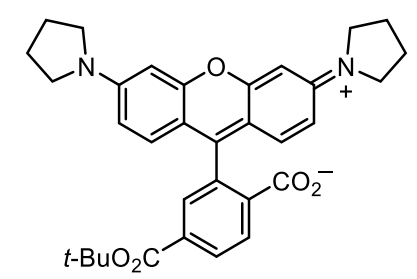

S3

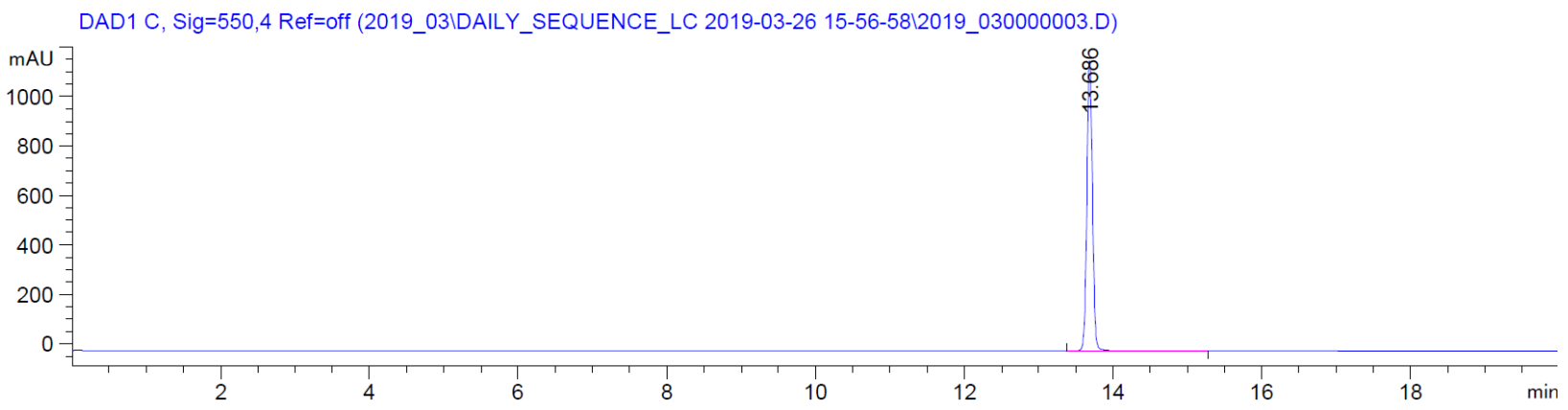

*MSD2 SPC, time=13.681:13.772 of C:ICHEM3211IDATAI2019_03IDAILY_SEQUENCE_LC 2019-03-26 15-56-5812019_0300000003.D ES-

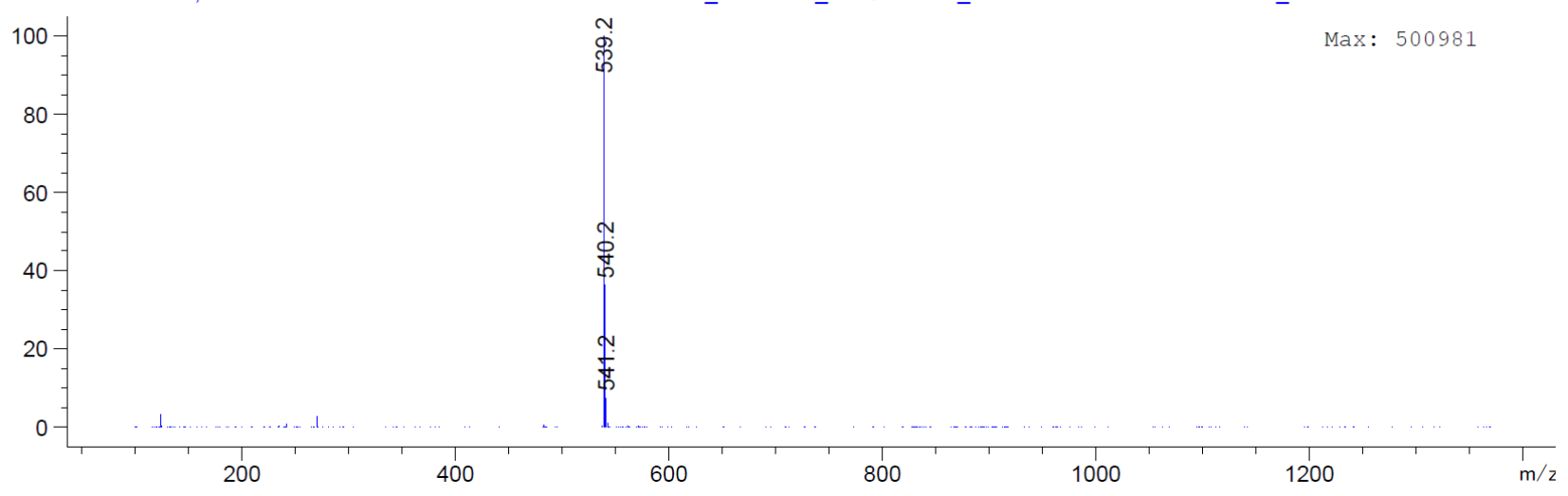



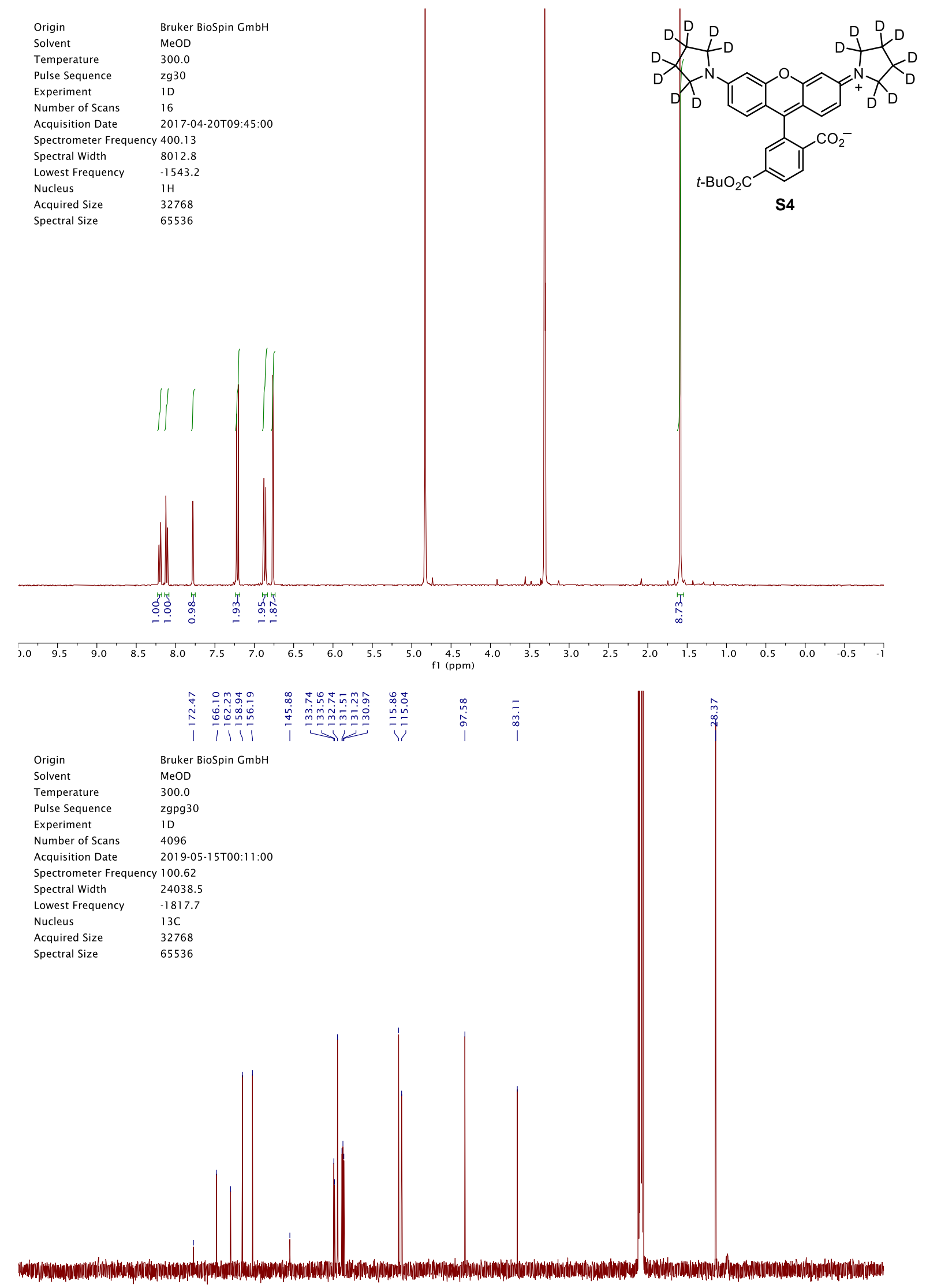

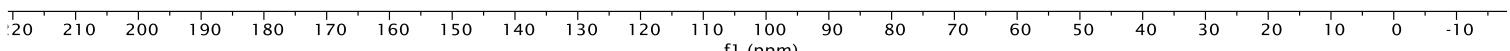




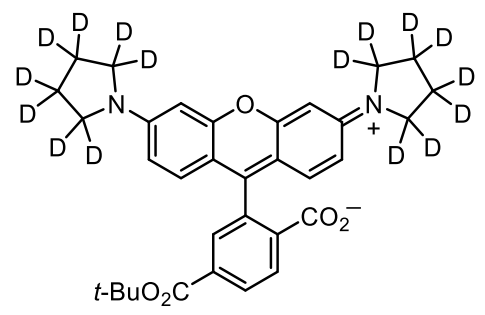

S4

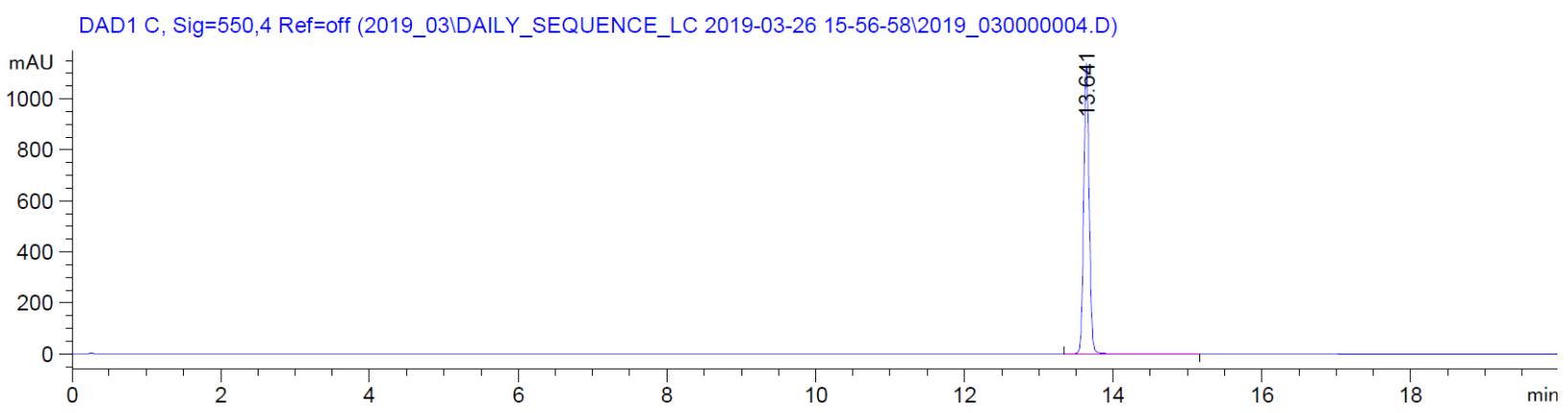

*MSD2 SPC, time=13.641:13.732 of C:ICHEM32I1IDATAI2019_03IDAILY_SEQUENCE_LC 2019-03-26 15-56-5812019_0300000004.D ES-

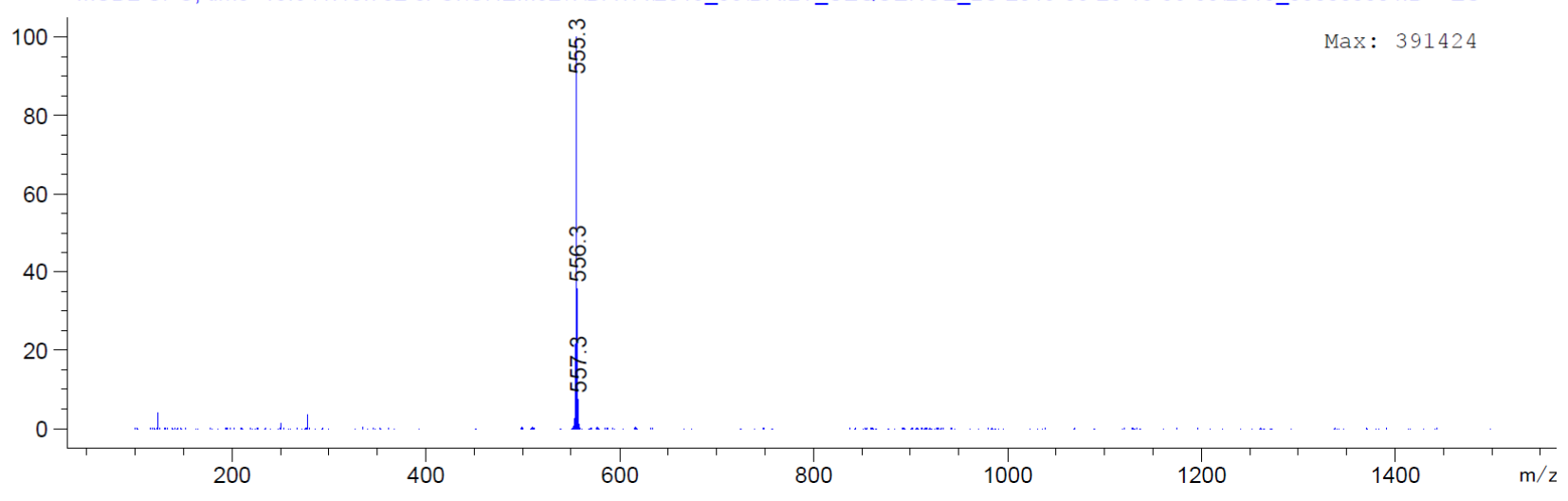




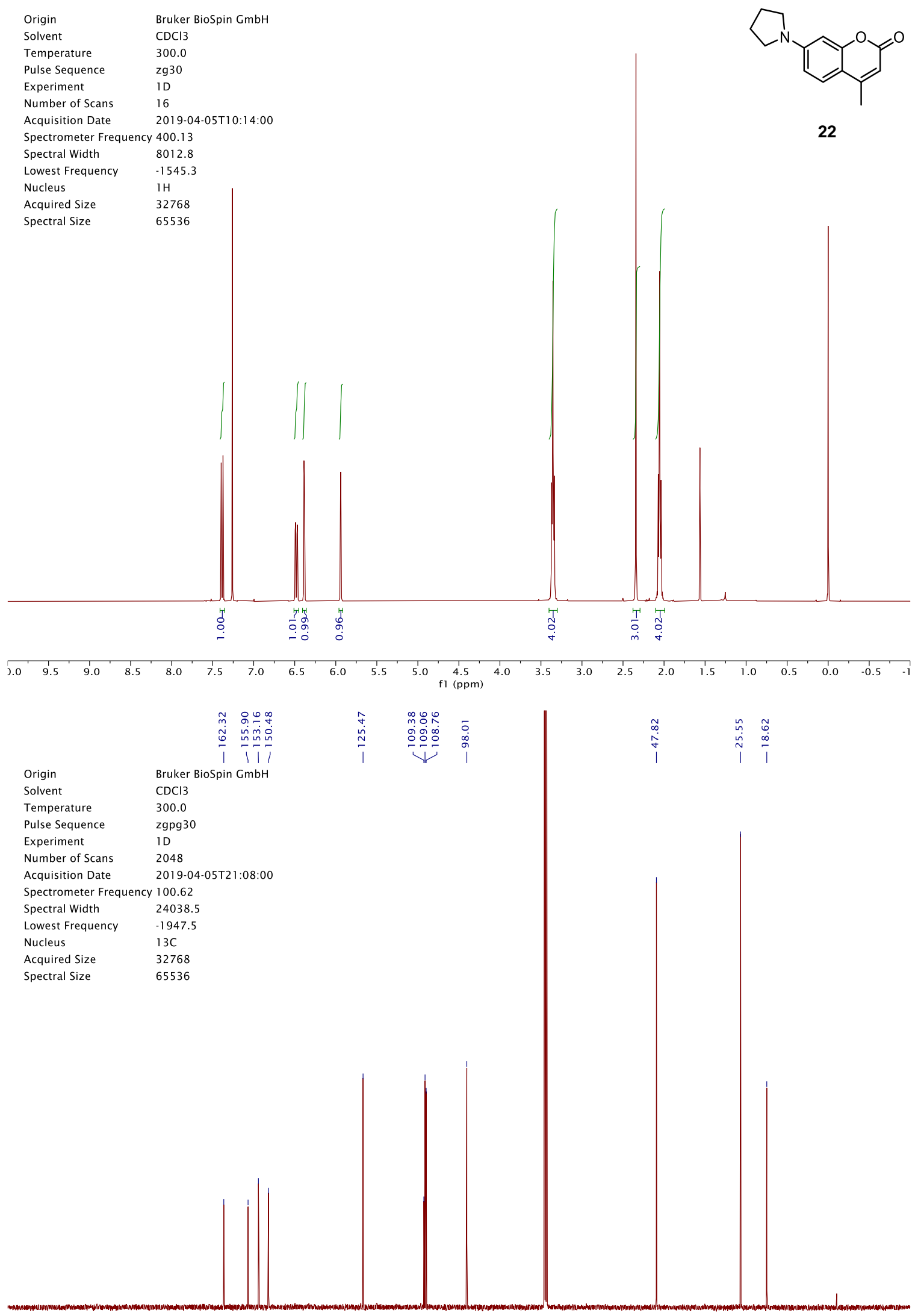

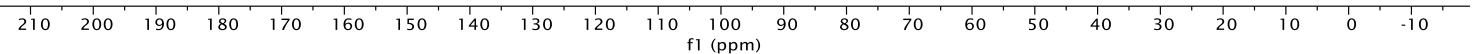




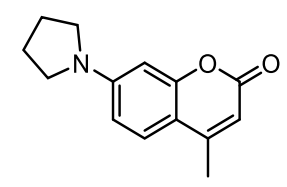

22

DAD1 C, Sig=375,4 Ref=off (2019_04IDAILY_SEQUENCE_LC 2019-04-02 09-15-1312019_040000002.D)

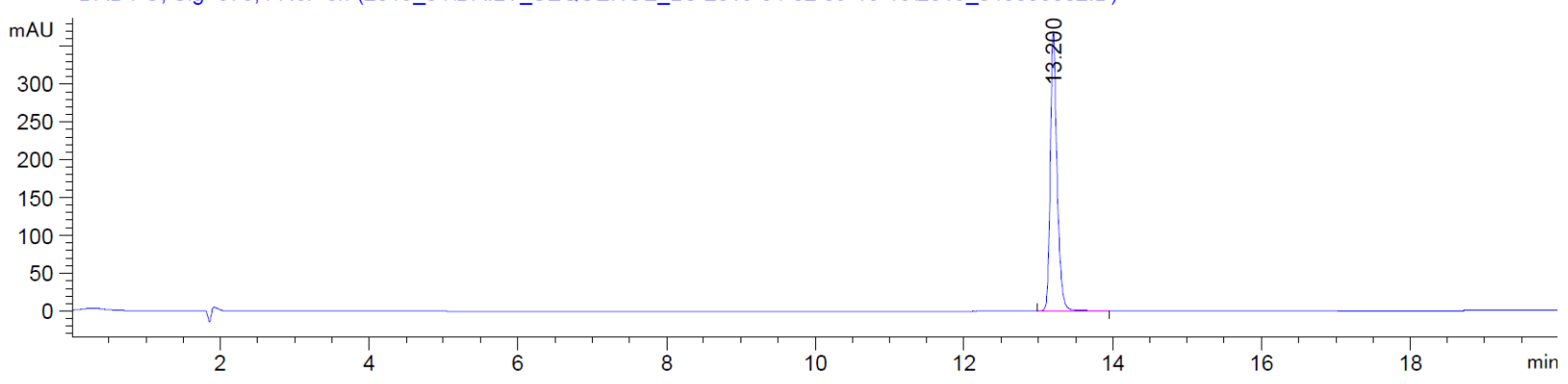

*MSD2 SPC, time=13.205:13.296 of C:ICHEM32I1IDATAI2019_04IDAILY_SEQUENCE_LC 2019-04-02 09-15-13I2019_040000002.D ES-

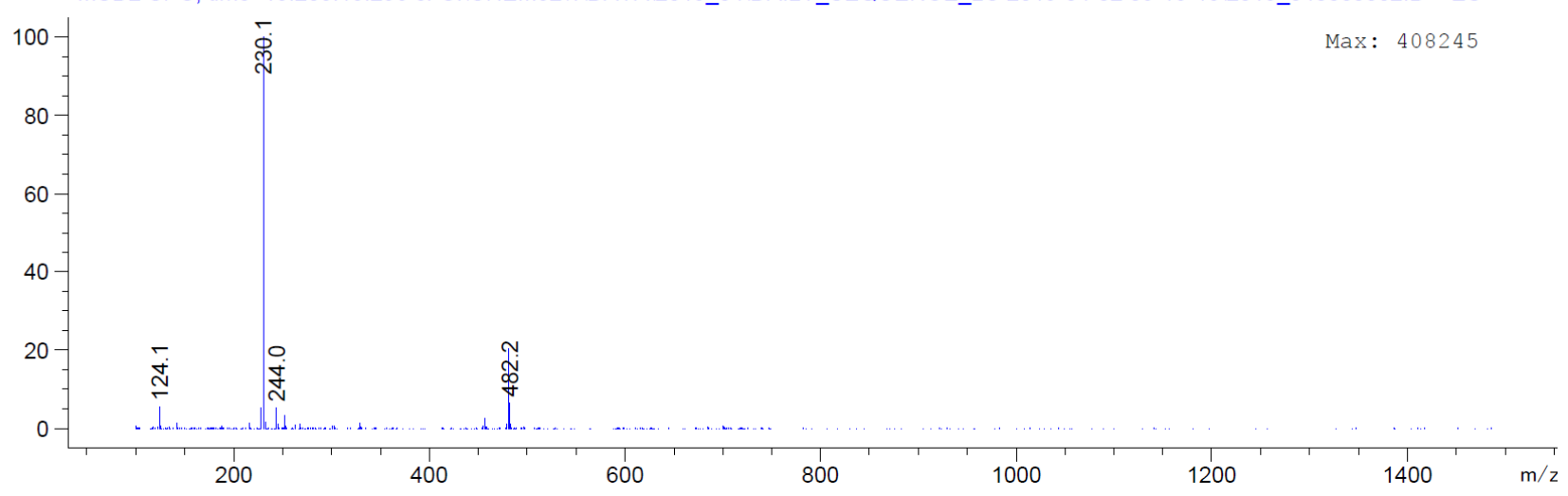




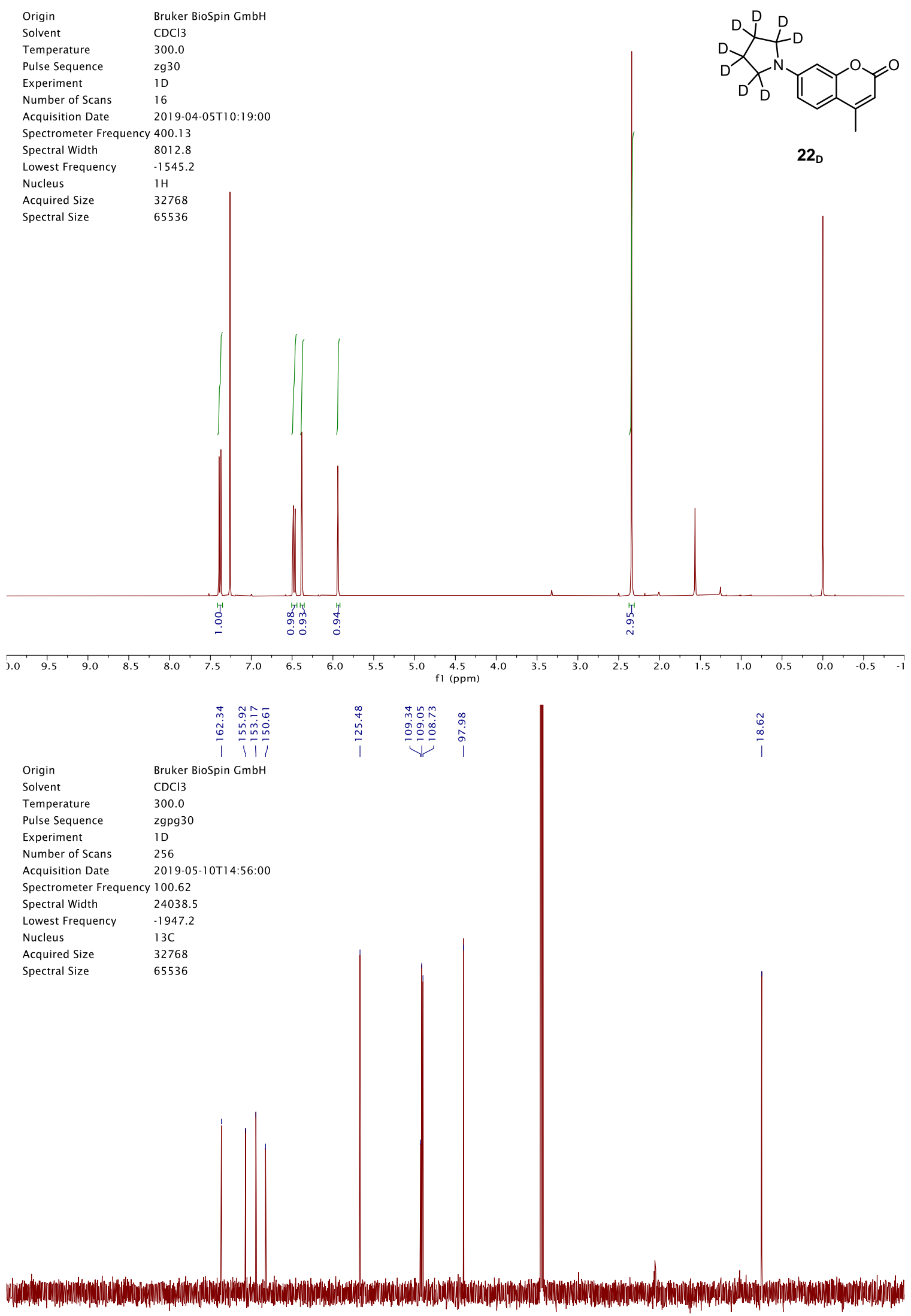

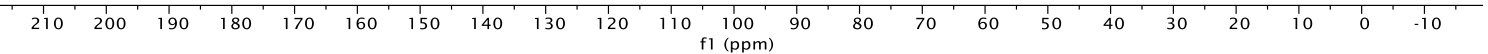




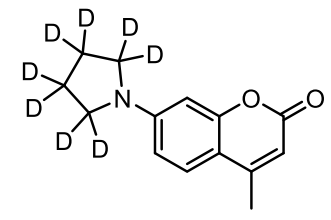

22D

DAD1 C, Sig=375,4 Ref=off (2019_04IDAILY_SEQUENCE_LC 2019-04-02 14-51-4012019_040000004.D)

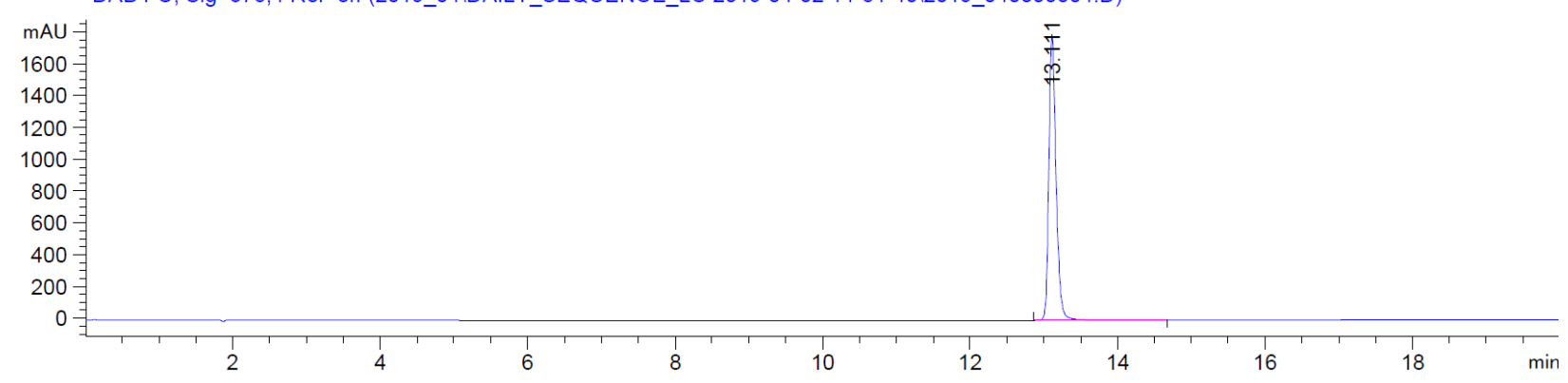

*MSD2 SPC, time=13.100:13.209 of C:ICHEM32I1IDATAI2019_04IDAILY_SEQUENCE_LC 2019-04-02 14-51-4012019_0400000004.D ES-

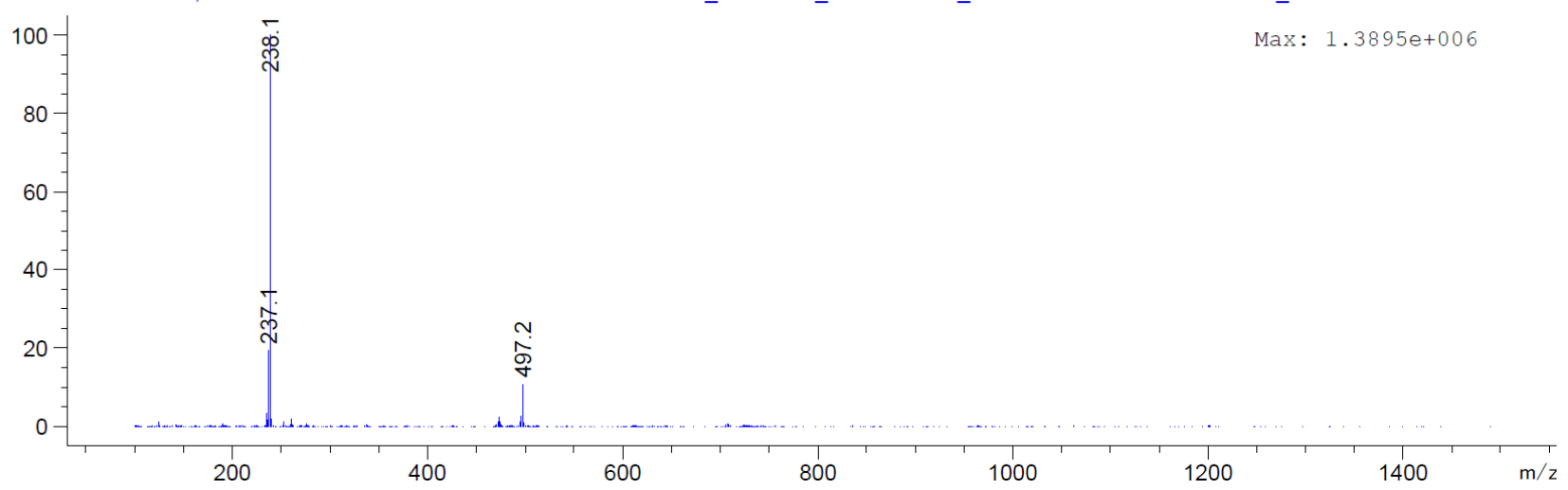




$\begin{array}{ll}\text { Origin } & \text { Bruker BioSpin GmbH } \\ \text { Solvent } & \text { MeOD } \\ \text { Temperature } & 300.0 \\ \text { Pulse Sequence } & \mathrm{zg} 30 \\ \text { Experiment } & 1 \mathrm{D} \\ \text { Number of Scans } & 16 \\ \text { Acquisition Date } & 2019-04-08 \mathrm{~T} 11: 32: 00 \\ \text { Spectrometer Frequency } 400.13 \\ \text { Spectral Width } & 8012.8 \\ \text { Lowest Frequency } & -1543.2 \\ \text { Nucleus } & 1 \mathrm{H} \\ \text { Acquired Size } & 32768 \\ \text { Spectral Size } & 65536\end{array}$

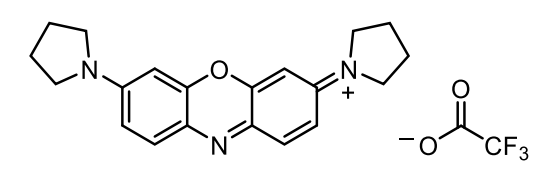

23
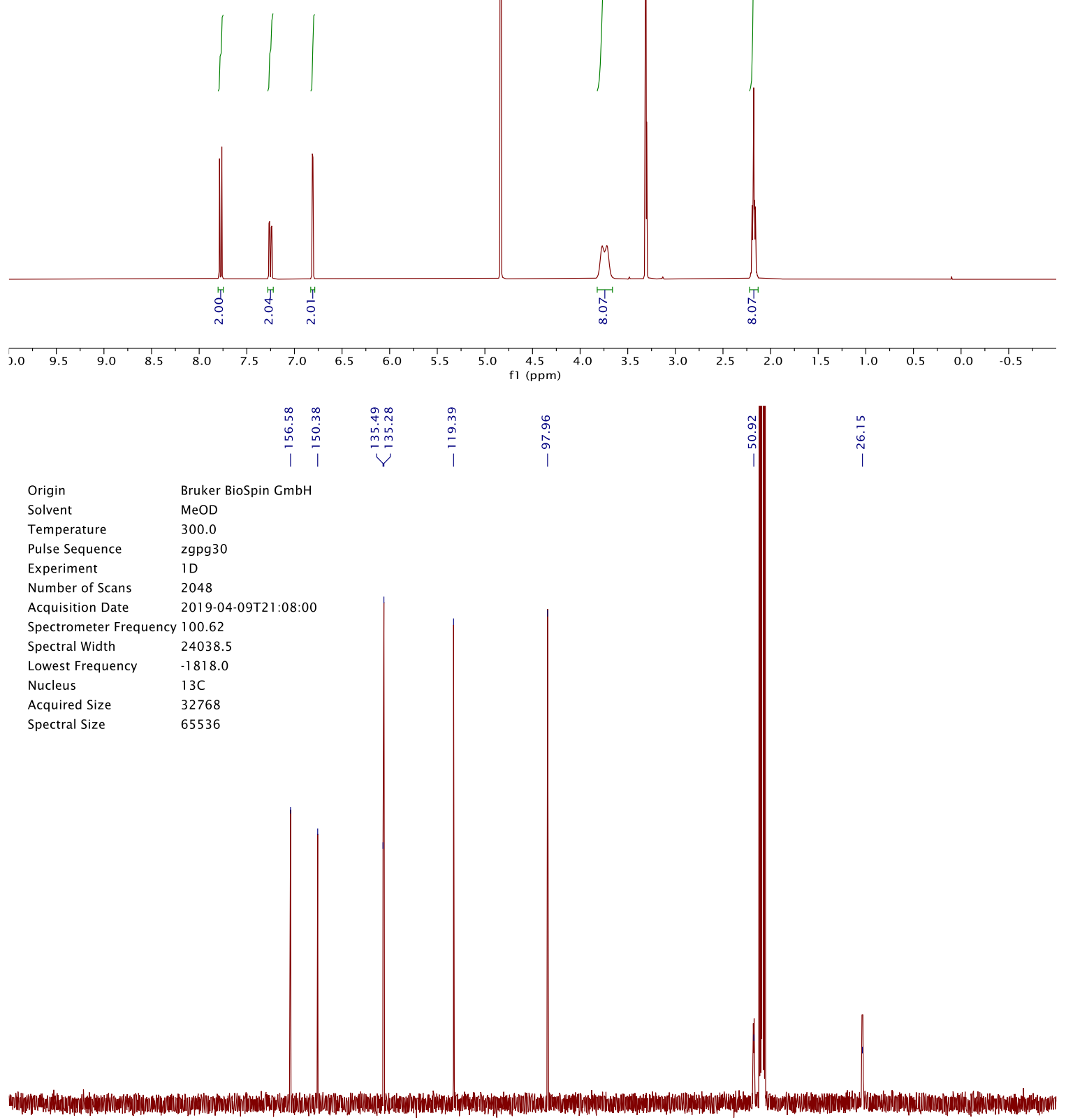

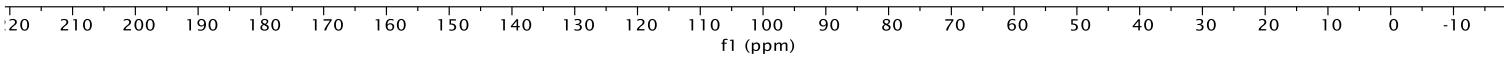




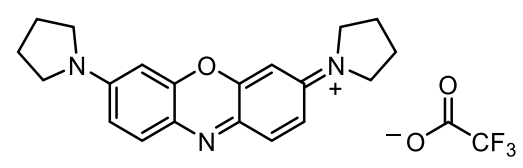

23

DAD1 E, Sig=650,4 Ref=off (2019_04IDAILY_SEQUENCE_LC 2019-04-04 10-58-07/2019_040000002.D)

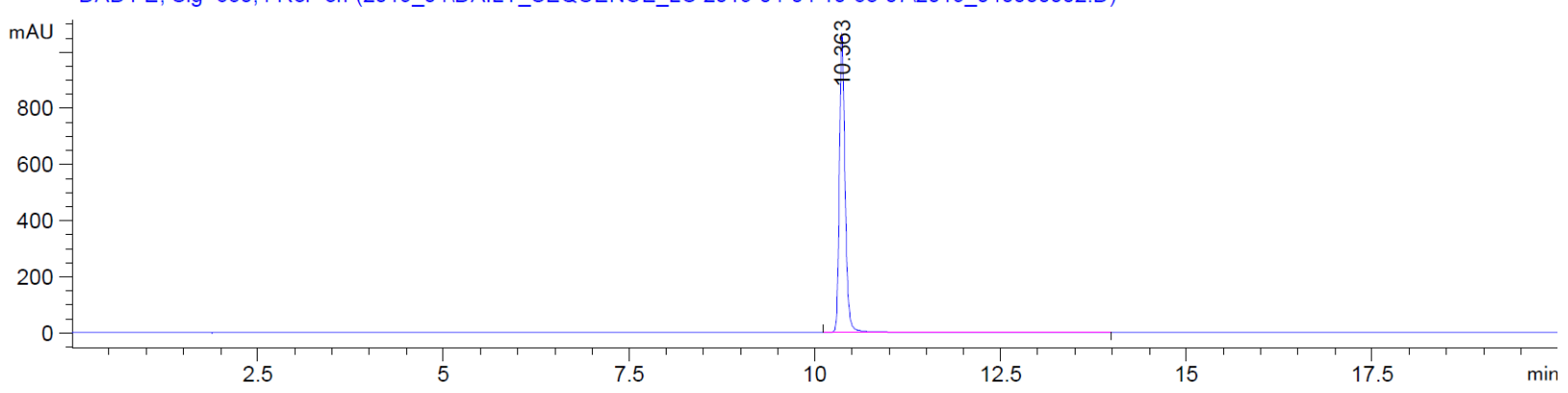

${ }^{*}$ MSD2 SPC, time=10.353:10.462 of C:ICHEM32I1IDATAI2019_04IDAILY_SEQUENCE_LC 2019-04-04 10-58-0712019_0400000002.D ES-

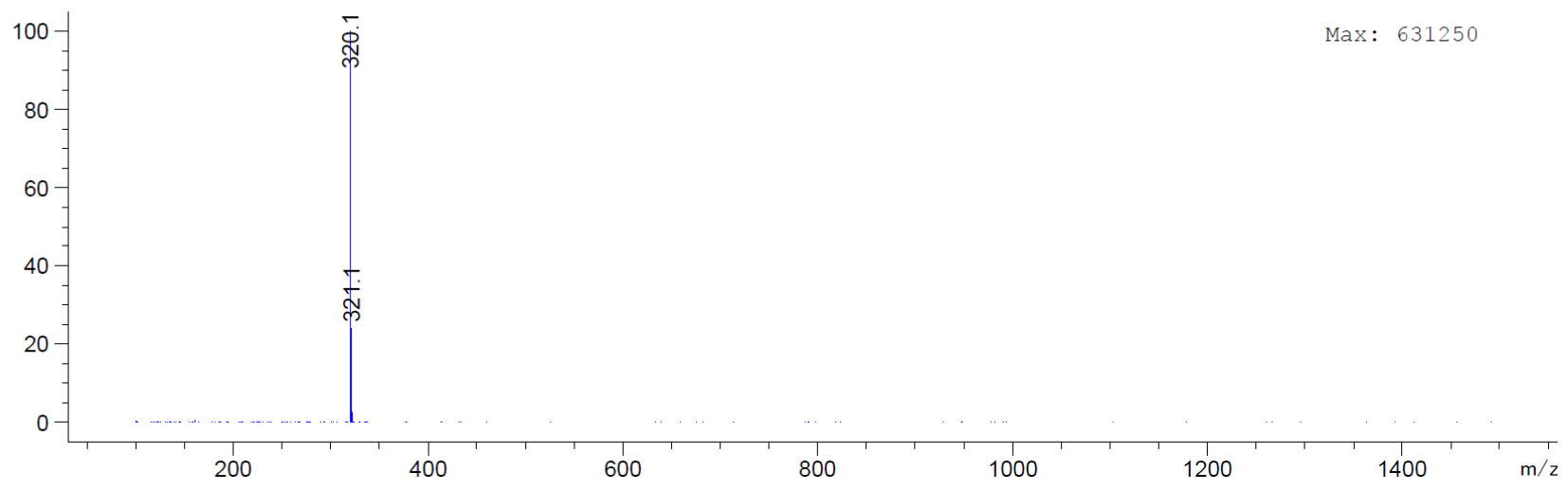



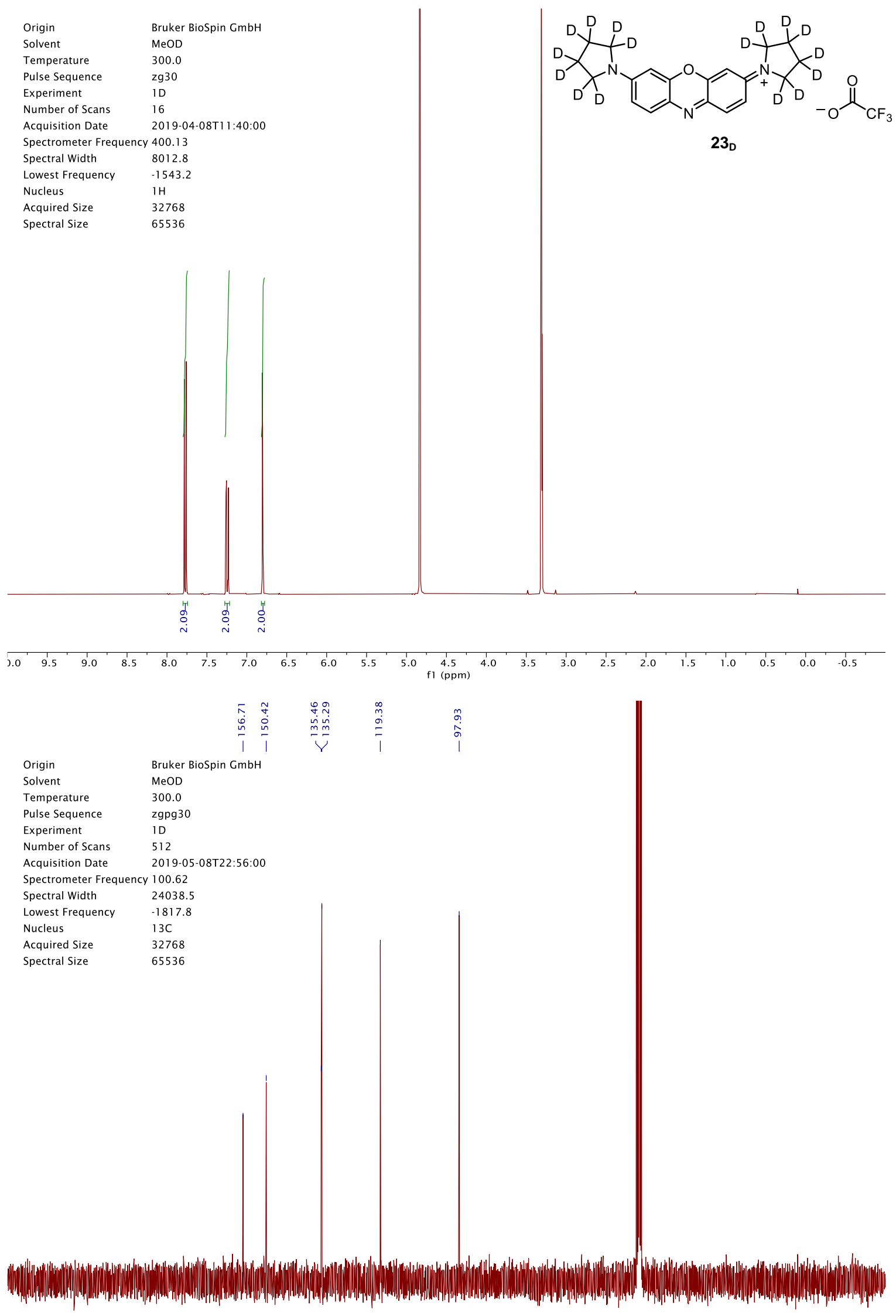

$\begin{array}{llllllllllllllllllllllllllllllll}1 & 20 & 210 & 200 & 190 & 180 & 170 & 160 & 150 & 140 & 130 & 120 & 110 & 100 & 90 & 80 & 70 & 60 & 50 & 40 & 30 & 20 & 10 & 0 & -10\end{array}$ 


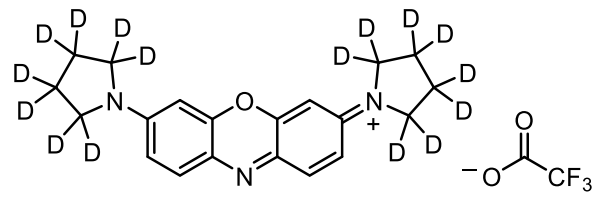

23 D

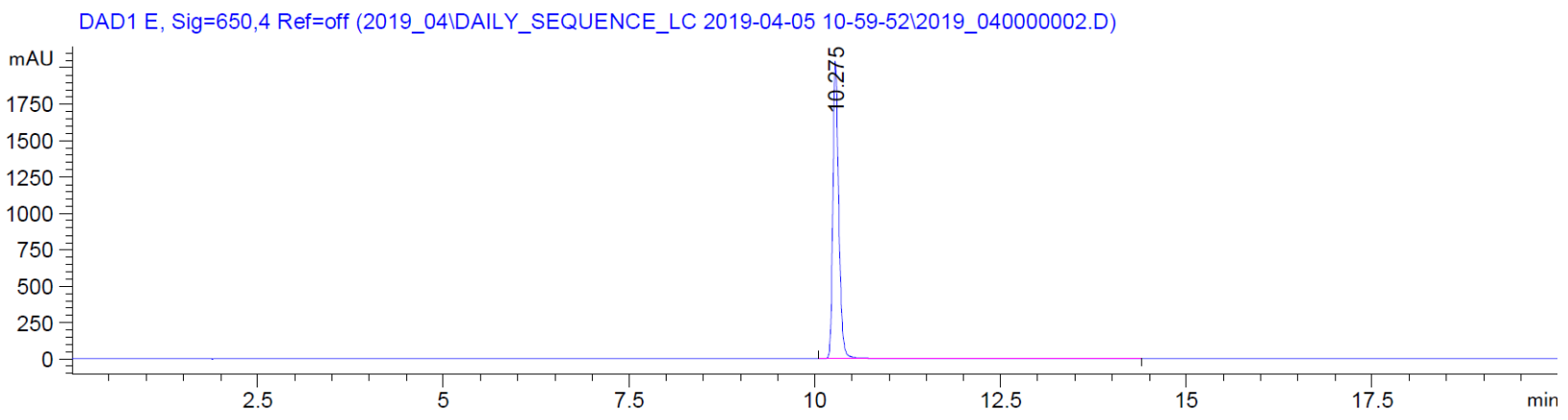

${ }^{*}$ MSD2 SPC, time=10.280:10.371 of C:ICHEM32111DATAI2019_04IDAILY_SEQUENCE_LC 2019-04-05 10-59-5212019_0400000002.D ES-

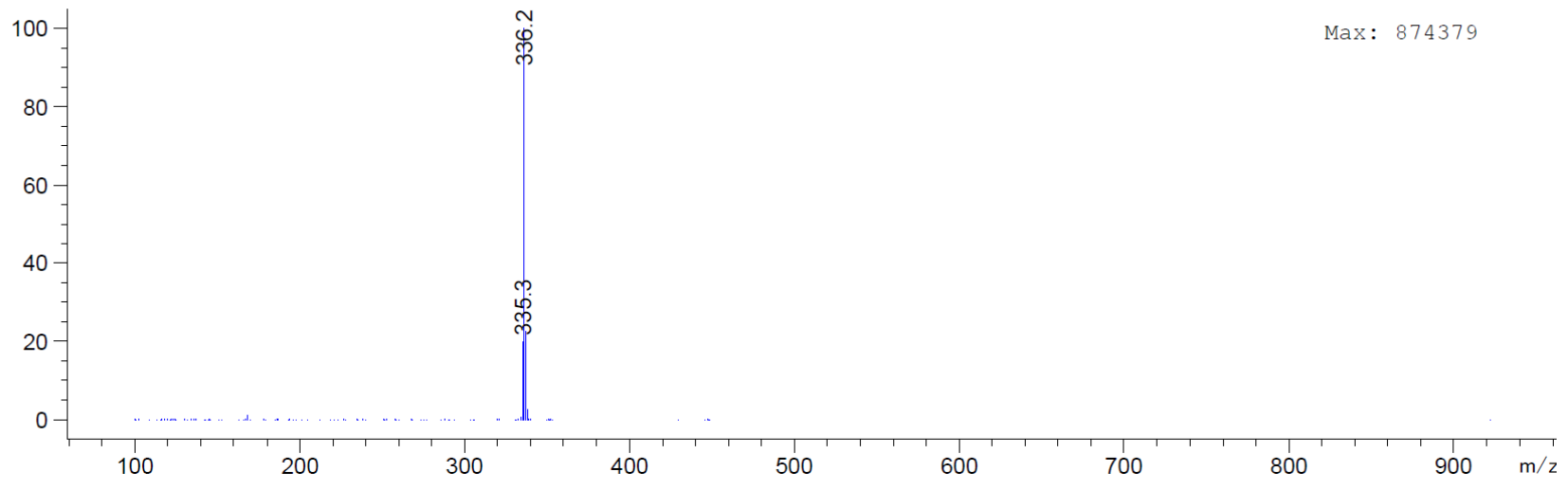



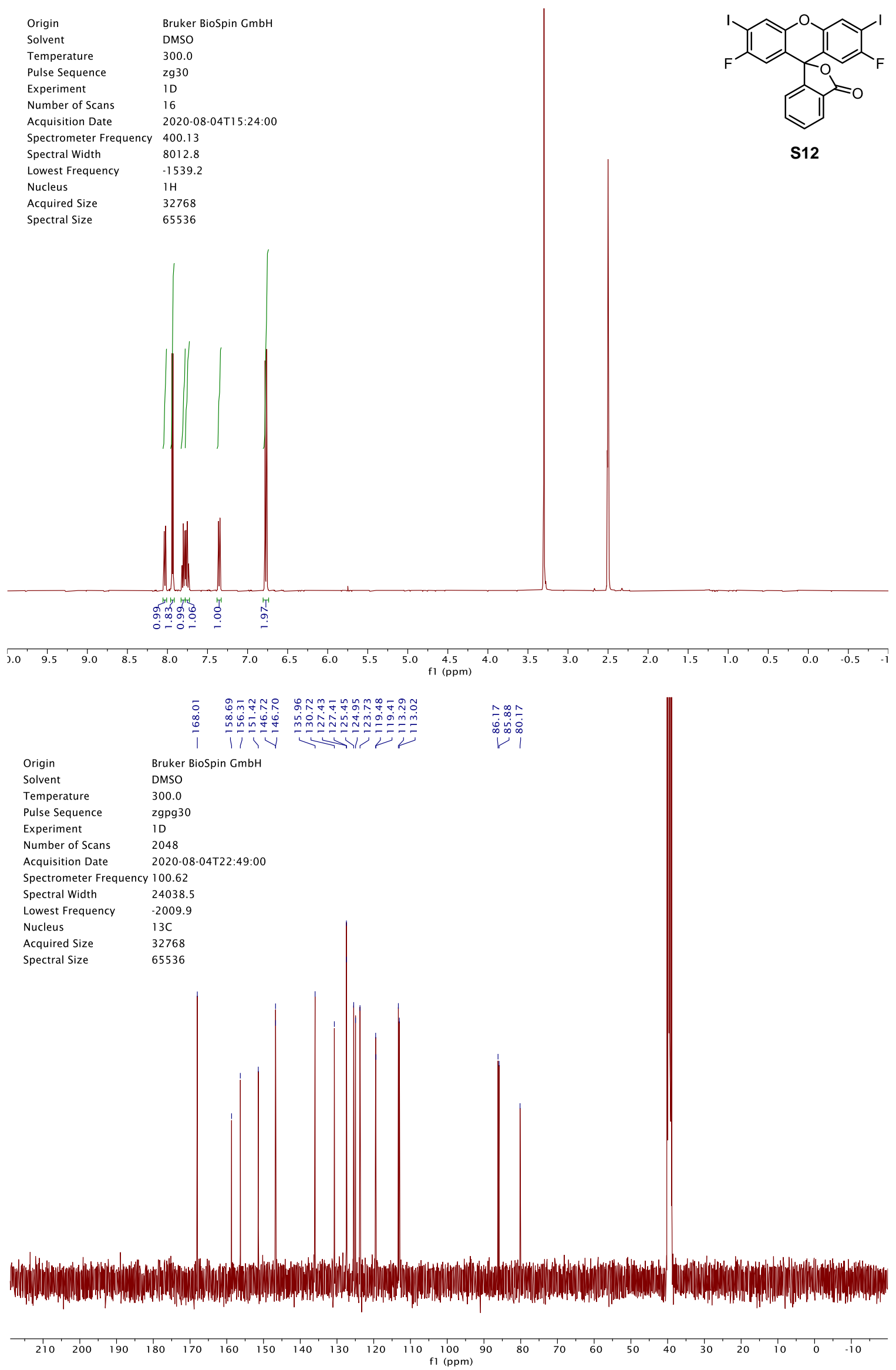

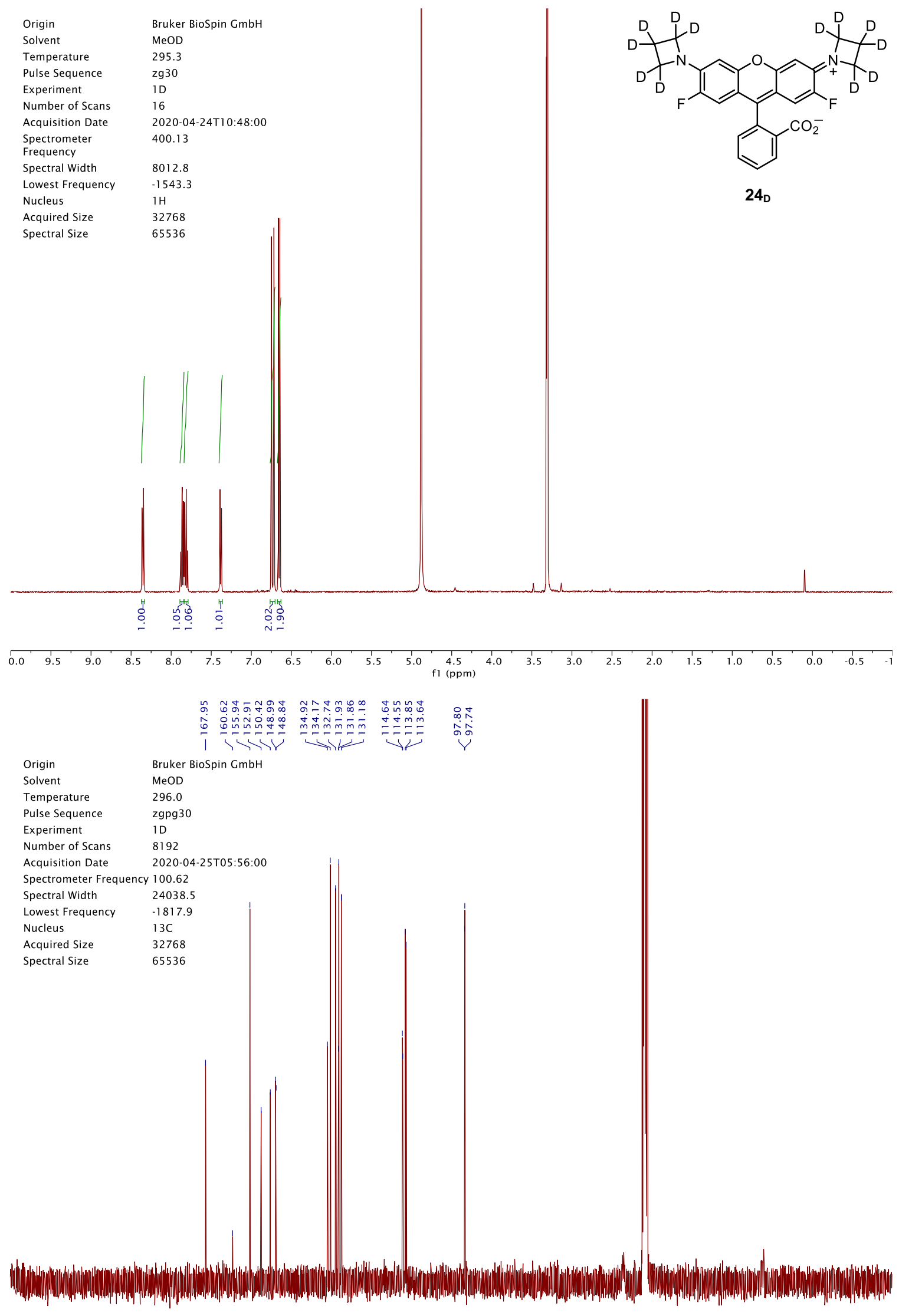

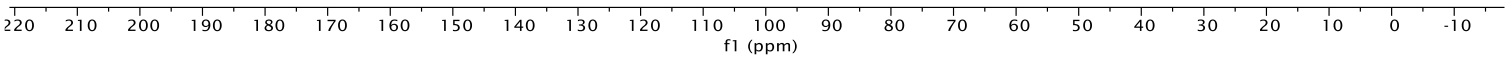




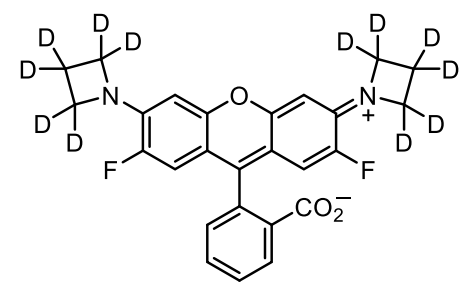

24D

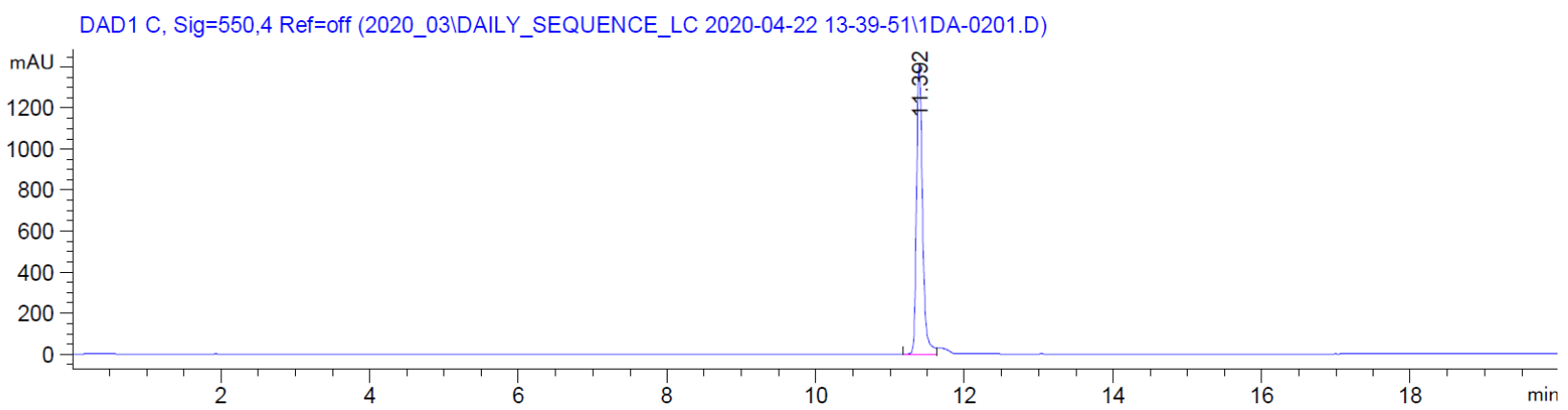

*MSD2 SPC, time=11.498:11.607 of C:ICHEM32I1IDATAL2020_03IDAILY_SEQUENCE_LC 2020-04-22 13-39-5111DA-0201.D ES-API, P

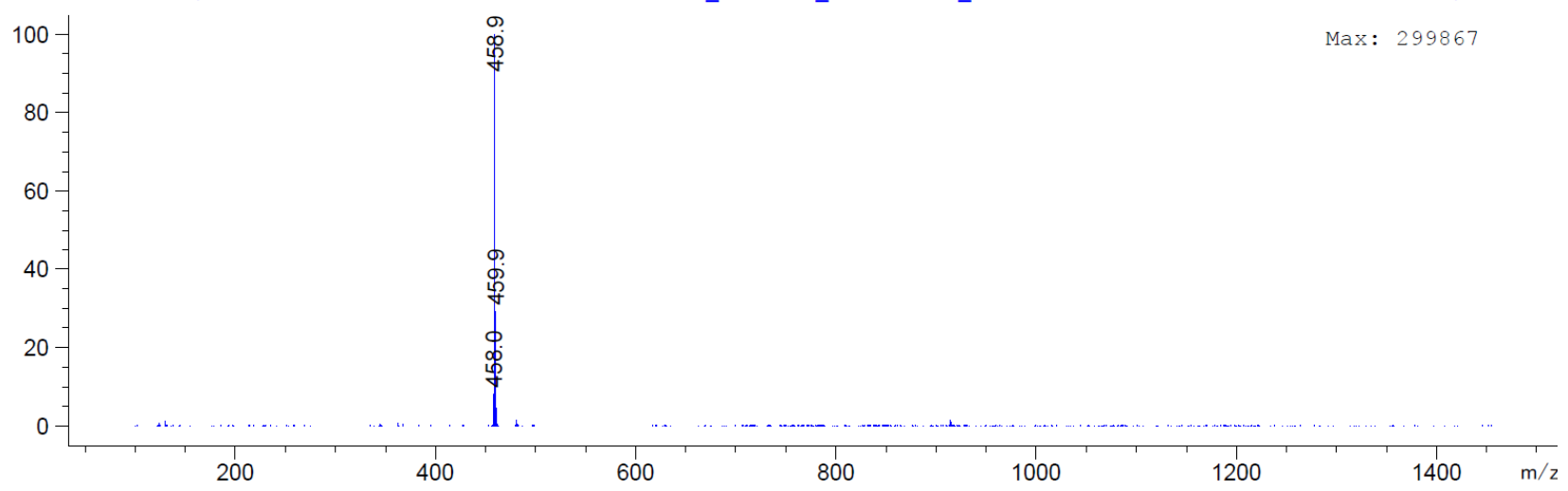



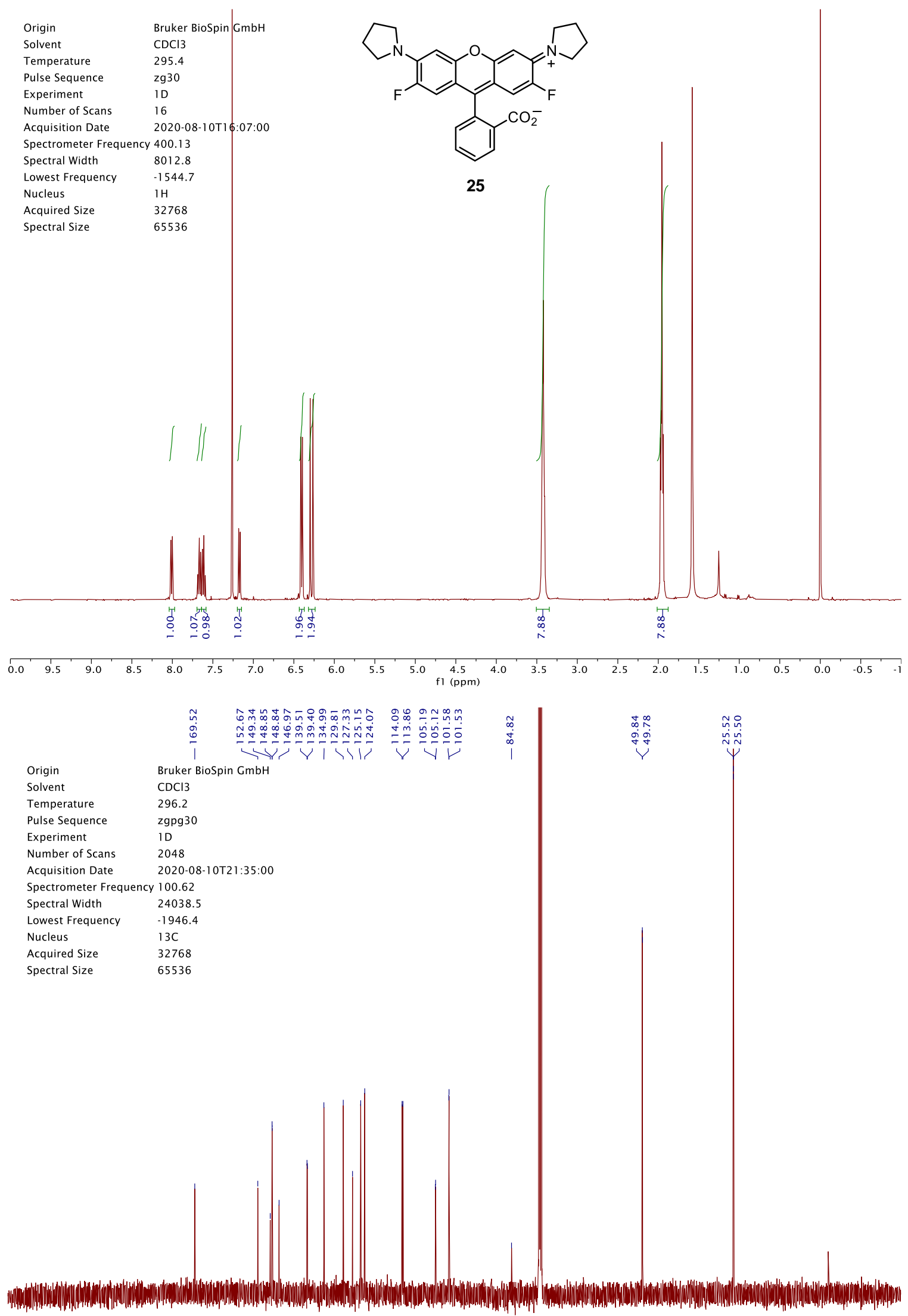

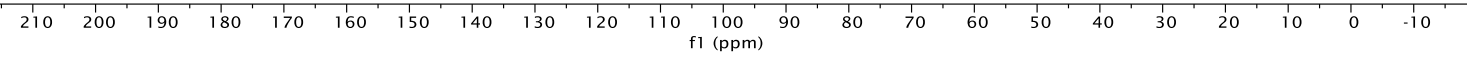




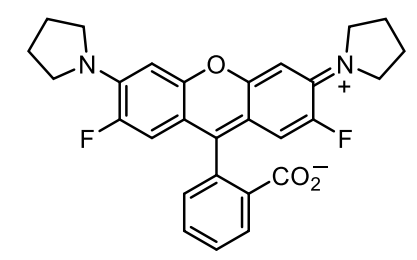

25

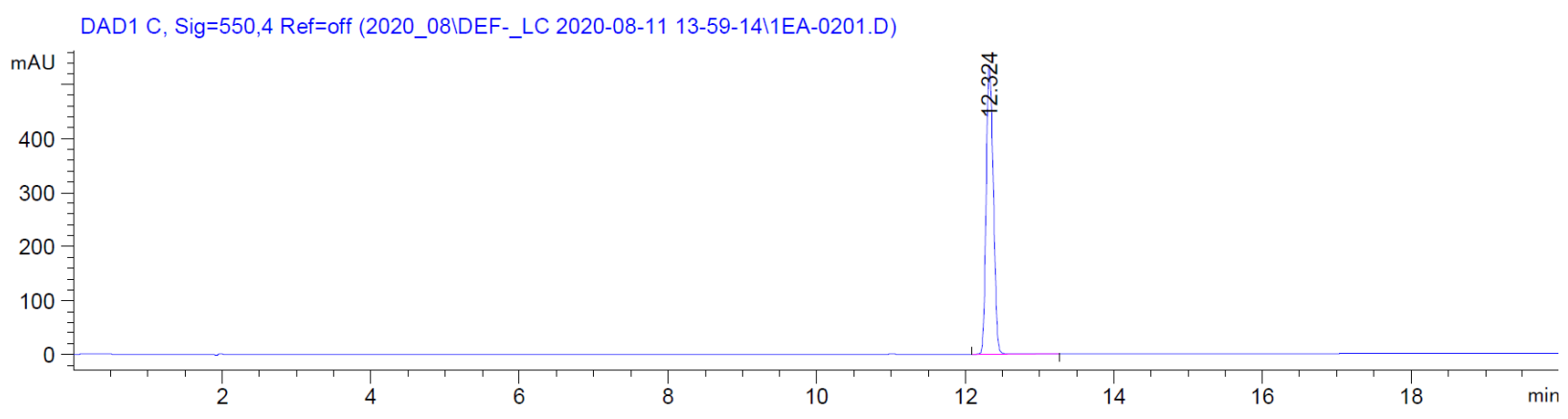

*MSD2 SPC, time=12.388:12.606 of C:ICHEM32I1IDATAI2020_08IDEF-_LC 2020-08-11 13-59-14l1EA-0201.D ES-API, Pos, Scan,

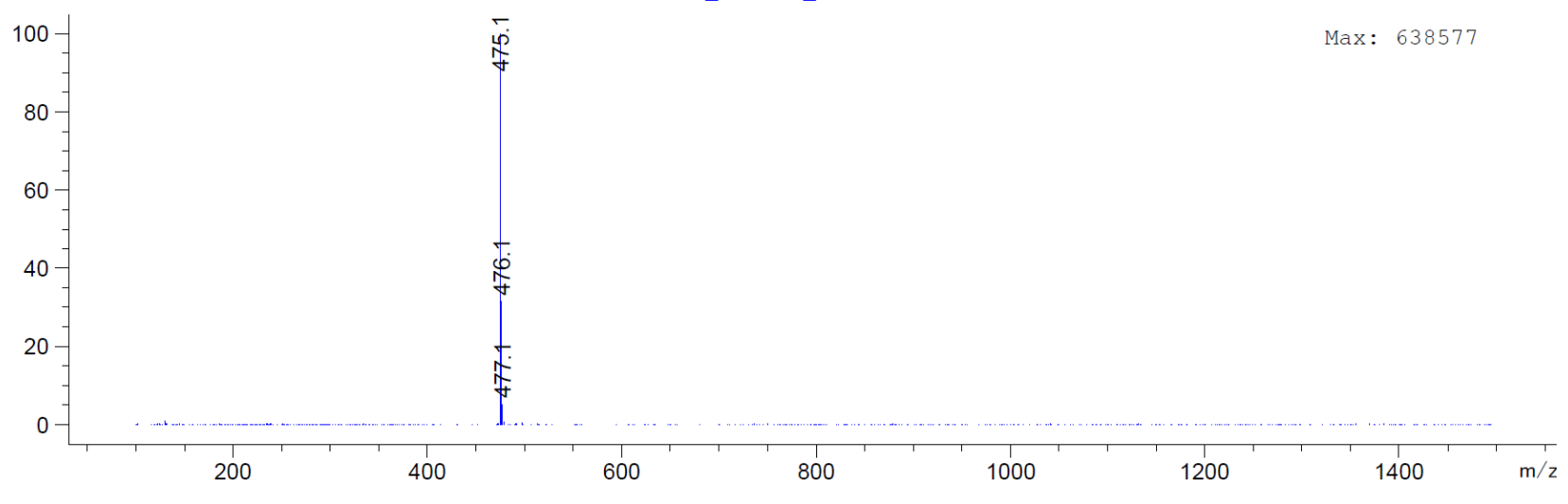



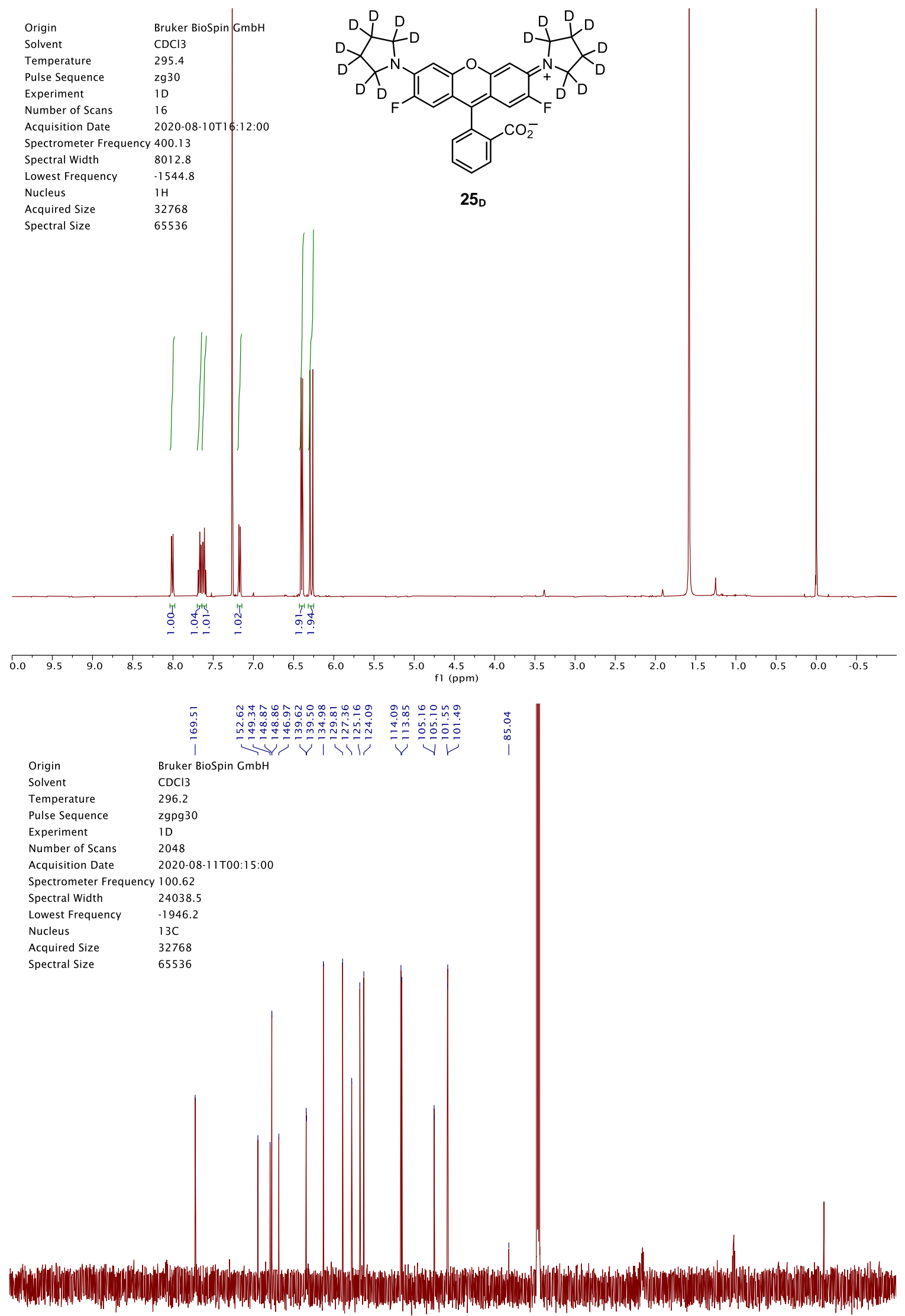

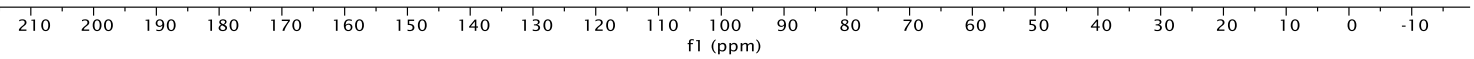




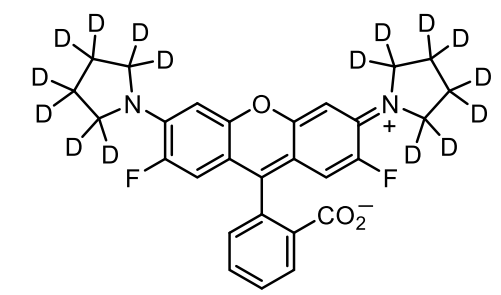

25D

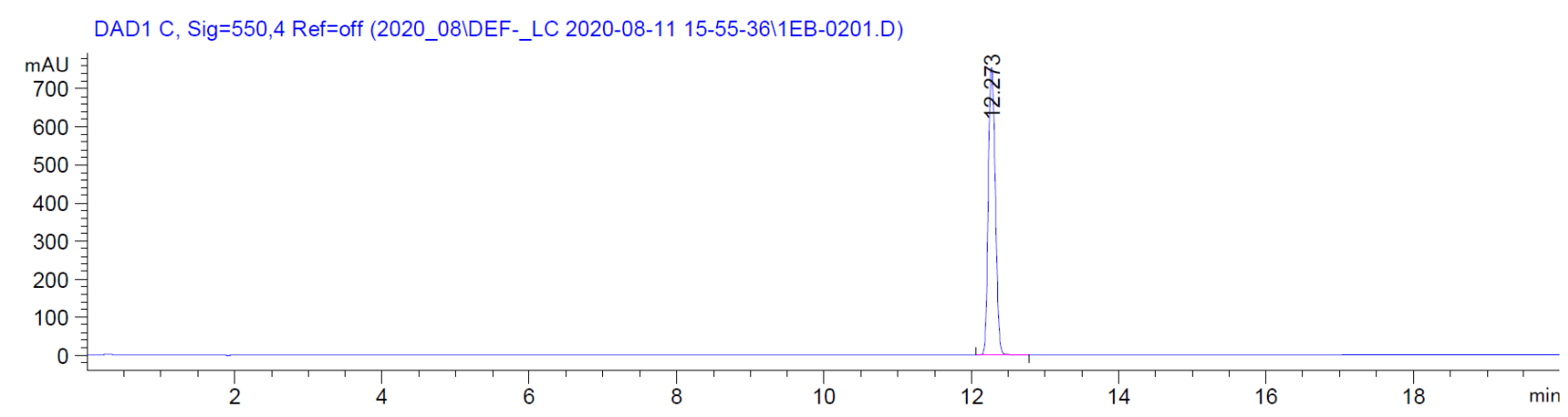

*MSD2 SPC, time=12.334:12.552 of C:ICHEM32I1IDATAI2020_08IDEF-_LC 2020-08-11 15-55-36|1EB-0201.D ES-API, Pos, Scan,

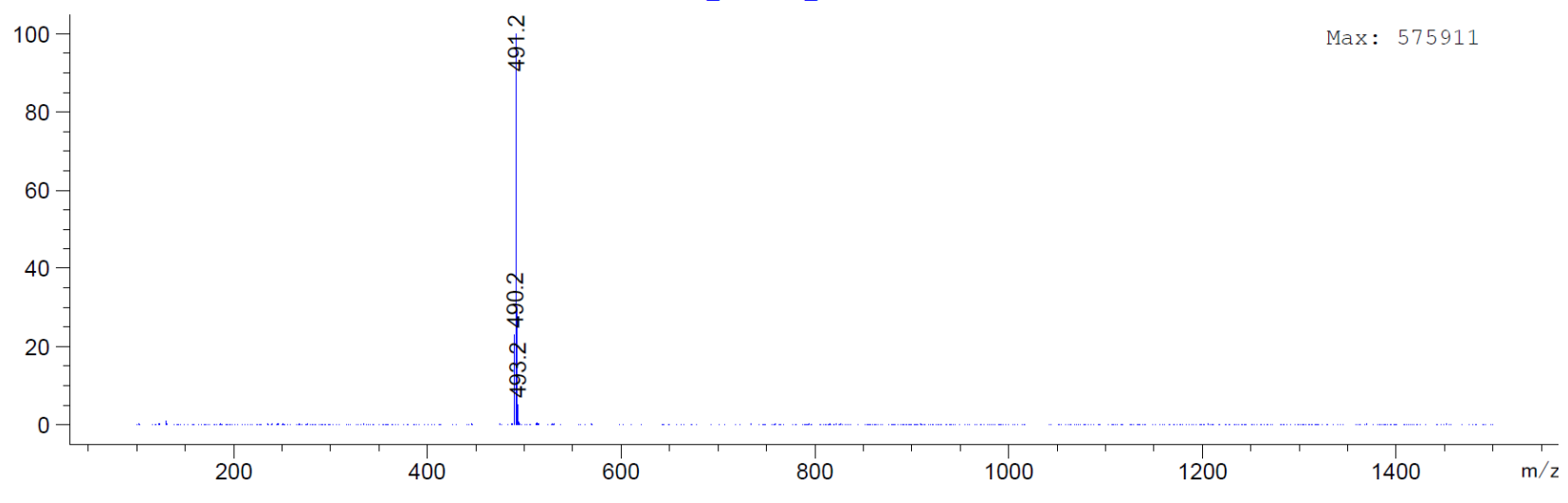



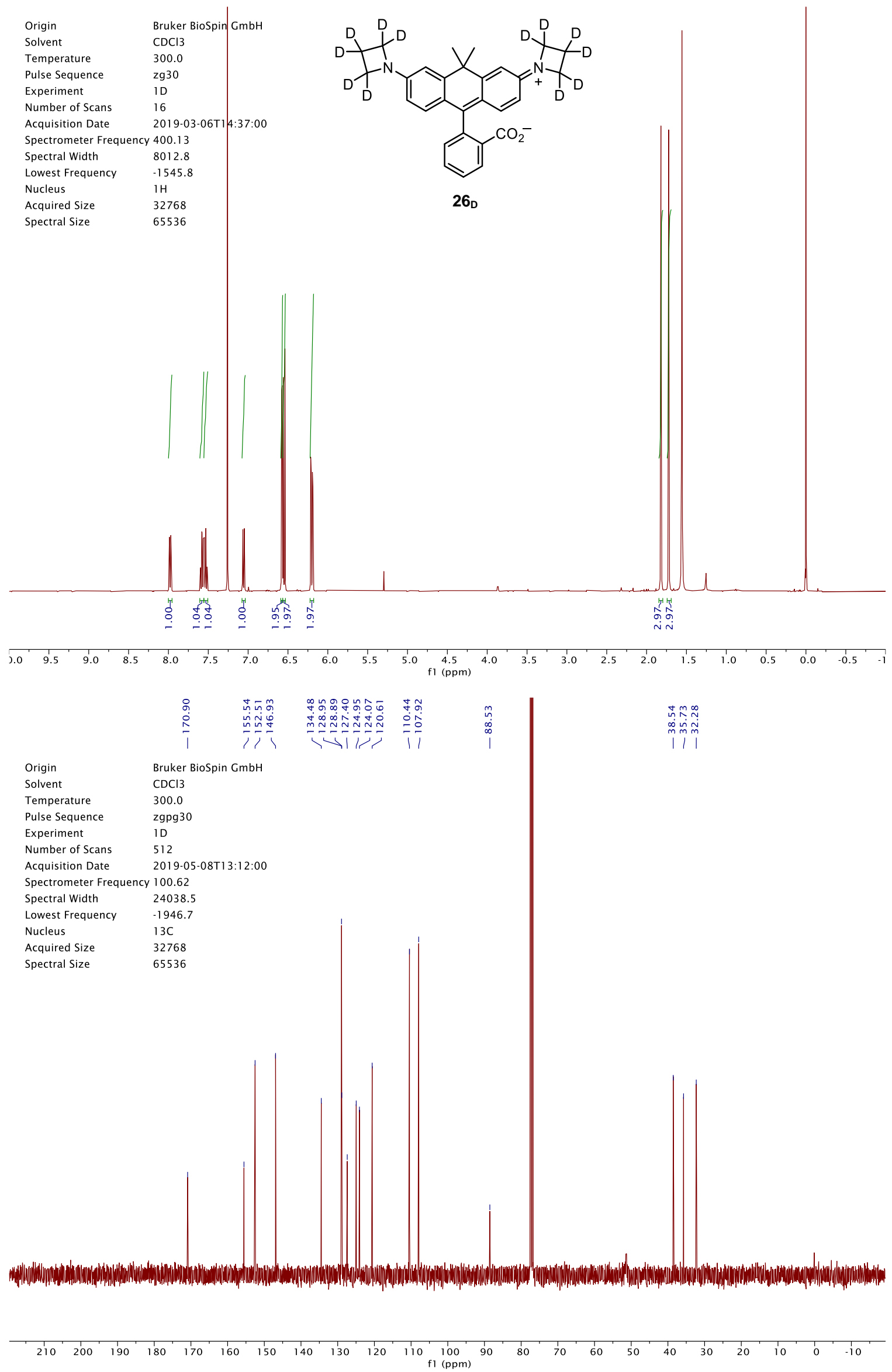


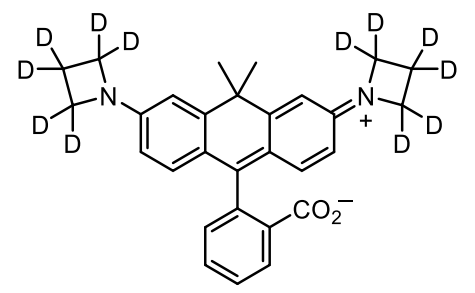

26 D

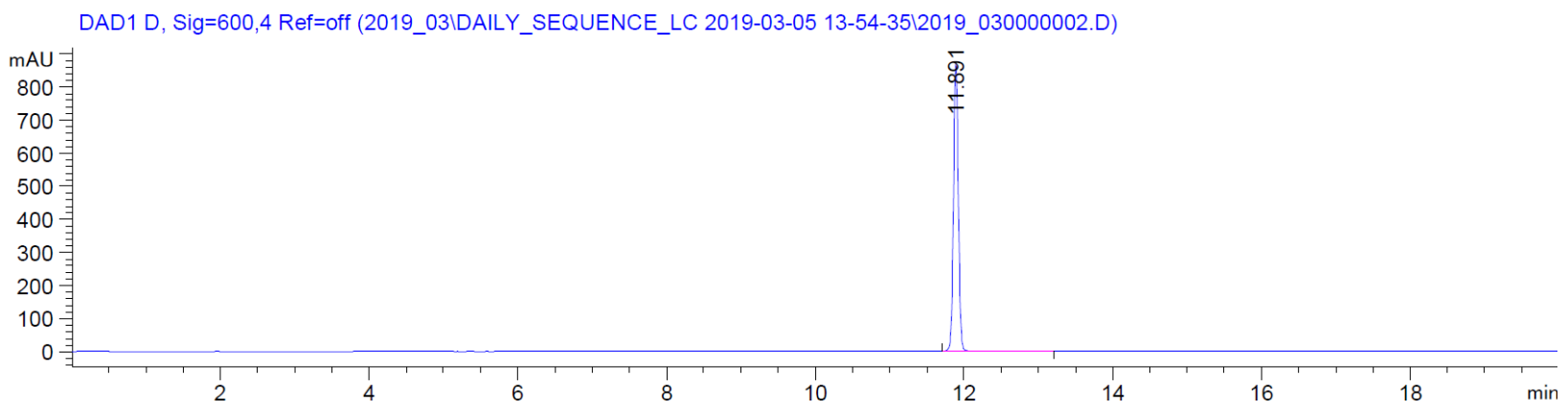

*MSD2 SPC, time=11.879:12.006 of C:ICHEM32I1IDATAI2019_03IDAILY_SEQUENCE_LC 2019-03-05 13-54-3512019_030000002.D ES-

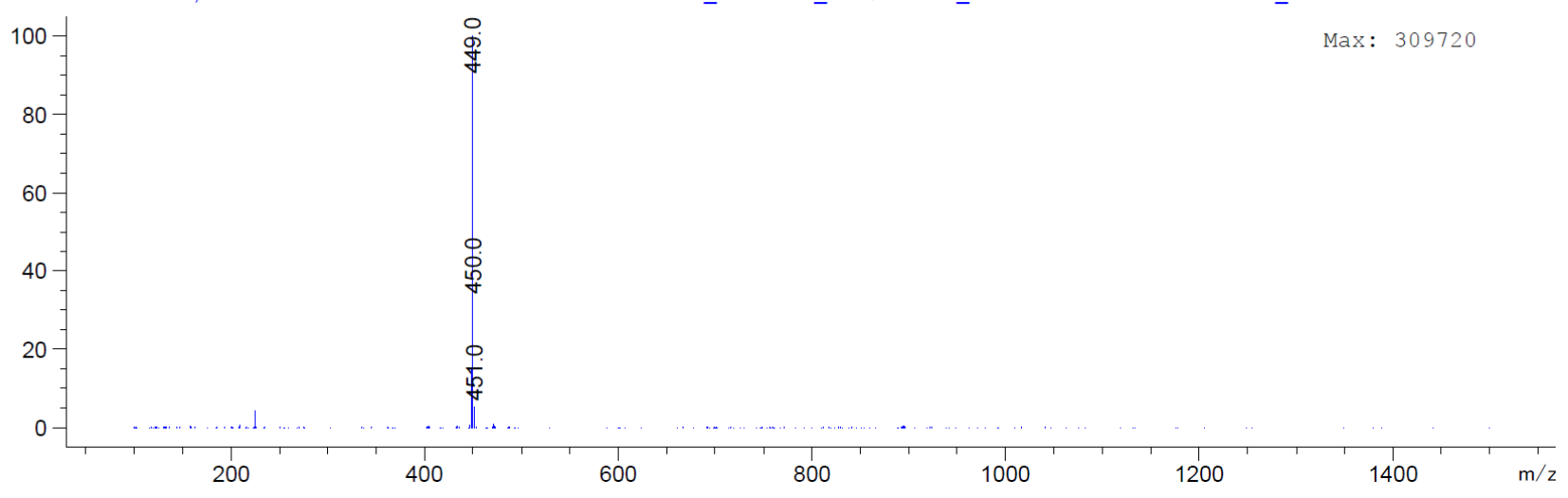



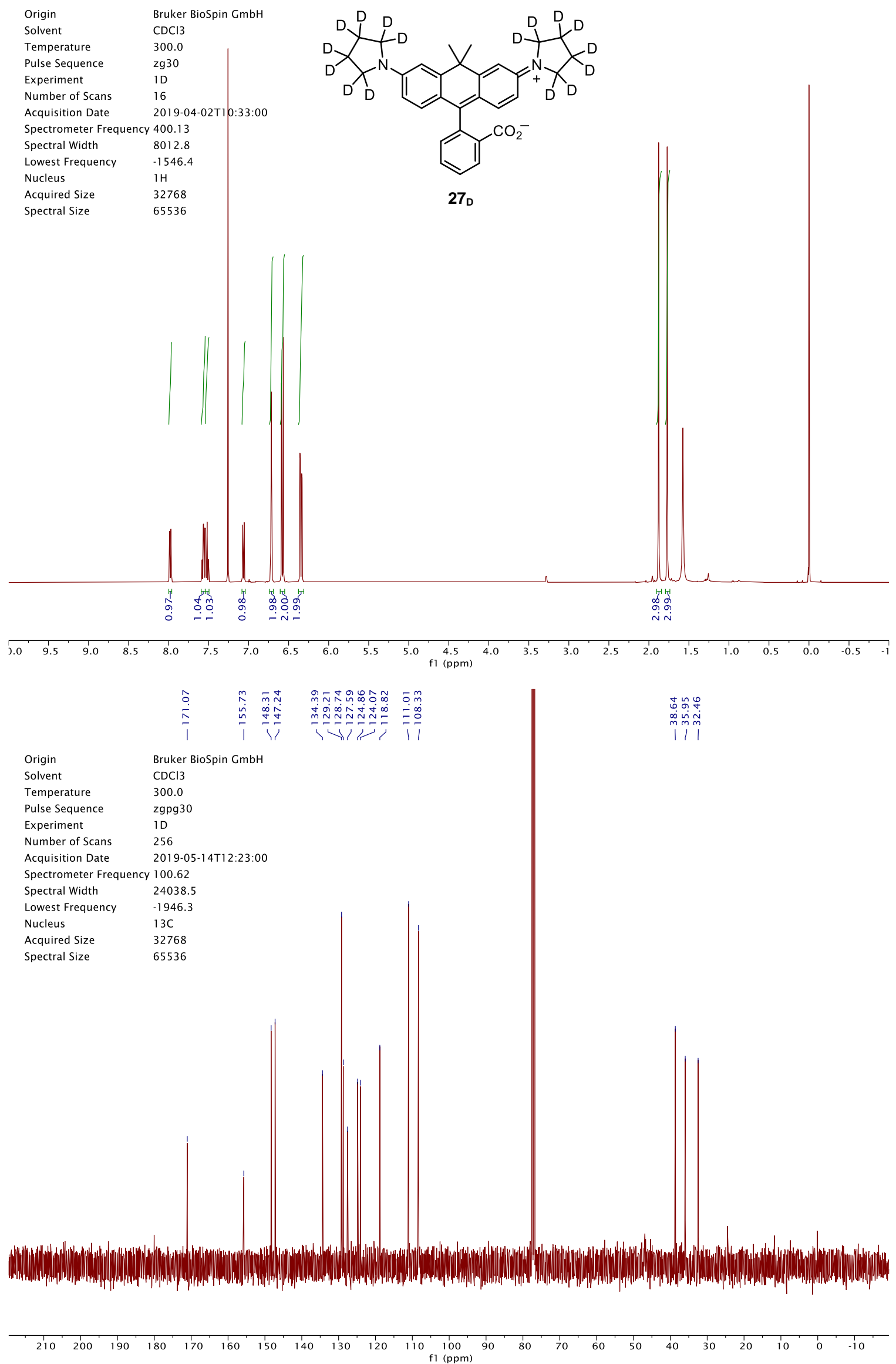


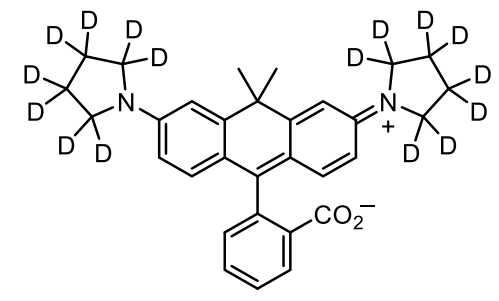

27D

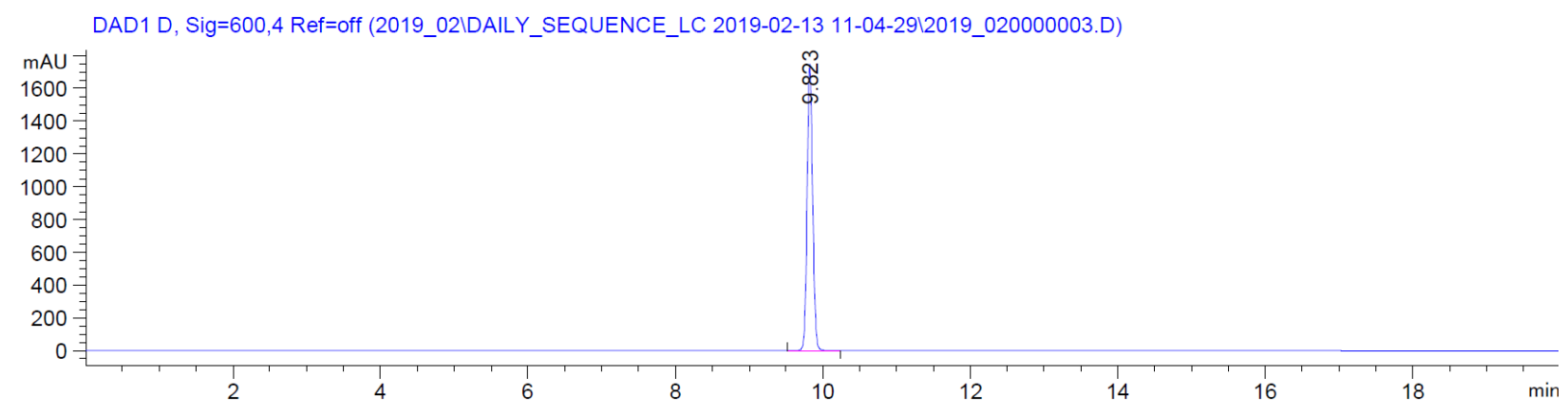

${ }^{*} M S D 2$ SPC, time=9.790:9.953 of C:ICHEM32I1IDATAI2019_02IDAILY_SEQUENCE_LC 2019-02-13 11-04-2912019_020000003.D ES-AP

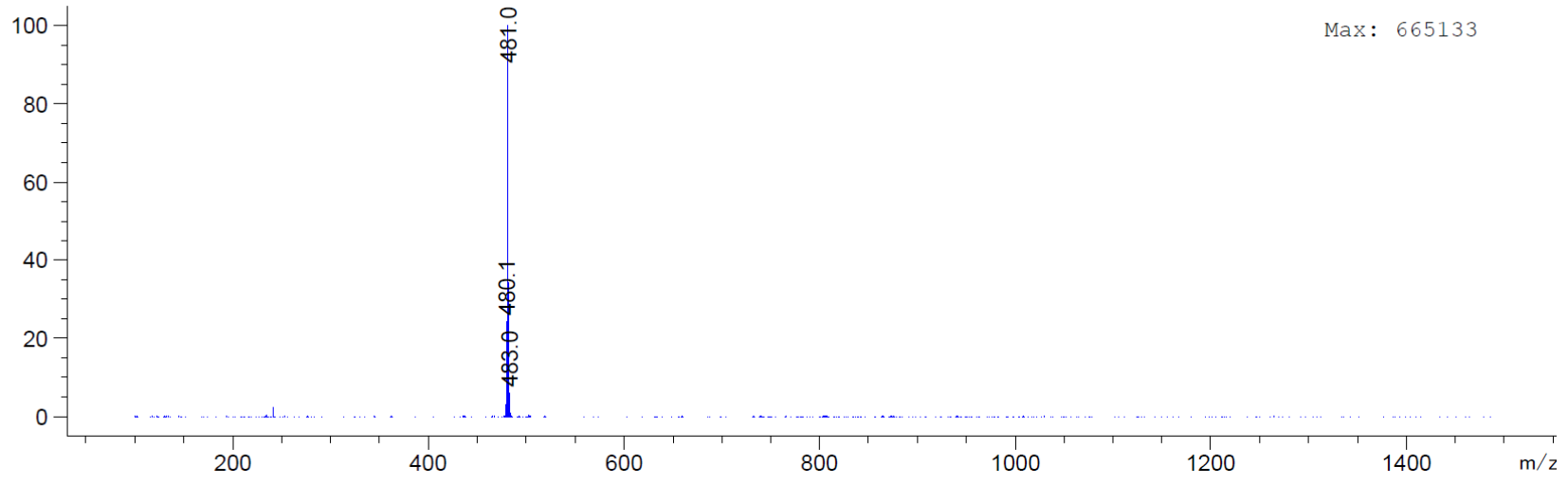



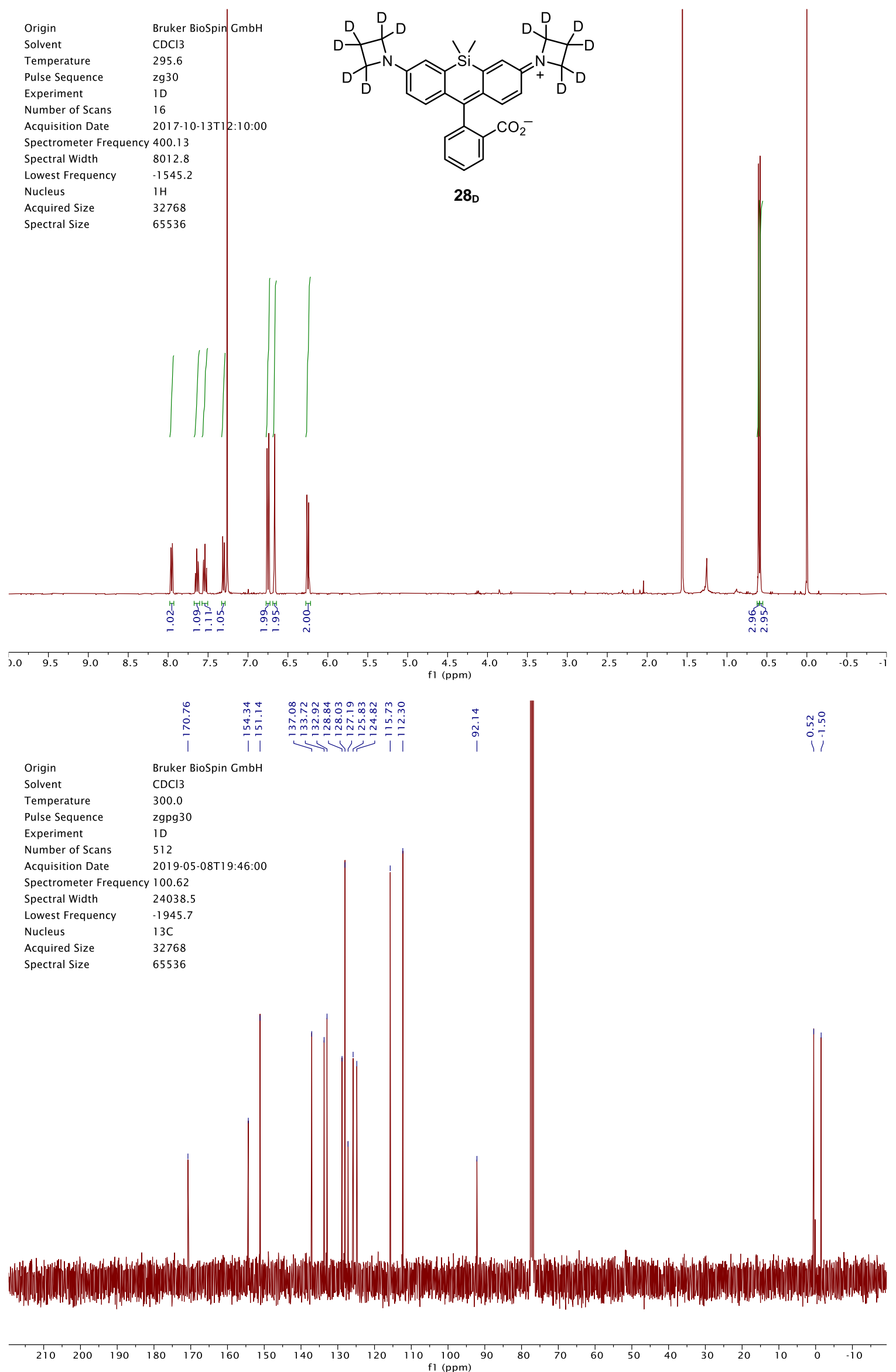


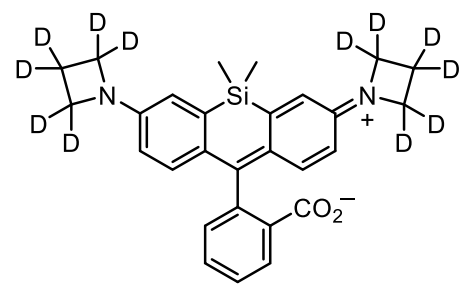

28 D

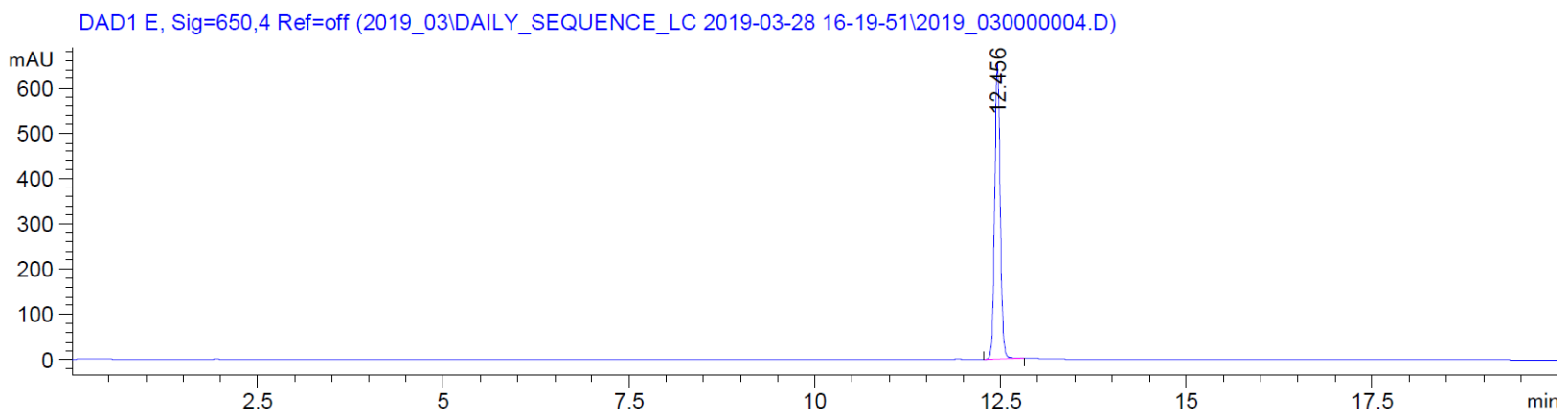

${ }^{*}$ MSD2 SPC, time=12.442:12.551 of C:ICHEM32I1IDATAI2019_03IDAILY_SEQUENCE_LC 2019-03-28 16-19-5112019_0300000004.D ES-

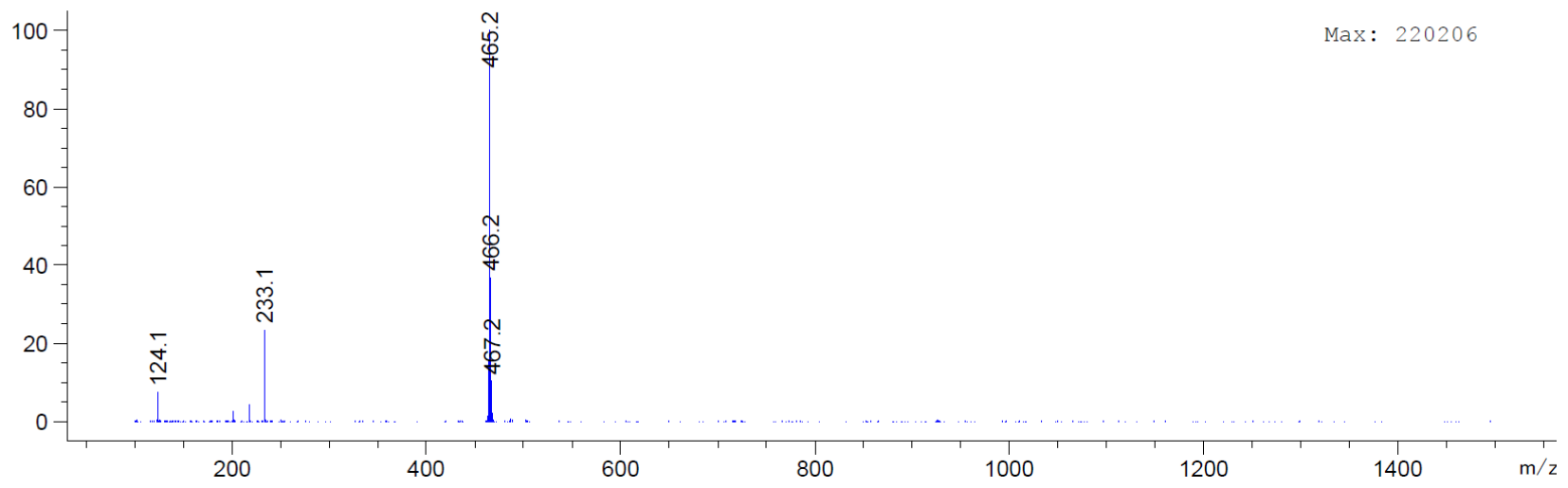



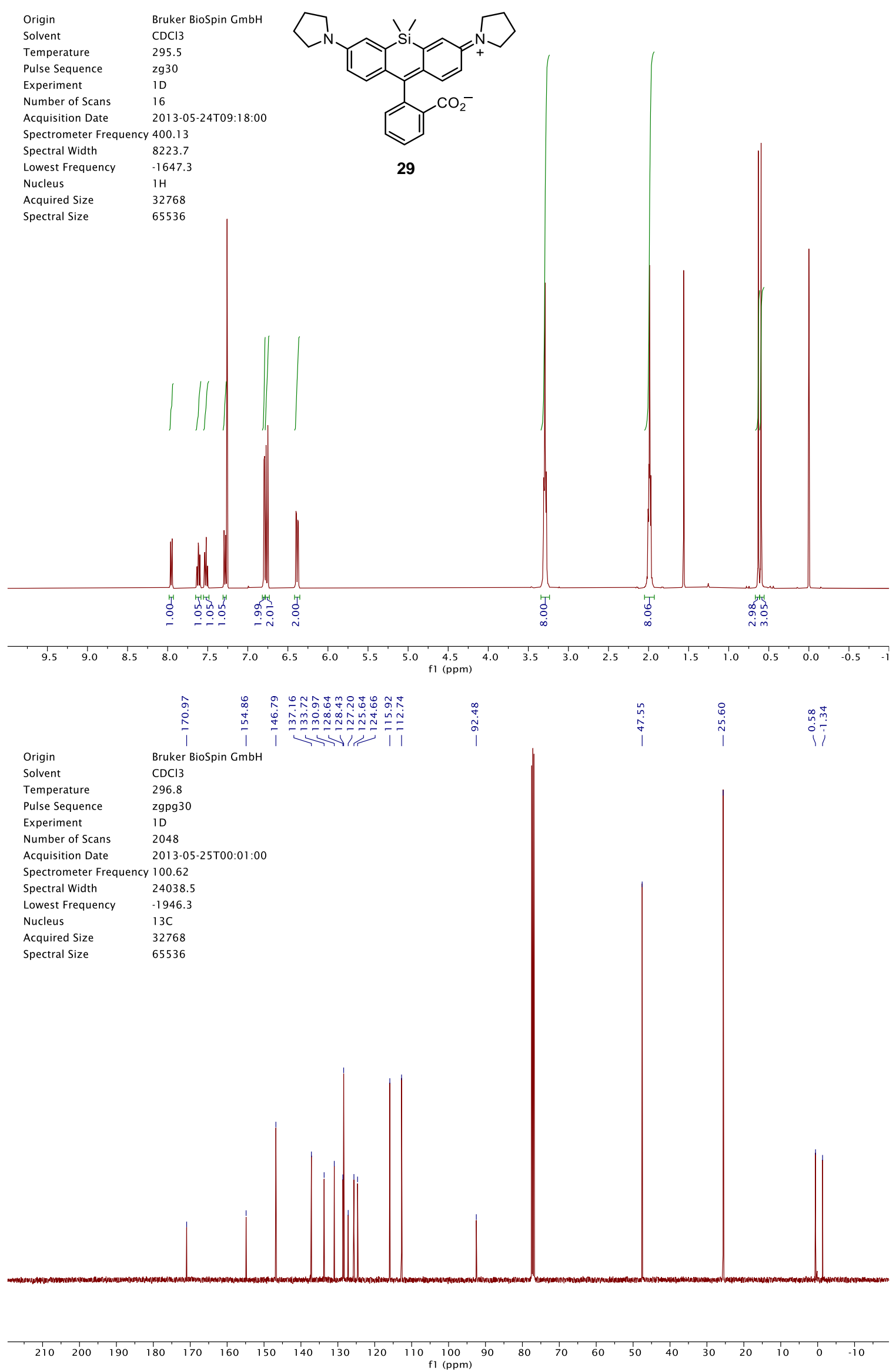


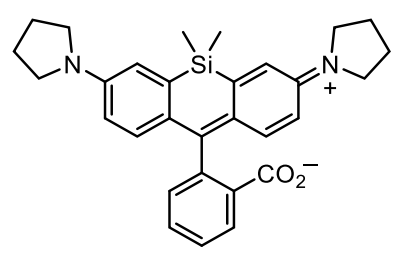

29

DAD1 E, Sig=650,16 Ref=off (2013 11IDAILYSQUENCE LC 2013-11-15 15-11-13/2013_11000001.D)

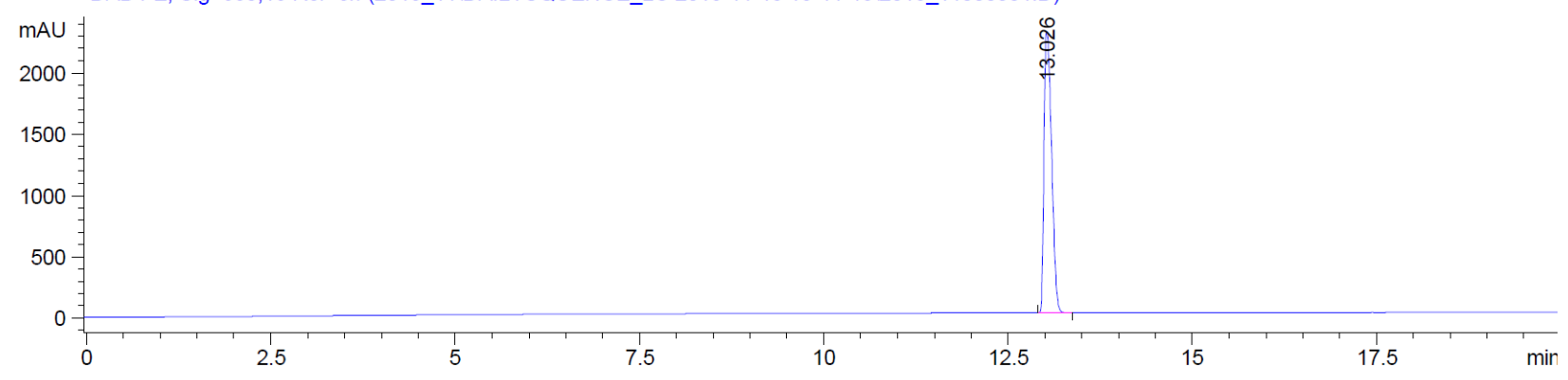

*MSD1 SPC, time=13.005:13.153 of C:ICHEM32I1IDATA|2013_11IDAILYSQUENCE_LC 2013-11-15 15-11-13|2013_11000001.D ES-API

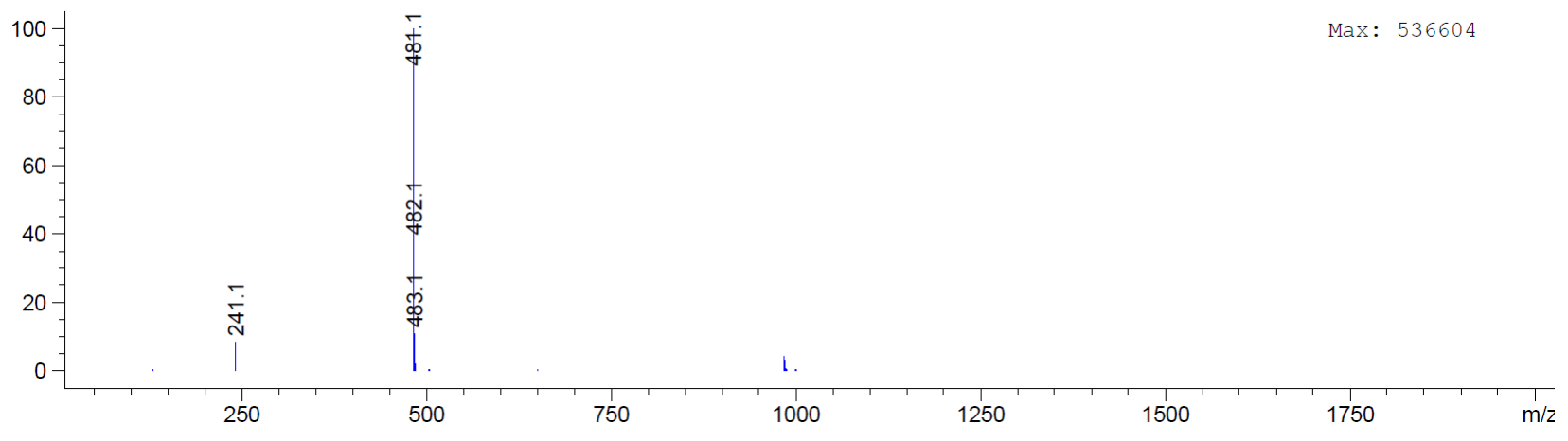



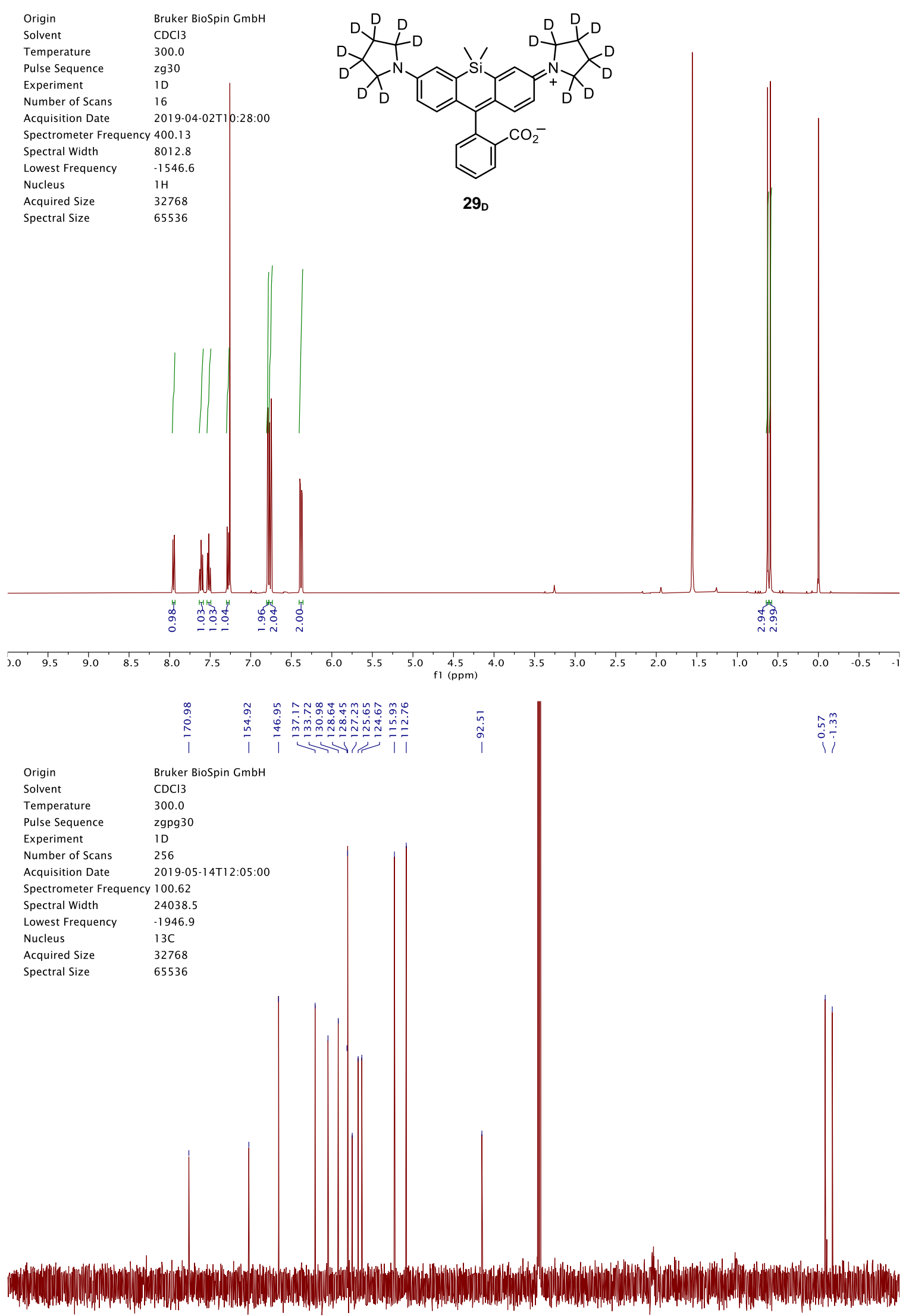

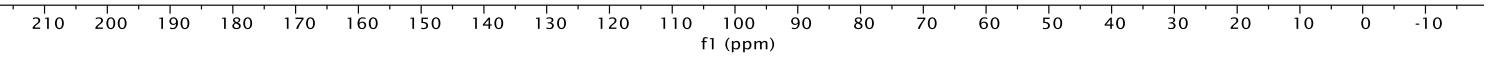




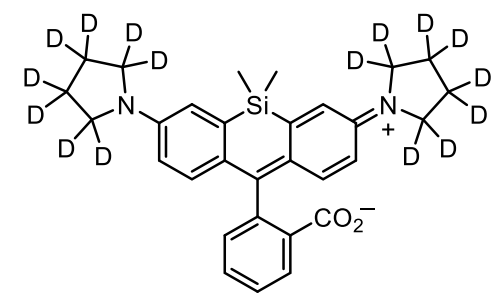

29D

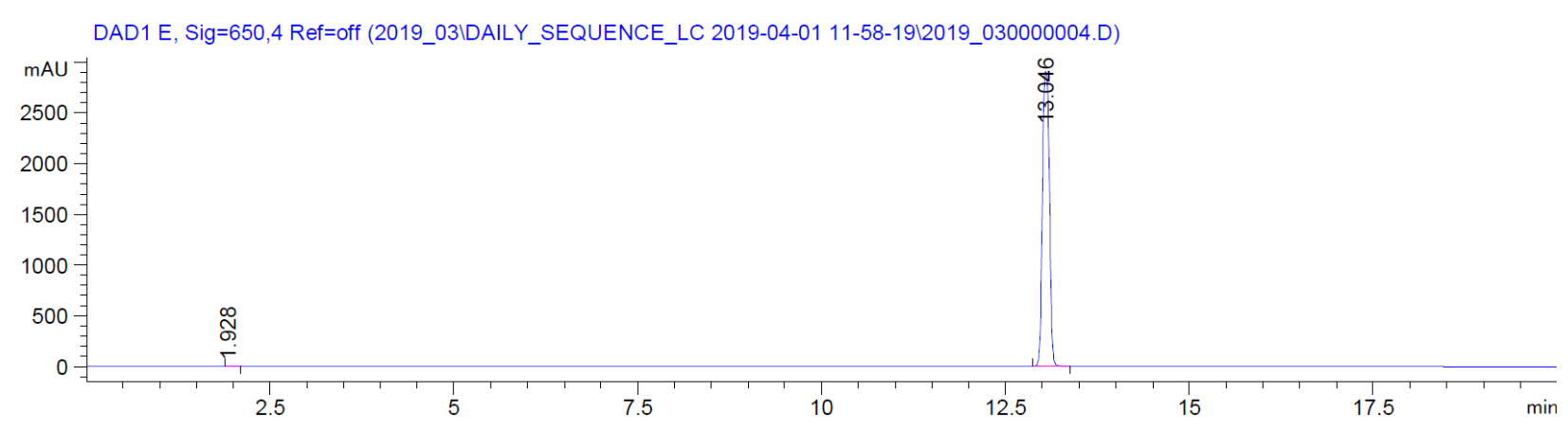

*MSD2 SPC, time=13.042:13.133 of C:ICHEM32I1IDATAI2019_03IDAILY_SEQUENCE_LC 2019-04-01 11-58-1912019_0300000004.D ES-

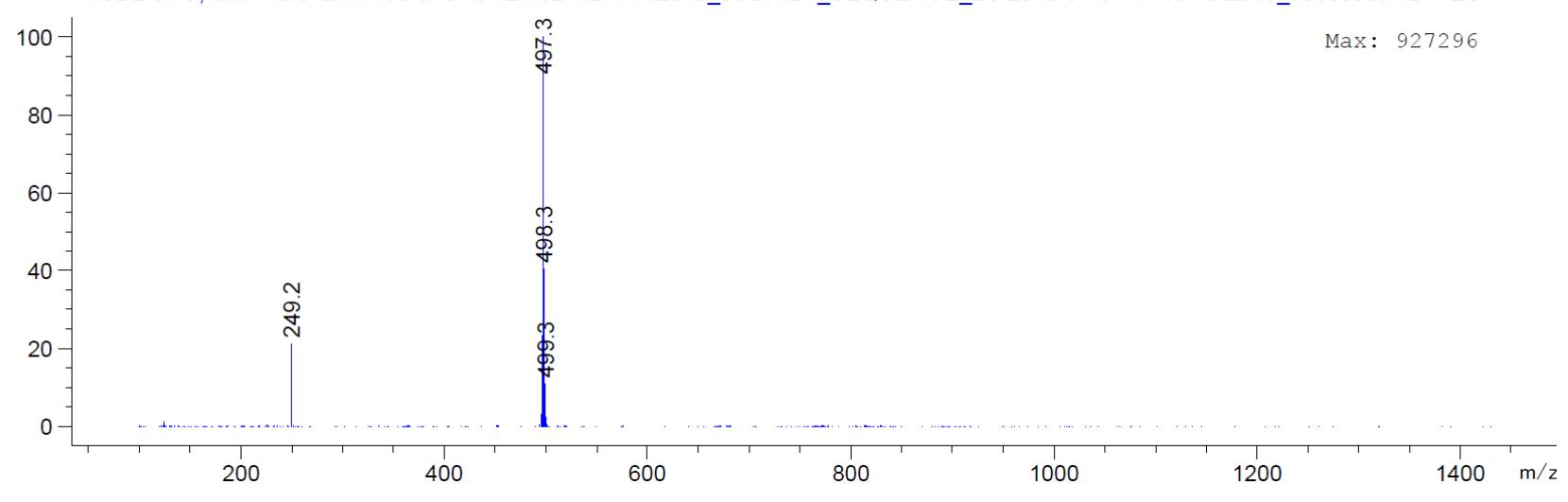



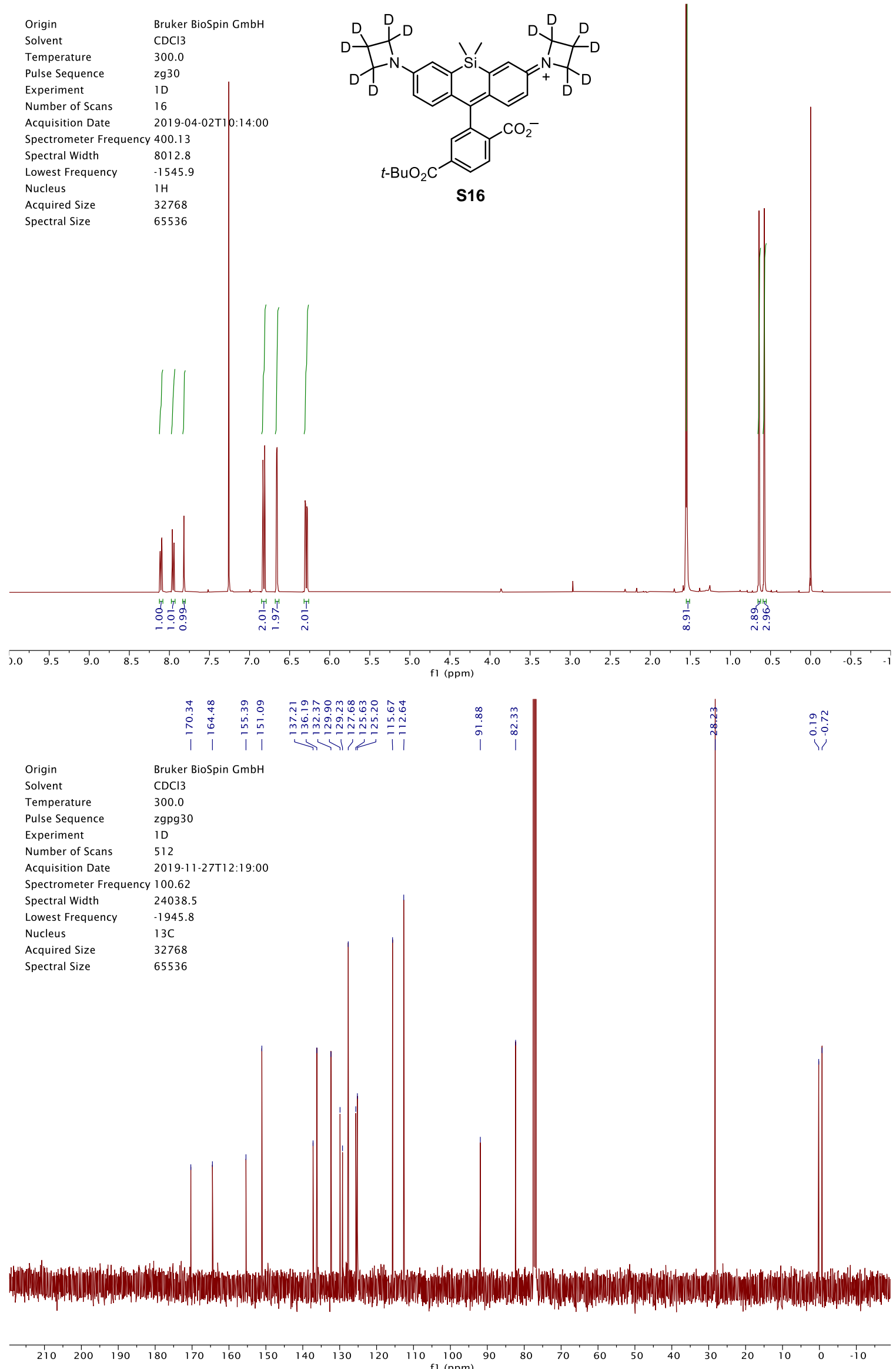


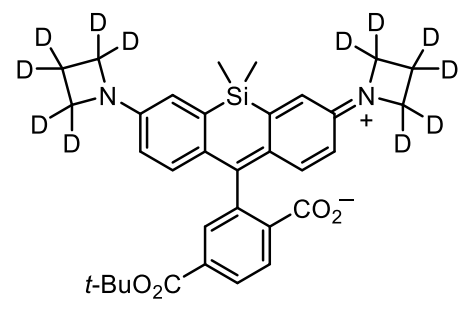

S16

DAD1 E, Sig=650,4 Ref=off (2019_03IDAILY_SEQUENCE_LC 2019-04-01 11-58-1912019_030000002.D)

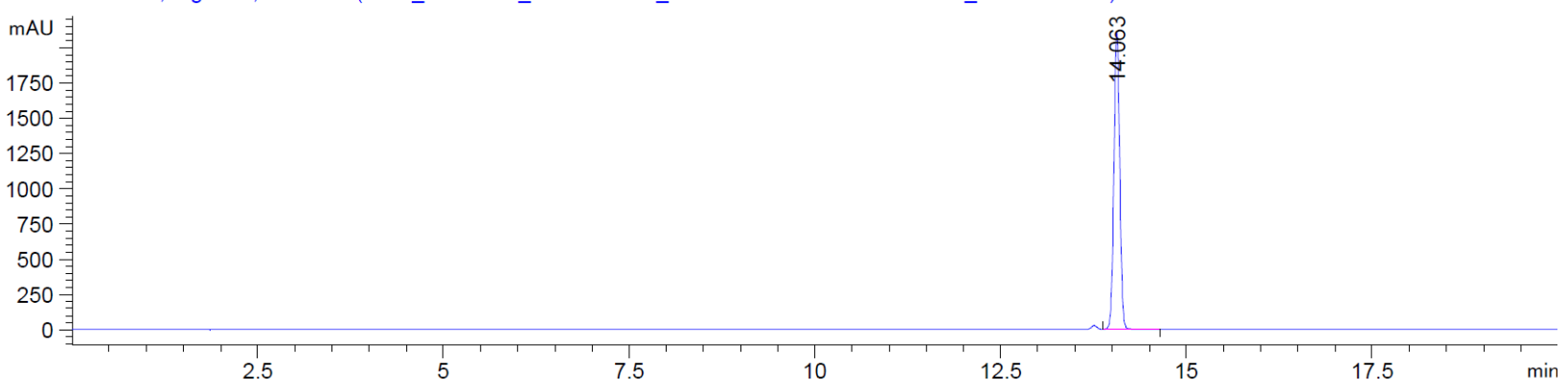

*MSD2 SPC, time=14.059:14.150 of C:ICHEM32I1IDATAI2019_03IDAILY_SEQUENCE_LC 2019-04-01 11-58-1912019_030000002.D ES-

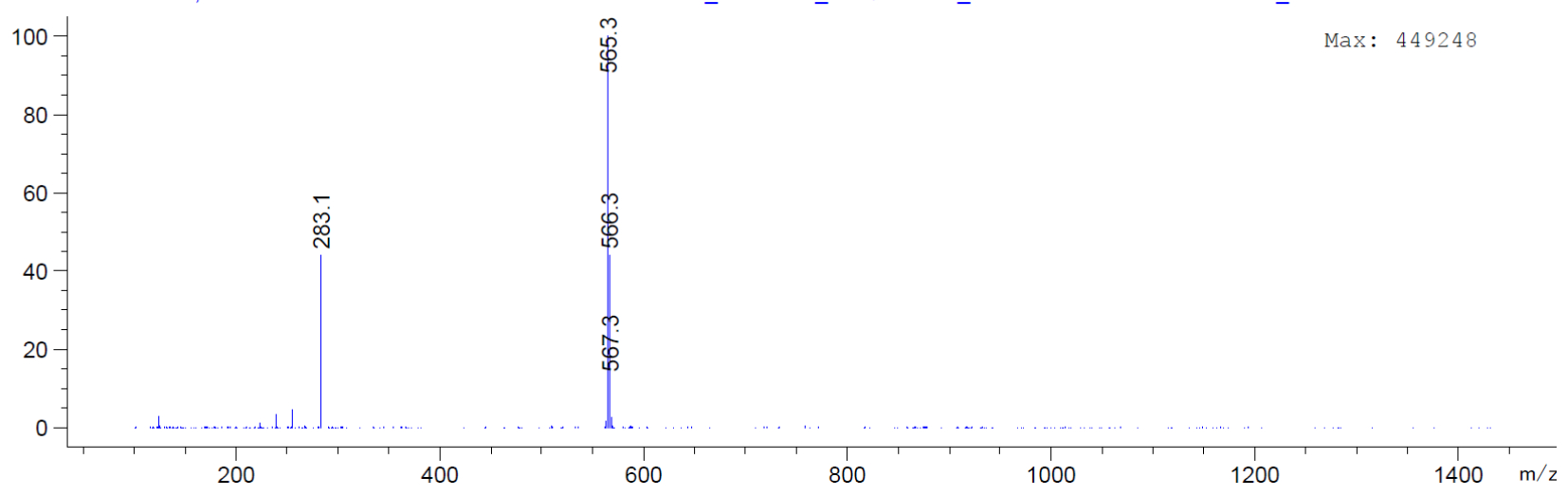



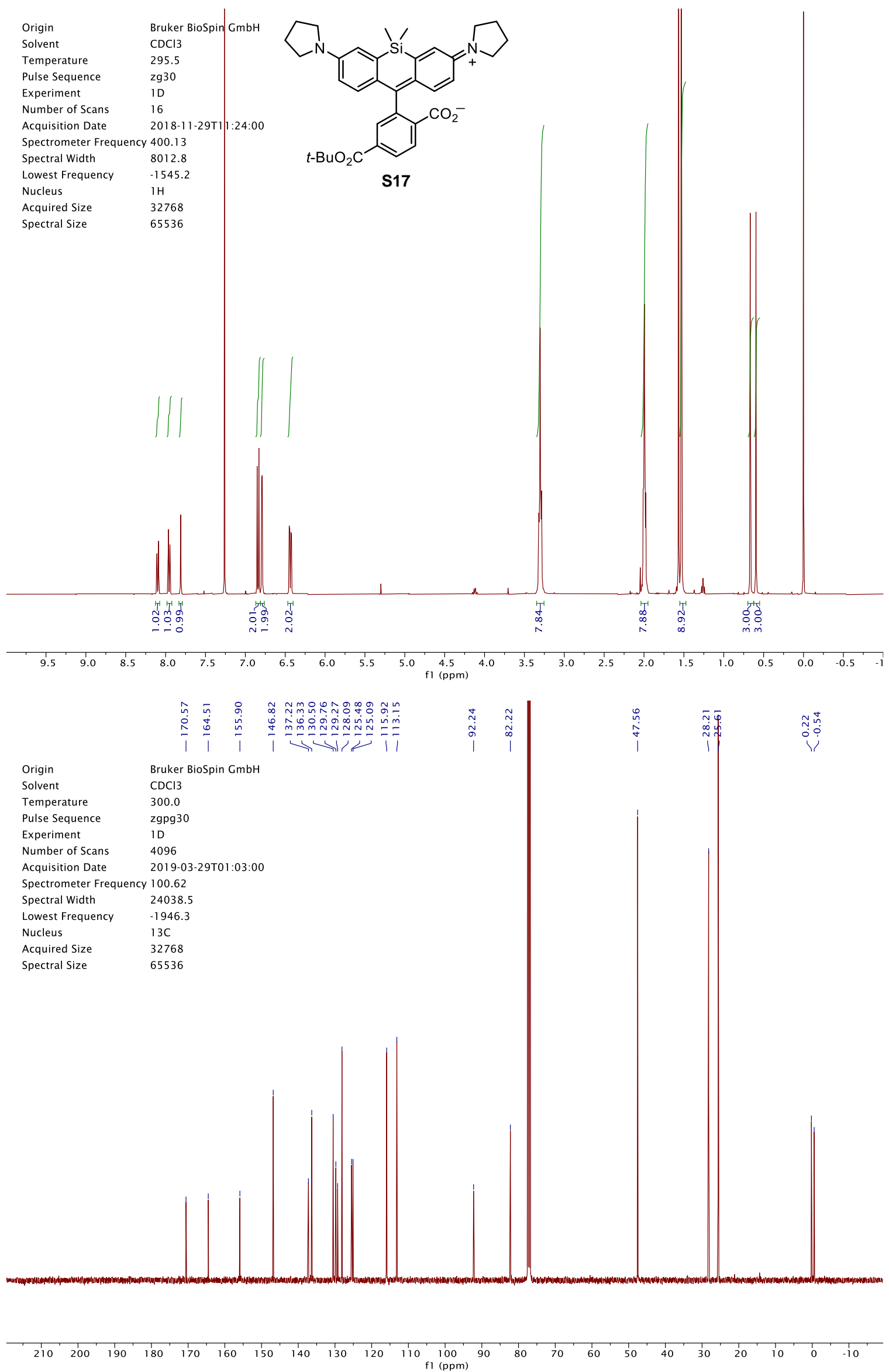

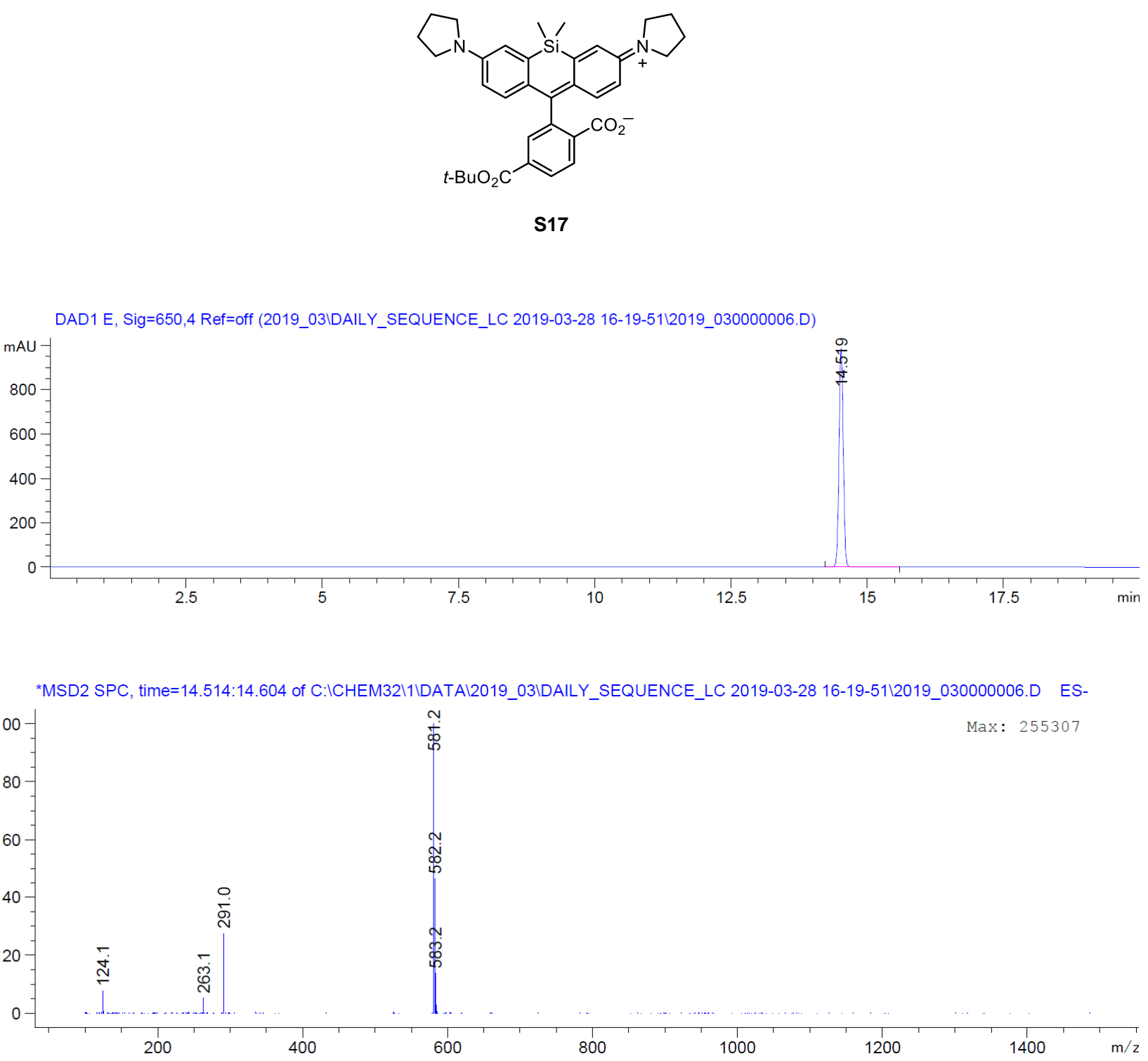

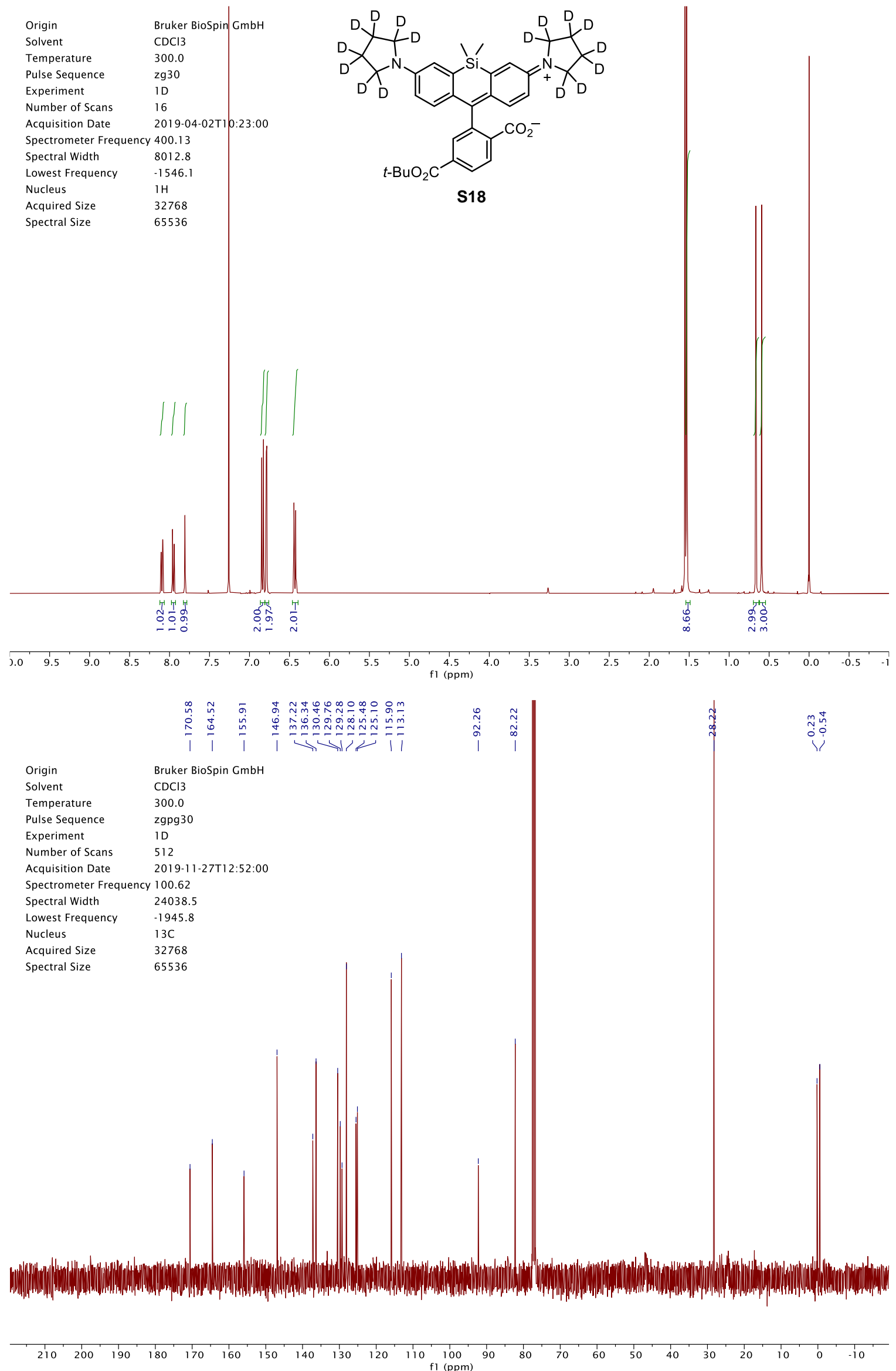

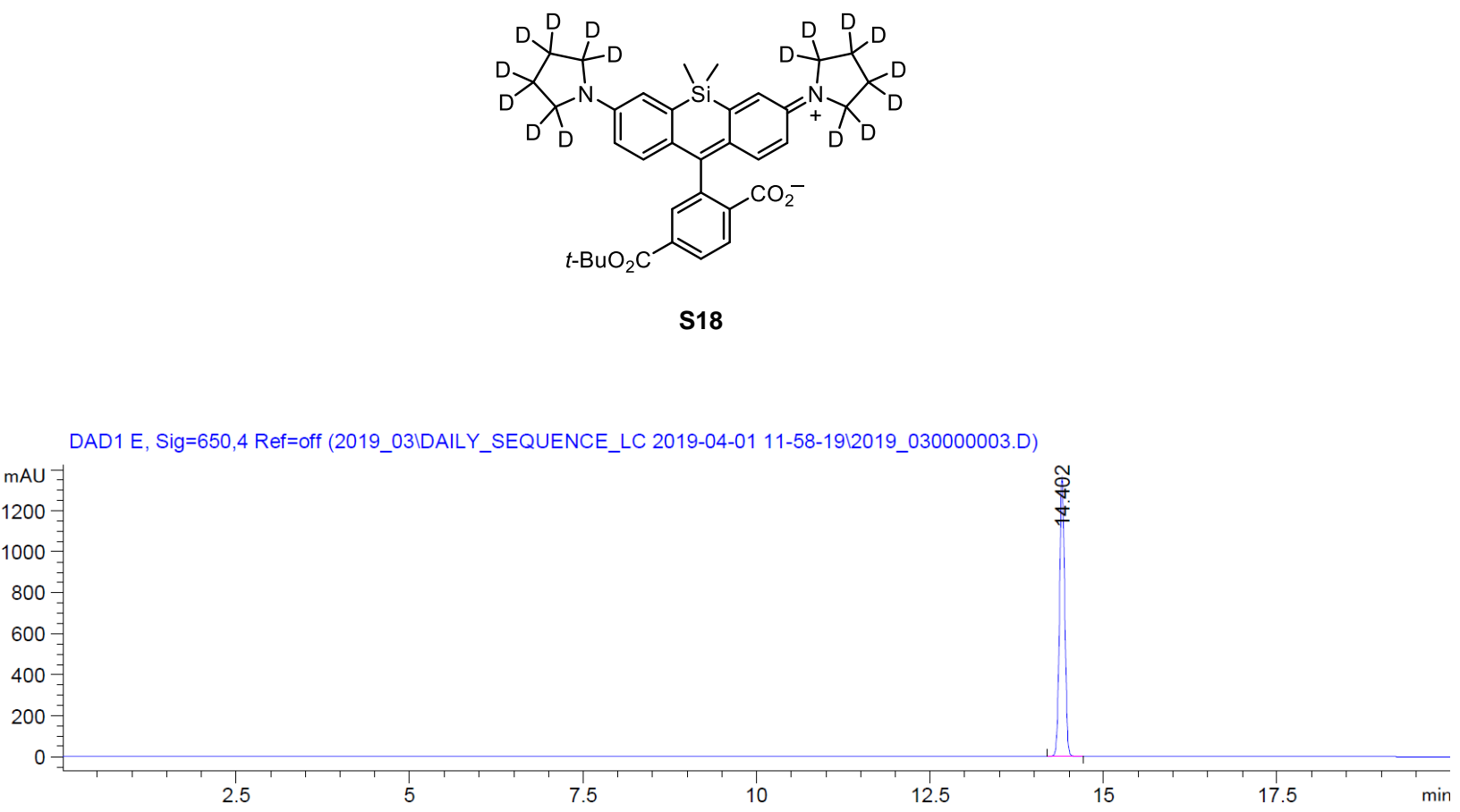

${ }^{*}$ MSD2 SPC, time=14.404:14.495 of C:ICHEM32I1IDATAI2019_03IDAILY_SEQUENCE_LC 2019-04-01 11-58-1912019_0300000003.D ES-

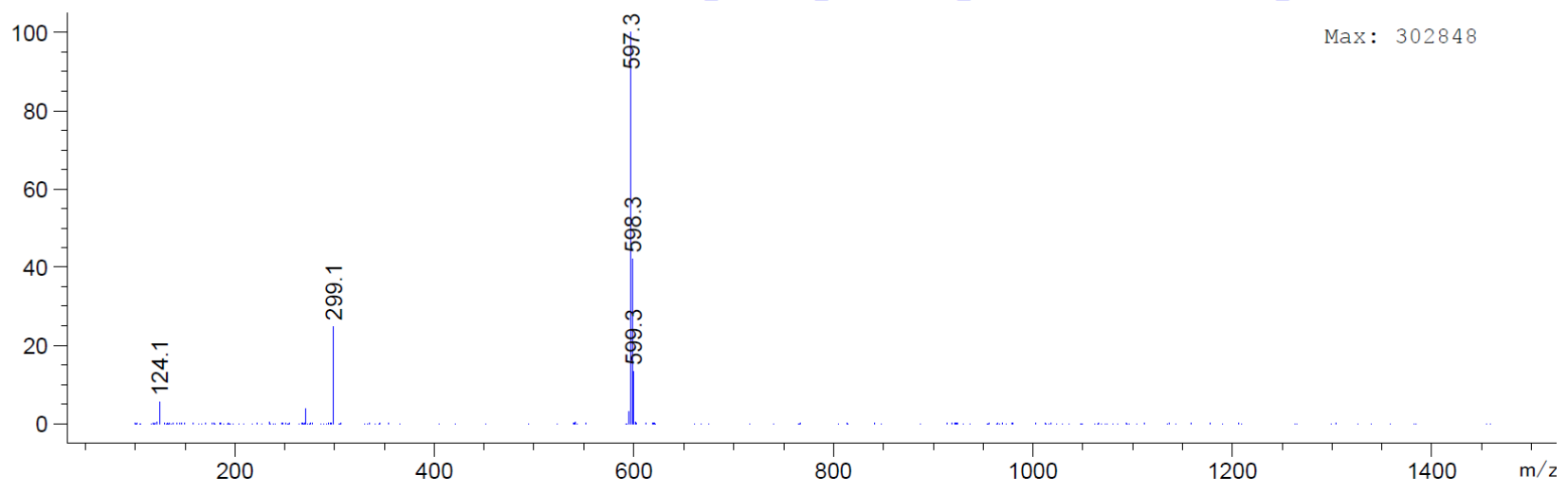



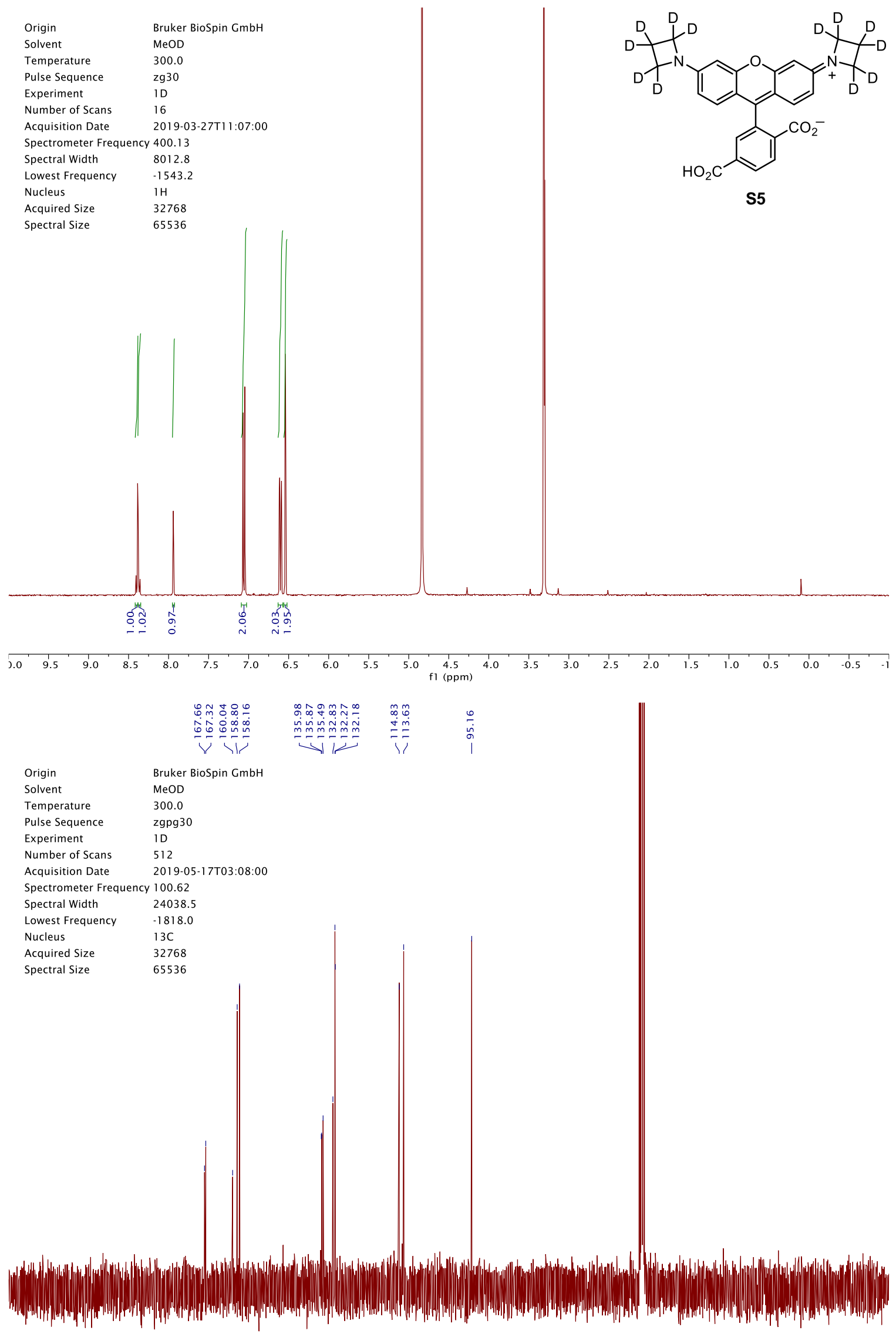

$\begin{array}{llllllllllllllllllllllll}1 & 1 \\ 20 & 210 & 200 & 190 & 180 & 170 & 160 & 150 & 140 & 130 & 120 & 110 & 100 & 90 & 80 & 70 & 60 & 50 & 40 & 30 & 20 & 10 & 0 & -10\end{array}$ 


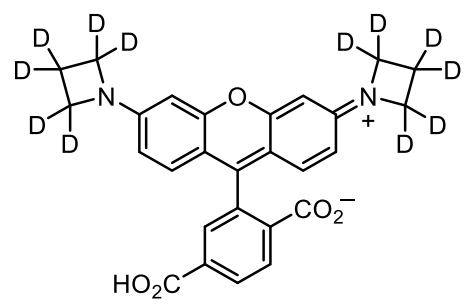

S5

DAD1 C, Sig=550,4 Ref=off (2019_03IDAILY_SEQUENCE_LC 2019-03-26 11-59-3912019_030000005.D)

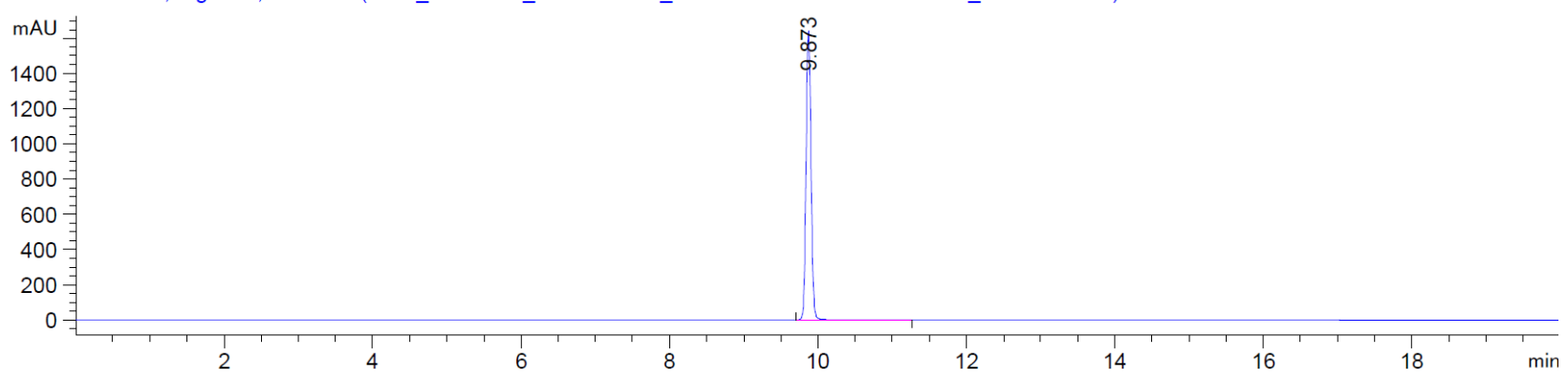

${ }^{*}$ MSD2 SPC, time=9.881:9.953 of C:ICHEM3211IDATAI2019_03IDAILY_SEQUENCE_LC 2019-03-26 11-59-3912019_030000005.D ES-AP

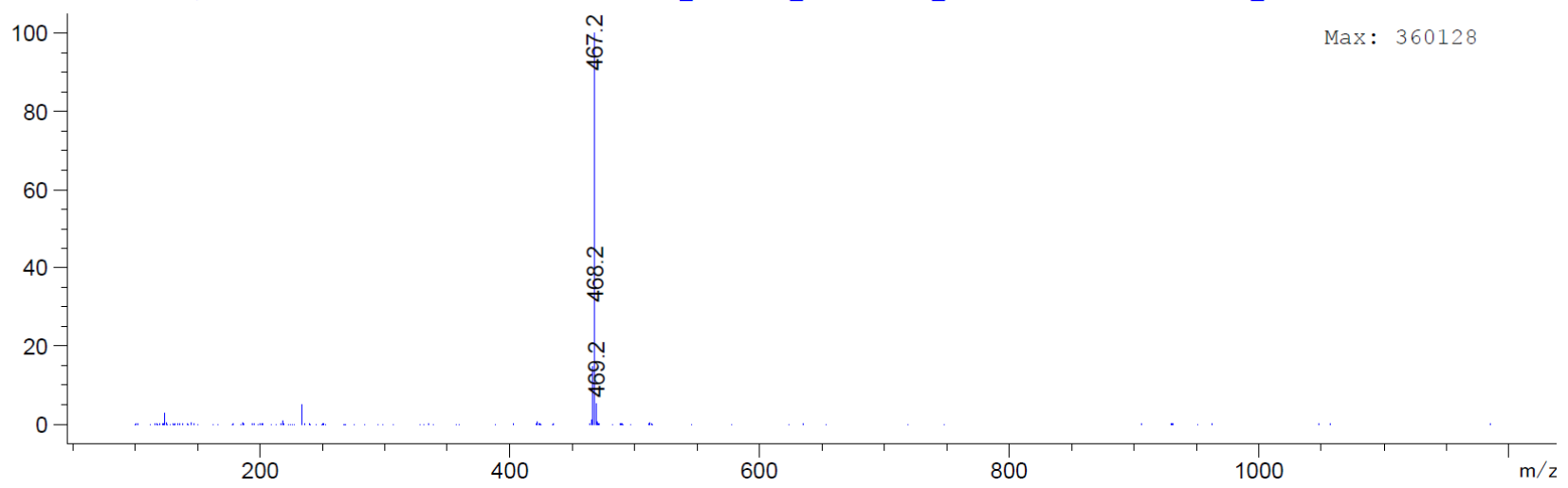



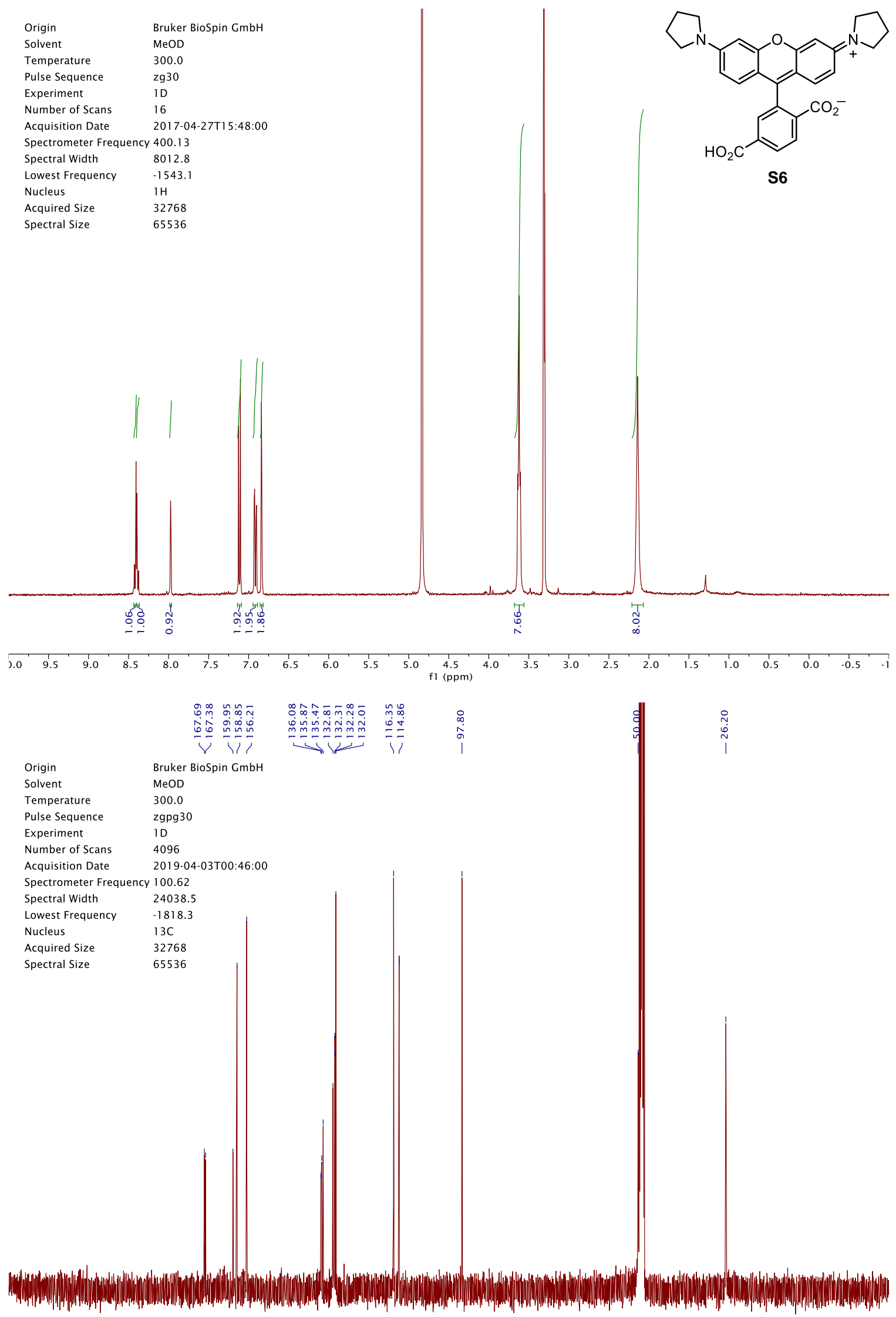

$\begin{array}{lllllllllllllllllllllllllllllllll}1 & 20 & 210 & 200 & 190 & 180 & 170 & 160 & 150 & 140 & 130 & 120 & 110 & 100 & 90 & 80 & 70 & 60 & 50 & 40 & 30 & 20 & 10 & 0 & -10\end{array}$ 


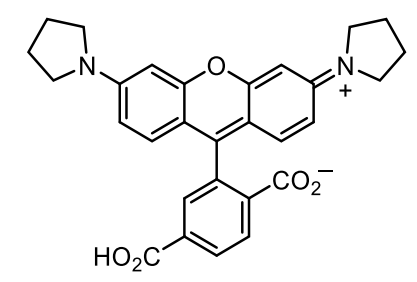

S6

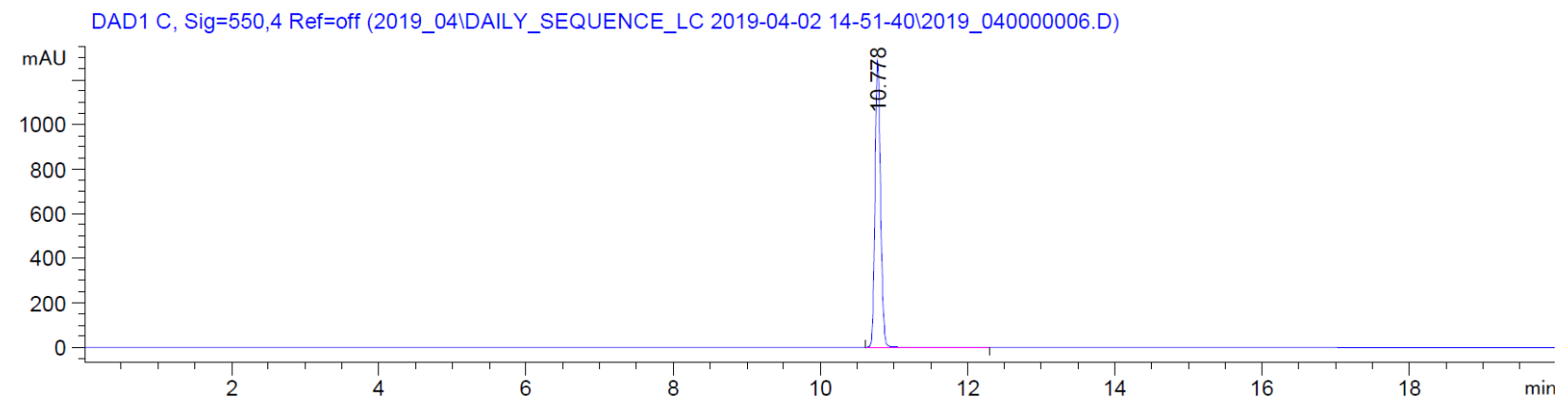

*MSD2 SPC, time=10.789:10.880 of C:ICHEM32I1IDATAI2019_04IDAILY_SEQUENCE_LC 2019-04-02 14-51-4012019_040000006.D ES-

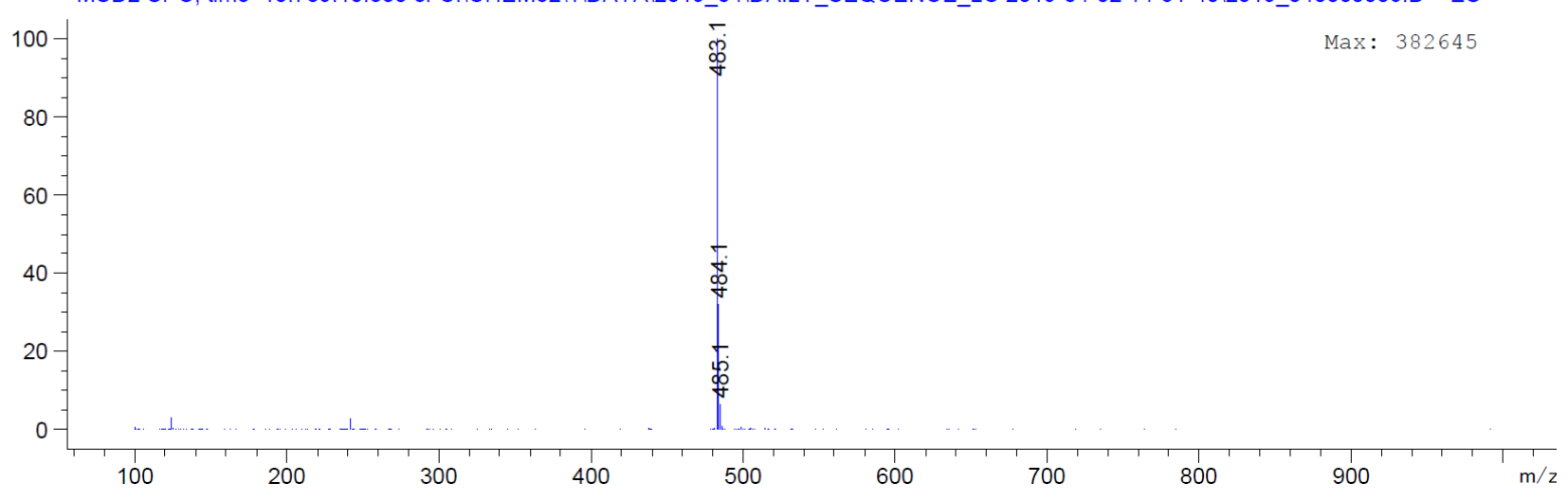



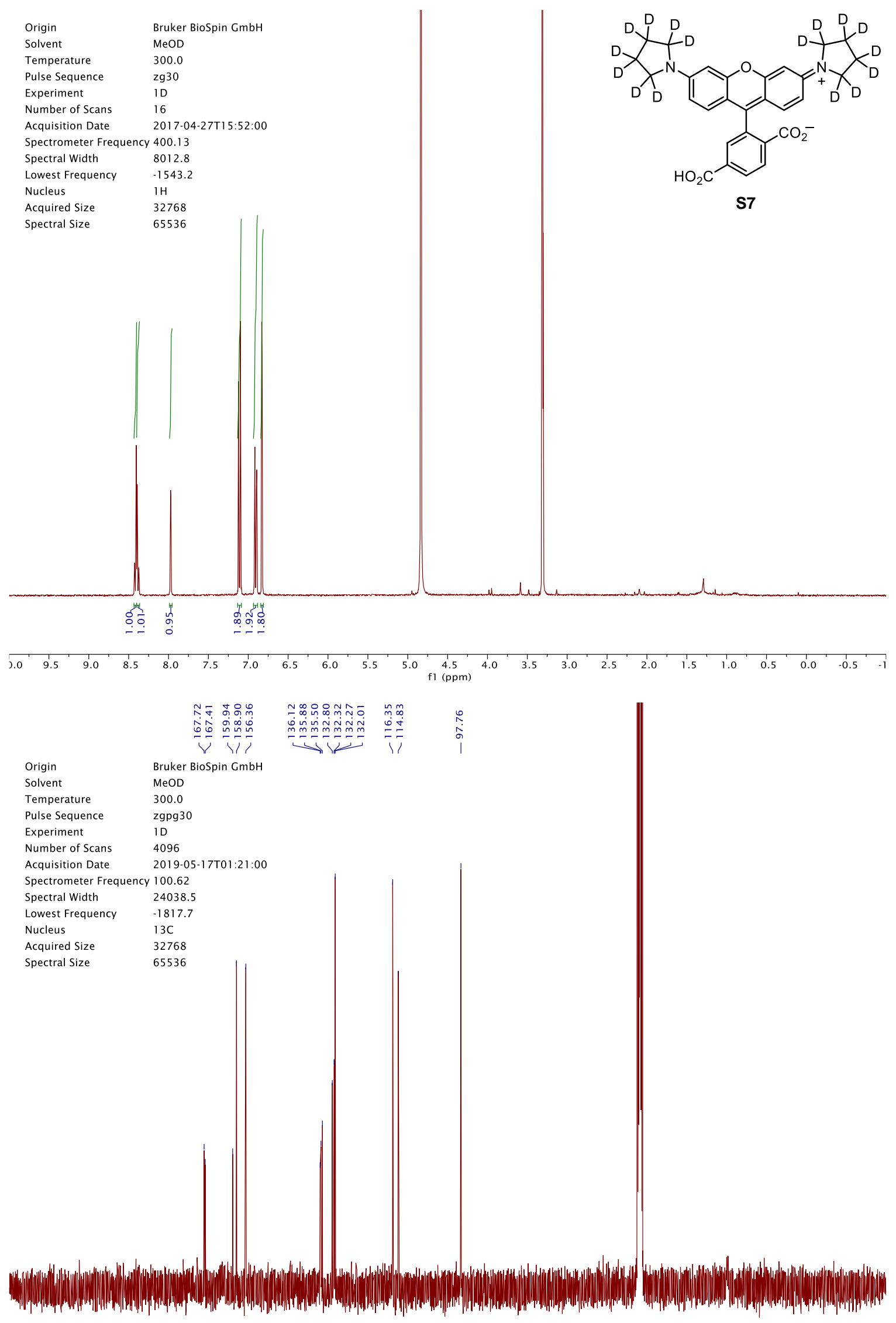

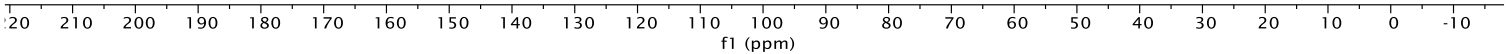




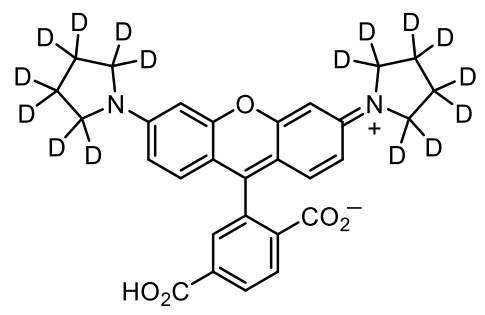

S7

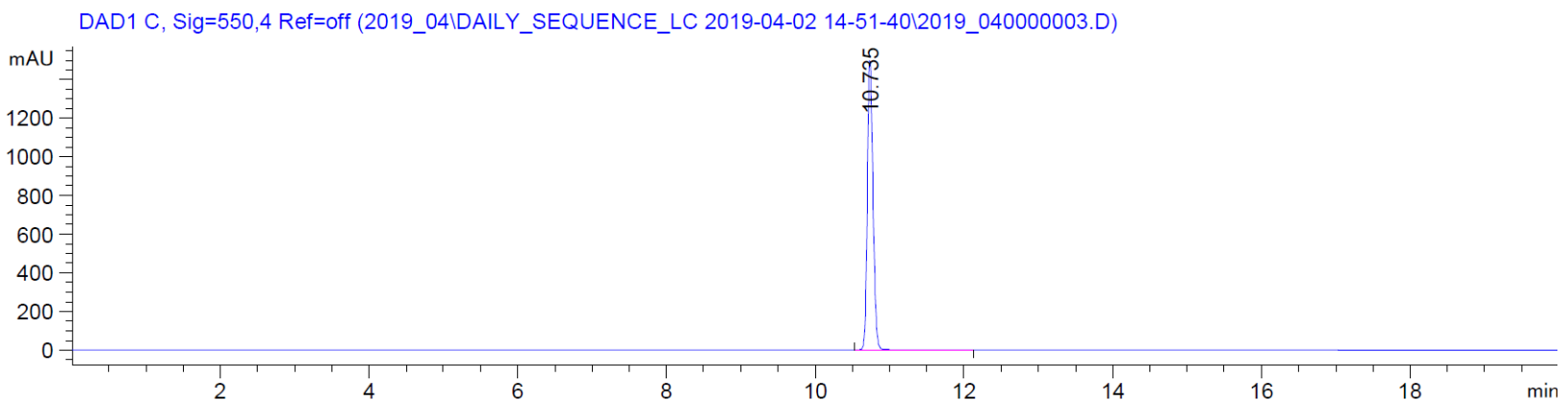

*MSD2 SPC, time=10.734:10.825 of C:ICHEM32I1IDATAI2019_04IDAILY_SEQUENCE_LC 2019-04-02 14-51-4012019_0400000003.D ES-

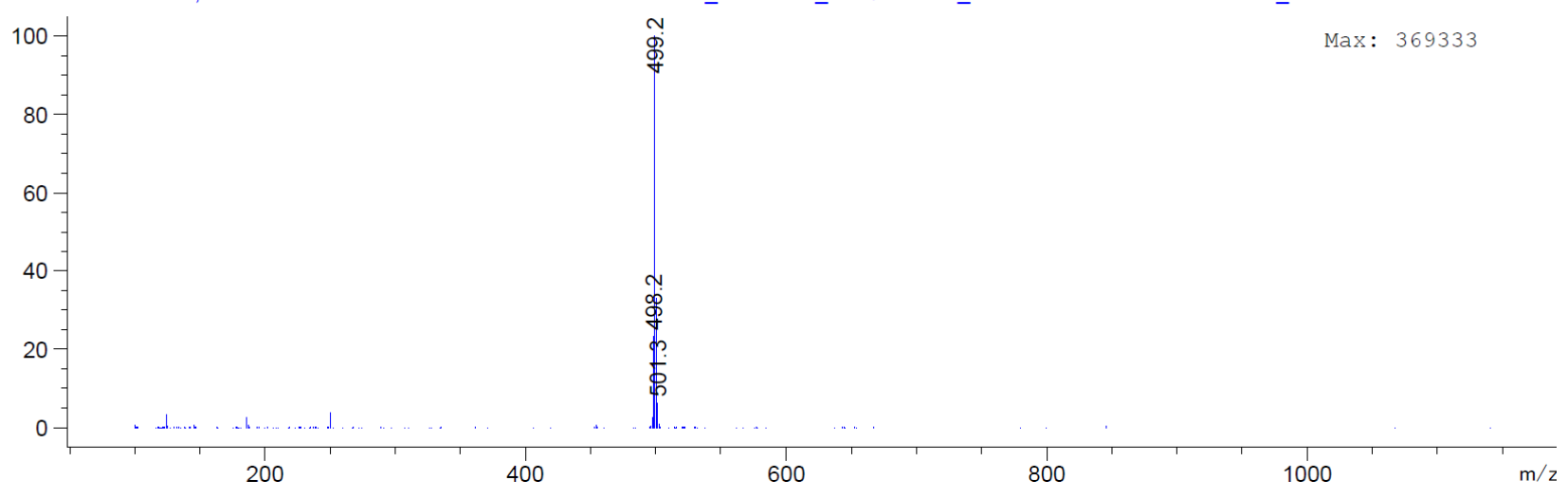




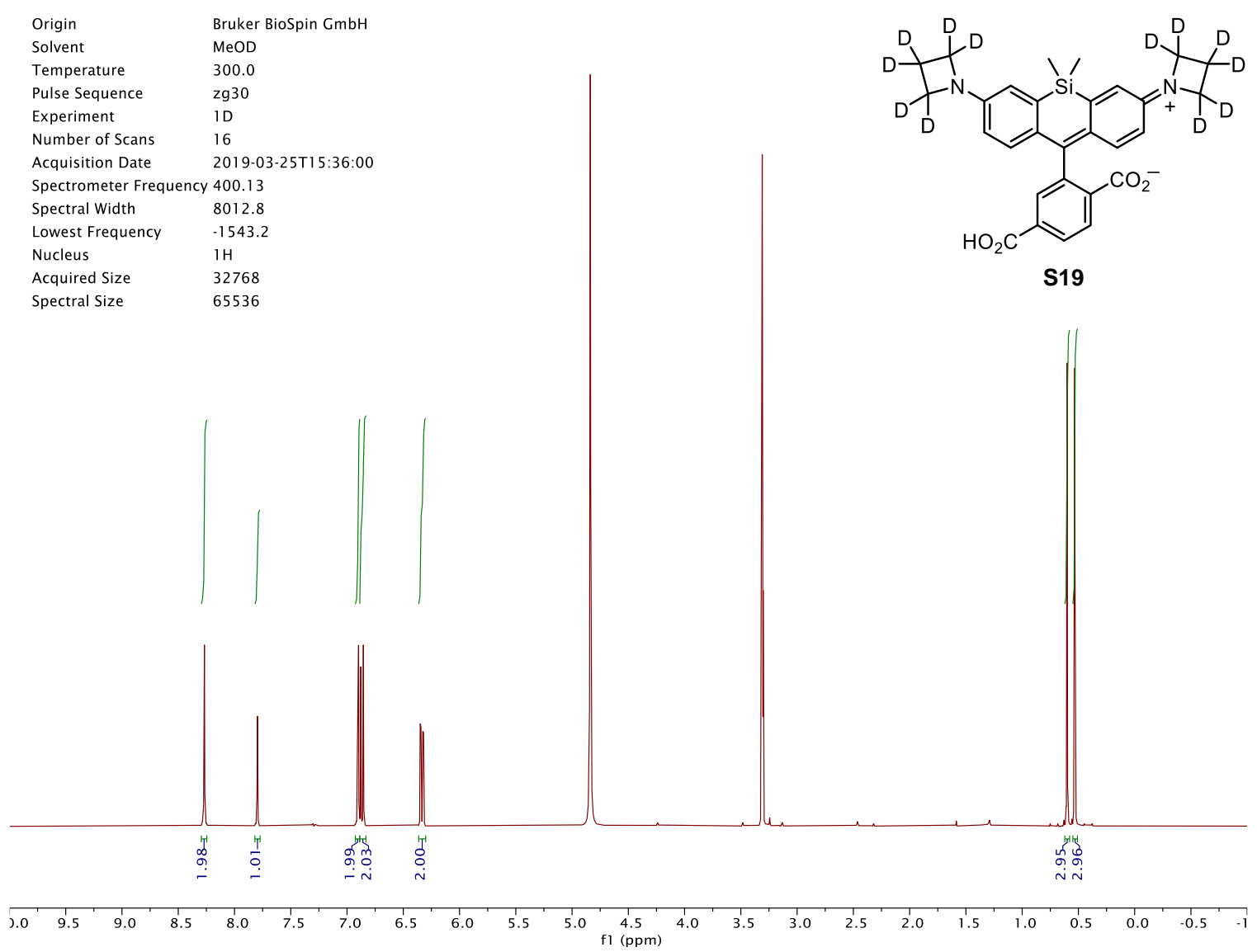

DAD1 E, Sig=650,4 Ref=off (2019_03IDAILY_SEQUENCE_LC 2019-03-25 15-17-1712019_030000003.D)

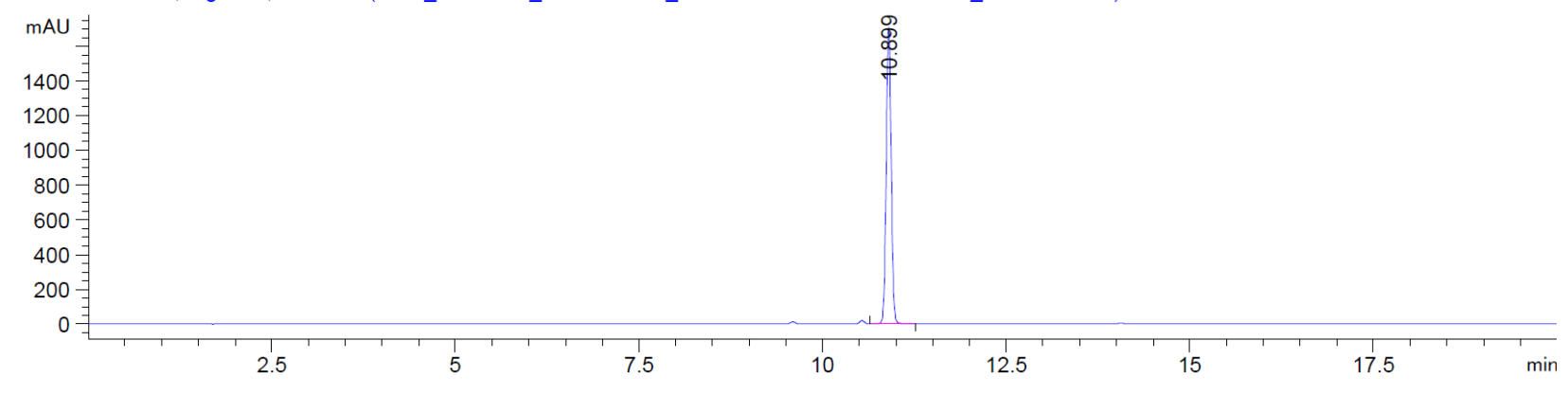

${ }^{*}$ MSD2 SPC, time=10.898:10.989 of C:ICHEM32I1IDATAI2019_03IDAILY_SEQUENCE_LC 2019-03-25 15-17-17/2019_030000003.D ES-

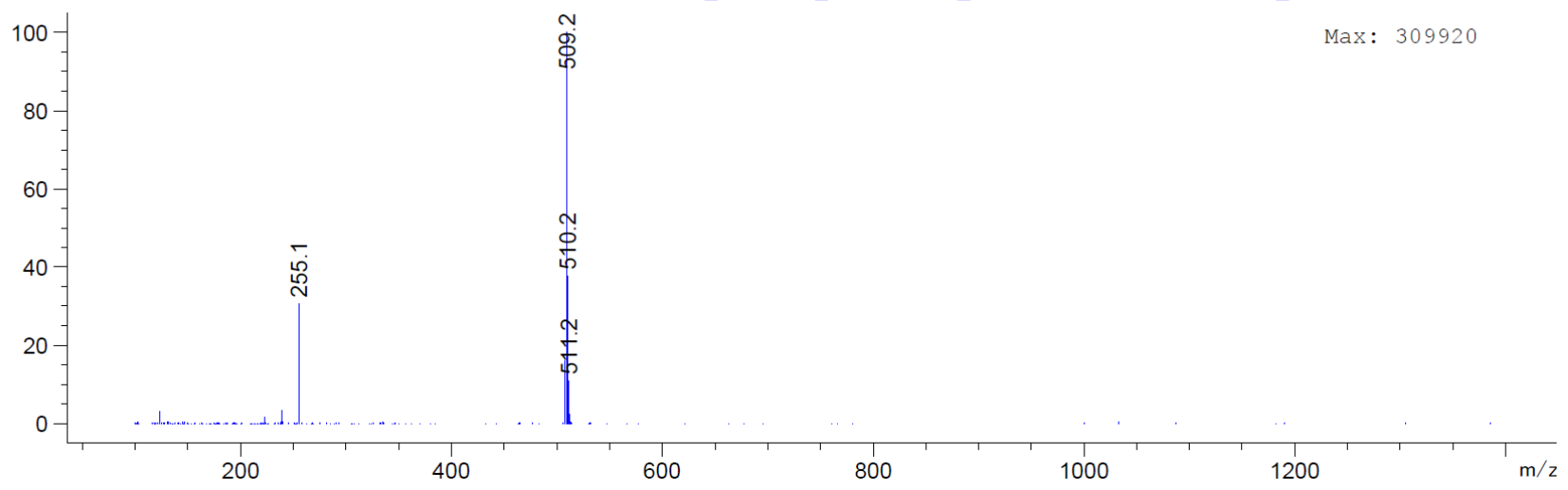



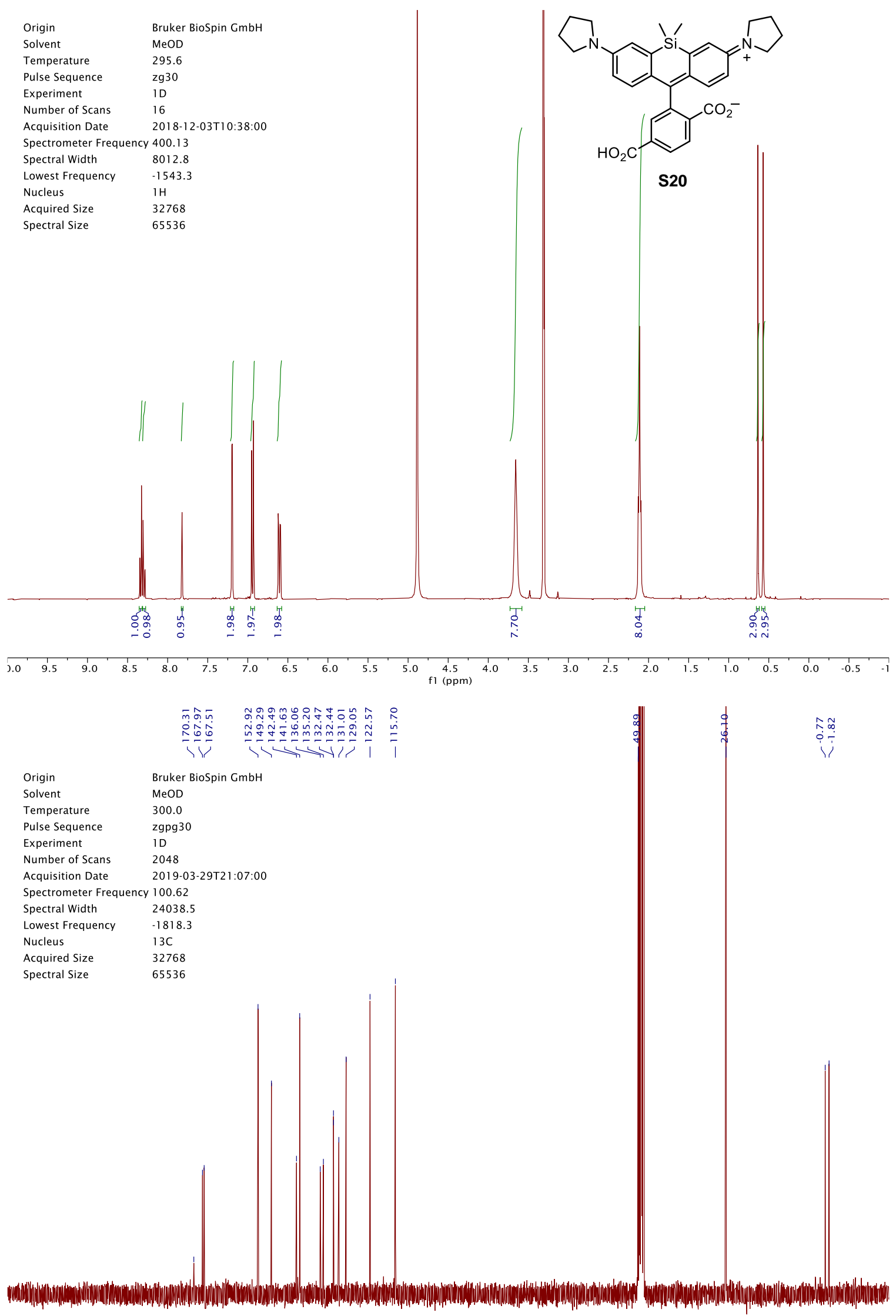

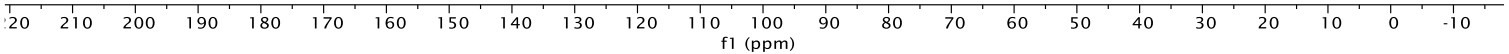




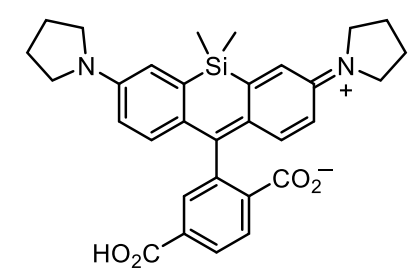

S20

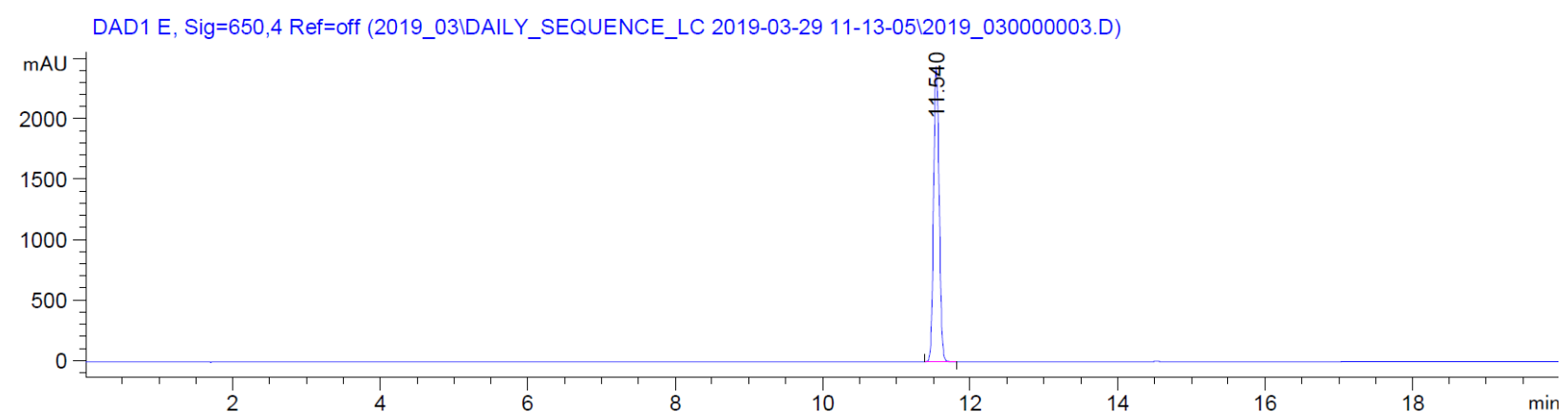

${ }^{*}$ MSD2 SPC, time=11.537:11.628 of C:ICHEM32I1IDATAI2019_03IDAILY_SEQUENCE_LC 2019-03-29 11-13-0512019_030000003.D ES-

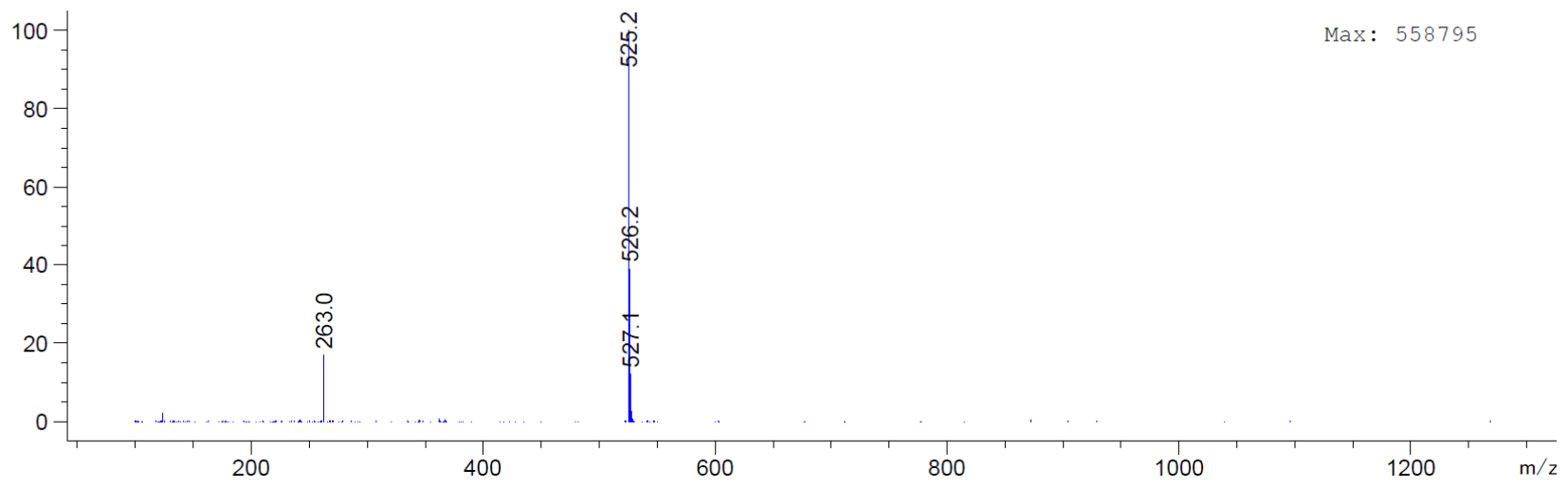



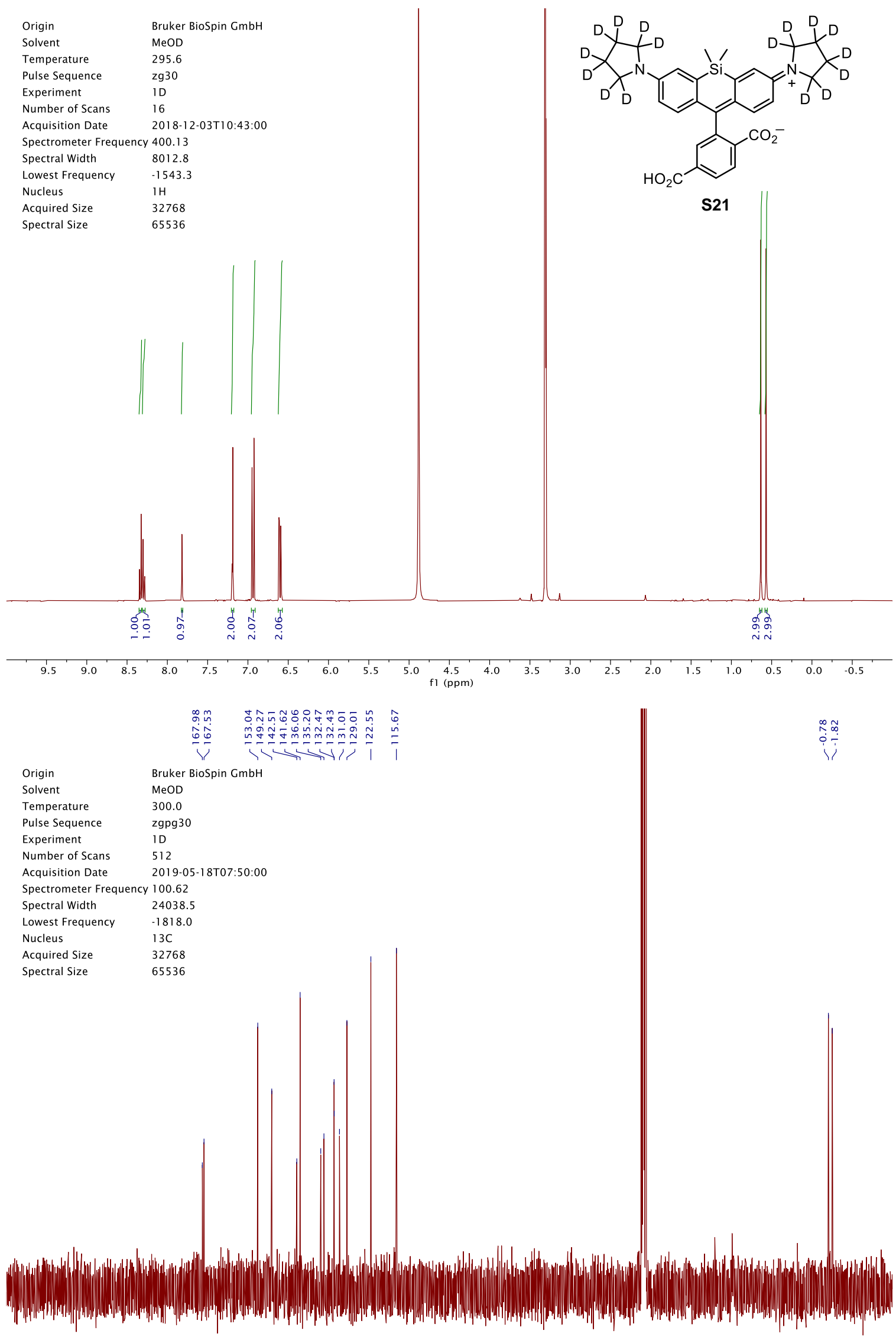

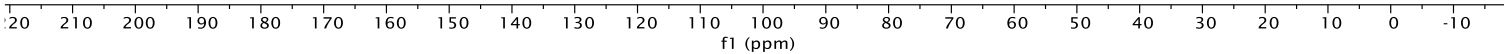




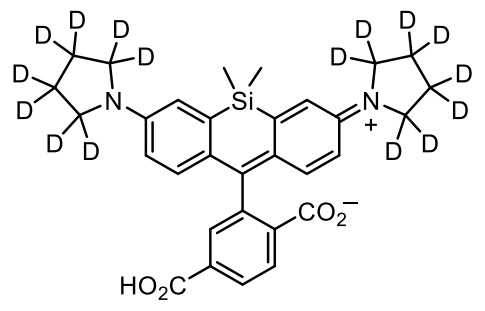

S21

DAD1 E, Sig=650,4 Ref=off (2019_03IDAILY_SEQUENCE_LC 2019-03-29 11-13-0512019_030000004.D)

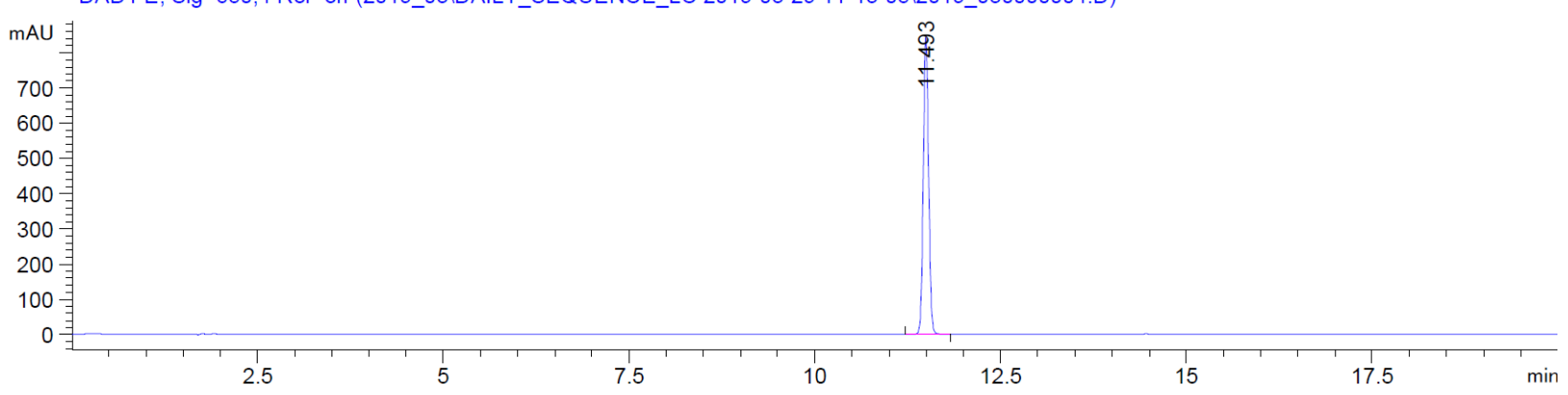

${ }^{*}$ MSD2 SPC, time=11.498:11.588 of C:ICHEM32I1IDATAI2019_03IDAILY_SEQUENCE_LC 2019-03-29 11-13-05।2019_030000004.D ES-

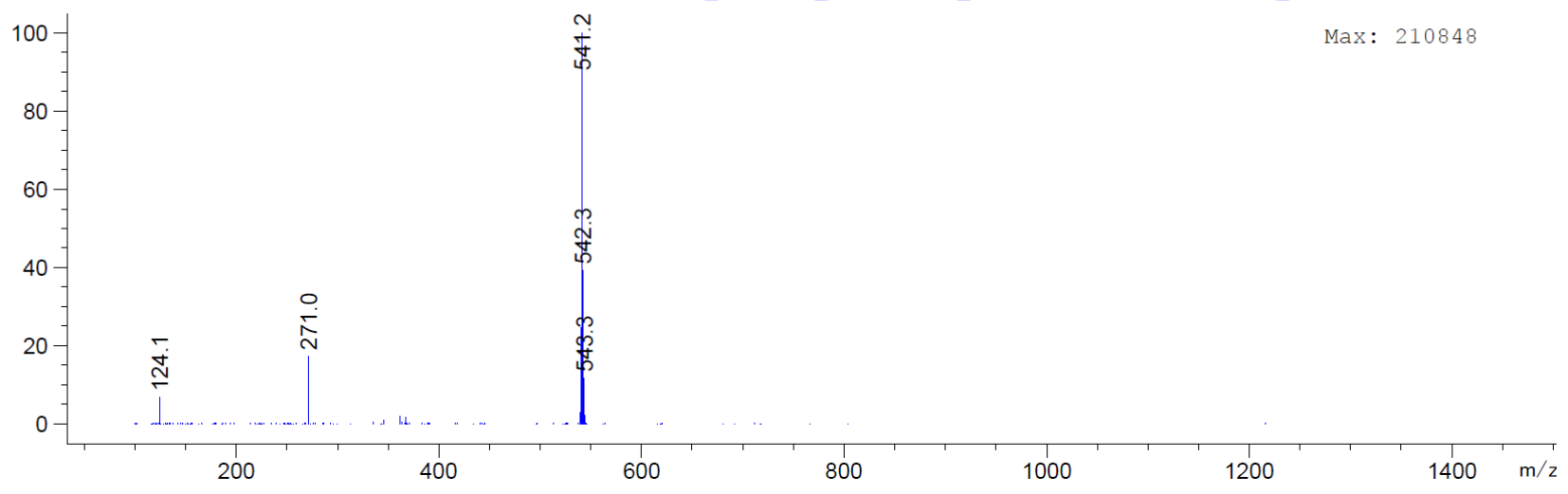



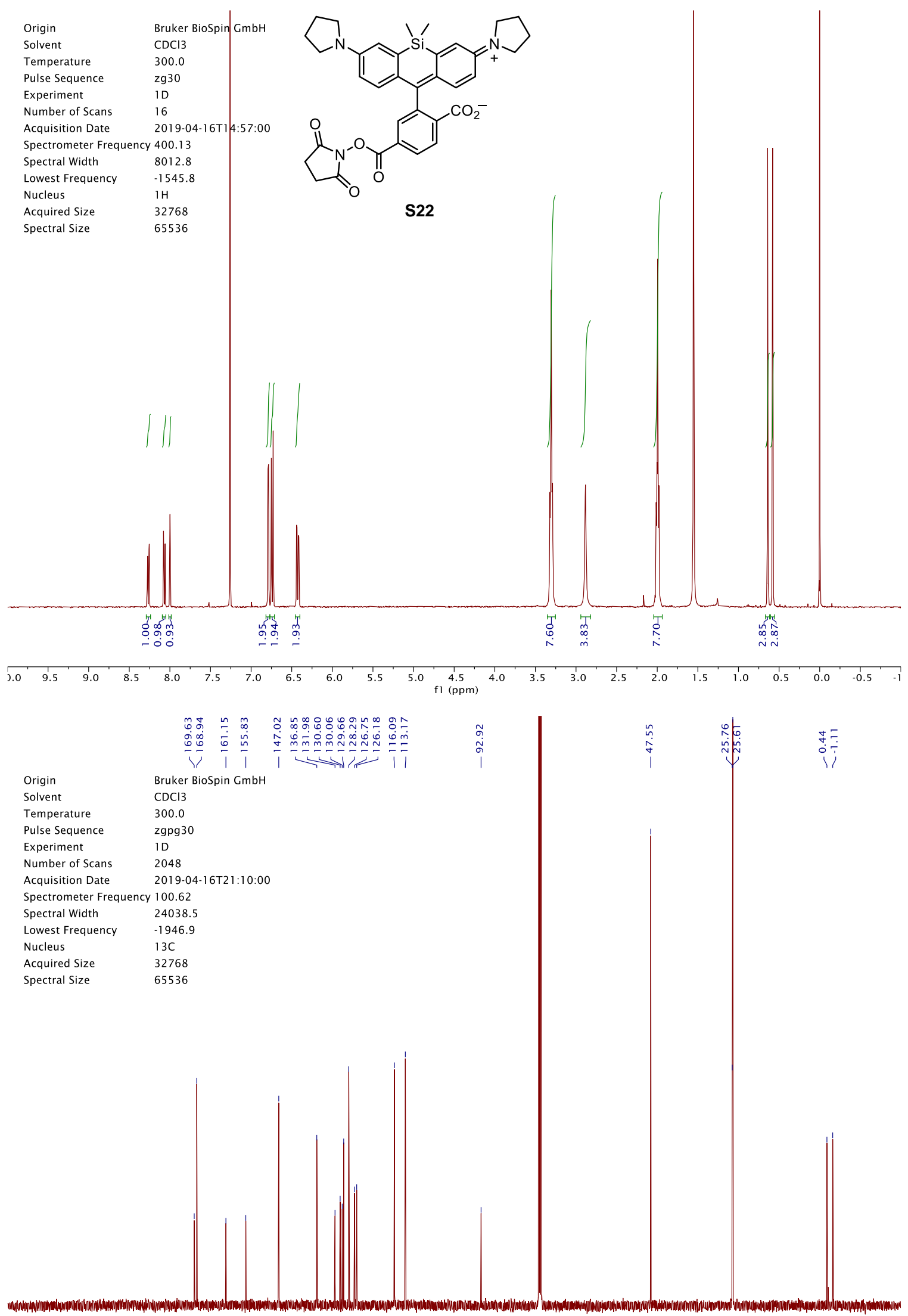

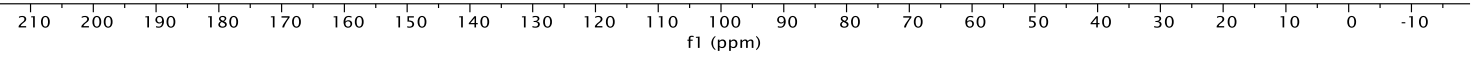




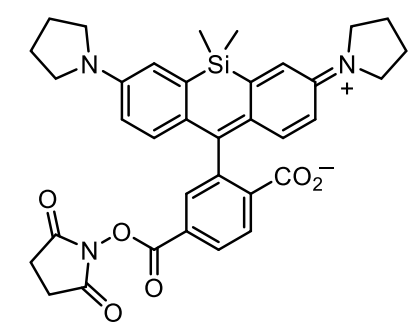

S22

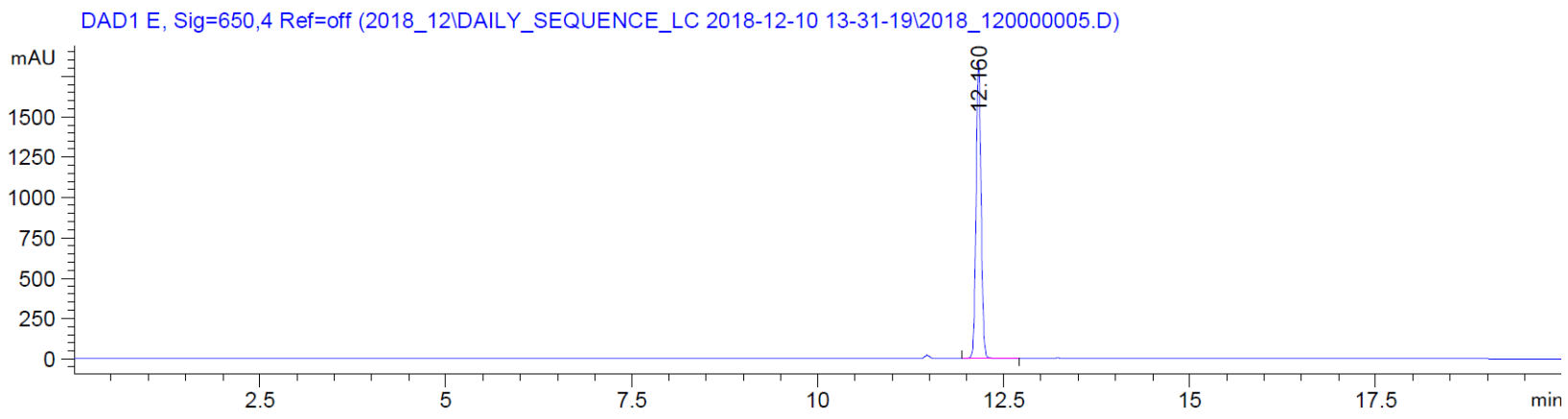

*MSD2 SPC, time=12.170:12.243 of C:ICHEM32I1IDATAI2018_12IDAILY_SEQUENCE_LC 2018-12-10 13-31-1912018_120000005.D ES-

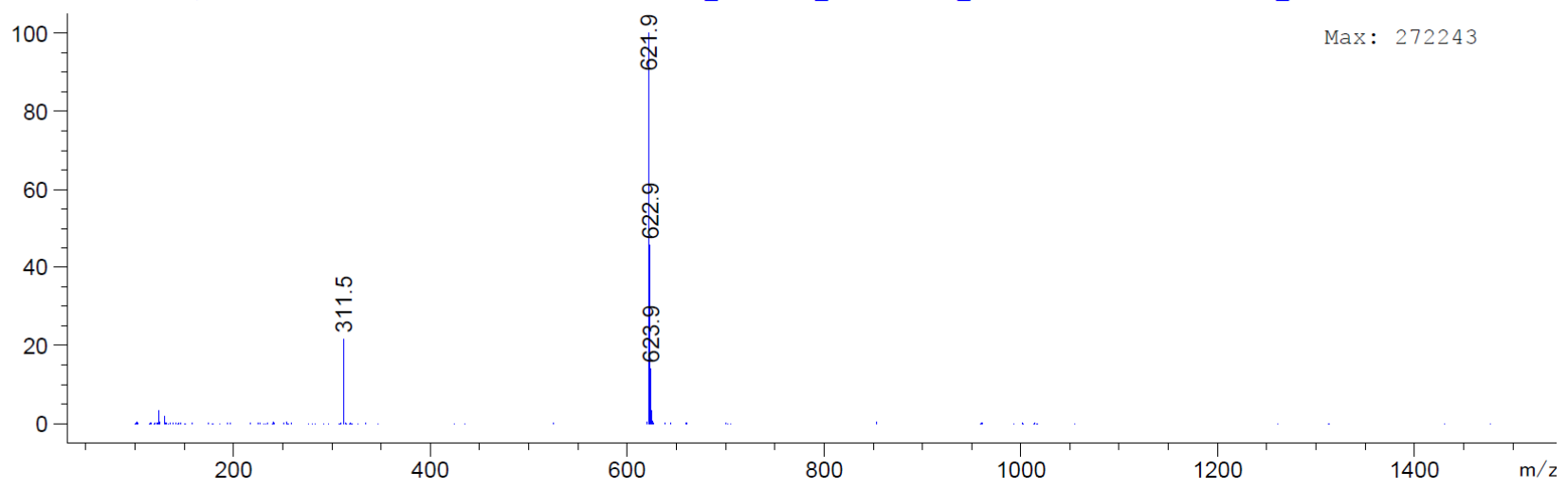




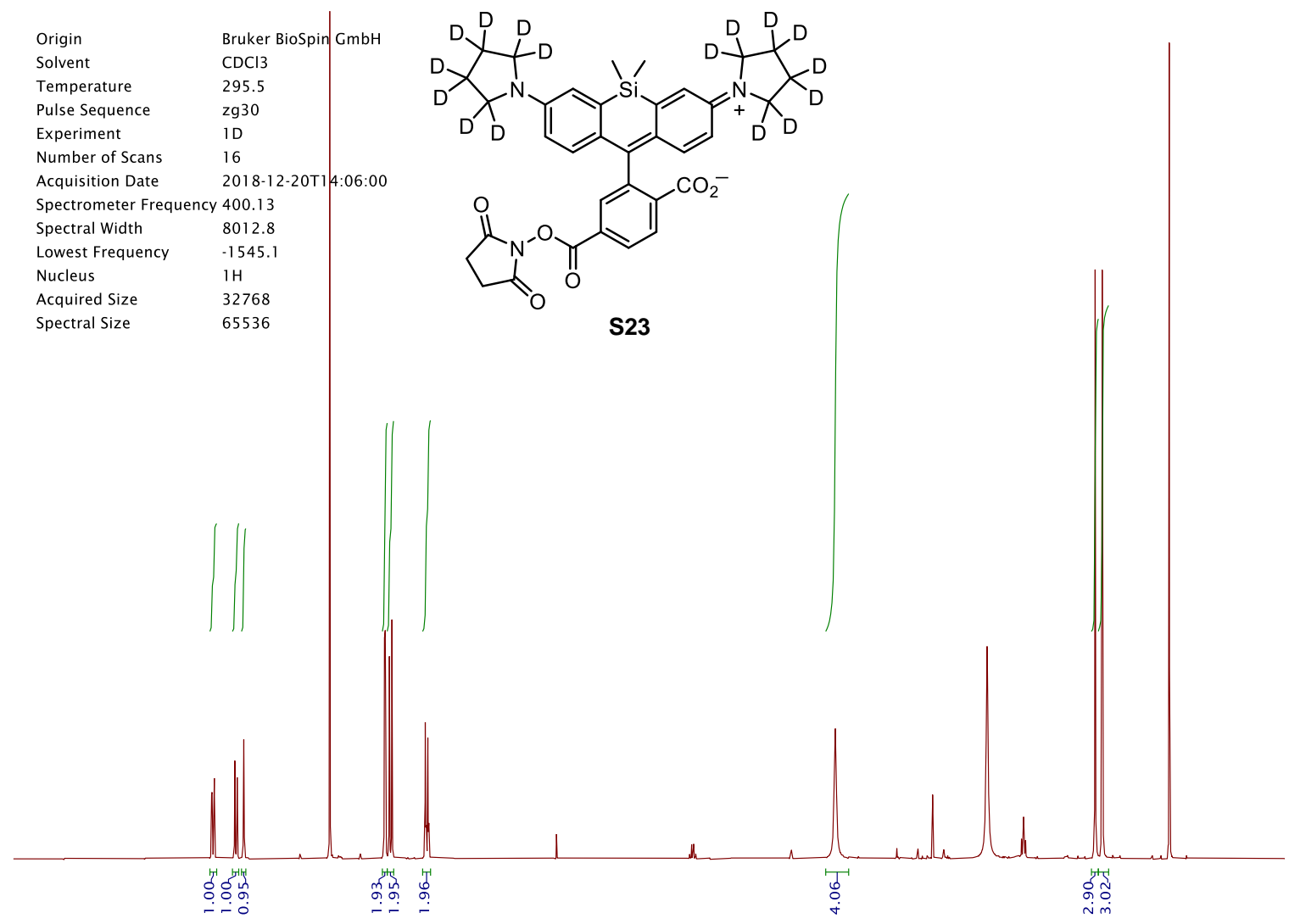

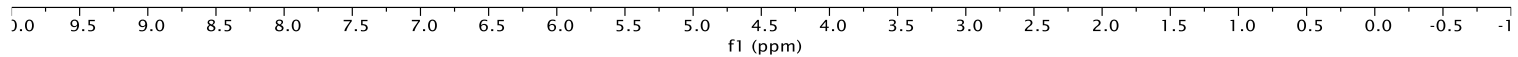

DAD1 E, Sig=650,4 Ref=off (2018_12IDAILY_SEQUENCE_LC 2018-12-10 13-31-1912018_120000002.D)

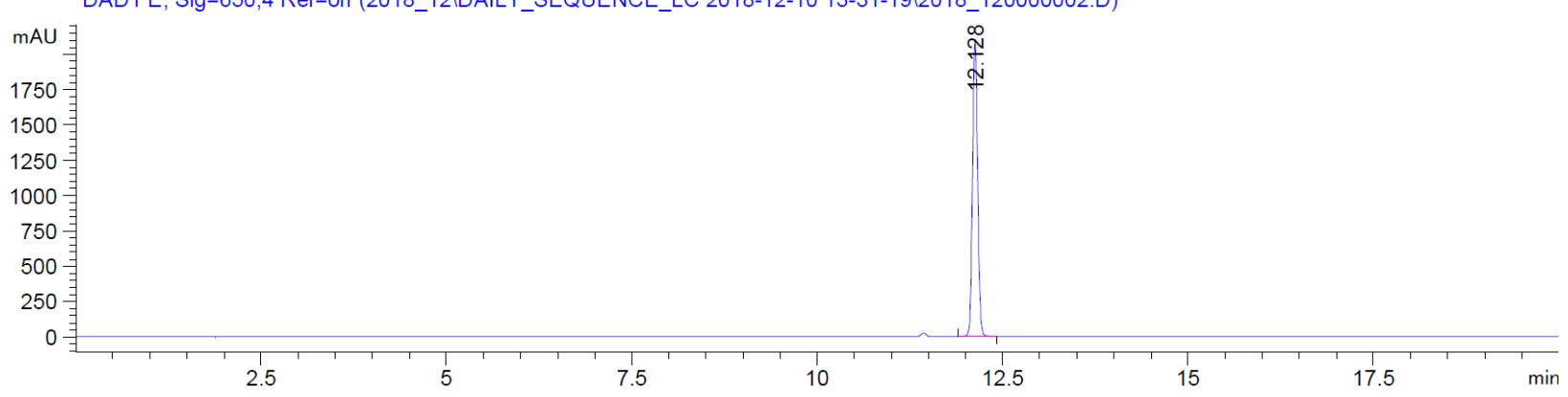

*MSD2 SPC, time=12.134:12.206 of C:ICHEM32I1IDATAI2018_12IDAILY_SEQUENCE_LC 2018-12-10 13-31-19l2018_120000002.D ES-

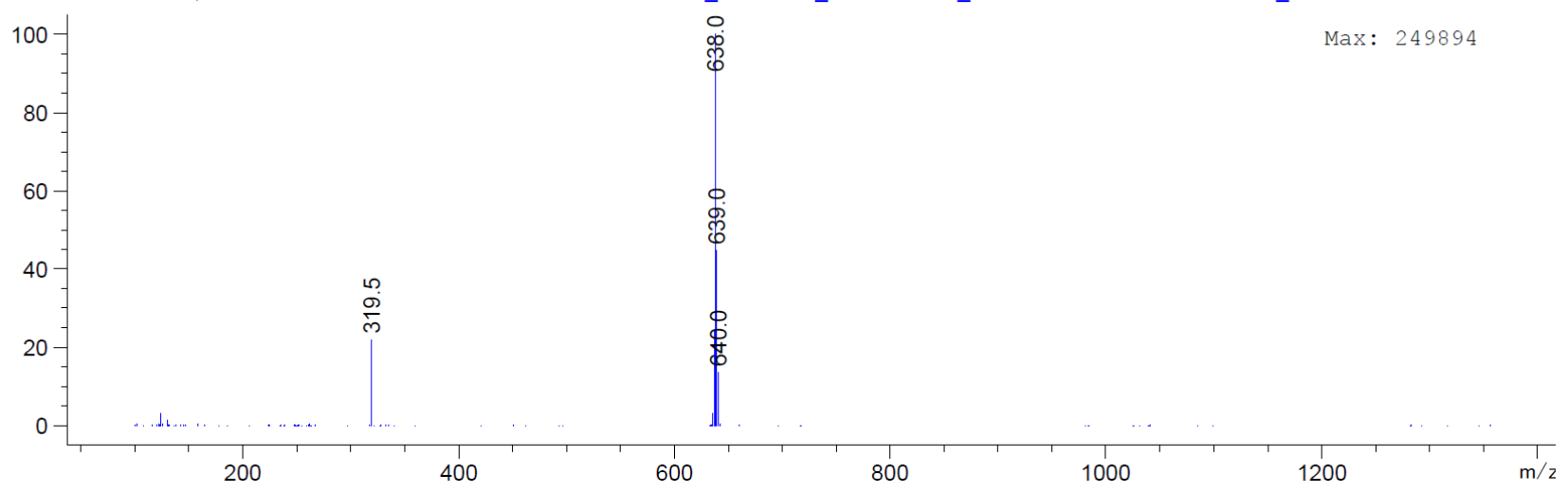




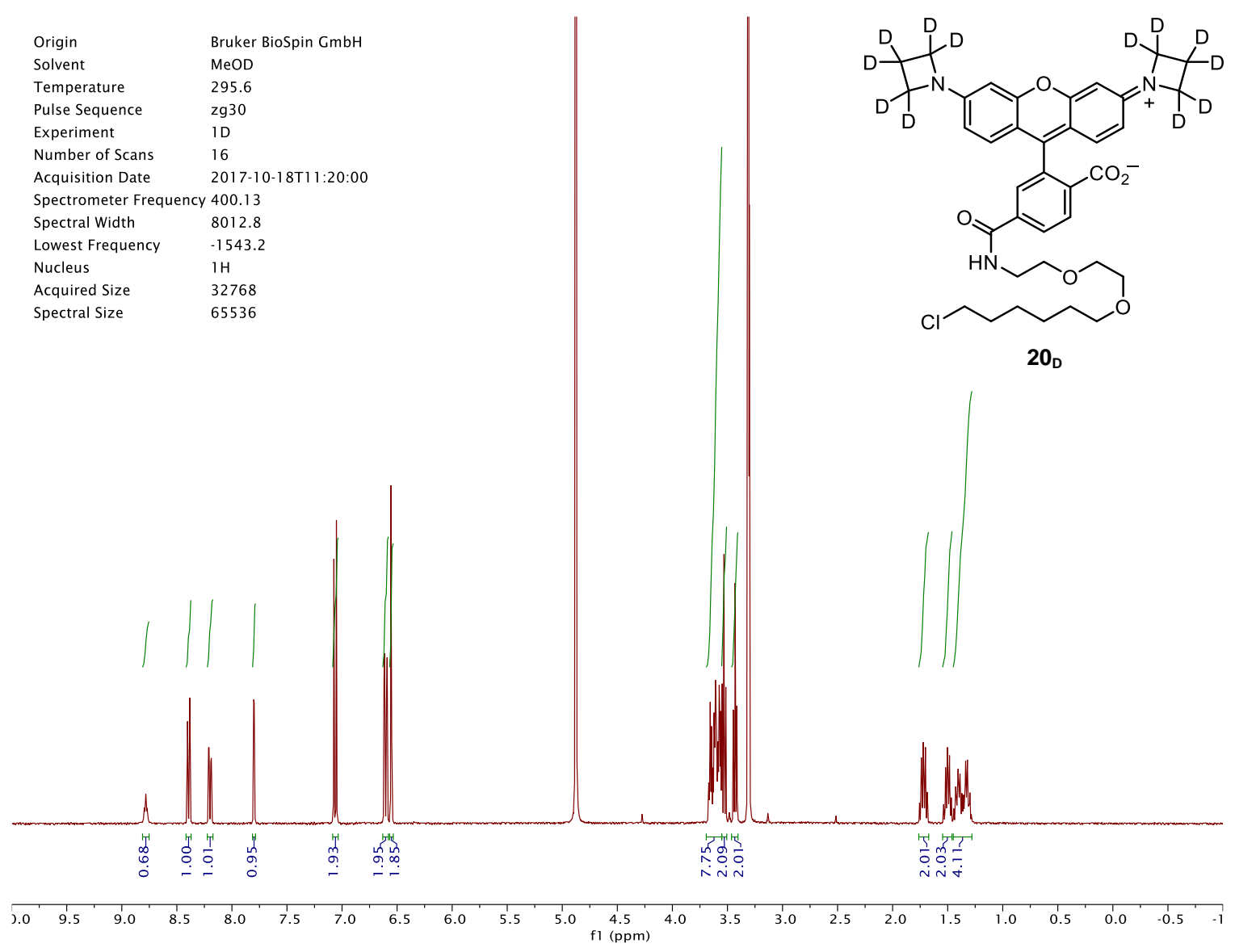

DAD1 C, Sig=550,4 Ref=off (2017_10IDAILY_SEQUENCE_LC 2017-10-17 15-38-37/2017_100000002.D)
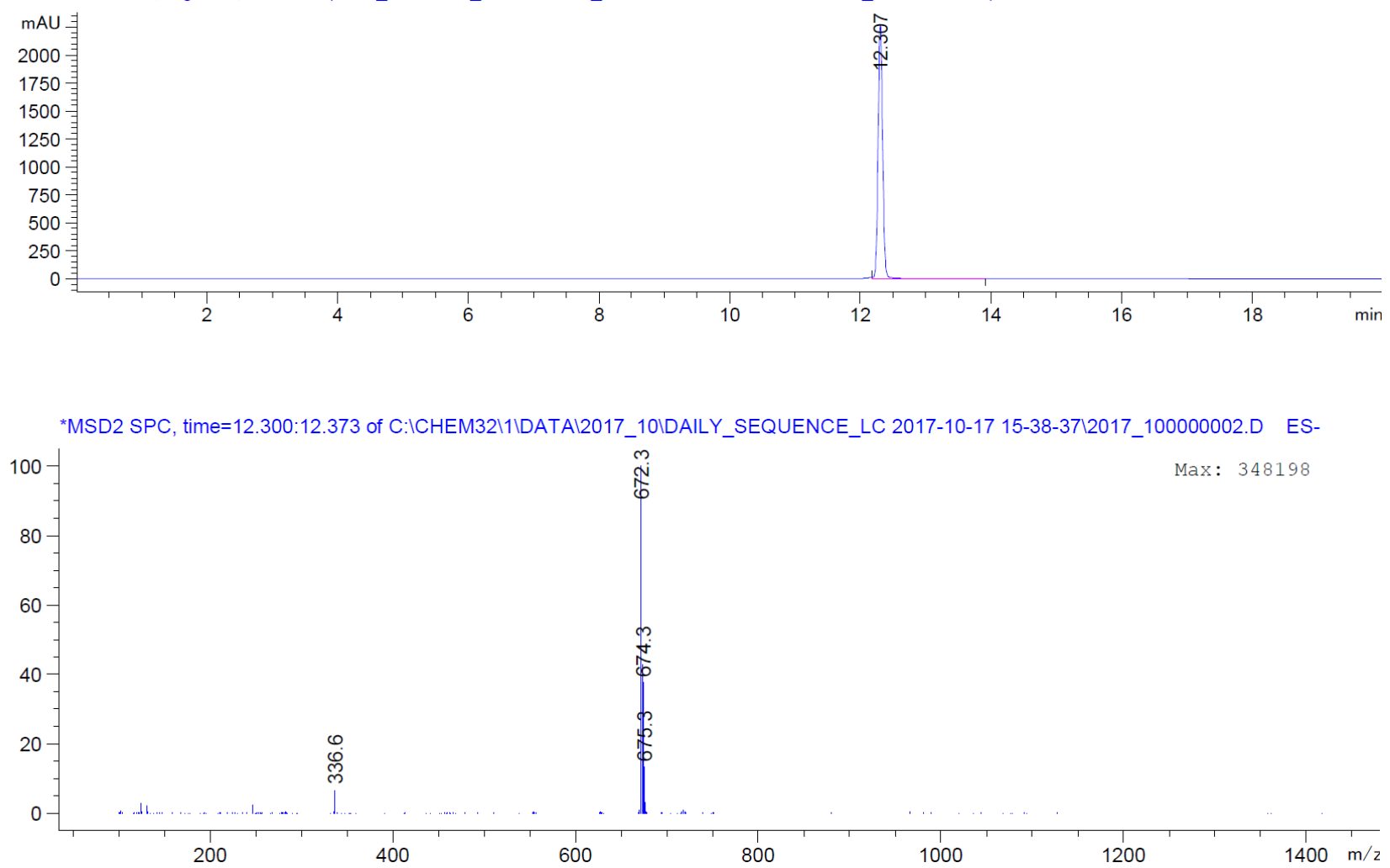


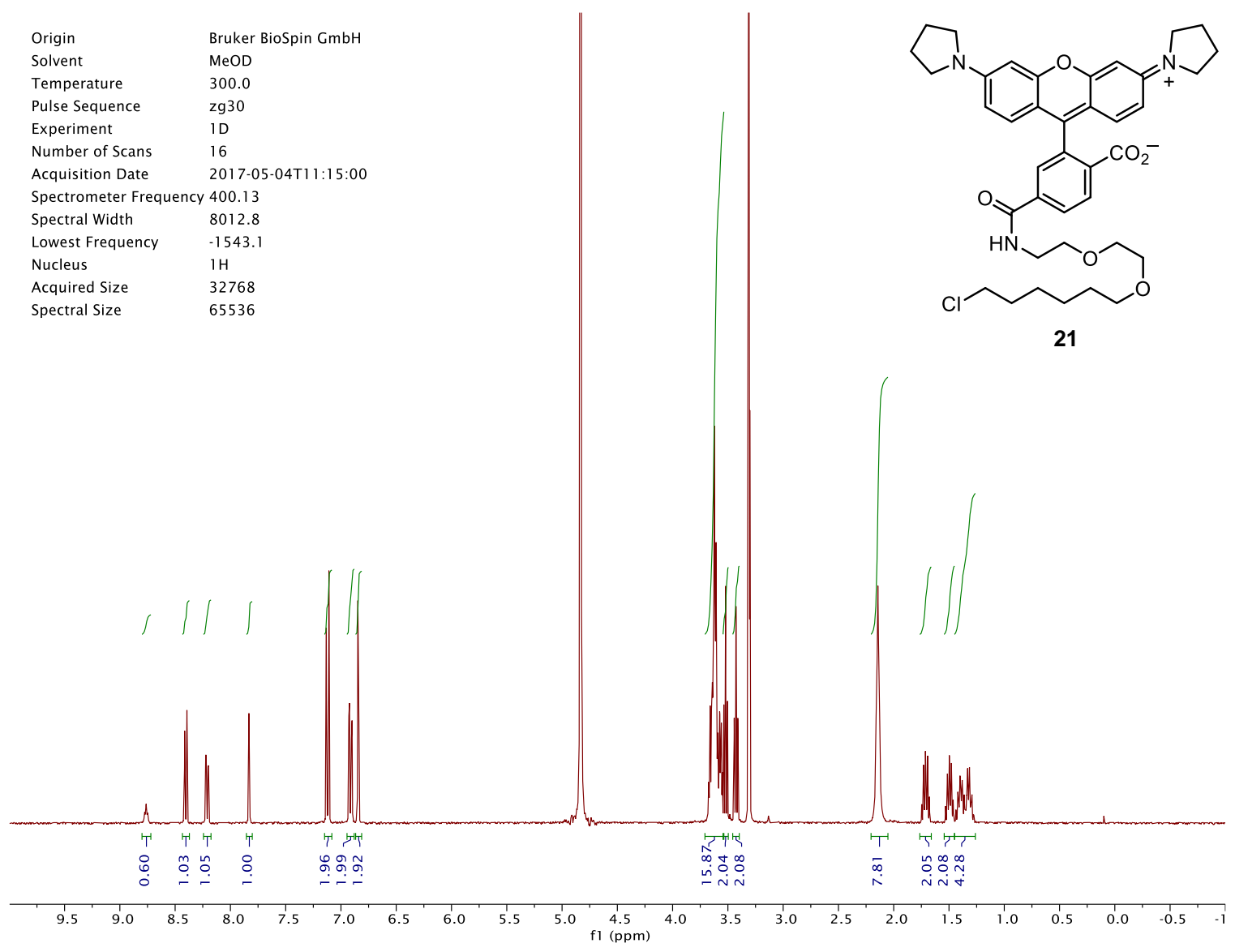

DAD1 C, Sig=550,4 Ref=off (2017_04IDAILY_SEQUENCE_LC 2017-05-02 11-11-3312017_040000003.D)
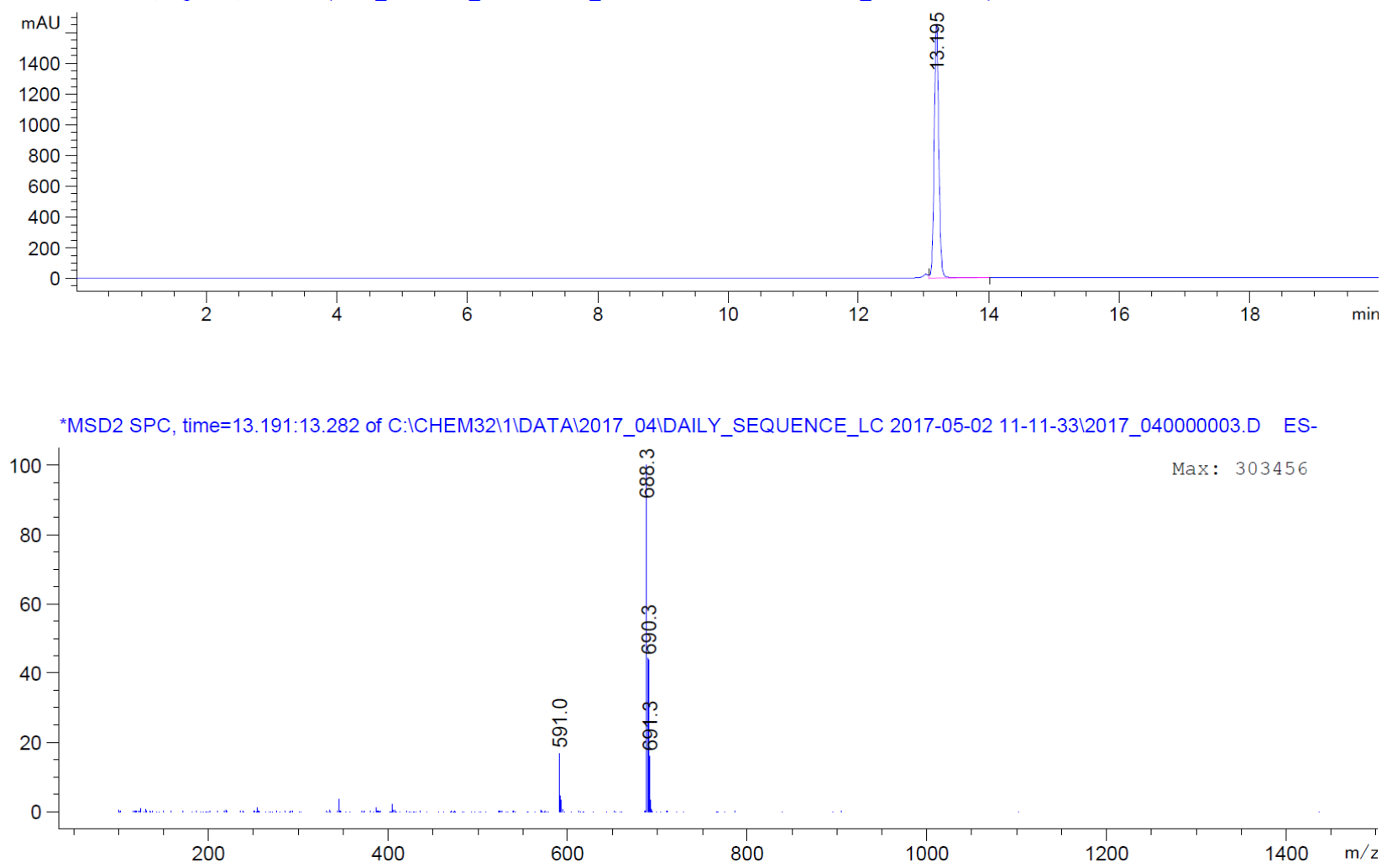


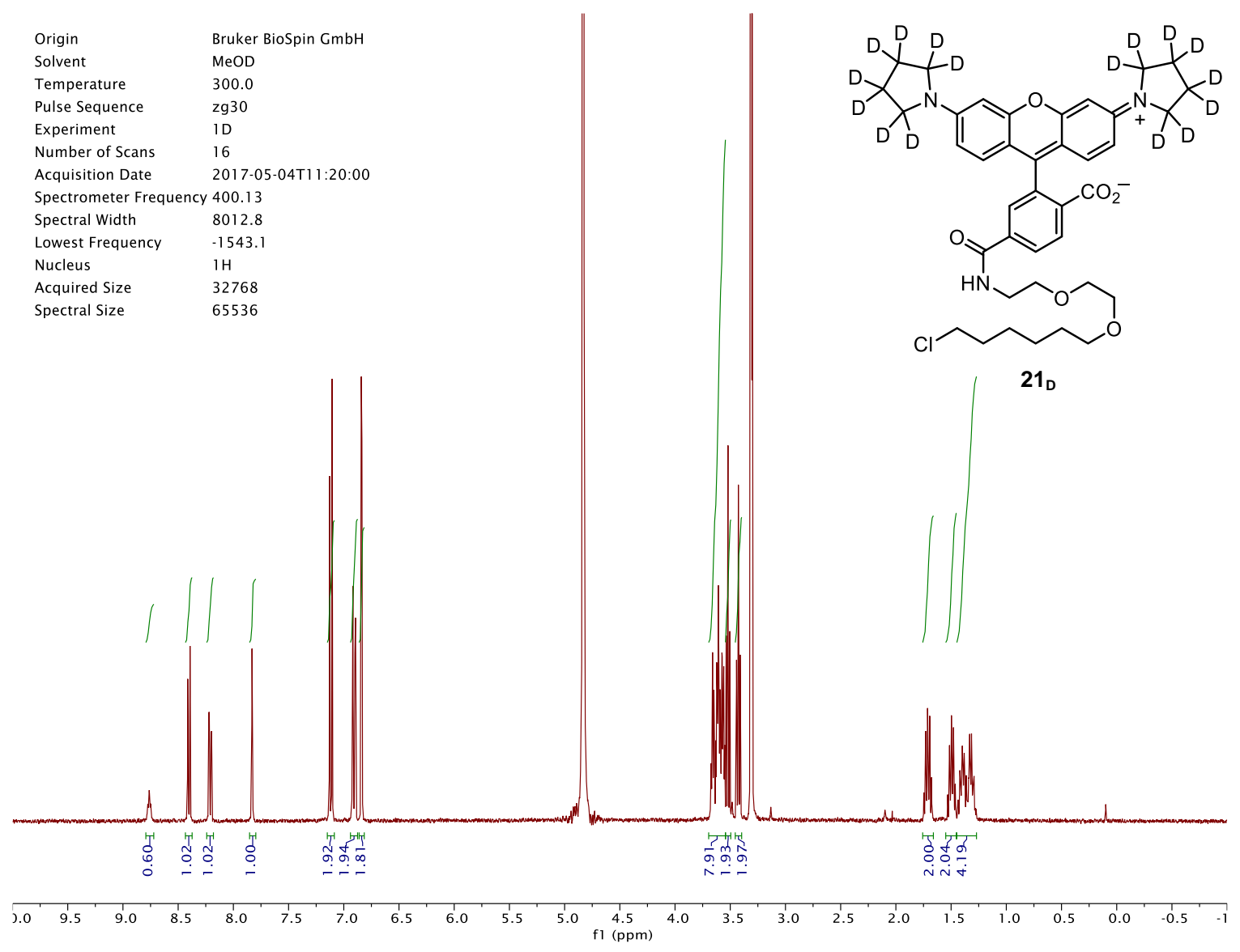

DAD1 C, Sig=550,4 Ref=off (2017_04IDAILY_SEQUENCE_LC 2017-05-02 13-05-0012017_040000002.D)

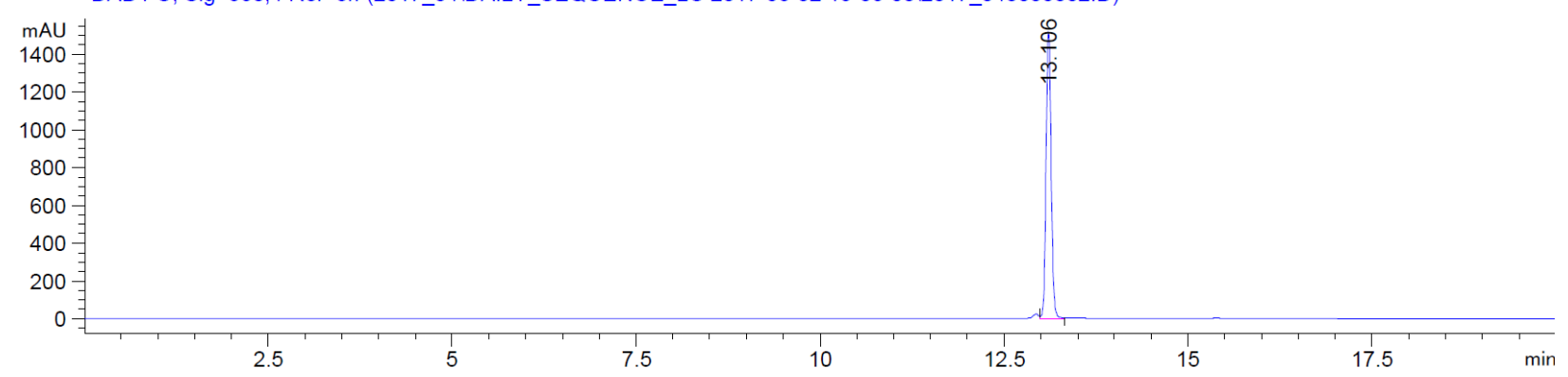

${ }^{*}$ MSD2 SPC, time=13.115:13.187 of C:ICHEM32I1IDATAl2017_04IDAILY_SEQUENCE_LC 2017-05-02 13-05-0012017_040000002.D ES-

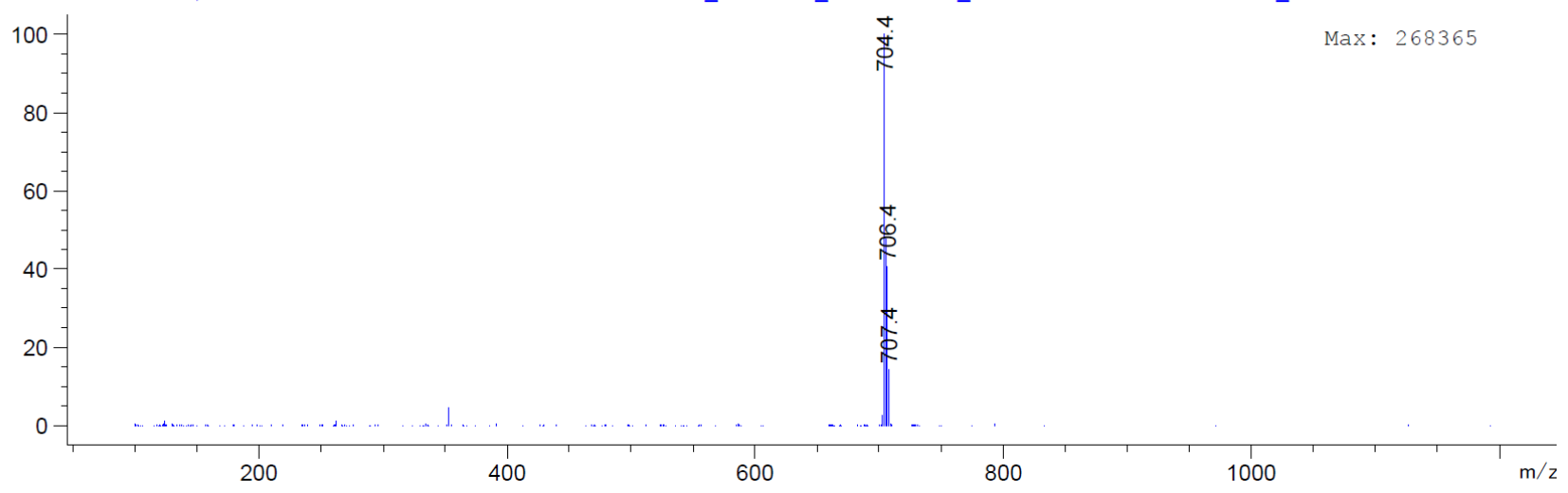




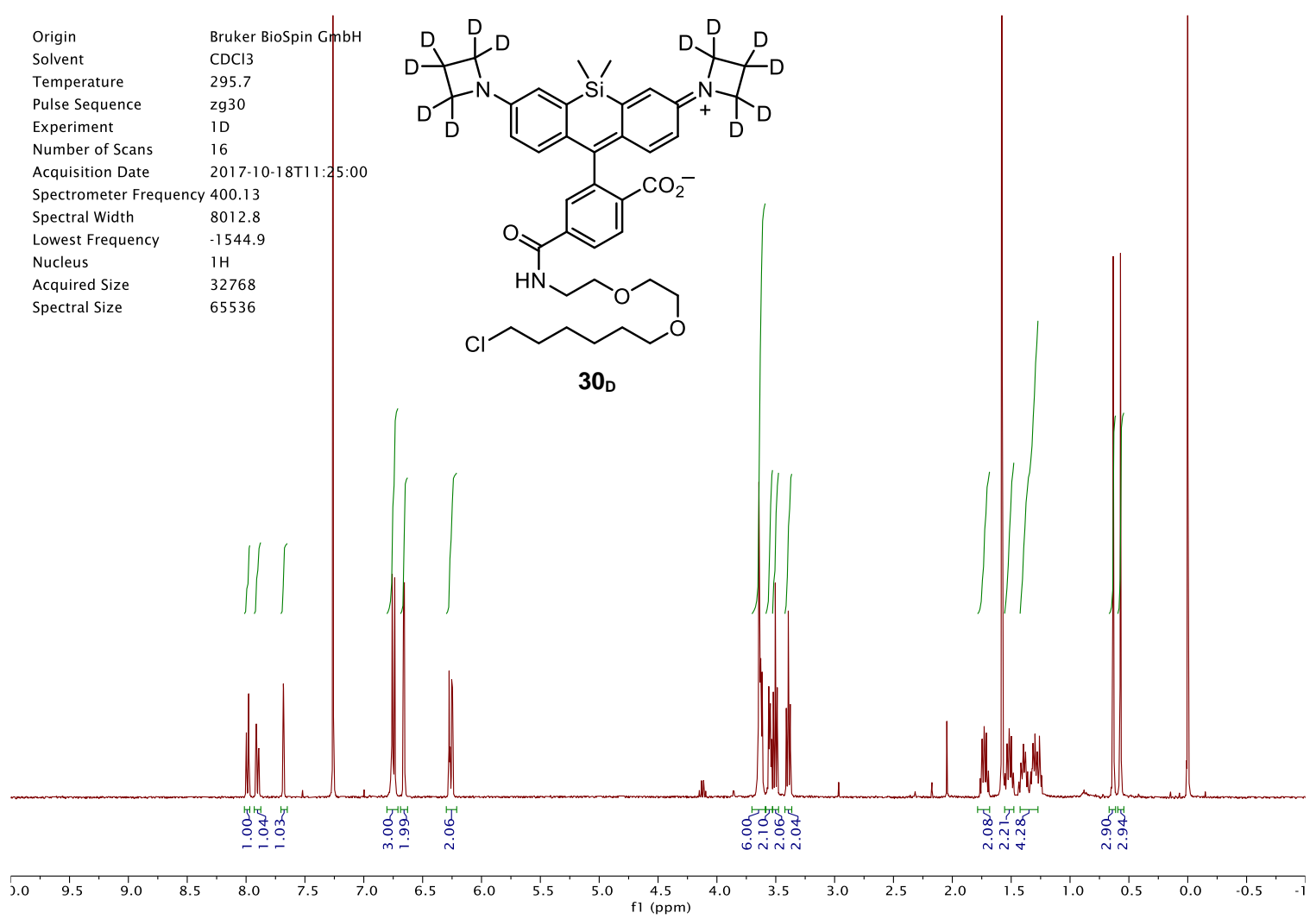

DAD1 E, Sig=650,4 Ref=off $\left(201712017 \_10 \mid\right.$ DAILY_SEQUENCE_LC 2017-10-18 12-18-1012017_100000003.D)

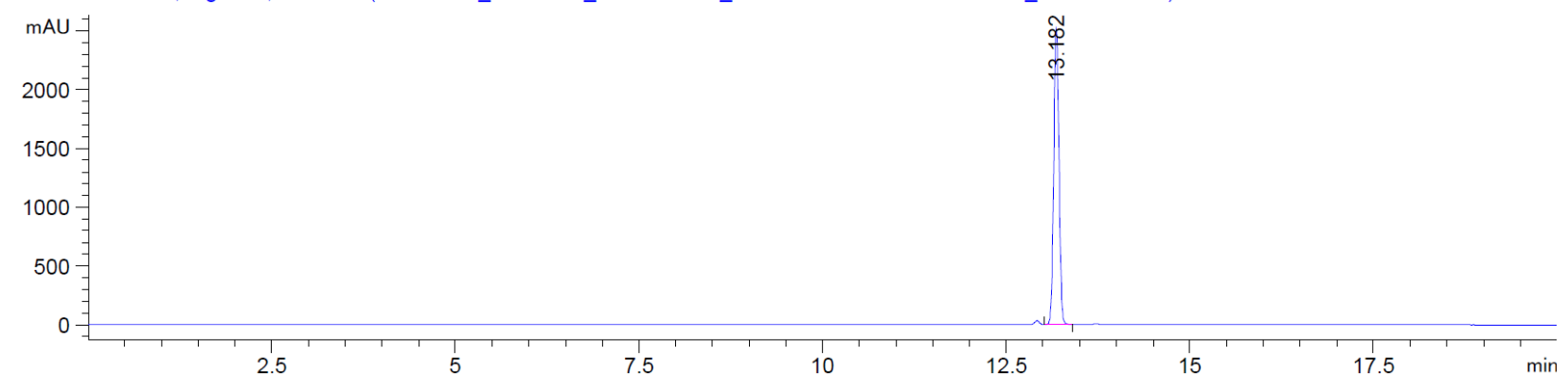

*MSD2 SPC, time=13.187:13.260 of C:ICHEM32I1IDATAI201712017_10IDAILY_SEQUENCE_LC 2017-10-18 12-18-1012017_100000003.D

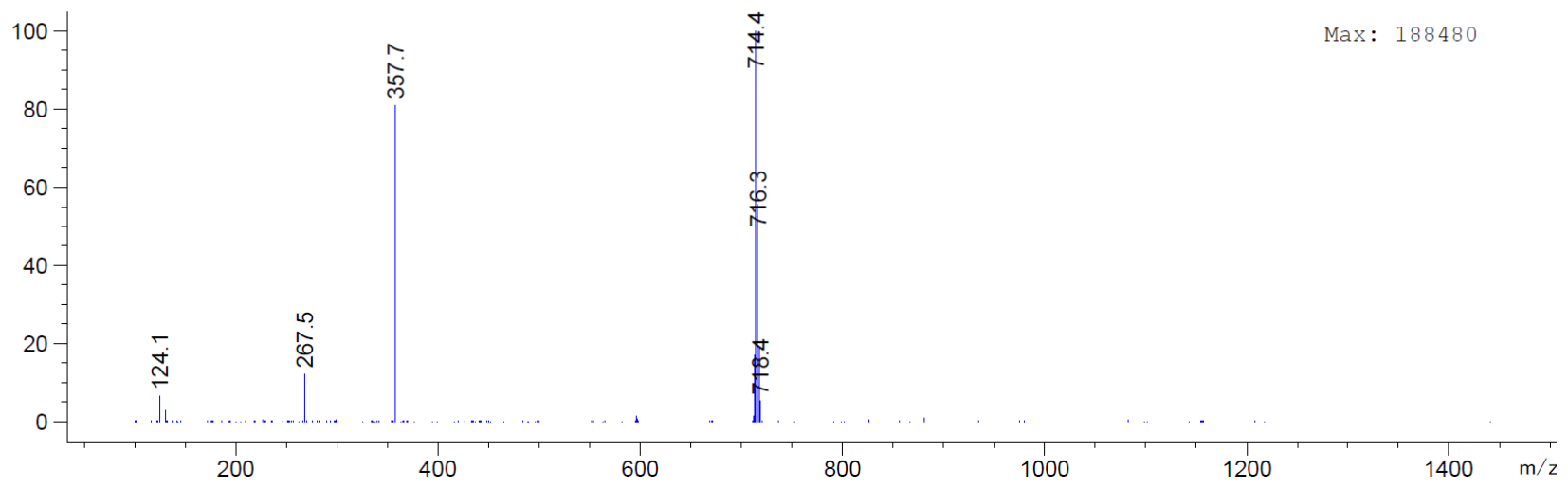




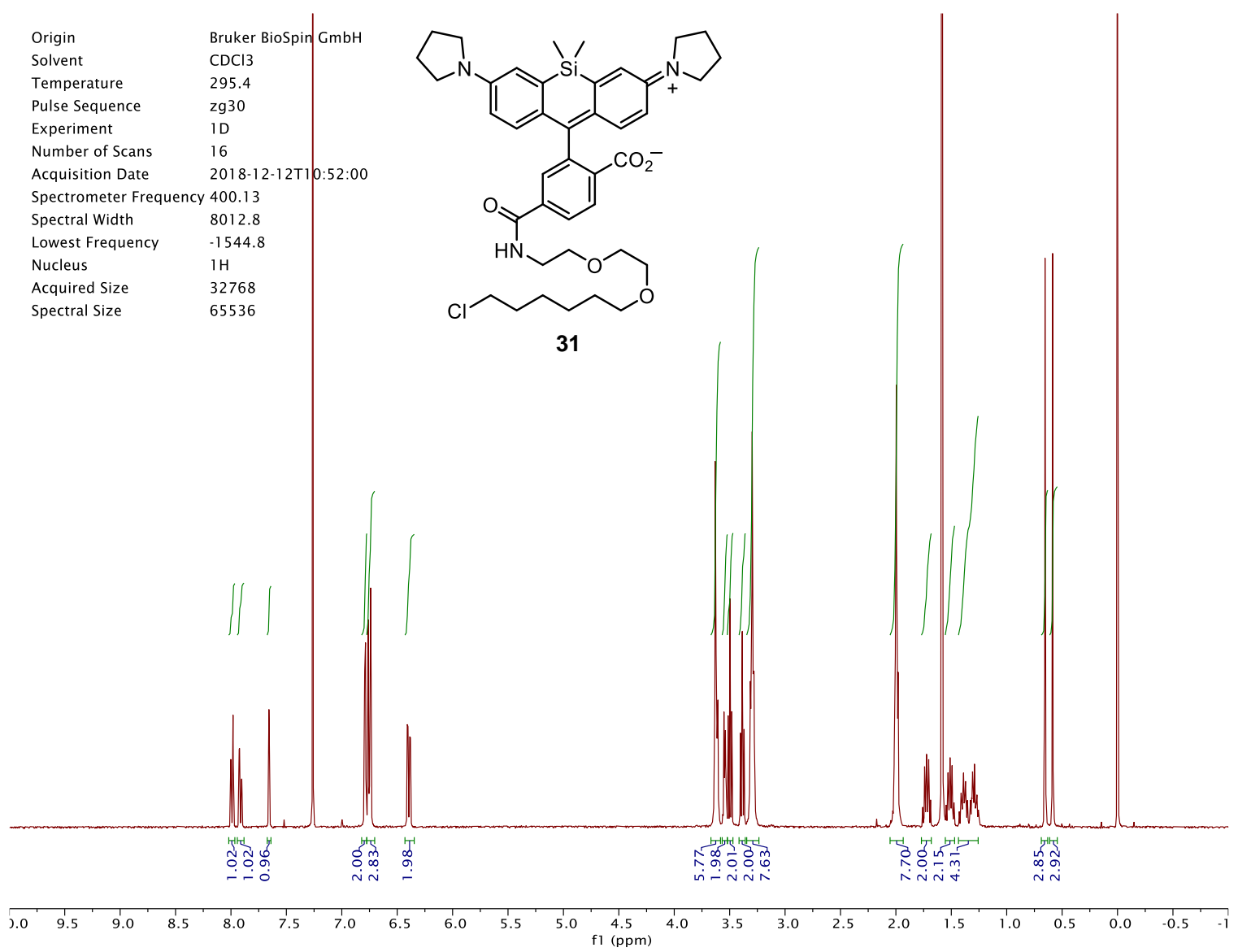

DAD1 E, Sig=650,4 Ref=off $\left(2018 \_12\right.$ IDAILY_SEQUENCE_LC 2018-12-11 16-14-0412018_120000002.D)

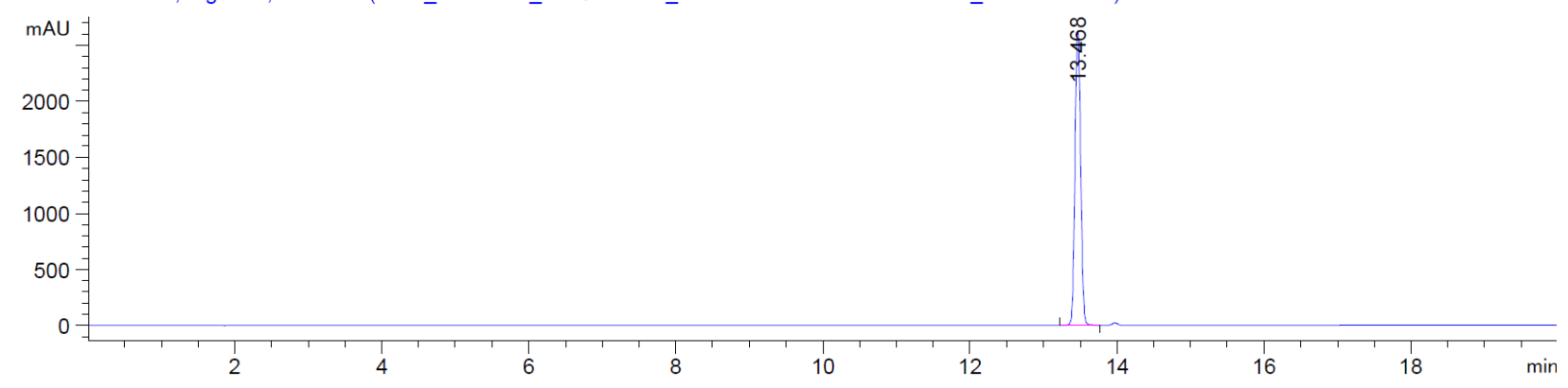

*MSD2 SPC, time=13.478:13.551 of C:ICHEM32I1IDATAI2018_12IDAILY_SEQUENCE_LC 2018-12-11 16-14-04l2018_120000002.D ES-

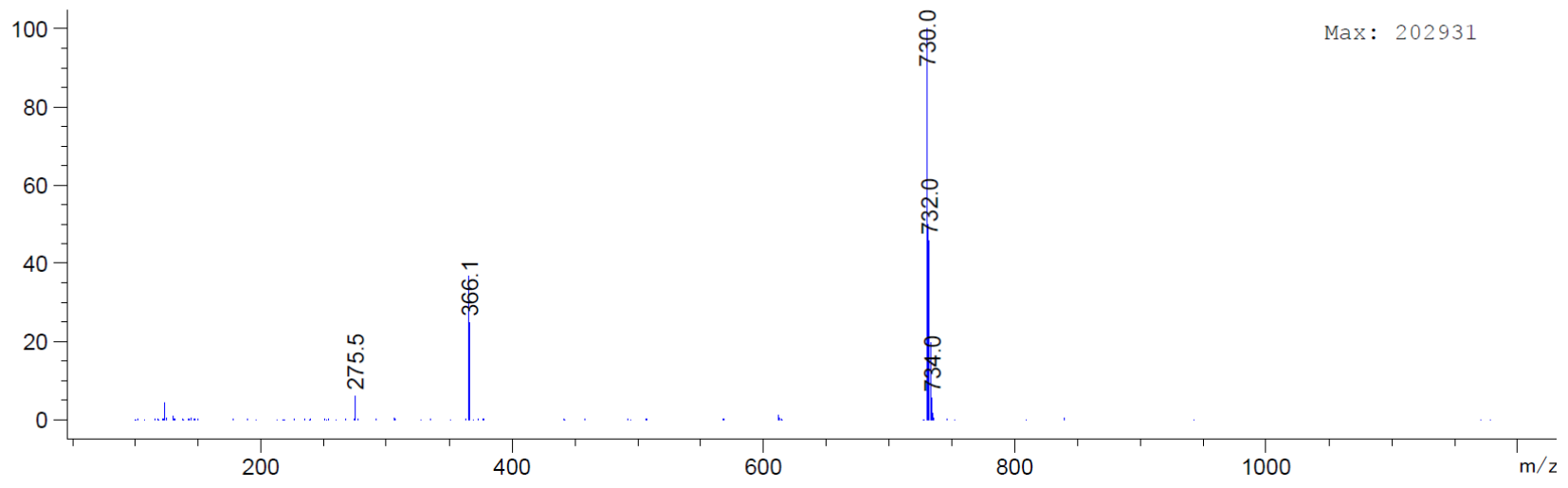




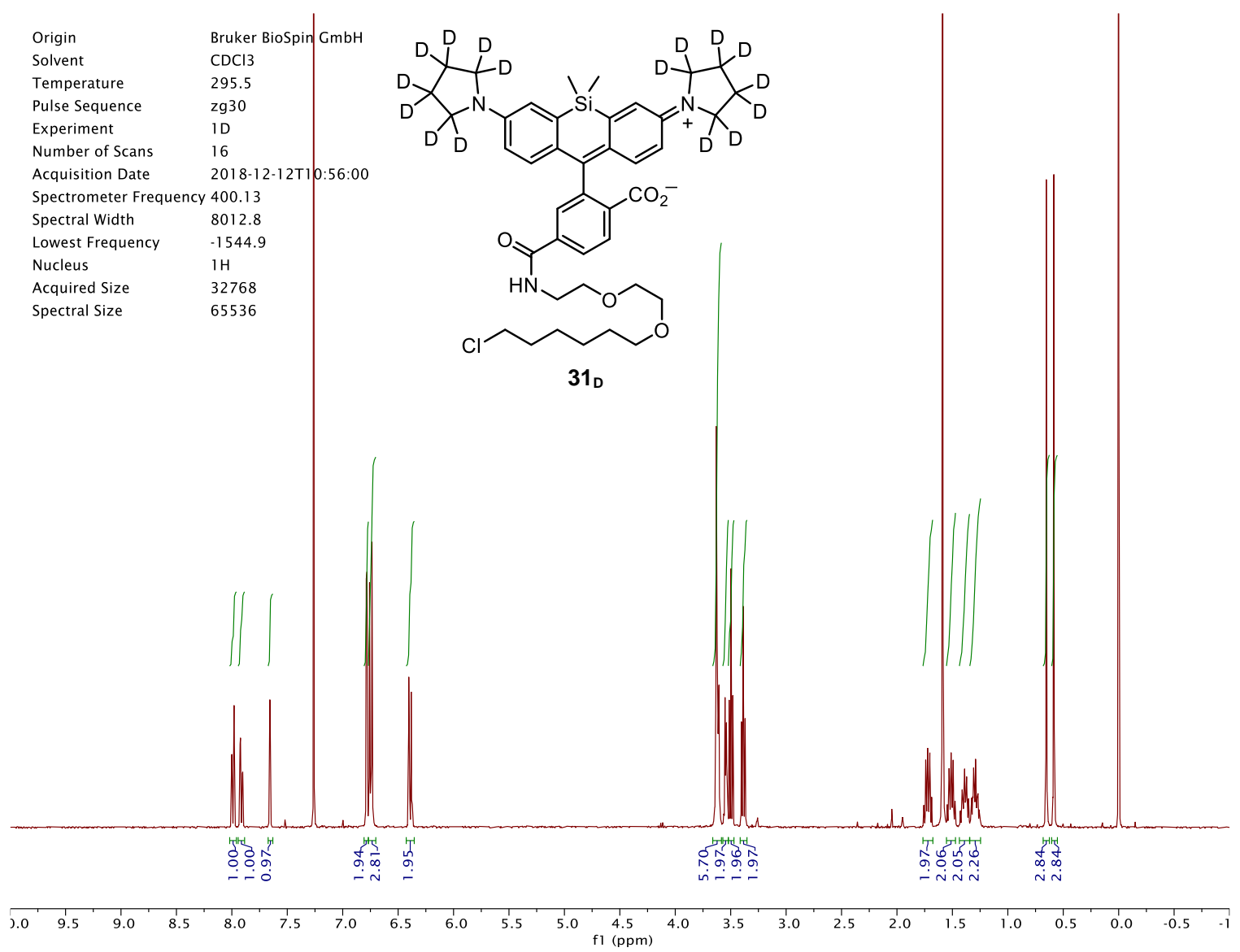

DAD1 E, Sig=650,4 Ref=off (2018_12IDAILY_SEQUENCE_LC 2018-12-11 16-14-04I2018_120000003.D)

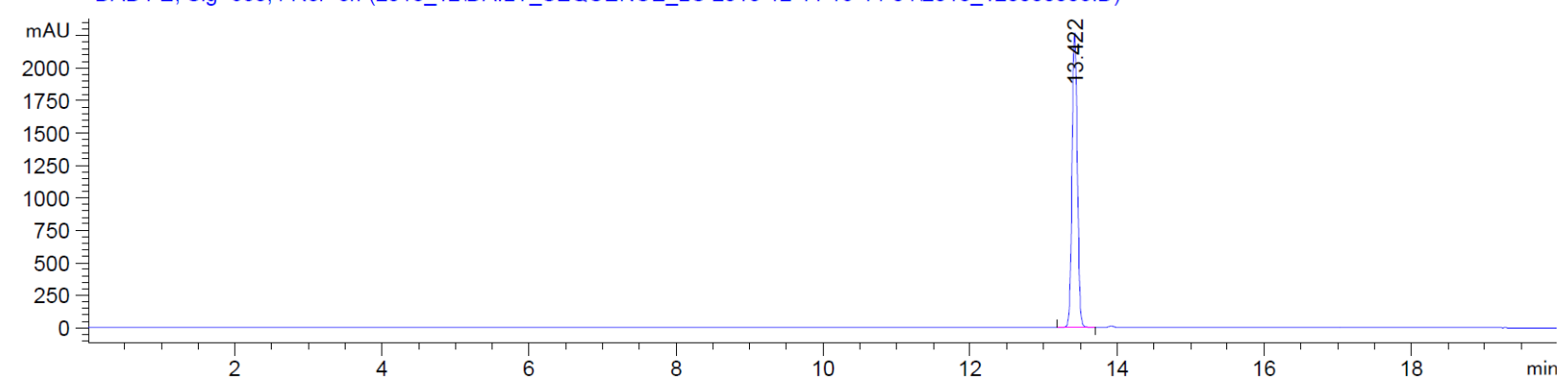

${ }^{*}$ MSD2 SPC, time=13.424:13.496 of C:ICHEM32I1IDATAI2018_12IDAILY_SEQUENCE_LC 2018-12-11 16-14-04l2018_120000003.D ES-

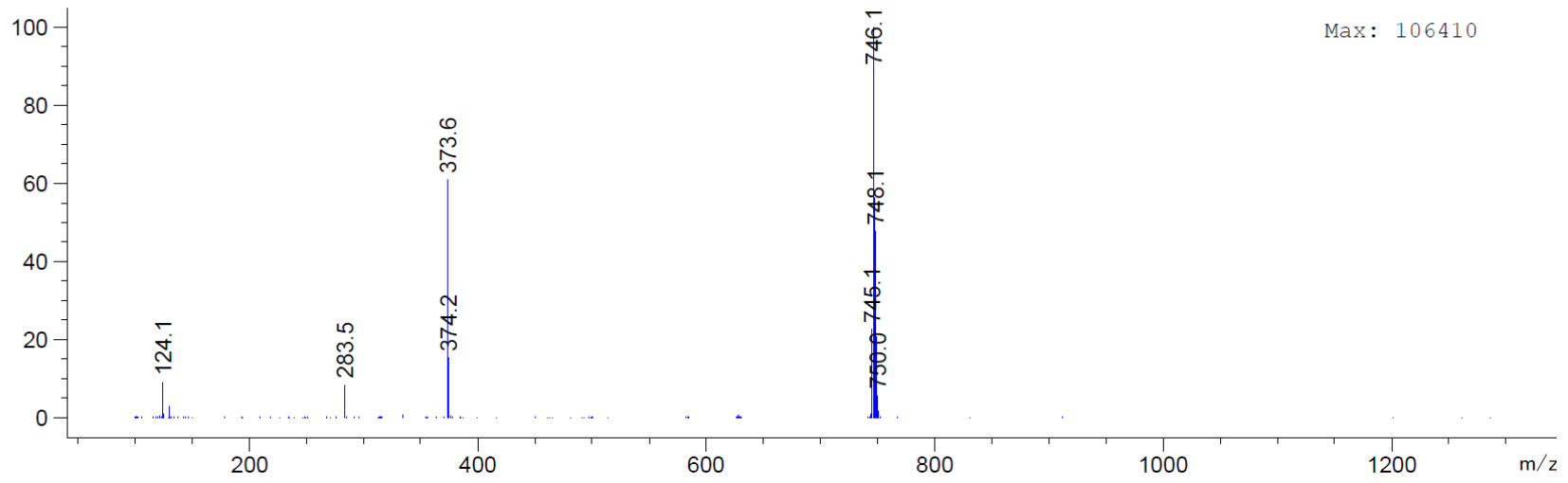

